\title{
Innovative training programs for frail elderly in the skilling up stage
}

Citation for published version (APA):

Rogan, S. (2016). Innovative training programs for frail elderly in the skilling up stage. [Doctoral Thesis, Maastricht University]. Datawyse / Universitaire Pers Maastricht. https://doi.org/10.26481/dis.20161215sr

Document status and date:

Published: 01/01/2016

DOI:

10.26481/dis.20161215sr

Document Version:

Publisher's PDF, also known as Version of record

\section{Please check the document version of this publication:}

- A submitted manuscript is the version of the article upon submission and before peer-review. There can be important differences between the submitted version and the official published version of record.

People interested in the research are advised to contact the author for the final version of the publication, or visit the DOI to the publisher's website.

- The final author version and the galley proof are versions of the publication after peer review.

- The final published version features the final layout of the paper including the volume, issue and page numbers.

Link to publication

\footnotetext{
General rights rights.

- You may freely distribute the URL identifying the publication in the public portal. please follow below link for the End User Agreement:

www.umlib.nl/taverne-license

Take down policy

If you believe that this document breaches copyright please contact us at:

repository@maastrichtuniversity.nl

providing details and we will investigate your claim.
}

Copyright and moral rights for the publications made accessible in the public portal are retained by the authors and/or other copyright owners and it is a condition of accessing publications that users recognise and abide by the legal requirements associated with these

- Users may download and print one copy of any publication from the public portal for the purpose of private study or research.

- You may not further distribute the material or use it for any profit-making activity or commercial gain

If the publication is distributed under the terms of Article $25 \mathrm{fa}$ of the Dutch Copyright Act, indicated by the "Taverne" license above, 


\section{INNOVATIVE TRAINING PROGRAMS FOR FRAIL ELDERLY IN THE SKILLING UP STAGE}

EFFECTS OF STOCHASTIC-WHOLE BODY VIBRATION AND DANCE VIDEO GAME ON PHYSICAL PERFORMANCE 
Copyright ${ }^{\odot}$ Slavko Rogan, Maastricht 2016 All rights reserved

Published by Datawys | Universitaire Pers Maastricht

ISBN 9789461596208 


\title{
INNOVATIVE TRAINING PROGRAMS FOR FRAIL ELDERLY IN THE SKILLING UP STAGE
}

\author{
Effects of stochastic-whole body vibration and \\ dance video game on physical performance \\ DISSERTATION
}

to obtain the degree of Doctor at the Maastricht University, on the authority of the Rector Magnificus, Prof. dr. L.L.G. Soete

In accordance with the decision of the Board of Deans,

to be defended in public on

Thursday 15th December 2016, at 12 hours

by

Slavko Rogan 


\section{Supervisor}

Prof. dr. R. A. de Bie, PhD

\section{Co-Supervisors}

PD. dr. E. D. de Bruin, PhD, Swiss Federal Institute of Technology Zurich, Switzerland

\section{Assessment Committee}

Prof. dr. H. Savelberg (chairman)

Prof. dr. J.M.G.A. Schols

Dr. K. Meijer

PD dr. med S. Bachmann (Kliniken Valens, Switzerland)

PD dr. med T. Hinrichs (Department of Sport, Exercise and Health, University of Basel, Basel, Switzerland)

The research presented in this thesis were conducted at the Faculty Health, Discipline Physiotherapy, of Bern University of Applied Sciences, Switzerland and at the School for Public Health and Primary Care: CAPHRI, Department of Epidemiology, of Maastricht University. CAPHRI participated in the Netherlands School of Primary Care Research CaRe. All studies were conducted at the Faculty Health, Discipline Physiotherapy, of Bern University of Applied Sciences, Switzerland and at the Canton Bern, Switzerland. 




\section{CONTENTS}

CHAPTER 2 Effects of whole-body vibration on postural control in elderly:

a systematic review and meta-analysis

CHAPTER 3 Effects of whole-body vibration on proxies of muscle strength in old adults a systematic review and meta-analysis on the role of physical capacity level

CHAPTER 4 "Skilling up" for training: a feasibility study investigating acute effects of stochastic resonance whole-body vibration on postural control of older adults

CHAPTER 5 Feasibility and effects of applying stochastic resonance whole-body vibration on untrained elderly: A randomized crossover pilot study

CHAPTER 6 Feasibility and informative data after stochastic resonance whole-body vibration intervention in long-term-care dwelling elderly: a pilot randomised double blinded cross-over study

CHAPTER 7 Sensory-motor training targeting motor dysfunction and muscle in long-term care elderly combined with motivational strategies: a single blind randomized controlled study

CHAPTER 8 Sensor-based foot-mounted wearable system and pressure sensitive gait analysis Agreement in frail older adults in long-term care

CHAPTER 9 General discussion 



\section{LIST OF ABBREVIATIONS}

\begin{tabular}{|c|c|c|}
\hline A & amplitude & STSML \\
\hline AP & anterior-posterior sway & ST \\
\hline $\mathrm{BMI}(\mathrm{Kg} / \mathrm{cm} 2)$ & Body Mass Index & STS \\
\hline CENTRAL & $\begin{array}{l}\text { Cochrane Central Register of } \\
\text { Controlled Trials }\end{array}$ & SR-WBV \\
\hline $\mathrm{Cm}$ & centimeter & SS-WBV \\
\hline CON & control group & \\
\hline CR (STS) & chair rising (Sit-to-Stand) & SV-WBV \\
\hline control & control group & TTS \\
\hline d & day & TUG \\
\hline DS & dynamic strength & VG \\
\hline DT & dual task & W \\
\hline ES & effect size & $\mathrm{W} / \mathrm{kg}$ \\
\hline ETGUG & expanded timed get up-and-go & w \\
\hline Ex & exercise group & WBV \\
\hline $\mathrm{F}$ & frequency of vibration platform & WHO \\
\hline FaME & Falls Management Exercise & WMD \\
\hline FRT & functional reach test & \\
\hline FRQ & frequency of training & \\
\hline FS & functional strength & \\
\hline Go-Go & independent person & \\
\hline $1^{2}$ & $\mathrm{I}^{2}$-statistic for heterogeneity & \\
\hline IMVC & $\begin{array}{l}\text { isometric maximum voluntary } \\
\text { contraction }\end{array}$ & \\
\hline IRFD & isometric rate of force development & \\
\hline $\mathrm{Hz}$ & Hertz & \\
\hline HLY & healthy life years & \\
\hline LTC & long-term care & \\
\hline $\mathrm{m}$ & meter & \\
\hline ML & medial-lateral sway & \\
\hline $\mathrm{mm}$ & millimeter & \\
\hline MMSE & Mini-Mental Status Examination & \\
\hline MoVo & Motivation-Volition program & \\
\hline $\mathrm{N}$ & Newton & \\
\hline $\mathrm{N} / \mathrm{s}$ & Newton/seconds & \\
\hline $\mathrm{Nm}$ & Newton-metre & \\
\hline $\mathrm{Nm} / \mathrm{kg}$ & Newton-meter/kilogram & \\
\hline $\mathrm{N} / \mathrm{ms}$ & Newton/milliseconds & \\
\hline No-Go & $\begin{array}{l}\text { person depending permanently on } \\
\text { assistance with severe functional } \\
\text { limitation }\end{array}$ & \\
\hline PA & physical activity & \\
\hline PEDro & Physiotherapy Evidence Database & \\
\hline PA & physical activity & \\
\hline POS & initial position & \\
\hline $\mathrm{RCT}$ & randomized controlled trial & \\
\hline RES & resistance training group & \\
\hline RFD & rate of force development & \\
\hline RTF & foot reaction time & \\
\hline RTH & hand reaction time & \\
\hline $\mathrm{S}$ & seconds & \\
\hline Sham & sham group & \\
\hline SLS & single-leg-stance & \\
\hline SMD & standardized mean differences & \\
\hline SOT & Sensory Organization Test & \\
\hline SPBB & Short Physical Performance Battery & \\
\hline Slow-Go & $\begin{array}{l}\text { depending on support in everyday } \\
\text { activities such as dressing, body care, } \\
\text { eating, using the toilet, } \\
\text { mobility and planning the day }\end{array}$ & \\
\hline
\end{tabular}

Semi-Tandem Stand medio-lateral single task

semi-tandem stand

stochastic resonance whole-body vibration

side-alternating sinusoidal whole-body vibration sinusoidal vertical whole-body vibration time to stabilization

Time-up-and-Go exergaming / video gaming watt watt/kilogram week whole-body vibration World Health Organization weighted mean difference 



\section{Chapter 1}

General introduction 


\section{Background}

Between now and 2050 the number of elderly individuals in industrialized countries will increase by more than $70 \%$ [1]. It has been postulated that the number of Swiss residents aged 80 years and above will increase from currently 290000 to 720000 by 2060 [2]. High age, however, is associated with increased multimorbidity [3] and eventually loss of independency in daily life. At the beginning of 2000 between $9.1 \%$ and $11.4 \%$ of Swiss elderly aged 65 and older were in need of care. The number of elderly and very elderly in need of care in Switzerland is increasing continuously and, depending on the population scenario, could rise to $36 \%$ by 2020 [4]. Hence, the Swiss healthcare system will face an increased need for care of the elderly that may result in huge healthcare costs, while care service tariffs for the elderly are three to five times higher compared to younger people [5].

\section{Life expectancy and healthy life years at age 65}

In the last hundred years average life expectancy at birth in Switzerland has increased from 47 to 82.8 years $[6,7]$. This is due to an improvement in medical care, greater access to healthcare, and healthier lifestyles. Life expectancy is the most frequently used assessment of length of life. However data on life expectancy for different ages give a better overview of changing function and mortality patterns within a population [7]. In 2012, the average life expectancy of 65 year olds in EU member states was 18.9 years [6]. Sooner or later, people who live longer will inevitably reach the limits of physical and cognitive performance, or develop diseases. One possibility to express health status of a population is the average of healthy life years (HLY). It sums the number of years spent free of long-term activity limitation and is equivalent to disability-free life expectancy [8]. The average of HLY in Europe in 2012 at the age of 65 was 8.5 years for men and 8.7 years for women [8] (Figure 1). 


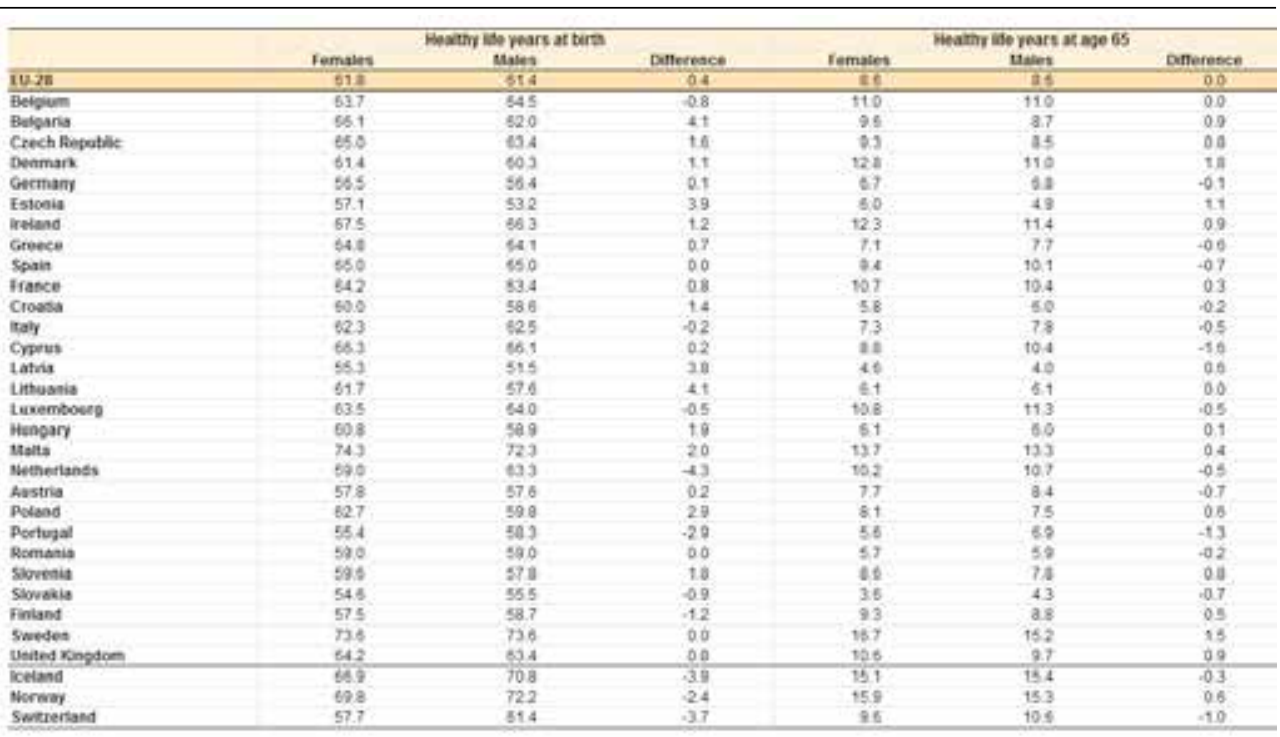

\section{Figure 1.}

Life expectancy and HLY at 65, by female, 2010 and 2014(Eurostat Statistics Databases, Joint Action: EHLEIS 2012)

The significant increase in life expectancy is associated with more need for long-term care (LTC). This involves relevant socio-political and economic public health Issues.

To tackle these efficiently, (public) health policy and decision makers require a more in-depth understanding of the demographic changes in society and they need better evidence-based knowledge of the phenomenon of aging. Finding ways and means to preserve health and physical and cognitive performance and to reduce (the impact of) multimorbidity and disability and their associated reduction in independency and loss of quality of life during the last years of life pose major scientific and public health challenges.

\section{Aging process}

Aging is a result of a combination of anatomical, biochemical, and physiological changes that occur over time $[9,10]$. Sooner or later, aging induces an increased risk of disability and dependency [11], modified by the fact whether individuals can live independently [12].

Aging is associated with a decline in muscle strength, muscle mass, balance, mobility and cognition influencing postural control and increased fall risk [13-19]. This loss of recourses is noticeable in everyday situations. In addition, sensimotor ability and cognitive function correlate negatively with increasing age [20].

The cognition permeation hypothesis describes how aging related reduced sensimotor function may lead to more dependency on cognitive resources [21]. Activities of daily living require attention and fast motor responses whilst constantly monitoring irrelevant or inappropriate inputs. 
In daily life, elderly individuals have more and more difficulties with coordinative processes when challenged in dual tasks [22].

Activities such as strength training, balance exercise or sensimotor training are methods with positive effects on muscle strength, balance and cognition, independent of age [23-26]. Therefore, it is essential to develop biological age- and performance-specific training programs and exercise concepts. The latter should be based on the latest scientifically proven evidence.

This thesis fits within the scope of these challenges because (i) it studies the impact of selected aging-related changes on determinants of function and independence in daily life, and (ii) investigates new training strategies to improve function and independency in elderly Swiss residents needing long-term care.

\section{Theoretical background of the innovative training program}

As mentioned above, physical activity and exercise play an important role $[18,27]$ in promoting health, improving function and extending independence in the elderly population. In addition, there is enough evidence that physical activity programs have positive effects on metabolic and cardiovascular disorders, and may reduce falls and the risk of osteoporosis, colon cancer, breast cancer, depression, and anxiety, as well as improving general well-being [28]. Suitable training and exercise programs for elderly individuals should include components from strength, endurance, balance, and agility [18, 29-48].

This thesis follows the Falls Management Exercise (FaME) program developed by Skelton and Dinan [49] designed to prevent falls in the elderly. The FaME program is divided into three phases. Phase 1 consists of "skilling-up" over the first 12 weeks, with the aim to improve neuromuscular function, technique and safety during exercise. Phase 2 refers to "training gain" during weeks 13 to 33 , with the goal to progressively improve neuromuscular adaption, lower and upper limb strength, postural alignment and gait technique. Phase 3 is about "maintaining the gains" from weeks 34 to 38 , to improve functional ability through progressive resistance and flexibility training, dynamic balance and sensory input, transition to free standing, and traveling. FaME does not recommend specific training methods and exercises. This thesis wants to pick up at this point and focuses on the skilling-up phase wherein the feasibility and effects of innovative training programs are investigated.

However, it should be noted that in elderly individuals biological age appears in different forms. Physical performance is a major determinant of functional independence for basic and instrumental activities of daily life such as eating or toileting or dressing [50-52]. Thus far, there is no regular physical performance and function classification for seniors, which evaluates the current state of the individual's physical performance and function level, with the goal of determining an individual and adapted form of exercise. 
For this reason, the classification of Zeyfang and Braun [53] was used. They described how physical and mental functions should be classified as an independent person (Go-Go), a needyperson with slight handicap (Slow-Go), and a person in need of care in the field of LTC with severe functional limitations (No-Go) such as prefrail and frail elderly, and elderly with mobility disability respectively (Fig. 2).

Go-Go and Slow-Go elderly individuals can exercise with traditional training regimes, in contrast to the No-Go elderly individuals because of their limited physical performance, among other reasons. Figure 2 illustrates the functional classification training concept.

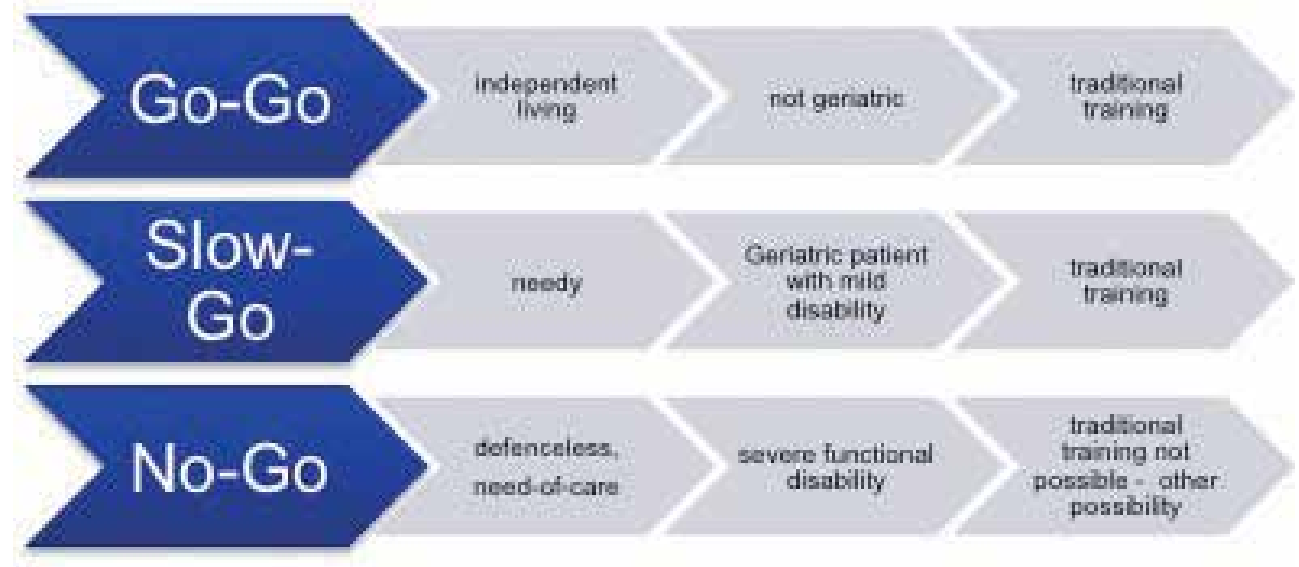

Fig. 2

Overview of the functional classification training program

A question that arises is what Is the feasibility of skilling-up methods for elderly in need for longterm care? Training regimes in the No-Go group (especially need of care elderly individuals with a mobility disability) should be designed in a way that they can be carried out within a short time frame. These training regimes should include specific exercise components of balance, strength and cognition. Furthermore, suitable assessments should be used to document the current functional status and the intervention (therapeutic) process.

Stochastic resonance whole-body vibration (SR-WBV) and exergaming / video gaming (VG) are suitable innovative training methods for skilling-up in elderly individuals with physical limitations such as elderly in need of care [54-57]. It can be assumed that the very small movements of the vibrating plates could be used as skilling-up for persons with mobility disability, offering a challenging form of balance and strength exercises. The literature indicates positive effects 
on balance and strength after WBV and VG training [58-75]. Elderly individuals improve their physical performance within a short time frame. Two types of WBV devices exist: sinusoidal WBV and stochastic resonance WBV (SR-WBV). At the physiological level, the neural system reacts differently to the two WBV types. There is some evidence that monotone (sinusoidal) vibration stimuli can lead to increased neuronal and muscular activity in the short term [76]. In the medium term, however, monotonic vibration may induce habituation and a concomitant reduction in neuronal activity $[77,78]$. The action potential of a nerve cell was generated earlier during stochastic compared to sinusoidal stimuli [77]. Because mechanical stochastic resonance producing small changes in strain on receptor membrane that leads to small fluctuation in receptor transmembrane potentials through changes in ion permeability [79]. The potential of the neuron is now closer to the threshold for firing an action potential in the presence of a weak stimulus.

Stochastic resonance WBV (SR-WBV) in particular seems to be suitable for No-Go elderly individuals, since the nervous systems ability to fully activate skeletal muscle voluntarily seems impaired in dynapenia, which might be activated through SR-WBV.

A systematic review [80] showed that cognitive elements should be a part of a training program for elderly individuals since they can enhance physical functions. One way to integrate cognitive elements into a training program is the use of VG because of its observed positive effects on cognitive processes $[64,66,81]$. VG combines dynamic functional movements, cognitive tasks and video gaming in order to enhance happiness and motivation. VG seems to have unique advantages compared to traditional therapy [82]. A benefit is that movement and behavior take place in a safe environment and can be shaped and graduated in accordance with the needs and level of ability of the participant engaging in treatment [82]. VG seems appropriate for skilling-up in the field of LTC for elderly in need of care.

Functional status screening may identify elderly individuals who need support. Physical performance assessments show potential as predictors for risk of clinical relevant disorders [50, 83, 84]. Therefore, the status of physical performance in No-Go elderly individuals should be determined. In this thesis the Short Physical Performance Battery (SPPB) by Guralnick et al. [83] and Guralnick and Winigrad [85] was applied. This Test Battery has been used as a prediction tool for possible mobility disability, chronic conditions, socioeconomic position and self-rated health [86] and may support the monitoring of functional performance in elderly individuals. The SPPB sums the number of points on the balance test, chair rise test and gait speed test. An SPPB score between 0 and 6 indicates "Poor Performance"; scores between 7 and 9 refers to "Intermediate Performance while values between 10 and 12 represents "High Performance". The literature shows that physical performance measures can be used to estimate the risk of hospitalization and a decline in health and function in clinical populations of elderly individuals [52]. This thesis focuses in persons who are in LTC. 


\section{Validity study}

In the last few years, accelerometer-based gait analysis systems are used in clinical daily routine. A mobile gait analysis system RehaWatch (HASOMED, Magdeburg, Germany) has been identified as a valid and reliable measure to detect differences in spatio-temporal gait parameters in young adults $[87,88]$. However, elderly have a more conservative gait pattern characterized by reduced walking speed, step length and trunk acceleration. It is unclear, whether the application of this system in elderly with mobility disability is valid. Therefore, the aim was to determine the concurrent validity of the RehaWatch ${ }^{\circledR}$ system with the GAITRite ${ }^{\circledR}$ system for temporal- and spatial-based gait parameters during single and dual task conditions that were recorded for averaged and individual step data in LTC elderly in need of care.

\section{Aim and Objectives}

Given that no research projects have been carried out with No-Go elderly individuals, this Doctoral Thesis was conducted to advanced our knowledge regarding training regimes in No-Goes. The aims of this Doctoral Thesis were to gain knowledge about the exact skilling-up training and exercise modalities in No-Go elderly individuals and to develop evidence-based and appropriate skilling-up training regimes for those persons.

However, in contrast to VG, studies examining the effects after SR-WBV on postural control and strength in an elderly population are lacking. Therefore, this thesis will start with the description of studies that were conducted to evaluate separately the feasibility and safety of SR-WBV in an elderly population. Afterwards a study was implemented where the effects of SR-WBV and VG in the field of LTC for elderly in need of care were evaluated. Based on the specific aims, the following research questions have been formulated:

1) How may whole-body vibration training influence postural control and muscle strength in elderly people aged over 65? (Chapter 2 and 3)

2a) Is SR-WBV feasible and safe in Slow-Go elderly individuals?

2b) What are the immediate and long-term effects after SR-WBV on postural control in Slow-Go elderly individuals?? (Chapter 4 and 5)

3) Is SR-WBV feasible and safe for No-Go elderly?

3b) What are the long-term effects after SR-WBV on postural control in No-Go elderly individuals? (Chapter 6)

4) Can the combined SR-WBV and exergaming in innovative skilling-up program improve the status of intermediate performance in No-Go elderly individuals within eight weeks instead of twelve weeks? (Chapter 7) 
5) How valid is the RehaWatch system for examining spatio-temporal gait parameters? (Chapter 8)

\section{Outline of this thesis}

This research project started with two systematic reviews and meta-analyses, followed by two feasibility studies and finally an RCT that tested the effectiveness of an innovative eightweek training regime on physical performance in the field of LTC for elderly in need of care. In addition, a validity study of the RehaWatch ${ }^{\circledR}$ system was carried out. In Chapter two the systematic review and meta-analysis presents the current state of the effects of 15 controlled trials after whole-body vibration on balance in an elderly population. Chapter three presents a systematic review of the literature about the current evidence for SR-WBV training. The metaanalysis of 38 controlled trials determined the effects of SR-WBV against control and exercise on isometric maximum voluntary contraction, dynamic strength, power, rate of force development and functional strength. In Chapter four, a short-term study evaluated how feasible and how safe SR-WBV intervention in Slow-Go elderly was. In addition, immediate effects on postural control are determined. Chapter five described a long-term crossover study lasting a period of eight weeks in Slow-Go elderly individuals. This study examined feasibility, safety, and effects on postural control. Chapter six reports the feasibility, safety and effects after SR-WBV intervention on physical performance in No-Go elderly individuals. In Chapter seven the innovative training program as skilling-up on No-Go elderly is described. Chapter eight present a validity study. The validity study compared the RehaWatch ${ }^{\circledR}$ system with the GAITRite system.

Chapter nine will discuss the main results and the resulting theoretical and methodological considerations of the previous chapters and will conclude with some clinical implications and future research in relation to skilling-up exercise on No-Go elderlies. 


\section{References}

1. Aging Nlo, Health Nlo: Global Health and Aging. In. Edited by Organization $\mathrm{WH}$; 2011.

2. Höpflinger F: Hochaltrigkeit demografische, gesundheitliche und soziale Entwicklungen. In., vol. 1. prosenectude.ch; 2003: 4-12.

3. Dwyer M, Gray A: Maintaining indepedency in old age: policy challenges. Social Policy Journal of New Zealand 1999, 13:83-94.

4. Höpflinger F, Hugentobler V: Pflegebedürftigkeit in der Schweiz. Prognosen und Szenarien für das 21. Jahrhundert. Bern, Switzerland: Hans Huber; 2003.

5. Casey $\mathrm{H}$, Oxley $\mathrm{H}$, Whitehouse $\mathrm{E}$, Antolin P, Duval R, Leibfritz W: Policies for an Ageing Society: Recent Measures and Areas for Further Reform. In.: OECD Publishing; 2003.

6. Union OE: Health at a Glance: Europe 2014: OECD Publishing.

7. Kinsella KG: Changes in life expectancy 1900-1990. Am J Clin Nutr 1992, 55(6 Suppl):1196S-1202S.

8. OECD: Health at a Glance:Europe 2014. In. : OECD Publishing; 2014.

9. Lord S, Sherrington C, Menz H, Close JC: Falls in older people risk factors and strategies for prevention, 2nd edn. Cambridge, GB: Cambridge University Press; 2007.

10. Oswald WD: [Can age and aging be measured?]. Z Gerontol Geriatr 2000, 33 Suppl 1:8-14.

11. Latham NK, Anderson CS, Lee A, Bennett DA, Moseley A, Cameron ID, Fitness Collaborative G: A randomized, controlled trial of quadriceps resistance exercise and vitamin $D$ in frail older people: the Frailty Interventions Trial in Elderly Subjects (FITNESS). J Am Geriatr Soc 2003, 51(3):291-299

12. Schaefer S, Schumacher V: The interplay between cognitive and motor functioning in healthy older adults: findings from dual-task studies and suggestions for intervention. Gerontology 2011, 57(3):239-246.

13. Rogan S, Radlinger L, Schmidtbleicher D, de Bie R, de Bruin E: The effects of wholebody vibration training on balance and gait in the elderly: a pilot study. In: 16th Annual Congress of the ECSS: 2011; Liverpool, GB. Liverpool: European College of Sport Science: $492-493$.

14. Tinetti ME, Speechley M: Prevention of falls among the elderly. N Engl J Med 1989, 320(16):1055-1059.

15. Tinetti ME, Speechley M, Ginter SF: Risk factors for falls among elderly persons living in the community. N Engl J Med 1988, 319(26):1701-1707.

16. Woollacott M, Shumway-Cook A: Attention and the control of posture and gait: a review of an emerging area of research. Gait Posture 2002, 16(1):1-14.

17. Woollacott $\mathrm{MH}$ : Systems contributing to balance disorders in older adults. J Gerontol A Biol Sci Med Sci 2000, 55(8):M424-428.

18. Aagaard P, Suetta C, Caserotti P, Magnusson SP, Kjaer M: Role of the nervous system in sarcopenia and muscle atrophy with aging: strength training as a countermeasure. Scand J Med Sci Sports 2010, 20(1):49-64.

19. Faulkner JA, Larkin LM, Claflin DR, Brooks SV: Age-related changes in the structure 
and function of skeletal muscles. Clin Exp Pharmacol Physiol 2007, 34(11):1091-1096.

20. Li KZ, Lindenberger U: Relations between aging sensory/sensimotor and cognitive functions. Neurosci Biobehav Rev 2002, 26(7):777-783.

21. Lindenberger $U$, Marsiske $M$, Baltes $P B$ : Memorizing while walking: increase in dualtask costs from young adulthood to old age. Psychol Aging 2000, 15(3):417-436.

22. Pizzigalli L, Filippini A, Ahmaidi S, Jullien $H$, Rainoldi $A$ : Prevention of falling risk in elderly people: the relevance of muscular strength and symmetry of lower limbs in postural stability. J Strength Cond Res 2011, 25(2):567-574.

23. El-Khoury F, Cassou B, Latouche A, Aegerter P, Charles MA, Dargent-Molina P: Effectiveness of two year balance training programme on prevention of fall induced injuries in at risk women aged 75-85 living in community: Ossebo randomised controlled trial. Bmj 2015, 351:h3830.

24. Shah $T$, Verdile $G$, Sohrabi $H$, Campbell A, Putland E, Cheetham C, Dhaliwal S, Weinborn $\mathrm{M}$, Maruff $\mathrm{P}$, Darby $\mathrm{D}$ et al: A combination of physical activity and computerized brain training improves verbal memory and increases cerebral glucose metabolism in the elderly. Translational psychiatry 2014, 4:e487.

25. Bherer L: Cognitive plasticity in older adults: effects of cognitive training and physical exercise. Annals of the New York Academy of Sciences 2015, 1337:1-6.

26. Iuliano E, di Cagno A, Aquino G, Fiorilli G, Mignogna P, Calcagno G, Di Costanzo A: Effects of Different Types of Physical Activity on the Cognitive Functions and Attention in Older People: A Randomised Controlled
Study. Experimental gerontology 2015.

27. Snow CM: Exercise Effects on Falls in Frail Elderly: Focus on Strength. . Journal of Applied Biomechanics 1999, 15:84-91.

28. Warburton DE, Nicol CW, Bredin SS: Health benefits of physical activity: the evidence. CMAJ : Canadian Medical Association journal = journal de l'Association medicale canadienne 2006, 174(6):801-809.

29. Caserotti P, Aagaard P, Larsen JB, Puggaard $L$ : Explosive heavy-resistance training in old and very old adults: changes in rapid muscle force, strength and power. Scand J Med Sci Sports 2008, 18(6):773-782.

30. Caserotti P, Aagaard P, Puggaard L: Changes in power and force generation during coupled eccentric-concentric versus concentric muscle contraction with training and aging. Eur J App/ Physiol 2008, 103(2):151-161.

31. Granacher $U$, Gollhofer A, Strass D: Training induced adaptations in characteristics of postural reflexes in elderly men. Gait Posture 2006, 24(4):459-466.

32. Granacher U, Gruber M, Gollhofer A: Force production capacity and functional reflex activity in young and elderly men. Aging Clin Exp Res 2009.

33. Granacher U, Muehlbauer T, Gruber M: A qualitative review of balance and strength performance in healthy older adults: impact for testing and training. Journal of aging research 2012, 2012:708905.

34. Hakkinen $\mathrm{K}$, Alen M, Kallinen M, Newton RU, Kraemer WJ: Neuromuscular adaptation during prolonged strength training, detraining and re-strength-training in middle-aged and elderly people. Eur J Appl Physiol 2000, 83(1):51-62.

35. Hakkinen K, Pakarinen A, Kraemer WJ, 
Hakkinen A, Valkeinen $\mathrm{H}$, Alen M: Selective muscle hypertrophy, changes in EMG and force, and serum hormones during strength training in older women. J App/ Physiol 2001, 91 (2):569-580.

36. Kramer AF, Hahn S, Cohen NJ, Banich MT, McAuley E, Harrison CR, Chason J, Vakil E, Bardell L, Boileau RA et al: Ageing, fitness and neurocognitive function. Nature 1999, 400(6743):418-419.

37. Landi F, Abbatecola AM, Provinciali M, Corsonello A, Bustacchini S, Manigrasso L, Cherubini A, Bernabei R, Lattanzio F: Moving against frailty: does physical activity matter? Biogerontology 2010, 11(5):537545.

38. Landi F, Marzetti E, Martone AM, Bernabei $\mathrm{R}$, Onder G: Exercise as a remedy for sarcopenia. Current opinion in clinical nutrition and metabolic care 2014, 17(1):2531.

39. Landi F, Onder G, Carpenter I, Cesari M, Soldato M, Bernabei R: Physical activity prevented functional decline among frail community-living elderly subjects in an international observational study. J Clin Epidemiol 2007, 60(5):518-524.

40. Landi F, Russo A, Barillaro C, Cesari M, Pahor M, Danese P, Bernabei R, Onder G: Physical activity and risk of cognitive impairment among older persons living in the community. Aging Clin Exp Res 2007, 19(5):410-416.

41. Lord SR, Castell S: Physical activity program for older persons: effect on balance, strength, neuromuscular control, and reaction time. Arch Phys Med Rehabil 1994, 75(6):648-652.

42. Lord SR, Castell S, Corcoran J, Dayhew J, Matters B, Shan A, Williams P: The effect of group exercise on physical functioning and falls in frail older people living in retirement villages: a randomized, controlled trial. J Am Geriatr Soc 2003, 51(12):1685-1692.

43. Lord SR, Lloyd DG, Nirui M, Raymond $J$, Williams P, Stewart RA: The effect of exercise on gait patterns in older women: a randomized controlled trial. J Gerontol A Biol Sci Med Sci 1996, 51(2):M64-70.

44. Rogan S, Baur $H$, Sargent A, Schori M, Taeymans J: [Feasibility of balance training on mats in healthy, moderately sportive women in old age : A pilot study.]. Z Gerontol Geriatr 2014.

45. Skelton DA: Effects of physical activity on postural stability. Age Ageing 2001, 30 Suppl 4:33-39.

46. Skelton DA, Beyer N: Exercise and injury prevention in older people. Scand $\mathrm{J}$ Med Sci Sports 2003, 13(1):77-85.

47. Steib S, Schoene D, Pfeifer K: Doseresponse relationship of resistance training in older adults: a meta-analysis. Med Sci Sports Exerc 2010, 42(5):902-914.

48. Taube W, Gruber M, Gollhofer A: Spinal and supraspinal adaptations associated with balance training and their functional relevance. Acta Physiol (Oxf) 2008, 193(2):101-116.

49. Skelton D, Dinan S: Exercise for falls management: Rationale for an exercise programme aimed at reducing postural instability. Physiotherapy Theory and Practice 1999, 15(2):105-120.

50. Gill TM, Williams CS, Tinetti ME: Assessing risk for the onset of functional dependence among older adults: the role of physical performance. J Am Geriatr Soc 1995, 43(6):603-609.

51. Guralnik JM, Ferrucci L, Simonsick EM, 
Salive ME, Wallace RB: Lower-extremity function in persons over the age of 70 years as a predictor of subsequent disability. $N$ Engl J Med 1995, 332(9):556-561.

52. Studenski S, Perera S, Wallace D, Chandler JM, Duncan PW, Rooney E, Fox M, Guralnik JM: Physical performance measures in the clinical setting. J Am Geriatr Soc 2003, 51(3):314-322.

53. Zeyfang A, Braun A: [Guidelines „Diabetes mellitus in the elderly"]. MMW Fortschr Med 2009, 151(20):33-35, 37.

54. Kessler J, Radlinger L, Baur H, Rogan S: Effect of stochastic resonance whole body vibration on functional performance in the frail elderly: A pilot study. Arch Gerontol Geriatr 2014, 59(2):305-311.

55. Rogan S, Schmidtbleicher D, Radlinger L: Immediate effects after stochastic resonance whole-body vibration on physical performance on frail elderly for skilling-up training: a blind cross-over randomised pilot study. Aging Clin Exp Res 2014, 26(5):519-527.

56. Daniel K: Wii-hab for pre-frail older adults. Rehabilitation nursing : the official journal of the Association of Rehabilitation Nurses 2012, 37(4):195-201.

57. Szturm T, Betker AL, Moussavi Z, Desai A, Goodman V: Effects of an interactive computer game exercise regimen on balance impairment in frail communitydwelling older adults: a randomized controlled trial. Physical therapy 2011, 91(10):1449-1462.

58. Bogaerts AC, Delecluse C, Claessens AL, Troosters T, Boonen S, Verschueren SM: Effects of whole body vibration training on cardiorespiratory fitness and muscle strength in older individuals (a 1-year randomised controlled trial). Age Ageing 2009, 38(4):448-454

59. de Bruin ED, van Het Reve E, Murer K: A randomized controlled pilot study assessing the feasibility of combined motor-cognitive training and its effect on gait characteristics in the elderly. Clin Rehabil 2012.

60. Furness TP, Maschette WE, Lorenzen C, Naughton GA, Williams MD: Efficacy of a whole-body vibration intervention on functional performance of communitydwelling older adults. J Altern Complement Med 2010, 16(7):795-797.

61. Haas CT, Turbanski S, Kessler K, Schmidtbleicher D: The effects of random whole-body-vibration on motor symptoms in Parkinson's disease. NeuroRehabilitation 2006, 21(1):29-36.

62. Merriman $\mathrm{H}$, Jackson $\mathrm{K}$ : The effects of whole-body vibration training in aging adults: a systematic review. J Geriatr Phys Ther 2009, 32(3):134-145.

63. Mikhael M, Orr R, Amsen F, Greene D, Singh MA: Effect of standing posture during whole body vibration training on muscle morphology and function in older adults: a randomised controlled trial. BMC Geriatr 2010, 10:74.

64. Pichierri G, Coppe A, Lorenzetti S, Murer K, de Bruin ED: The effect of a cognitive-motor intervention on voluntary step execution under single and dual task conditions in older adults: a randomized controlled pilot study. Clinical interventions in aging 2012, 7:175-184.

65. Rees SS, Murphy AJ, Watsford ML: Effects of whole body vibration on postural steadiness in an older population. $J \mathrm{Sci}$ Med Sport 2009, 12(4):440-444.

66. Schoene D, Valenzuela T, Lord SR, de Bruin 
ED: The effect of interactive cognitive-motor training in reducing fall risk in older people: a systematic review. BMC Geriatr 2014, 14:107.

67. Turbanski S, Haas CT, Schmidtbleicher D, Friedrich A, Duisberg P: Effects of random whole-body vibration on postural control in Parkinson's disease. Res Sports Med 2005, 13(3):243-256.

68. van Nes IJ, Geurts AC, Hendricks HT, Duysens J: Short-term effects of whole-body vibration on postural control in unilateral chronic stroke patients: preliminary evidence. Am J Phys Med Rehabil 2004, 83(11):867-873

69. van Nes IJ, Latour $H$, Schils F, Meijer R, van Kuijk A, Geurts AC: Long-term effects of 6-week whole-body vibration on balance recovery and activities of daily living in the postacute phase of stroke: a randomized, controlled trial. Stroke 2006, 37(9):23312335.

70. von Stengel S, Kemmler W, Engelke K, Kalender WA: Effects of whole body vibration on bone mineral density and falls: results of the randomized controlled ELVIS study with postmenopausal women. Osteoporos Int 2011, 22(1):317-325.

71. Wuest $S$, Borghese NA, Pirovano $M$, Mainetti $\mathrm{R}$, van de Langenberg $\mathrm{R}$, de Bruin ED: Usability and Effects of an ExergameBased Balance Training Program. Games Health J 2014, 3(2):106-114.

72. Machado A, Garcia-Lopez D, GonzalezGallego J, Garatachea N: Whole-body vibration training increases muscle strength and mass in older women: a randomizedcontrolled trial. Scand J Med Sci Sports 2010, 20(2):200-207.

73. Marin PJ, Herrero AJ, Sainz N, Rhea
MR, Garcia-Lopez D: Effects of different magnitudes of whole-body vibration on arm muscular performance. $J$ Strength Cond Res 2010, 24(9):2506-2511.

74. Osawa Y, Oguma Y: Effects of wholebody vibration on resistance training for untrained adults. Journal of sports science \& medicine 2011, 10(2):328-337.

75. Ronnestad BR: Acute effects of various whole body vibration frequencies on $1 \mathrm{RM}$ in trained and untrained subjects. J Strength Cond Res 2009, 23(7):2068-2072.

76. Haas CT, Schmidtbleicher D: About effects of exercise and stochastice resonance on neuroplasticity and neuroptrotection In: Current results of strength training researcha multi-perspective approach. Edited by Gießing J F, M, vol. 2. Göttingen, Germany: Cuvillier Verlag; 2008: 105-118.

77. Haas CT, Turbanski S, Kaiser I, Schmidtbleicher D: [Biomechanical and physiological effects of oscillating mechanical stimuli in humans]. Deutsche Zeitschrift für Sportmedizin 2004, 55(2):3443.

78. Haas CT, Turbanski S, Markitz S, Kaiser I, SchmidtbleicherD: StochastischeResonanz in der Therapie von Bewegungsstörungen. Bewegungsstherapie und Gesundheitssport 2006, 22:58-61.

79. Priplata AA, Patritti BL, Niemi JB, Hughes R, Gravelle DC, Lipsitz LA, Veves A, Stein $\mathrm{J}$, Bonato P, Collins JJ: Noise-enhanced balance control in patients with diabetes and patients with stroke. Ann Neurol 2006 , 59(1):4-12

80. Pichierri G, Wolf $P$, Murer K, de Bruin ED: Cognitive and cognitive-motor interventions affecting physical functioning: a systematic review. BMC Geriatr 2011, 11:29. 
81. Pichierri G, Murer K, de Bruin ED: A cognitive-motor intervention using a dance video game to enhance foot placement accuracy and gait under dual task conditions in older adults: a randomized controlled trial. BMC Geriatr 2012, 12:74.

82. de Bruin ED, Schoene D, Pichierri G, Smith ST: Use of virtual reality technique for the training of motor control in the elderly. Some theoretical considerations. Z Gerontol Geriatr 2010, 43(4):229-234.

83. Guralnik JM, Branch LG, Cummings SR, Curb JD: Physical performance measures in aging research. J Gerontol 1989, 44(5):M141-146.

84. Reuben DB, Siu AL, Kimpau S: The predictive validity of self-report and performance-based measures of function and health. J Gerontol 1992, 47(4):M106110.

85. Guralnik JM, Winograd $\mathrm{CH}$ : Physical performance measures in the assessment of older persons. Aging (Milano) 1994, 6(5):303-305.

86. Freire AN, Guerra RO, Alvarado B, Guralnik JM, Zunzunegui MV: Validity and reliability of the short physical performance battery in two diverse older adult populations in Quebec and Brazil. J Aging Health 2012, 24(5):863-878.

87. Derlien S, Böhme B, Leistritz L, Smolenski UC: Validitätsuntersuchung zum neuen, innovativen Ganganalysesystem RehaWatch von Hasomed. Manuelle Medizin 2010, 48(4):254-259.

88. Schwesig R, Leuchte S, Fischer D, Ullmann $R$, Kluttig A: Inertial sensor based reference gait data for healthy subjects. Gait \& posture 2011, 33(4):673-678. 



\section{Chapter 2}

Effects of whole-body vibration

on postural control in elderly:

a systematic review and meta-analysis 


\section{ABSTRACT}

\section{Background}

This systematic review was performed to summarize the current evidence for whole body vibration (WBV) interventions on postural control in elderly.

\section{Methods}

English and German language papers in Medline, PEDro, Cinahl and the Cochrane databases were searched. Two reviewers extracted data on patients' characteristics, type of WBV intervention and outcomes. Two independent reviewers rated the methodological quality of these studies. Data were pooled using random-effects meta-analysis.

\section{Results}

Fifteen papers reporting quantitative data were included. Results from 15 papers could be pooled for a meta-analysis. The studies involved 933 participants. In 7 studies the authors investigated the effects of vibration plates generating vertical sinusoidal vibrations (VS-WBV) and 7 papers described the use of side-alternating sinusoidal vibrations (SS-WBV). One study investigated both VS-WBV and SS-WBV.

Weak to moderate evidence of an overall effect as a result of VS-WBV and SS-WBV was observed for (a) static balance for post-intervention values with a standardized mean difference (SMD) $-0.06,95 \% \mathrm{Cl}-0.31$ to 0.18 and for change values SMD $-0.26,95 \% \mathrm{Cl}-1.09$ to 0.57 , and (b) dynamic balance for post-intervention-values SMD $-0.34,95 \% \mathrm{Cl}-0.60$ to -0.08 . For functional balance (c) an overall outcome for post-intervention values with SMD of $0.34,95 \% \mathrm{Cl}-0.19$ to 0.87 was found.

\section{Conclusions}

The 15 studies reviewed were of moderate methodological quality. In summary, SS-WBV seems to have a beneficial effect on dynamic balance in elderly individuals. However, the current results should be interpreted with caution because of the observed heterogeneity of training parameters and statistical methods. Future studies are warranted to evaluate the effects of WBV on postural control in an elderly population. 


\section{Background}

Even in the absence of overt pathology, motor functioning [cf. International Classification of Functioning (ICF) (see http://www.who.int/classification/icf)] can deteriorate, as is illustrated by more frequent falls in ageing populations [2]. Usually multi-factorial disorders such as impaired vision, vestibular dysfunction, sensory loss, muscular weakness or gait disorders contribute to more frequent falls [3-6]. Falls are amongst the most common reasons for medical intervention in the elderly and their occurrence may initiate a vicious circle that causes fear of falling, nursing home admittance and loss of independence [7]. About 30\% of the elderly fall at least once a year, while one-fifth of these need medical care [8].

Because a significant portion of the older population is unable or unwilling to comply with conventional training regimens [9], there seems to be a need for a search and assessment of alternative forms of training intervention contents. More recently, whole body vibration (WBV) training has been widely used in fitness centers, sports, and physical therapy to improve cardiorespiratory fitness, power and strength [10-17] or bone mineral density [18-22]. Several studies have demonstrated that WBV also improves postural control in healthy young or elderly individuals as well as in patients with orthopedic (e.g. rupture of the cruciate ligaments) [16] or neurological diseases (e.g. Parkinson`s disease, multiple sclerosis, spinal cord injury) [15, 23, 24]. Torvinen et al. [25] showed beneficial long-term effects of sinusoidal WBV on the strength of young healthy individuals but not immediately on postural control. For patients with Parkinson `s disease Haas and Schmidtbleicher postulated [26] that stochastic resonance whole-body vibration (SR-WBV) transiently improves balance.

However, while most WBV studies demonstrate significant improvements in balance, these results must be interpreted with caution. Many of the published studies have methodological flaws such as the questionable validity of the outcome measures used or the absence of a sham intervention [27]. The results of the studies are not completely consistent, and some reports found only little or no effect on postural control following WBV training [19, 25, 28, 29]. One explanation for the contradictory results could be attributed to the inconsistent training parameters used for WBV training. The frequency [12], amplitude, duration of one vibration session, and the number of vibration interventions, are the treatment parameters that need to be considered when using WBV. The duration of rest periods between vibration sessions also seems to play an important role $[30,31]$. It is, furthermore, very difficult to determine an optimal training strategy because the underlying mechanisms contributing to improved balance after WBV have so far not been clarified. Most of the devices used vibrate sinusoidally while one system generates stochastic vibrations. The devices which induce sinusoidal vibrations have subjects standing on one platform and they either oscillate purely vertically (VS-WBV) or sidealternating (SS-WBV). The WBV device that vibrates in a stochastic manner (SR-WBV) exhibits separate platforms for each foot. 
The purpose of this systematic review is to provide an overview of the current available evidence for the use of WBV to improve balance in elderly individuals. In particular the following aspects should be clarified: a) assessment of the quality and internal validity of the included studies, b) description of the assessments used to document the effects of WBV on balance, c) composition of the WBV training parameters in relation to the different vibration plates, and d) conclusion about the clinical relevance. Furthermore this review should give some more conclusive results about the effects of WBV on the balance skills of elderly by summarizing the available studies in a meta-analysis.

\section{Methods}

\section{Data Sources and Searches}

The methods of the analysis and the inclusion criteria were developed and documented in a protocol prior to the actual review. This protocol can be found in additional file 1. PRISMA guidelines were followed for this systematic review and meta-analysis [32]. An electronic search of the following databases up until May 2011 was conducted: PubMed, Cochrane Register of Controlled Trials, Physiotherapy Evidence Database (PEDro) and CINAHL (Ebsco Host). The unpublished International Clinical Trials Registry Platform from the World Health Organization (WHO) was also searched. Furthermore, a manual search was completed within the reference lists of retrieved publications.

\section{Study Selection and Research Question}

This systematic review was conducted to answer the question formulated according recommendations from the PICO-model, where the acronym PICO stands for Population (in the

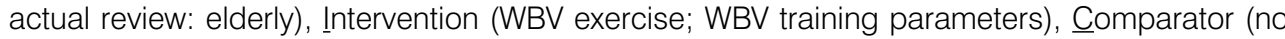
or other balance enhancing exercise) and Outcomes (examination of postural control; static, dynamic, functional and balance; falls) [33].

\section{The following keywords were used for formulating the search strategy of our review:}

Population: elderly, aged, dwelling home, nursing home Intervention: Whole-body vibration, WBV, noise, random vibration

Outcome: Balance, postural stability, postural control, sway, falls In the case of missing data additional information was requested from the corresponding authors of relevant papers in order to include these data in our meta-analysis.

Three independent reviewers ( $\mathrm{SR}, \mathrm{KH}, \mathrm{RH})$ screened the titles and abstracts for eligibility. We were aiming at Randomized Controlled Trials (RCT) measuring postural control/balance in studies using WBV intervention in elderly subjects. Both published and unpublished (grey literature) full text articles in English or German were eligible for inclusion. Elderly participants 
and all clinical outcome measures of static, dynamic and functional balance performance as well as computerized biomechanical assessments of postural control (e.g. posturography) were included in this review. Detailed descriptions of the different balance tests are reported elsewhere [34-37]. The following types of articles were excluded: studies describing vibrations applied by current or vibrating insoles and conference papers.

\section{Data Extraction and Quality Assessment}

The methodological quality of the included articles was rated with "The Cochrane Collaboration's tool for assessing risk of bias". The criteria list comprised six items. Each item was scored with "+" if the criterion was fulfilled, with "-" if the criterion was not fulfilled, and with "?" if the information was not provided or was unclear. All included papers were scored independently by three reviewers $(\mathrm{KH}, \mathrm{RH}, \mathrm{SR})$. Discrepancies were resolved by discussion and consensus.

In addition, general characteristics of the studies were extracted. Two authors ( $\mathrm{KH}, \mathrm{SR})$ independently abstracted the following information from each of the studies included in this review: 1) design and sample; 2) inclusion criteria; 3) training parameters 4) type of vibration plate; 5) change in static, dynamic and functional balance and 6) conclusions of the studies and statistical significance.

\section{Data Synthesis and Analysis}

All outcomes of interest were presented as continuous data (mean values and SD or mean changes). We used standardized mean difference (SMD), except for the analysis of the Timed up and Go test (TUG) where we used weighted mean difference (WMD). Random effects models were applied. The magnitude of the effect size SMD can be rated as follows: 0.2 indicates a small effect, 0.5 a moderate effect and 0.8 a large effect [38]. Where only one study was identified or data were not eligible to be included in the meta-analysis, results of individual studies are presented.

To determine the isolated effect of WBV on posture we additionally performed a sensitive analysis for dynamic balance where studies with WBV in combination with exercise were compared to studies with isolated WBV interventions.

If studies reported more than one balance assessment, the primary outcome of a study was chosen and included in the meta-analysis.

Heterogeneity was assessed by forest plots and the $\mathrm{I}^{2}$ statistics. Values $>25 \%$ indicate small, $>$ $50 \%$ middle and $>75 \%$ considerable heterogeneity [39]. All other information was summarized and analyzed qualitatively.

For all calculations Stata (Version12) was used. 


\section{Results}

\section{Study characteristics}

The literature search revealed a total of 95 possibly eligible papers (PubMed: $n=88$, Cochrane: $n=30$, CINAHL: $n=12$, PEDro: $n=13$, and Unpublished International Clinical Trials Registry Platform: $n=33$ ). The title and abstracts of these 95 papers were studied and after removing duplicates 71 studies remained for further analysis. The majority of these papers had to be excluded $(n=56)$ because they did not refer to postural control or used electrical vibration stimuli. Finally 15 full text papers could be included in the present meta-analysis [19, 22, 40-52]. 7 papers reported vertical sinusoidal WBV (VS-WBV) and 7 papers side-alternating sinusoidal WBV (SS-WBV). One study reported on a combined VS-WBV and SS-WBV intervention [52]. For SR-WBV, no RCT studies could be identified.

A flow diagram of the search process is shown in Figure 1.

\section{Study Design and Characteristics of Population}

A broad quality spectrum within the RCT study design and heterogeneity within the applied training strategies was found. The major characteristics of the 15 selected papers are summarized in Table 1.

Studies were conducted in Australia (6), Belgium (5), Italy (1), Hong Kong (1), Spain (1), and the USA (1). 


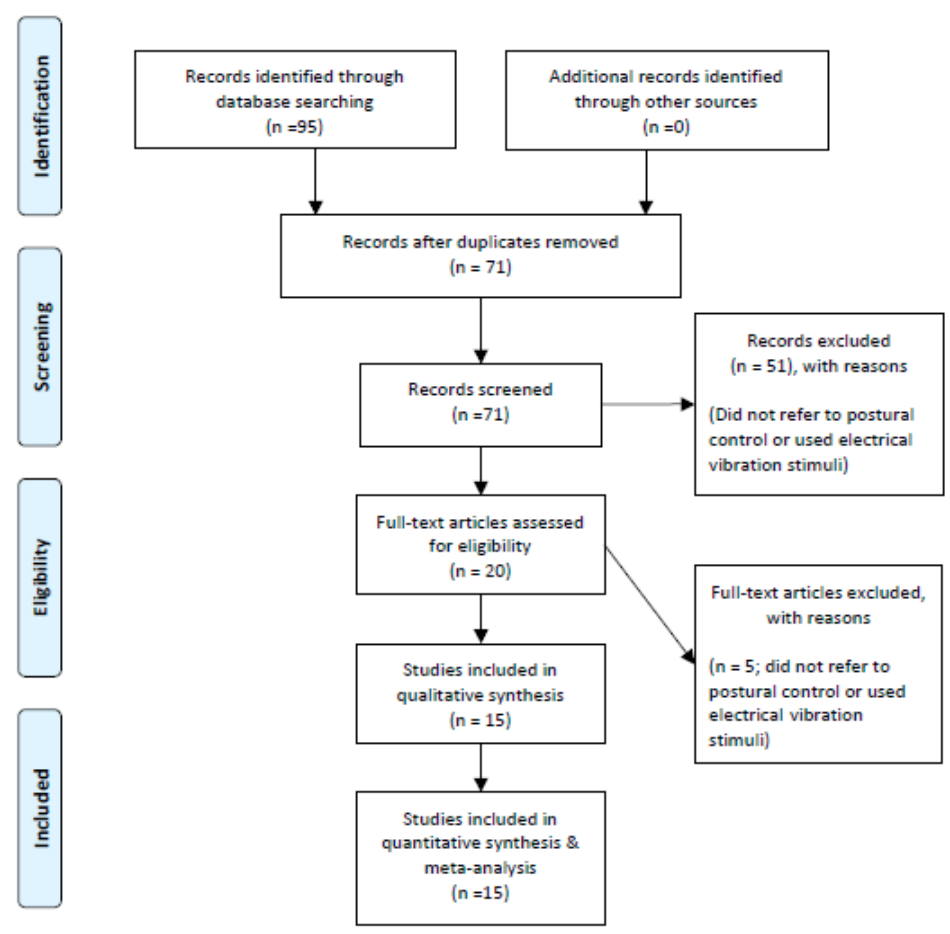

\section{Figure 1}

Results of th-e systematic review

\section{Methodological Quality}

All included studies summarized in table 2 showed a bias following the "Cochrane Collaboration's tool for assessing risk of bias". Most of them had a very high risk for "allocation concealment", "blinding", and for "incomplete outcome data". Only four of the included studies [42, 45, 47, 49] provided some information about randomization procedures, suggesting that randomization was probably concealed. Two studies $[41,45]$ report a blind assessor incorporated for all outcome measures. Most studies had a low risk for "incomplete data" and "other bias". 


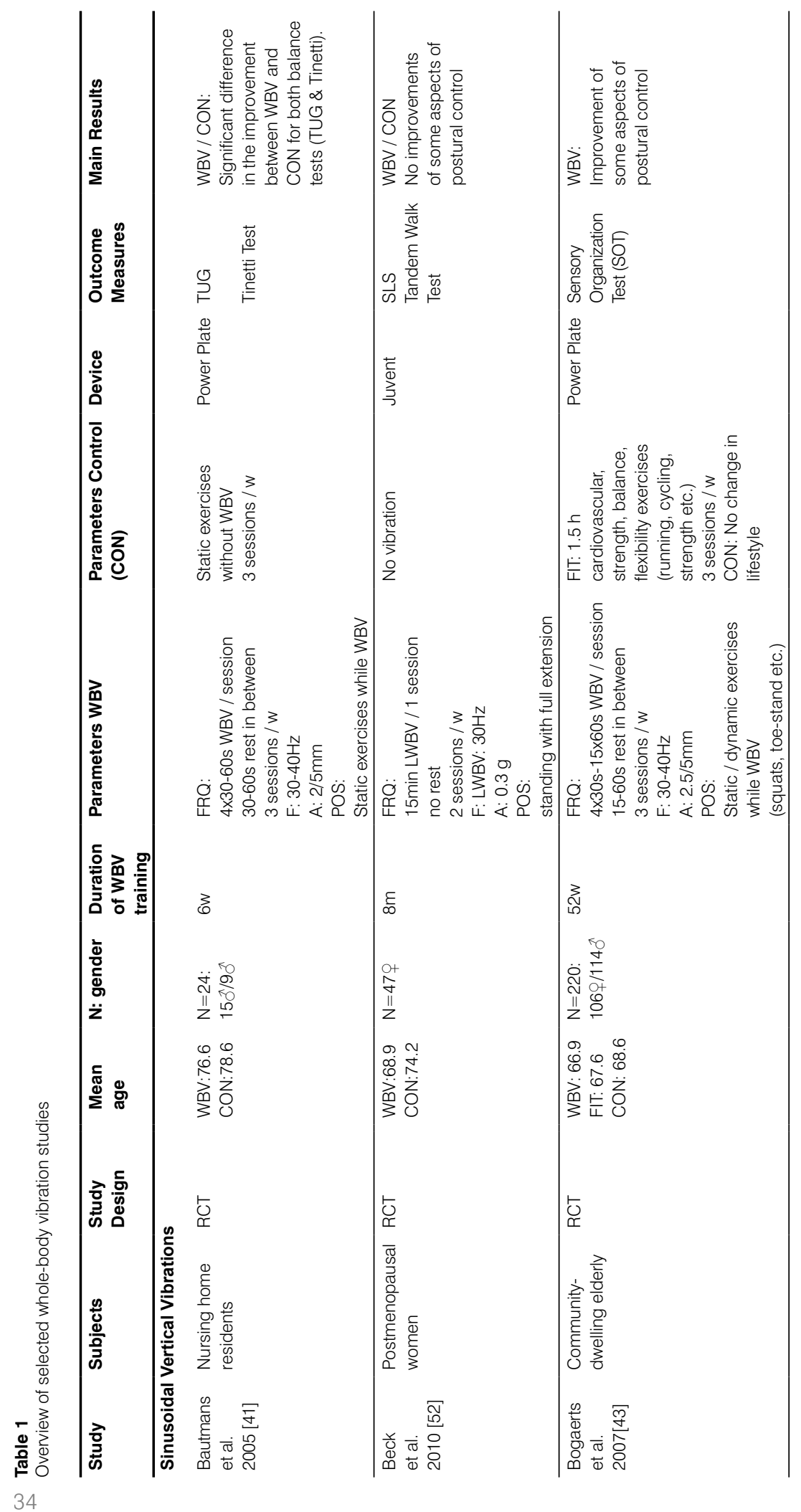




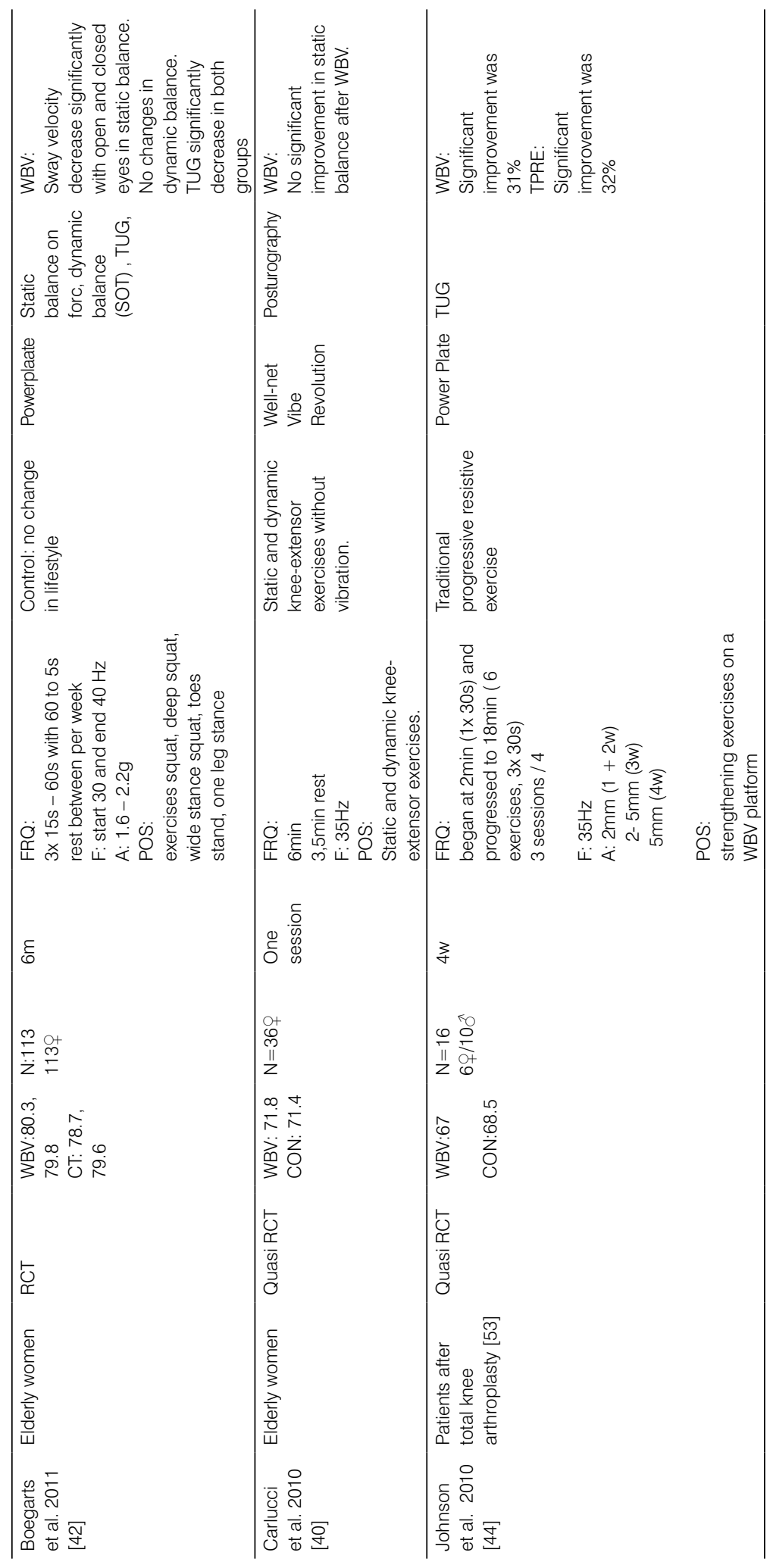




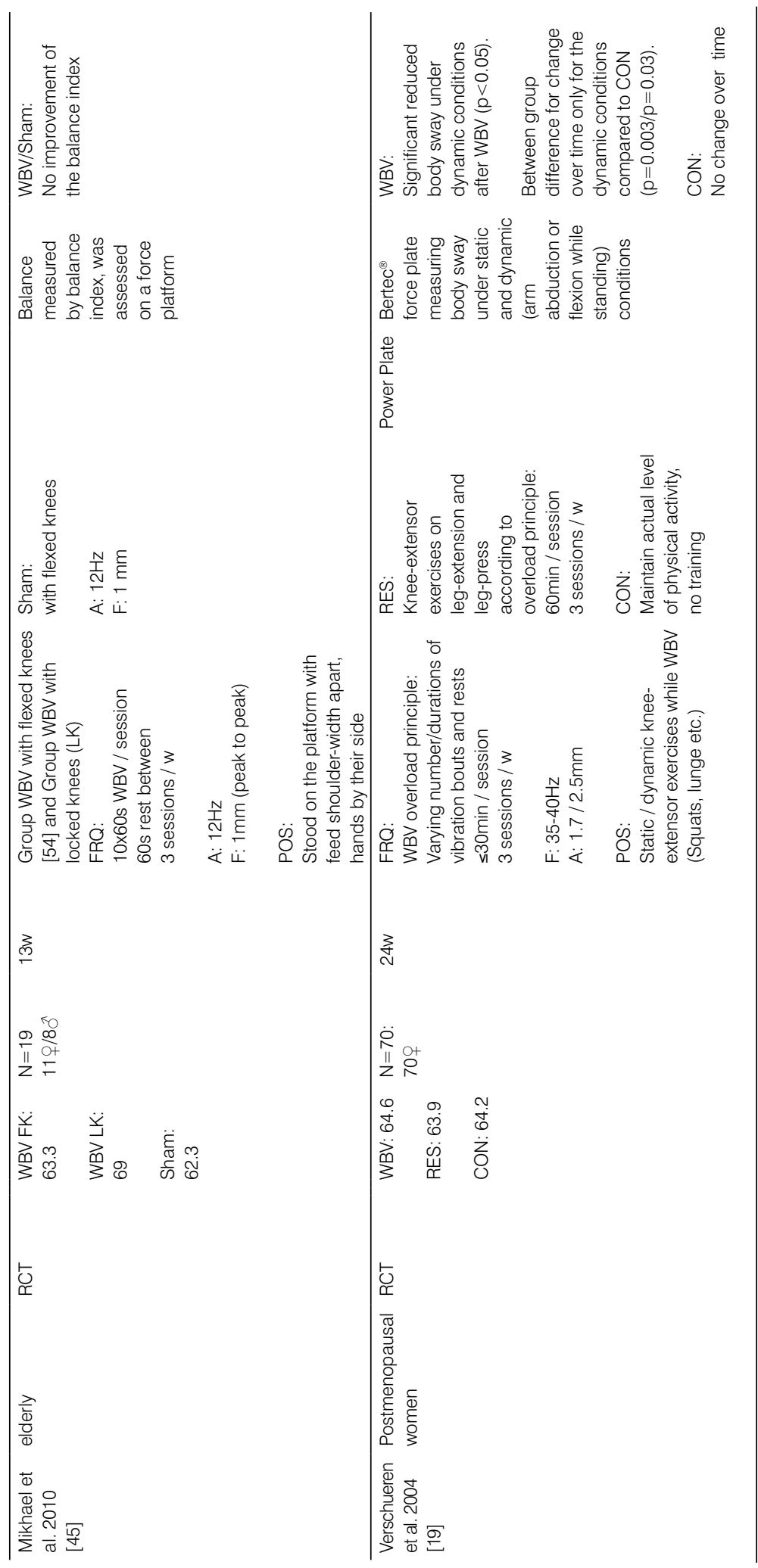




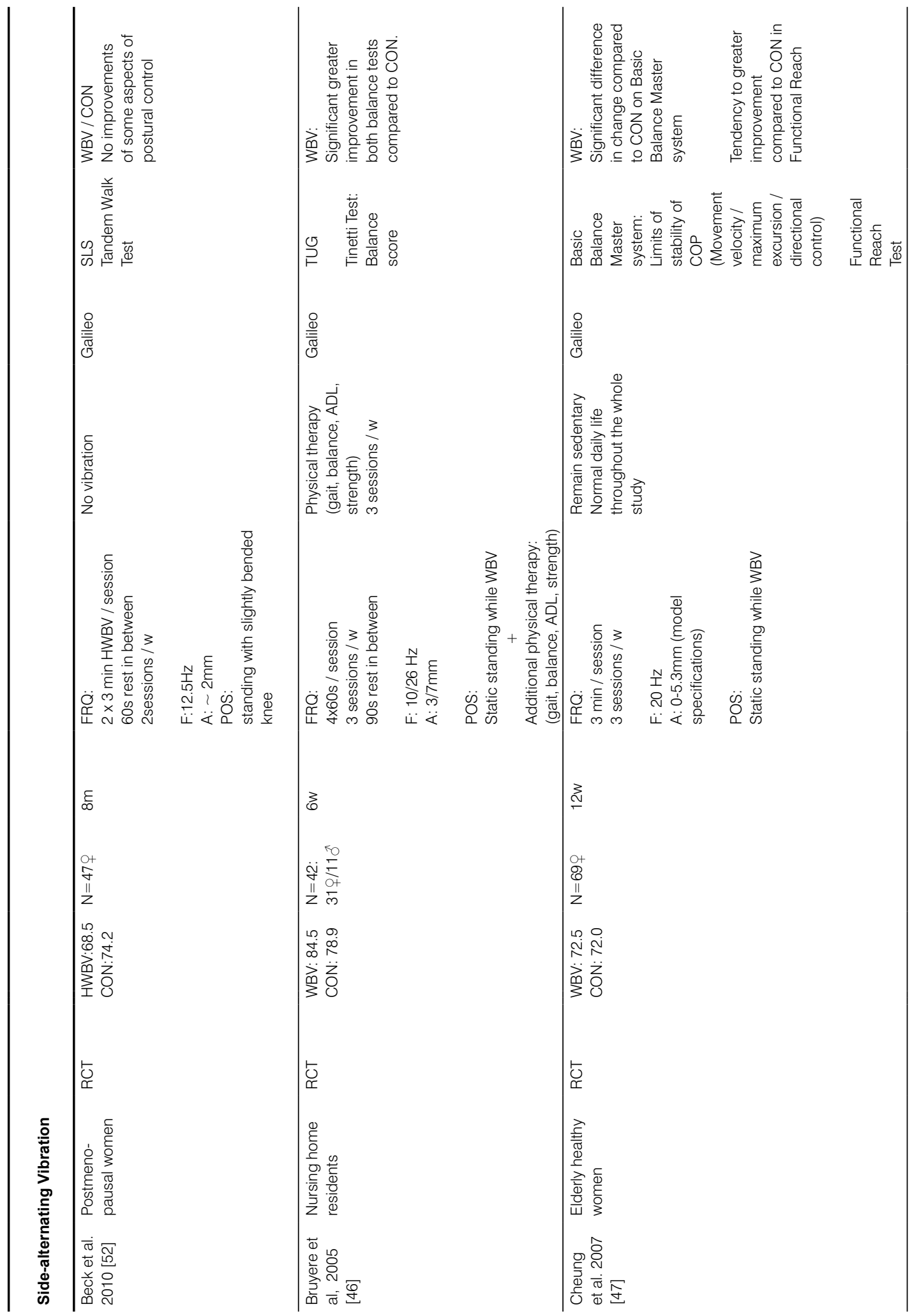




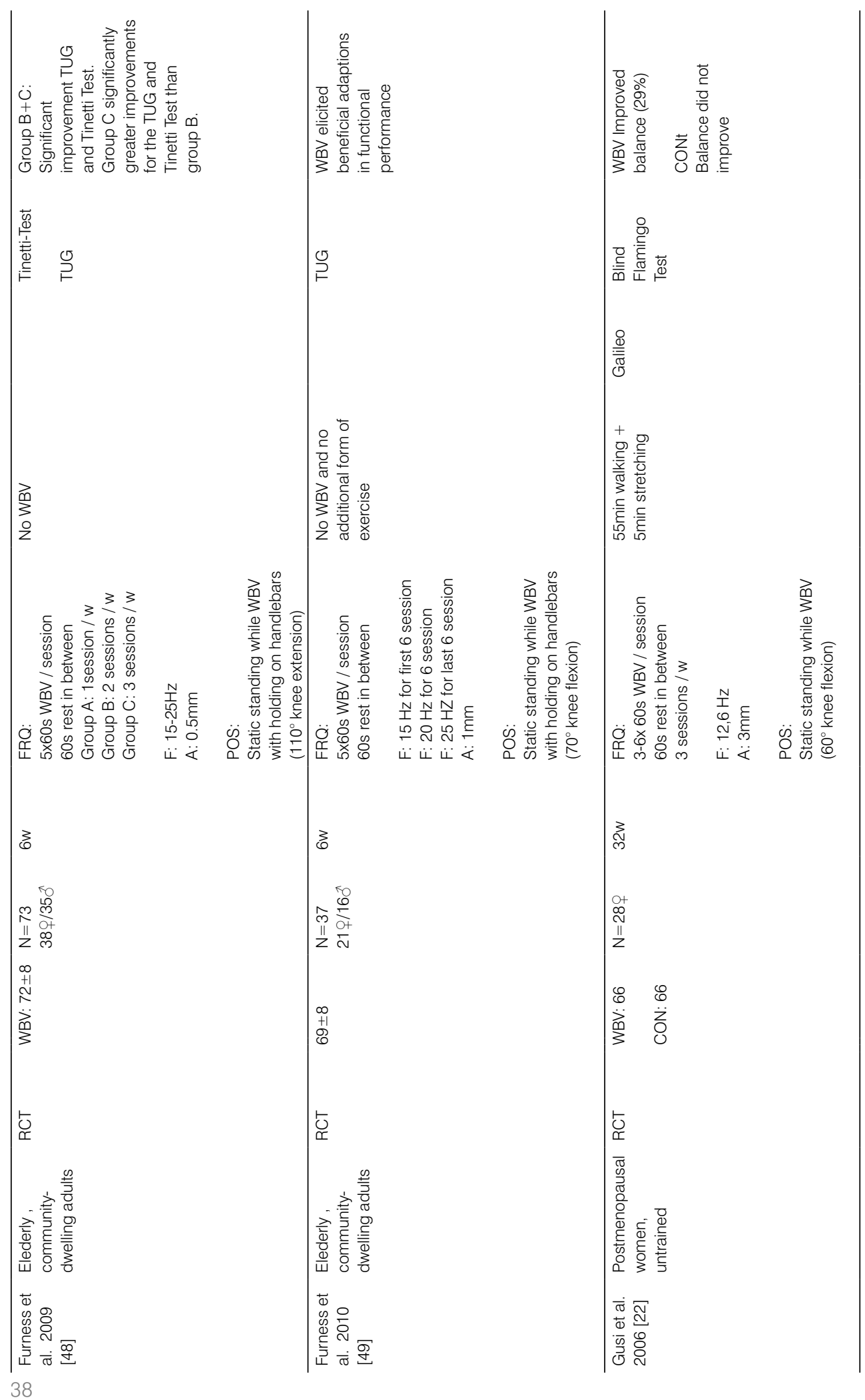




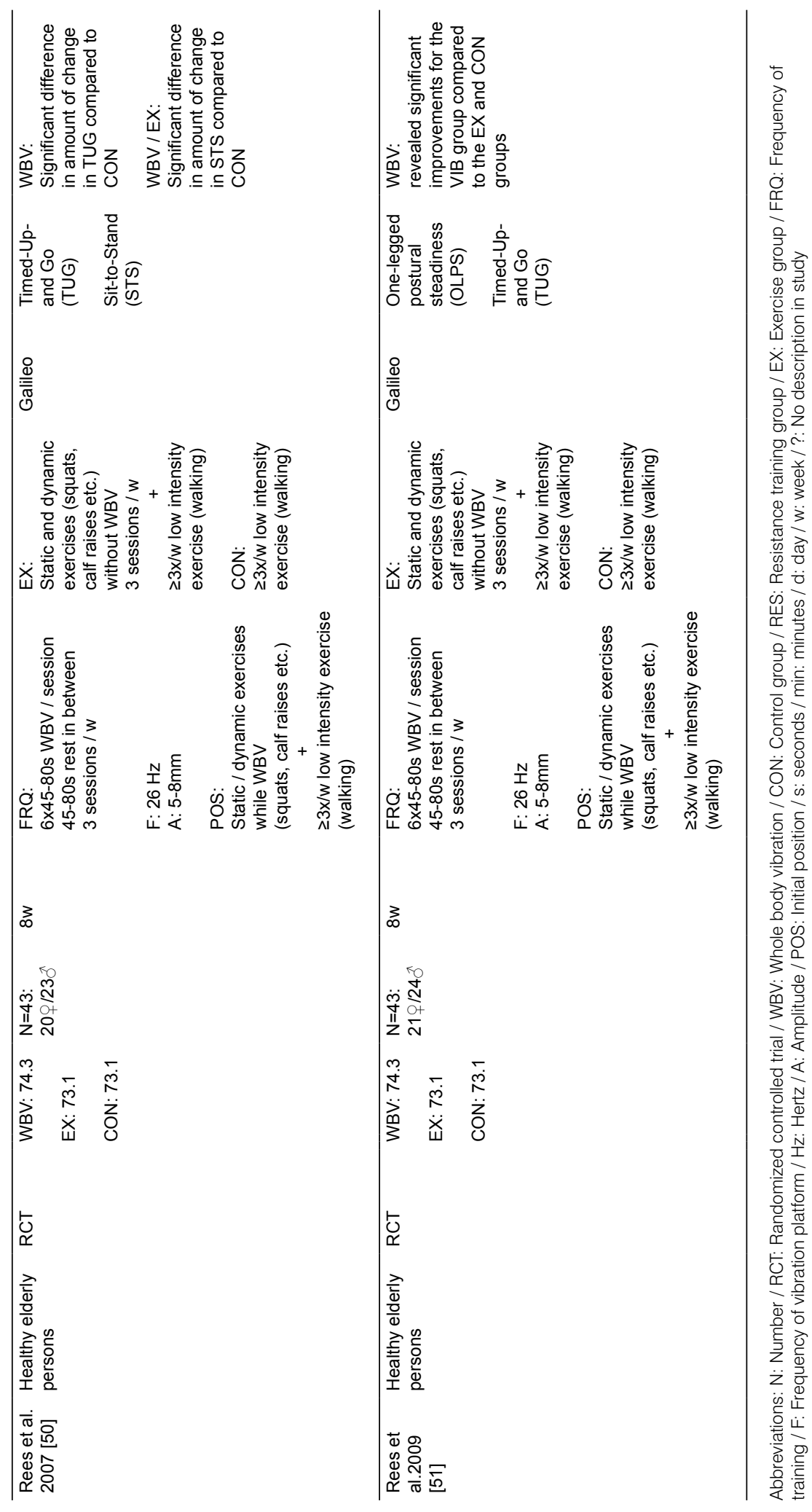


Table 2

Methodological quality of included studies

\begin{tabular}{|c|c|c|c|c|c|c|}
\hline Study & RCT & $\begin{array}{l}\text { Allocation } \\
\text { Concealed }\end{array}$ & Blinding & $\begin{array}{l}\text { Incomplete } \\
\text { data } \\
\text { addressed }\end{array}$ & $\begin{array}{l}\text { Free of } \\
\text { selective } \\
\text { reporting }\end{array}$ & $\begin{array}{l}\text { Free of } \\
\text { other } \\
\text { bias }\end{array}$ \\
\hline Bautmans et al. 2005 [41] & + & - & + & + & + & + \\
\hline Beck et al. 2010 [52] & + & . & & + & + & $?$ \\
\hline Bogaerts et al. 2007 [43] & + & - & - & - & + & + \\
\hline Boegarts et al. 2011[42] & + & + & - & + & + & + \\
\hline Carlucci et al. 2010 [40] & quasi RCT & - & - & + & + & + \\
\hline Johnson et al. 2010[44] & quasi RCT & - & - & + & + & + \\
\hline Mikhael et al. 2010 [45] & + & + & + & + & + & + \\
\hline Verschueren et al. 2004 [19] & + & - & $?$ & + & + & + \\
\hline Bruyere et al. 2005 [46] & + & - & - & + & + & + \\
\hline Cheung et al. 2007 [47] & + & + & - & - & + & - \\
\hline Furness et al. 2009 [48] & + & $?$ & - & + & + & - \\
\hline Furness et al. 2010 [49] & + & + & - & + & + & $?$ \\
\hline Gusi et al.2006 [22] & + & - & - & - & + & + \\
\hline Rees et al. 2007 [50] & + & - & - & - & + & + \\
\hline Rees et al. 2009 [51] & + & - & - & - & + & + \\
\hline
\end{tabular}

Abbreviations: +: criterion was fulfilled / -: the criterion was not fulfilled / ?: the information was not provided or was unclear

\section{Training Protocols}

Table 1 shows WBV training protocols of the included studies (Table 1). Several differences were seen in the WBV training protocols. Treatment parameters for VS-WBV revealed frequencies ranging between 12 to $40 \mathrm{~Hz}$. The most common mean frequency was about $30 \mathrm{~Hz}$. A training session usually consisted of 3 to 10 series of 30-60 seconds WBV with a rest of 30 to 60 seconds in between. Beck et al. [52] described one single session lasting 15 minutes without rest. For long-term interventions the authors often prescribed 3 WBV sessions per week. Verschueren et al. [19] used variable numbers of sessions per week. The duration of long-term WBV lasted from 6 to 52 weeks. The initial position the subjects had to adopt during WBV differed between the studies. One author prescribed standing upright statically with feet shoulder-width apart and hands at the side [45]. In a further six studies [40-44, 52, 55] the participants had to perform various dynamic physical exercises during WBV (e.g. squatting exercises).

Treatment parameters for SS-WBV revealed frequencies ranging between 5 and $26 \mathrm{~Hz}$. A training session usually consisted of 3 to 10 series of 30-60 seconds WBV with 60 seconds rest in between. Cheung et al. [47] did not describe the vibration session in detail (time of intervention / rest). Bruyere et al. [46] used 90 seconds, and Rees [50] used 45 to 80 seconds rest time between the vibration bouts. Frequency of training was 3 sessions per week for long-term intervention. Furness et al. [48] described three different vibration groups which trained 1, 2 or 
3 times per week. The duration of the whole WBV intervention varied between 6 to 32 weeks. Comparable with the VS-WBV studies, the starting positions of the subjects on the vibration devices varied. In most of the studies the participants stood in an upright position with slightly bent knees. Two studies prescribed dynamic exercises during WBV [50, 51].

\section{Outcome Measures}

In total, 10 different outcome measures for postural control/balance could be found in the 15 studies. For VS-WBV, different assessments for static balance (Posturography on force plates, Balance index, single leg stand), for dynamic balance (Timed Up and Go (TUG) Test, Sensory Organization Test (SOT), Tandem walk test) and for functional balance (Tinetti Test/ POMA) could be found.

One RCT reported no improvement in static and dynamic balance after WBV. In this study subjects were not expected to perform active exercises whilst standing on the vibration plate. Two RCTs $[19,42]$ observed improved static balance after WBV combined with simultaneous dynamic exercises. One RCT [45] reported no improvement after vibration without exercise in the balance index. Three RCTs [41-43] described improvement in dynamic balance following a combination of vibration with exercise. One RCT [41] reported improvement in functional balance following vibration bouts that were combined with exercise.

For the studies that applied SS-WBV several outcome measures were used. Three assessments for static balance (Basic Balance Master System, Blind Flamingo, single leg stand), 4 for dynamic balance (Functional Reach Test [FRT], Timed Up and Go [TUG] Test, chair rising, tandem walk test) and 1 for functional balance (POMA) were described.

Two RCTs $[22,47]$ reported improvements in static balance of the WBV participants. These improvements were achieved without performing additional dynamic exercises during WBV. Five RCTs [46-50] showed improved balance in subjects of the WBV group which was not obligated to perform simultaneous active exercises. Two RCTs $[50,51]$ demonstrate improvements in dynamic balance after a combination of WBV with additional exercises on the vibration platform. Two RCTs $[46,48]$ report improvements in functional balance after WBV without additional exercises. One study found no improvements in neither static nor dynamic balance after isolated WBV [52].

\section{Meta-analysis}

For the meta-analyses, 15 studies were included which described static, dynamic or functional balance outcome measurements. The effect sizes for these outcomes are summarized in Figures 2, 3, 4, 5 and 6. 


\section{Static balance}

The mean overall effect size for post-intervention values of static balance was SMD -0.06 (95\% Cl -0.31 to 0.18 ) and for change values SMD -0.26, 95\% Cl -1.09 to 0.57 (SS-WBV) (Figure 2). Postintervention values for dynamic balance was SMD -0.34 (95\% Cl -0.60 to -0.08$)$ (Figure 4), and for functional balance an overall outcome for post-intervention values revealed a SMD of 0.34 , $95 \% \mathrm{Cl}-0.19$ to 0.87 (additional file 2). There was small heterogeneity for post-intervention values for static balance with $1^{2} 0.00 \%(p=0.896)$, middle heterogeneity with $1^{2} 73.2 \%(p=0.024)$ for change values, and small heterogeneity for post-interventional values in dynamic balance with $l^{2}$ $42.6 \%(p=0.083)$. For functional balance no heterogeneity with $\left(1^{2} 3.4 \%, p=0.309\right)$ was found.

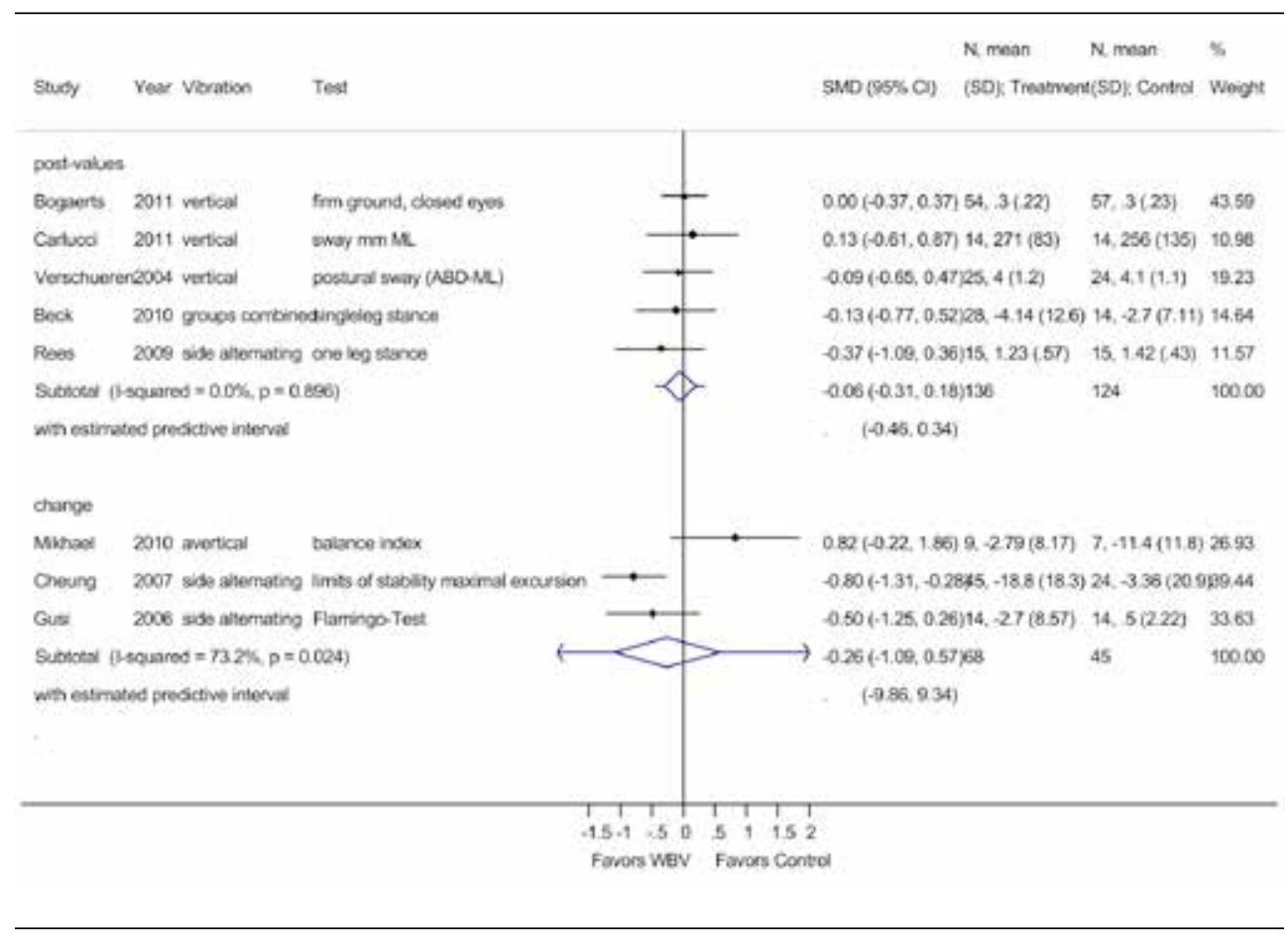

\section{Figure 2}

Forest plot of 8 trials comparing the effects of any type of vibration and control interventions on static balance. The analyses were separated for trials reporting post-values (i.e. mean and SD from follow-up) and for trials that reported change values (i.e. mean and SD from the changes from baseline to follow-up). Random effects model with predictive interval. The predictive interval indicates the range within which we expect the effects of $95 \%$ of future studies. Values on $x$-axis denote SMDs. 
For subgroup analysis, variables were pooled separately for VS-WBV and SS-WBV. Four VS-WBV studies showed post intervention mean values indicating a small effect size (SMD -0.02, 95\% $\mathrm{Cl}-0.28$ to 0.25 ) for static balance (Figure 3 ). There was no significant heterogeneity between these studies $\left(I^{2} 0.0 \%, p=0.97\right)$. Two studies investigating SS-WBV reported small effect sizes for static balance (SMD $-0.26,95 \% \mathrm{Cl}-0.78$ to 0.25$)$, with no significant heterogeneity $\left(1^{2} 0.0 \%\right.$, $p=0.691)$. For change values two studies reported moderate effect sizes (SMD $-0.70,95 \% \mathrm{Cl}$ -1.13 to 0.28$)$ and no significant heterogeneity $\left(I^{2} 0.0 \%, p=0.519\right)$.

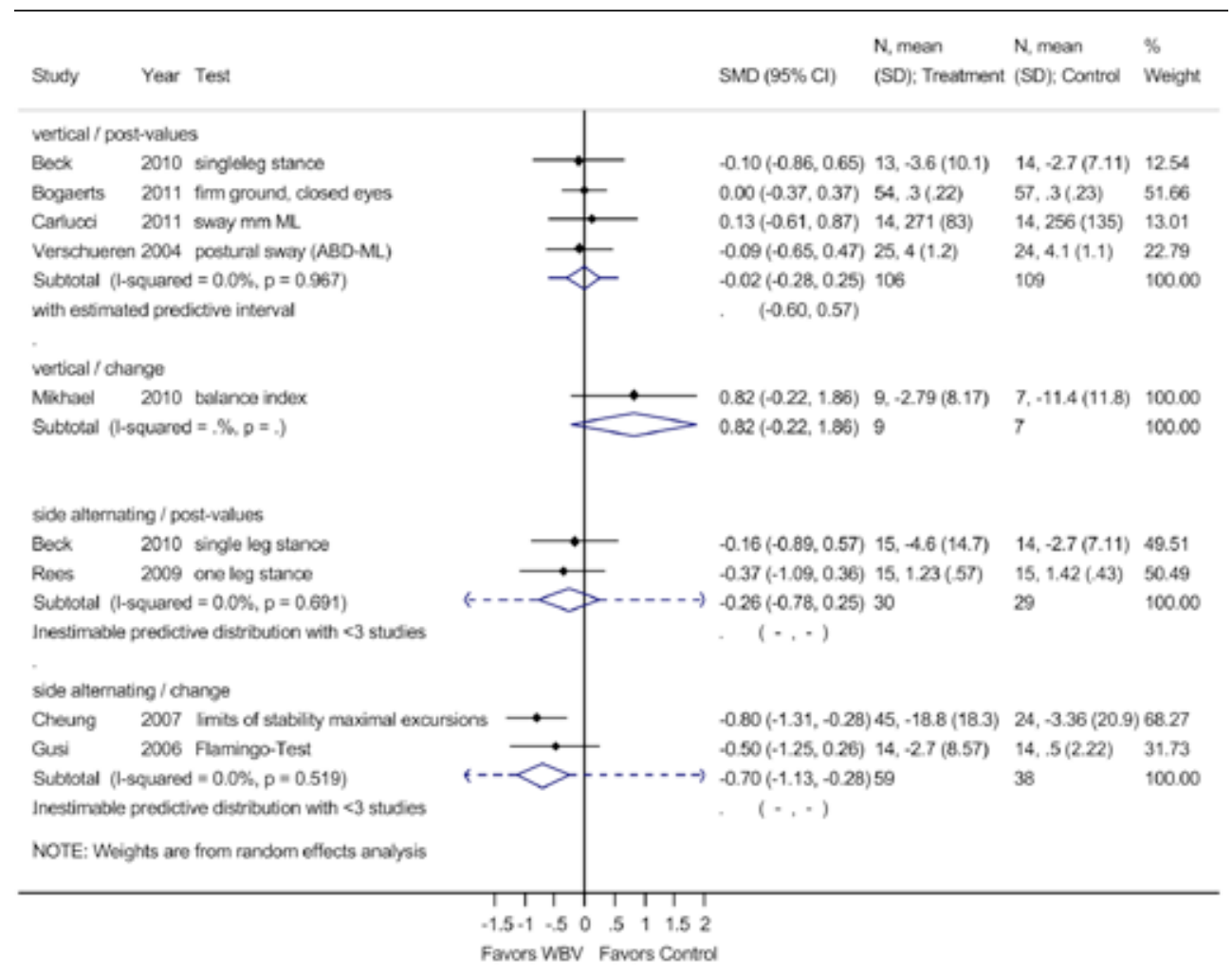

\section{Figure 3}

Forest plot of 8 trials (9 comparisons comparing) stratified for the vibration type (vertical and side alternating). Outcomes were tests for static balance. The analyses were separated for trials reporting post-values (i.e. mean and SD from follow-up) and for trials that reported change values (i.e. mean and SD from the changes from baseline to follow-up). Random effects model with predictive interval. The predictive interval indicates the range within which we expect the effects of $95 \%$ of future studies. Values on $\mathrm{x}$-axis denote SMDs. 


\section{Dynamic balance}

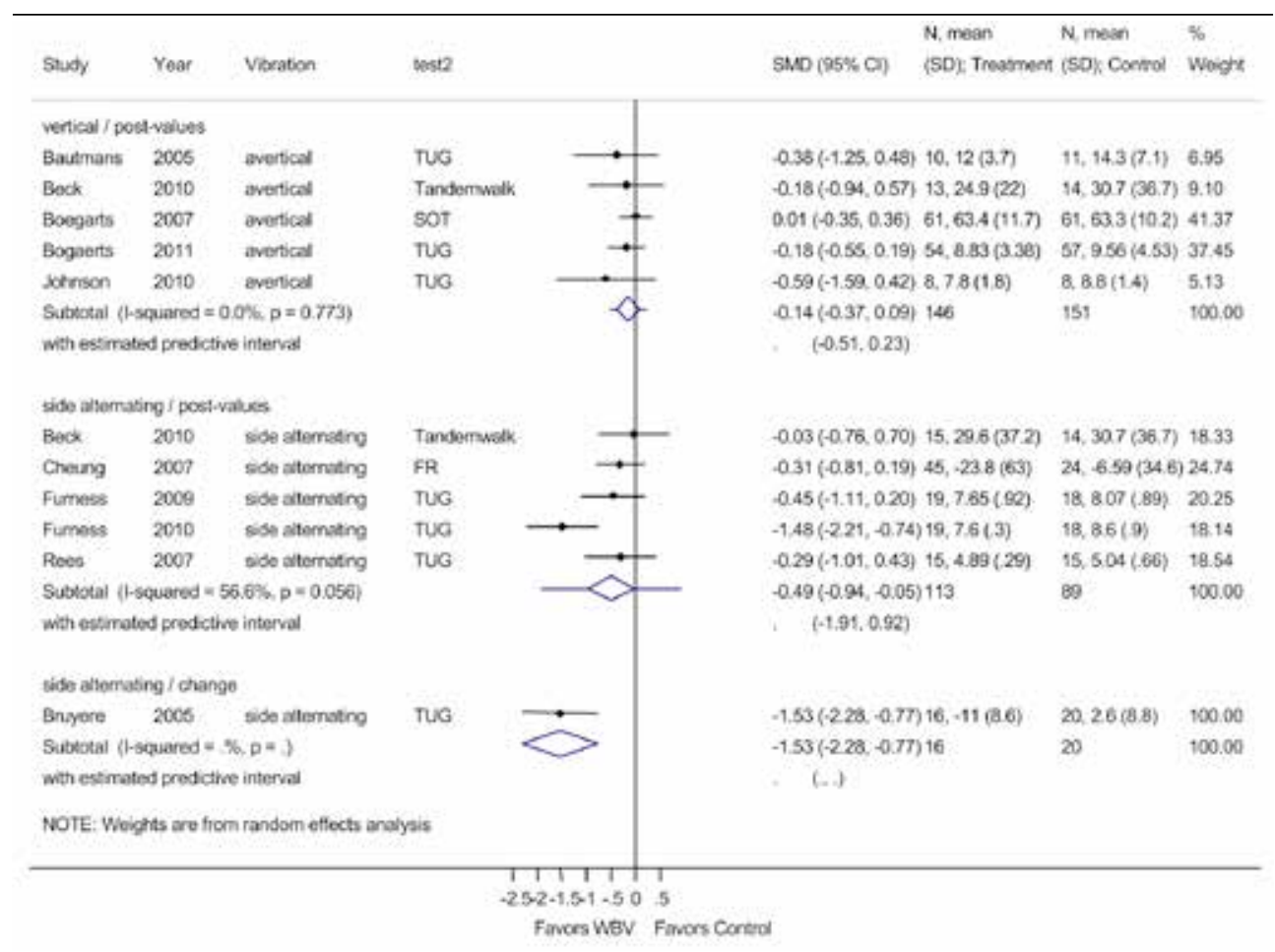

\section{Figure 4}

Forest plot of 9 trials comparing the effects of any type of vibration and control interventions on dynamic balance. The analyses were separated for trials reporting post-values (i.e. mean and SD from follow-up) and for trials that reported change values (i.e. mean and SD from the changes from baseline to follow-up). Random effects model with predictive interval. The predictive interval indicates the range within which we expect the effects of $95 \%$ of future studies. Values on $x$-axis denote SMDs.

For dynamic balance outcomes and VS-WBV post intervention mean values (additional file 3) five studies reported a small effect size (SMD $-0.014,95 \% \mathrm{Cl}-0.3$ to 0.17 ) favoring vibration training. No significant heterogeneity was found $\left(I^{2} 0.0 \%, p=0.48\right)$. In five papers reporting SS-WBV, a small to moderate effect size (SMD $-0.49,95 \% \mathrm{Cl}-0.94$ to -0.05 ) in favor of vibration training and evidence for middle heterogeneity $\left(I^{2} 56.6 \% ; p=0.056\right)$ was found.

Pooling of VS-WBV with SS-WBV in all studies reporting on TUG $(n=7)$ resulted in a weighted mean difference (WMD) of -0.86 , $(95 \% \mathrm{Cl}-1.61,-0.11)$. Considerable heterogeneity $\left(I^{2} 79.9 \% ; p\right.$ $=0.000$ ) was shown in this case (Figure 5). 


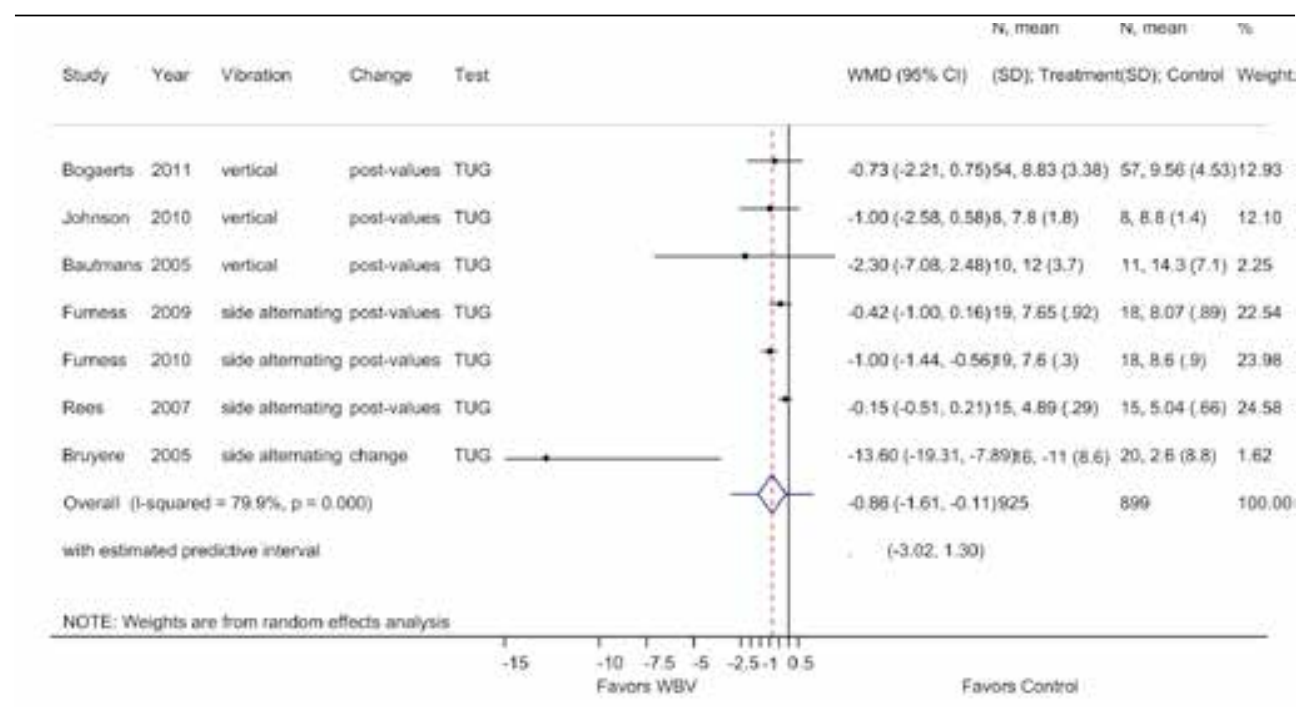

\section{Figure 5}

Forest plot of 7 trials comparing the effects of any type of vibration and control interventions on the Timed Up and Go Test. Random effects model with predictive interval. The predictive interval indicates the range within which we expect the effects of $95 \%$ of future studies. Values on $x$-axis denote WMD (weighted mean difference).

In an attempt to discriminate between WBV-plus-exercise-trials and WBV-without-exercise-trials we performed a subgroup analysis comparing overall effect sizes from studies reporting on dynamic balance with WBV combined with exercise versus WBV only (Figure 6). WBV-plusexercise revealed a small effect size of SMD -0.25 (95\% Cl -0.58 to 0.07) in dynamic balance. WBV-without-exercise revealed a moderate effect size of SMD -0.55 (95 \% Cl -1.09 to -0.01$)$. We also plotted a funnel plot (Figure 7) and performed an egger's test for small study bias (i.e. including the potential of publication bias). There was no evidence for small study bias neither in the funnel plot nor in the egger's test $(p=0.367)$. 


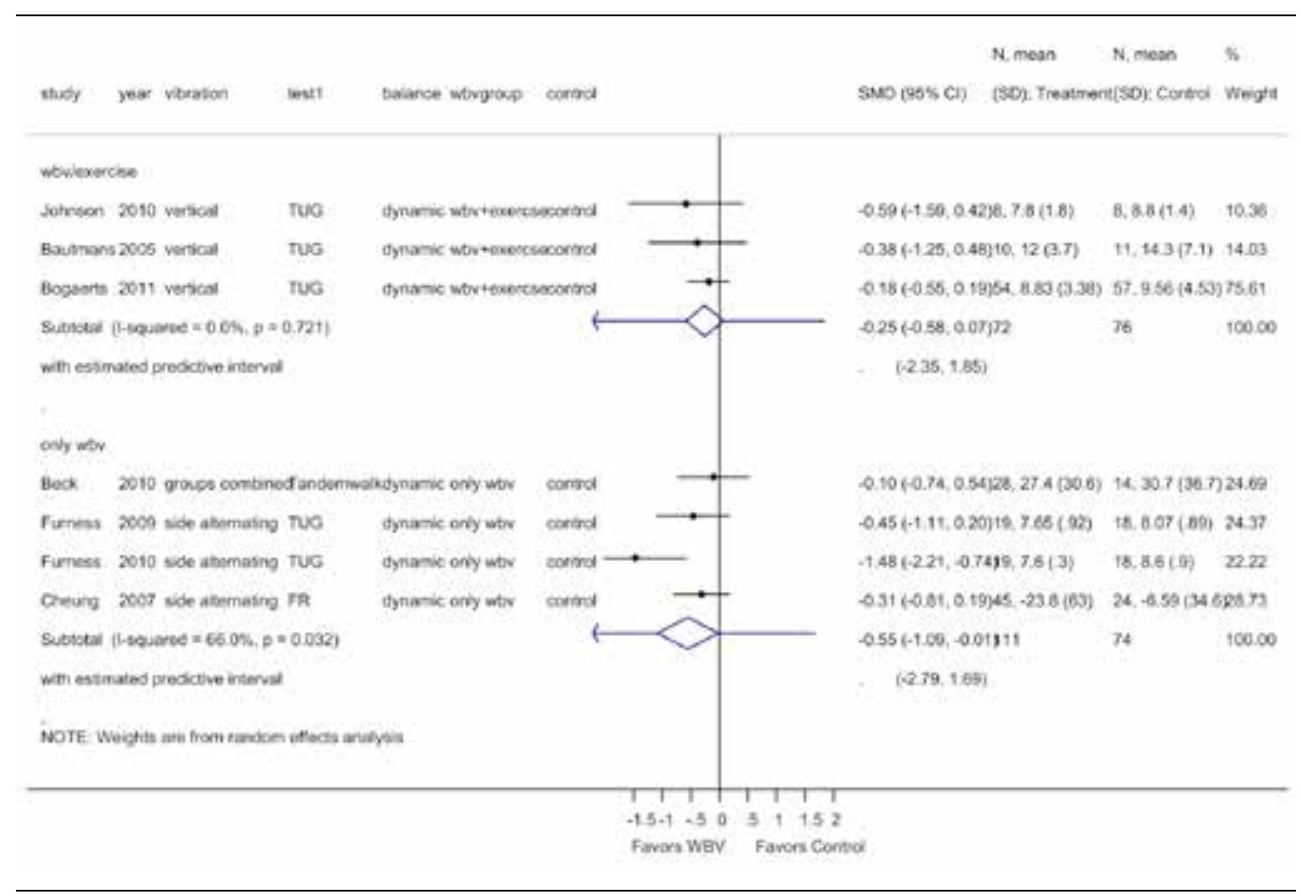

\section{Figure 6}

Forest plot of 6 trials comparing overall effects of WBV-plus-exercise versus isolated WBV. The analysis reports post intervention values. Values on $\mathrm{x}$-axis denote SMDs. 


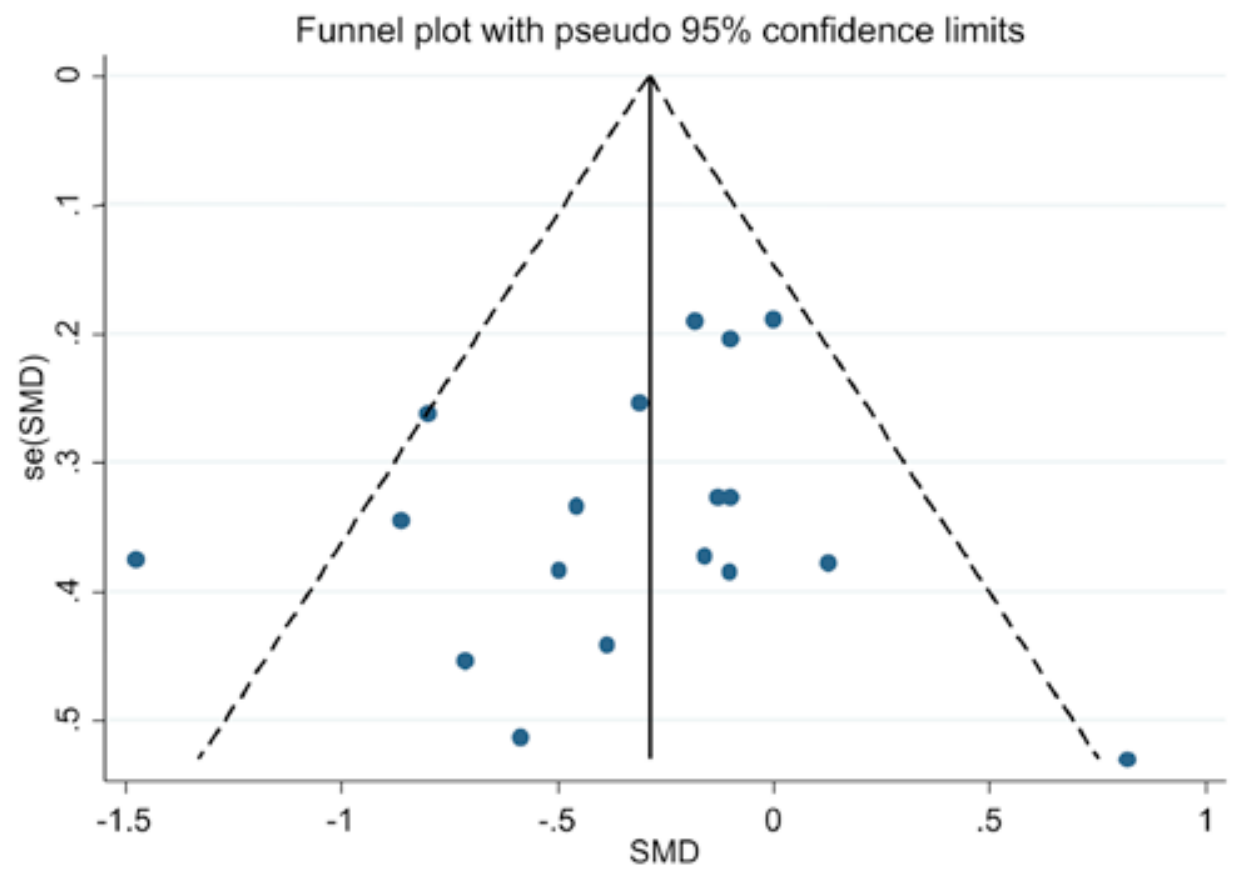

Figure 7

Funnel plot of estimated WBV treatments. A funnel plot with all points evenly distributed on both sides of the solid vertical line indicates no publication bias.

\section{Discussion}

This systematic review of 15 studies included a total of 933 participants. The main aims were to estimate the quality and internal validity of the studies and to describe the balance assessments used to assess the effect of WBV training. Furthermore, we aimed to provide some information about the clinical relevance of the studies. Following the results of the meta-analysis, it can be postulated that a side-alternating WBV (SS-WBV) intervention can improve dynamic balance in elderly subjects. This is based on the position of the diamond lying left of the 'no difference' line that indicates that WBV is beneficial. This finding is despite much variation in protocol design of the included studies and is, thus, encouraging that SS-WBV may be a viable precursor to more traditional forms of exercise training aiming to improving balance and function among sedentary and frail older adults. However, training using VS-WBV revealed only small effects for static (a) and dynamic balance (b), while SS-WBV showed small to moderate improvements for the same balance requirements. These findings seem to suggest that the vibration pattern of WBV platforms has differing influence on the postural outcomes. These differing findings might, however, also be a result of the still rather small amount of WBV studies available that were performed in elderly populations. This systematic review, therefore, only reveals first estimates 
for these measures and warrants further WBV research in larger populations. This in mind, the relationship between research with different WBV systems and their effect(s) on postural control in elderly individuals requires further exploration. Translating the results from WBV experiments with healthy elderly participants to therapeutic interventions should, therefore, take place with caution until the appropriate clinical studies with clinically relevant population outcomes have been conducted. For this reason, no clear recommendations for clinical use of WBV to improve dynamic balance in elderly can at present be made. These findings rather warrant further research in to the effects of SS-WBV on balance of elderly with sufficiently powered RCT study designs.

During WBV training that is combined with performance of active exercises theoretically two different stimuli for the muscle-nerve system can be identified and made responsible for the observed training effects: [1] reflex muscle contraction induced during vibration and [2] body weight exercises. This makes it difficult to attribute observed effects to the WBV training in those studies that use a combined training approach. However, the recently published meta-analysis of Steib et al. [56] showed that more traditional forms of resistance training have no effects on postural control. Our analysis where we separate the studies with WBV-plus-exercise-trials from WBV-without-exercise-trials shows that also SS-WBV without exercise shows a similar direction and magnitude of effect sizes and, therefore, indicates to have a positive influence on dynamic balance.

The frequency $(F)$, amplitude $(A)$, frequencies of training sessions per week (FRQ) of vibration stimuli either applied with SV-WBV or SS-WBV and their respective effect on balance outcomes reveals varying results. Low frequencies from 10 to $26 \mathrm{~Hz}$ showed higher effectiveness than frequencies between 30 to $40 \mathrm{~Hz}$. Most applications with frequencies between 10 to $30 \mathrm{~Hz}$ were performed on SS-WBV devices. The amplitudes vary from $0.5 \mathrm{~mm}$ to $8 \mathrm{~mm}$ in both types of vibration. The amplitudes seem not to have a significant role. Frequencies over $30 \mathrm{~Hz}$ generate a faster platform movement and deliver more energy to the body.

$F R Q$ describes the total time on the WBV device per duration of the vibration sessions, the total rest time between the duration of the vibration sessions and the sessions per week. The optimal time per workout with SS-WBV for static and dynamic balance appears to be: 3 vibration sessions of 60 seconds with 60 seconds rest time between each and a frequency of 3 times per week. For VS-WBV it is unclear which FRQ has to be used in order to attain optimal training results, because the different studies were applying different training parameters. For instance, it is unclear whether shorter sessions ( 3 sets of 15 seconds) or longer sessions ( 15 sets of 60 seconds) or sessions performed without a break lasting 15 minutes [52] are most effective. Future research must examine these aspects for VS-WBV. In addition, it is unclear whether loading parameters of $0.3 \mathrm{~g}$ (low intensity) performed with VS-WBV are comparable in effect to loading parameters of $0.45 \mathrm{~g}$ to $0.8 \mathrm{~g}$ with SS-WBV which seems to have no effect on balance. However, during SS-WBV the loading parameter started with low-intensity and was increased to high-intensity WBV during the course of the training period, thus adding a form of progression to the training $[48,49]$. This is another aspect that should be focused on in future studies. 
A point of attention in future studies should be the inclusion of both men and women in WBV studies when postural control is of concern. Four studies with VS-WBV [19, 40, 42, 52] were conducted with women only. For SS-WBV two studies [22, 47] with women only were conducted. All other studies were conducted with women and men. This makes it difficult to generalize the findings of this review to both genders due to the relative low amount of studies with information on men. Another point of concern is related to the age of the participants. Only three studies include a sample of volunteers over the age of 75 years. The age of 75 years, however, seems to be a threshold point for problems with postural control. Especially over 75 years of age healthrelated causes gain greater importance as causes for falls. The fact that this age group was not studied in the majority of the included studies might hypothetically explain the lack of observed effects in some of the studies.

\section{Study limitations}

We developed and utilized a structured study protocol to guide our search strategy, study selection, extraction of data and statistical analysis. However, a number of possible limitations of this review should be noted. First, a publication bias may be present, as well as a language bias, given that we restricted our search to English and German language publications. Second, we included 15 randomized trials. The risk of bias for these trials shows a high risk of bias for allocation concealment and blinding. Finally, the interventions in these trials were of relatively short duration when we consider the time of training in individual sessions and heterogeneous in their design. A Cochrane review that investigated exercise for improving balance in older people [36] showed that in general successful programs offer exercise sessions from 15-20 minutes up to 70 minutes per session that are performed over periods spanning from 5 weeks up to 12 months [36]. Compared to this many WBV studies offer exercise sessions that are rather short in duration.

Another item that should be critically viewed is the underlying assumption that postural balance measures are related to future falls in elderly populations. Falling is a complex phenomenon and, among elderly people, both intrinsic and extrinsic risk factors must be evaluated. Poor balance is assumed to be one of the major risk factors for falls among the elderly. According to the results of a recent review there indeed seems to be a relation between force platform parameters (derived in laboratory circumstances) and future falls. However, this relationship has mainly been derived from cross-sectional study designs, and we cannot state that this is also a causal relation as implied in this systematic review. The number of prospective studies relating measures of postural control with falls is in fact relatively low and the variation in the ways the various postural control assessments; i.e., force platform measurements [57], have been carried out and how the results have been analyzed in each case makes it difficult to draw definitive conclusions. 


\section{Future research}

Despite these limitations, we believe that our review provides useful information regarding the effects of WBV interventions aimed at improving postural control in the elderly. It also provides some guidance about the components that should be explicitly considered in future interventions to enhance their effect on postural control. Future studies evaluating the effects of WBV interventions should preferably involve RCT type studies carried out among diverse subpopulations of the elderly. Primary outcomes for such RCT's should, as previously suggested, include both physical activity and detailed evaluation of postural control related health outcomes assessed both in the short and longer term. Trials that ultimately intend to reduce the number of falls in their study population should preferably adopt the Prevention of Falls Network Europe (ProFaNE) recommendations. ProFaNE developed a common set of outcome definitions and measures for such future trials. One of the recommendations is to explicitly focus on falls as a primary outcome measure [58].

Based on the results of this review it can be assumed that especially SS-WBV has the potential to provide a small but significant benefit for postural control. It might be that the use of WBV in physical therapy holds promise for those patients or older deconditioned individuals that need to be "skilled-up" for regular training. These are for example frail elderly who cannot perform regular types of exercises, e.g. strength and balance training.

Furthermore, falls have multi-factorial causes [34]. The maintenance of balance depends on the interaction of multiple sensory systems, the motor, and integrative body system. A marked deficit in one of these factors may be sufficient to increase the risk of falling; however, a combination of minor or moderate impairments in multiple physiological domains may also increase the risk of falling. The significant improvement of one factor may be sufficient to decrease the risk of falling.

In addition, we deal with the risk factors of falls. Assessments are used to quantify the risk of falling. In these assessments, certain limit cut-off values are described. If the patient's balance capacity is below these cut-off values, the risk of falling is increased. Patients, who are slightly above the cut-off values, are only marginally able to improve postural control with WBV, but sufficiently for improvements in daily life.

This review does not allow formulating "best evidence" guidelines for WBV training to improve balance in elderly. However, the review indicates that there are different effects observable between purely vertically and side-alternating WBV systems. The observed effect sizes for these two systems seem to indicate that rather SS-WBV might have potential to influence postural control in elderly. This also because our findings seem to be supported by other reviews on this topic that included studies of albeit lower methodological quality $[27,59]$. 


\section{Conclusion}

Two kinds of sinusoidal WBV can be identified for vibration treatments or training sessions that aim to improve balance in elderly. The footplates vibrate either exclusively vertically or in a seesawing manner around a central axis [30]. The question about the effectiveness of this treatment modality cannot be answered conclusively because of several methodological shortcomings and the lack of statistical significance in several outcomes. Therefore, studies with sound methodology and an adequate number of elderly (deconditioned or frail) participants are needed.

\section{Competing interest}

The authors declare that they have no competing interests.

\section{Authors contribution}

$\mathrm{LR}$ initiated the idea for the meta-analysis. $\mathrm{KH}, \mathrm{RH}$, SR collected the data. $\mathrm{RH}$ conducted the statistical analyses. SR and EDB wrote the paper. KH helped to write the text. LR and EDB supervised the data collection, statistical analysis, and writing of the paper and critically revised the text for its content. All authors have read and approved the final manuscript.

\section{Acknowledgement}

We thank the Bern University of Applied Sciences for the support. 


\section{References}

1. Moreland J, Richardson J, Chan DH, O'Neill J, Bellissimo A, Grum RM, Shanks $\mathrm{L}$ : Evidence-based guidelines for the secondary prevention of falls in older adults. Gerontology 2003, 49(2):93-116.

2. Tinetti $M$, Mendes de Leon C, Doucette J, Baker D: Fear of falling and fall-related efficacy in relationship to function among community-living elders. J Gerontol 1994, 49(3):140-147.

3. Muir SW, Berg K, Chesworth B, Klar N, Speechley M: Quantifying the magnitude of risk for balance impairment on falls in community-dwelling older adults: a systematic review and meta-analysis. J Clin Epidemiol, 63(4):389-406.

4. Sturnieks DL, St George R, Lord SR: Balance disorders in the elderly. Neurophysiol Clin 2008, 38(6):467-478.

5. Tinetti M, Baker D, MCAvay G, Claus E, Garrett P, Gottschalk M, Koch M, Trainor K, Horwitz R: A Multifactorial Intervention to Reduce the Risk of Falling among Elderly People Living in the Community. $\mathrm{N}$ Engl J Med 1994, 331(13):821-827.

6. Hausdorff JM, Edelberg HK, Mitchell SL, Goldberger AL, Wei JY: Increased gait unsteadiness in community-dwelling elderly fallers. Arch Phys Med Rehabil 1997, 78(3):278-283.

7. Ebenbichler GR, Kerschan-Schindl K: Sicher Bewegen im Alter: Veränderungen der sensomotorischen Fähigkeiten. Phys Med Rehab Kuror 2009, 19:43-58.

8. Schutzer KA, Graves BS: Barriers and motivations to exercise in older adults. Prev Med 2004, 39(5):1056-1061.

9. Herren K, Rogan S, Hilfiker R, Radlinger L:
Vibrationen mit therapeutischen Effekten. PhysioActive 2009:39-44.

10. Rittweger J: Vibration as an exercise modality: how it may work, and what its potential might be. Eur J Appl Physiol 2010, 108(5):877-904.

11. Moezy A, Olyaei G, Hadian M, Razi M, Faghihzadeh S: A comparative study of whole body vibration training and conventional training on knee proprioception and postural stability after anterior cruciate ligament reconstruction. Br J Sports Med 2008, 42(5):373-378.

12. Wegner R, Ziaja C, J.P.W. W, Baur C: Leistungsoptimierung im Rahmen eines 8-wöchigen Vibrationstrainings und der Einsatz eines Kohörenzmessgerätes im Leistungssport. Leistungsport 2009, 6:2224.

13. Torvinen S, Kannu P, Sievanen H, Jarvinen TA, Pasanen M, Kontulainen S, Jarvinen TL, Jarvinen M, Oja P, Vuori I: Effect of a vibration exposure on muscular performance and body balance. Randomized cross-over study. Clin Physiol Funct Imaging 2002, 22(2):145-152.

14. Schuhfried O, Mittermaier C, Jovanovic T, Pieber K, Paternostro-Sluga T: Effects of whole-body vibration in patients with multiple sclerosis: a pilot study. Clin Rehabil 2005, 19(8):834-842.

15. Haas CT, Turbanski S, Schmidtbleicher D: Zum Einsatz randomisierter Oszillationen in der Prävention und Rehabilitation von Rupturen des vorderen Kreuzbandes im alpinen Skirennlauf. In. Frankfurt: Johann Wolfgang Goethe-Universität, Institute Sports Sciences; 2002. 
16. Haas CT, Buhlmann A, Turbanski S, Schmidtbleicher D: Proprioceptive and sensorimotor performance in Parkinson's disease. Res Sports Med 2006, 14(4):273287.

17. Mikhael M, Orr R, Fiatarone Singh MA: The effect of whole body vibration exposure on muscle or bone morphology and function in older adults: a systematic review of the literature. Maturitas 2010, 66(2):150-157.

18. Verschueren SM, Roelants M, Delecluse C, Swinnen S, Vanderschueren D, Boonen S: Effect of 6-month whole body vibration training on hip density, muscle strength, and postural control in postmenopausal women: a randomized controlled pilot study. J Bone Miner Res 2004, 19(3):352359.

19. Zha DS, Zhu QA, Pei WW, Zheng JC, Wu SH, Xu ZX, Li T, Chen JT: Does whole-body vibration with alternative tilting increase bone mineral density and change bone metabolism in senior people ? Aging Clin Exp Res 2011.

20. von Stengel S, Kemmler W, Engelke K, Kalender WA: Effects of whole body vibration on bone mineral density and falls: results of the randomized controlled ELVIS study with postmenopausal women. Osteoporos Int 2011, 22(1):317-325.

21. Gusi N, Raimundo A, Leal A: Low-frequency vibratory exercise reduces the risk of bone fracture more than walking: a randomized controlled trial. BMC Musculoskelet Disord 2006, 7:92.

22. Turbanski S, Haas CT, Schmidtbleicher D, Friedrich A, Duisberg P: Effects of random whole-body vibration on postural control in Parkinson's disease. Res Sports Med 2005, 13(3):243-256.
23. Haas CT, Turbanski S, Kessler K, Schmidtbleicher D: The effects of random whole-body-vibration on motor symptoms in Parkinson's disease. NeuroRehabilitation 2006, 21(1):29-36.

24. Torvinen S, Kannus P, Sievanen $H$, Jarvinen TA, Pasanen M, Kontulainen S, Jarvinen TL, Jarvinen M, Oja P, Vuori I: Effect of fourmonth vertical whole body vibration on performance and balance. Med Sci Sports Exerc 2002, 34(9):1523-1528.

25. Haas C, Schmidtbleicher D: Zu den Effekten mechanischer Schwingungen bei M. Parkinson. Rheuma aktuell 2002(3):810.

26. Merriman $\mathrm{H}$, Jackson $\mathrm{K}$ : The effect of wholebody vibration training in aging adults: a systematic review. Journal of Geriatric Physical Therapy 2009, 32(3):134-145.

27. Torvinen S, Kannus P, Sievanen H, Jarvinen TA, Pasanen M, Kontulainen S, Nenonen A, Jarvinen TL, Paakkala T, Jarvinen $M$ et al: Effect of 8-month vertical whole body vibration on bone, muscle performance, and body balance: a randomized controlled study. J Bone Miner Res 2003, 18(5):876884.

28. Torvinen $S$, Sievanen $H$, Jarvinen TA, Pasanen M, Kontulainen S, Kannus $P$ : Effect of 4-min vertical whole body vibration on muscle performance and body balance: a randomized cross-over study. Int J Sports Med 2002, 23(5):374-379.

29. Herren K, Rogan S, Hilfiker R, Radlinger L: Vibrationen mit therapeutisch interessanten Effekten. PHYSIOACTIVE 2009, 5:39-44.

30. Haas CT: Vibrationstraining, Biomechanische Stimulation und Stochastische Resonanz Therapie. pt Zeitschrift für Physiotherapeuten 2008, 
7(60):728-758.

31. Liberati A, Altman DG, Tetzlaff J, Mulrow C, Gotzsche PC, loannidis JP, Clarke M, Devereaux PJ, Kleijnen J, Moher D: The PRISMA statement for reporting systematic reviews and meta-analyses of studies that evaluate healthcare interventions: explanation and elaboration. BMJ 2009, 339:b2700.

32. Sackett D, Straus S, Richardson WS, Rosenberg W, Haynes RB (eds.): EvidenceBased Medicine, 2 edn. Edinburhg: Churchill Livingstone 2000

33. Orr R, Raymond J, Fiatarone Singh M: Efficacy of progressive resistance training on balance performance in older adults : a systematic review of randomized controlled trials. Sports Med 2008, 38(4):317-343.

34. Huxham FE, Goldie PA, Patla AE: Theoretical considerations in balance assessment. Aust J Physiother 2001, 47(2):89-100.

35. Howe TE, Rochester L, Jackson A, Banks PM, Blair VA: Exercise for improving balance in older people. Cochrane Database Syst Rev 2007(4):CD004963.

36. Turbanski S, Schmidtbleicher D: [Postural control depends on testing situation]. Sportverletz Sportschaden, 24(3):123-128.

37. Cohen J: Statistical Power Analysis for the Behavioral Sciences, 2nd edn. Hillsdale: Lawrence Erlbaum Associates; 1988.

38. Higgins J, Thompson S, Deeks J, Altman D: Measuring inconsistency in meta-analysis. BMJ 2003, 327:557-560.

39. Carlucci F, Mazza C, Cappozzo A: Does whole-body vibration training have acute residual effects on postural control ability of elderly women? J Strength Cond Res 2010, 24(12):3363-3368.

40. Bautmans I, Van Hees E, Lemper JC, Mets
T: The feasibility of Whole Body Vibration in institutionalised elderly persons and its influence on muscle performance, balance and mobility: a randomised controlled trial [ISRCTN62535013]. BMC Geriatr 2005 $5: 17$.

41. Bogaerts A, Delecluse C, Boonen S, Claessens $A L$, Milisen $K$, Verschueren SM: Changes in balance, functional performance and fall risk following whole body vibration training and vitamin $D$ supplementation in institutionalized elderly women. A 6 month randomized controlled trial. Gait Posture 2011, 33(3):466-472.

42. Bogaerts A, Verschueren S, Delecluse C, Claessens AL, Boonen S: Effects of whole body vibration training on postural control in older individuals: a 1 year randomized controlled trial. Gait Posture 2007, 26(2):309-316.

43. Johnson AW, Myrer JW, Hunter I, Feland JB, Hopkins JT, Draper DO, Eggett D: Wholebody vibration strengthening compared to traditional strengthening during physical therapy in individuals with total knee arthroplasty. Physiother Theory Pract 2010, 26(4):215-225

44. Mikhael M, Orr R, Amsen F, Greene D, Singh MA: Effect of standing posture during whole body vibration training on muscle morphology and function in older adults: a randomised controlled trial. BMC Geriatr 2010, 10:74.

45. Bruyere O, Wuidart MA, Di Palma E, Gourlay M, Ethgen O, Richy F, Reginster JY: Controlled whole body vibration to decrease fall risk and improve health-related quality of life of nursing home residents. Arch Phys Med Rehabil 2005, 86(2):303-307.

46. Cheung WH, Mok HW, Qin L, Sze PC, Lee 
KM, Leung KS: High-frequency wholebody vibration improves balancing ability in elderly women. Arch Phys Med Rehabil 2007, 88(7):852-857

47. Furness TP, Maschette WE: Influence of whole body vibration platform frequency on neuromuscular performance of communitydwelling older adults. J Strength Cond Res 2009, 23(5):1508-1513.

48. Furness TP, Maschette WE, Lorenzen C, Naughton GA, Williams MD: Efficacy of a whole-body vibration intervention on functional performance of communitydwelling older adults. J Altern Complement Med 2010, 16(7):795-797.

49. Rees S, Murphy A, Watsford M: Effects of vibration exercise on muscle performance and mobility in an older population. J Aging Phys Act 2007, 15(4):367-381.

50. Rees SS, Murphy AJ, Watsford ML: Effects of whole body vibration on postural steadiness in an older population. J Sci Med Sport 2009, 12(4):440-444.

51. Beck BR, Norling TL: The effect of 8 mos of twice-weekly low- or higher intensity whole body vibration on risk factors for postmenopausal hip fracture. Am J Phys Med Rehabil 2010, 89(12):997-1009.

52. Verschueren SM, Swinnen SP, Desloovere K, Duysens J: Vibration-induced changes in EMG during human locomotion. J Neurophysiol 2003, 89(3):1299-1307.

53. Steib S, Schoene D, Pfeifer K: Doseresponse relationship of resistance training in older adults: a meta-analysis. Med Sci Sports Exerc 2010, 42(5):902-914.

54. Piirtola $\mathrm{M}$, Era $\mathrm{P}$ : Force platform measurements as predictors of falls among older people - a review. Gerontology 2006, 52(1):1-16.
55. Lamb SE, Jorstad-Stein EC, Hauer K, Becker C: Development of a common outcome data set for fall injury prevention trials: the Prevention of Falls Network Europe consensus. J Am Geriatr Soc 2005, 53(9):1618-1622.

56. Madou KH, Cronin JB: The effects of whole body vibration on physical and physiological capability in special populations. Hong Kong Physiotherapy Journal 2008 -, 26:2438.

57. Rapp W, Boeer J, Albrich C, Heitkamp $\mathrm{H}-\mathrm{C}$ : Auswirkung eines Vibrations- und Krafttrainings auf die Beinmuskulatur bei Gonarthrosepatienten. Akt Rheumatol 2009, 34:240-245.

58. Zijlstra A, Ufkes T, Skelton DA, LundinOlsson L, Zijlstra W: Do dual tasks have an added value over single tasks for balance assessment in fall prevention programs? A mini-review. Gerontology 2008, 54(1):4049. 


\section{Additional material}

\section{Additional file 1}

Protocol review: Link: https://www.gesundheit.bfh.ch/fileadmin/wgs_upload/users/rgs2/ Protocol_systematic_review_2010_09_14..pdf

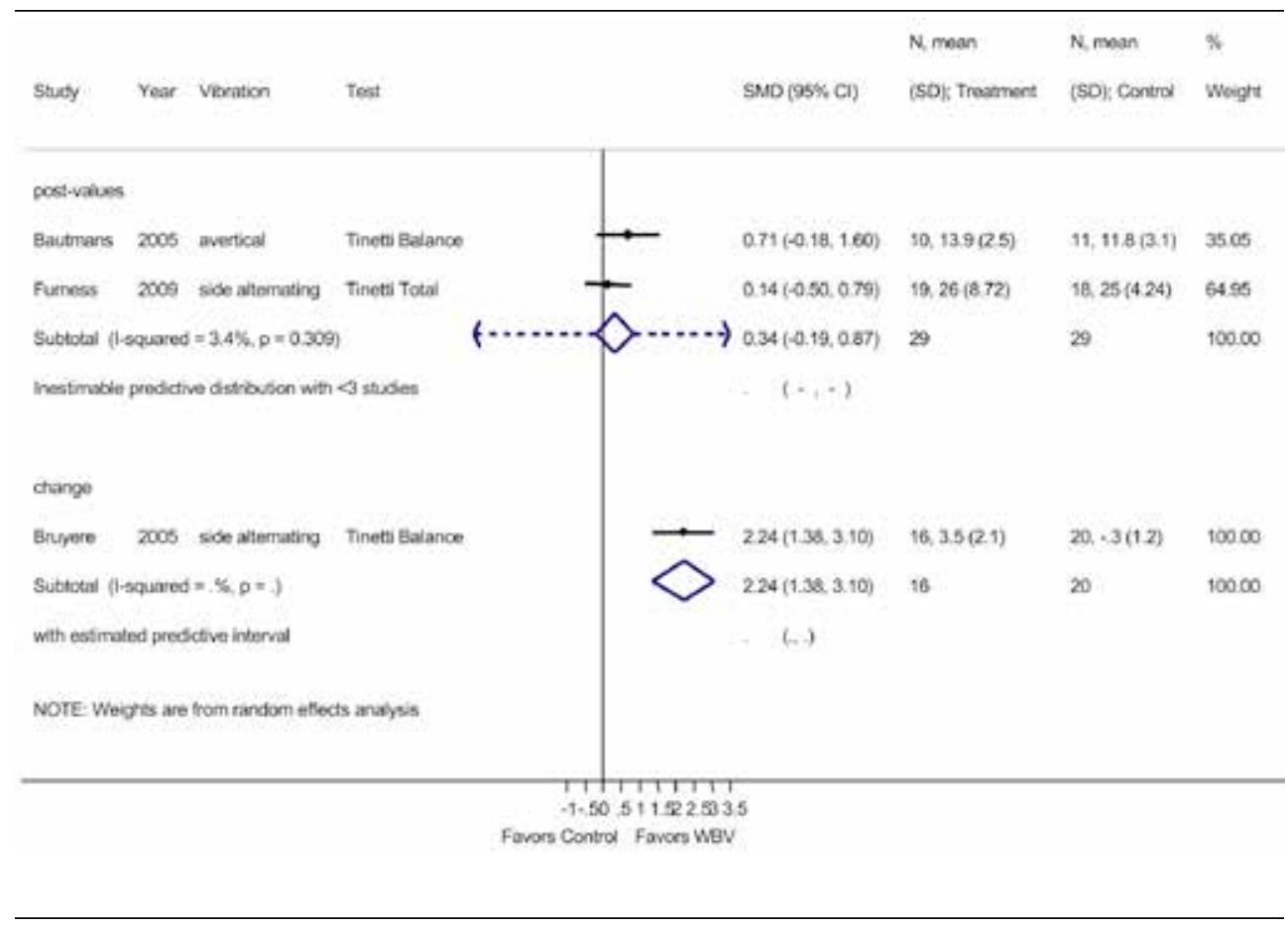

\section{Additional file 2}

Forest plot of 3 trials comparing the effects of any type of vibration and control interventions on mixed balance. The analyses were separated for trials reporting post-values (i.e. mean and SD from follow-up) and for trials that reported change values (i.e. mean and SD from the changes from baseline to follow-up). Random effects model with predictive interval. The predictive interval indicates the range within which we expect the effects of $95 \%$ of future studies. Values on $x$-axis denote SMDs 


\begin{tabular}{|c|c|c|c|c|c|c|c|c|c|}
\hline shady & Yeor & Weration & tesen & & & SMO (BOSC) & Iso: Tresativent & isok Cortroi & Weight \\
\hline \multicolumn{10}{|c|}{ 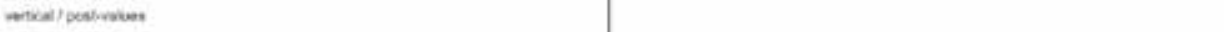 } \\
\hline Bactinass & 2005 & vences & TUO & & - & $-0.38,61.25,0.495$ & $12.12,37$ & $11.143(7.3)$ & 6.95 \\
\hline ted & 2010 & vertox & Tansimwar & & - & $-0.75(-0.9,0.57)$ & $13.24 .9(22)$ & $14,30 \times 08+1$ & 910 \\
\hline Bcosins & 2007 & venced & 801 & & - & $0.01(-0.35,0.36)$ & $61,63,4(11) 3)$ & $61,63.3\{90.2\}$ & 4139 \\
\hline Alogeen & 2011 & vertical & Tug & & & $-0.19(-0.55,0.19)$ & S4, ง & $57.956(453)$ & 3745 \\
\hline Johisean & 2010 & wertcal & too & & & $-0.50(-1.540 .02)$ & 2.7.8(1.8) & a. $\operatorname{ses}(94)$ & 5.13 \\
\hline \multicolumn{3}{|c|}{ Sutotal Bequared $=0.05, p=0.772)$} & & & & $-0.1+(-0.37,0.09)$ & 446 & 151 & 10000 \\
\hline \multicolumn{3}{|c|}{ Wat estimabe prodictive ntorva } & & & & - (-031, 62x) & & & \\
\hline \multicolumn{10}{|c|}{ 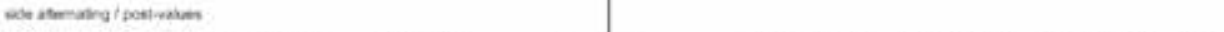 } \\
\hline bed & 2010 & Side atemakng & Tandenwas & & - & $-0.03(-0.70,0.70)$ & $48.2900(25)$ & 14,307007 & 18.53 \\
\hline Cheune & $200 t$ & vas atemarng & $\mathrm{rn}$ & & & $-0.21(-0$ à $1,0.1 \%$ & 45. $-23:$ (63) & $24-50$ CH 6 t & 2474 \\
\hline Fumese & 2005 & sos atemang & noo & & & $0.45(-1.51,020)$ & $+1,765(92)$ & $18.8 .60(85)$ & 20.25 \\
\hline Fumess & 2010 & vise atemuting & Tua & & & $-1,46(-2,21,-274)$ & $12.76(3)$ & $10.84(9)$ & 19.94 \\
\hline Heet & $200 t$ & sed aternarng & Tug & & - & $-020(-100,000)$ & $15.4 \mathrm{ka}(\mathrm{m})$ & 15. sos(6) & 184 \\
\hline \multicolumn{3}{|c|}{ Sutevtal (jeourot $=86.6 \mathrm{~S}, p=0.056)$} & & & & $-0.49(-0.94,0.05)$ & 113 & 82 & 10000 \\
\hline \multicolumn{3}{|c|}{ weh estmonod preactive nerve. } & & & & $+1.91,0.98)$ & & & \\
\hline \multicolumn{10}{|c|}{ 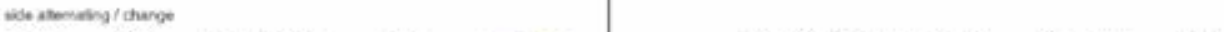 } \\
\hline Bnyer & 2005 & sube atimaking & tua & $\longrightarrow$ & & $-193(225,0 \mathrm{~m})$ & $46,-11(n e)$ & 20.28 (a) ai & 10000 \\
\hline \multicolumn{3}{|c|}{ 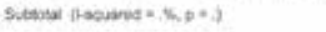 } & & & & $-1.93(-228,-0 m$ & 16 & 20 & 100,00 \\
\hline \multicolumn{3}{|c|}{ wh estimanod preactive ntarva } & & & & 1.3 & & & \\
\hline \multicolumn{10}{|c|}{ 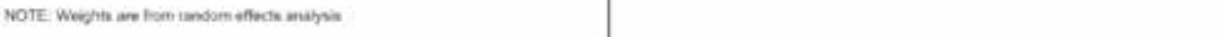 } \\
\hline \multicolumn{10}{|c|}{ 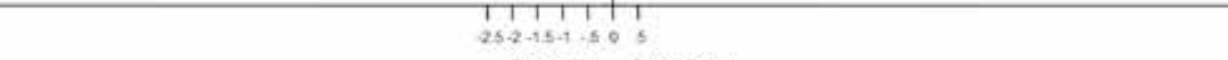 } \\
\hline & & & & Favon War & Fivon Contrit & & & & \\
\hline
\end{tabular}

\section{Additional file 3}

Forest plot of 11 trials (12 comparisons comparing) stratified for the vibration type (vertical and side alternating). Outcomes were tests for dynamic balance. The analyses were separated for trials reporting post-values (i.e. mean and SD from follow-up) and for trials that reported change values (i.e. mean and SD from the changes from baseline to follow-up). Random effects model with predictive interval. The predictive interval indicates the range within which we expect the effects of $95 \%$ of future studies will be. Values on x-axis denote SMDs. 



\section{Chapter 3}

Effects of whole-body vibration on proxies of muscle strength in old adults - a systematic review and meta-analysis on the role of physical capacity level

Rogan S

de Bruin ED

Radlinger $\mathrm{L}$

Jöhr C

Wyss C

Stuck N-J

de Bie RA

Hilfiker R 


\section{ABSTRACT}

\section{Background}

Dynapenia (age-associated loss of muscle strength not caused by neurologic or muscular diseases) and functional limitations (e.g. climbing stairs, chair rising) are important problems in elderly persons. Whole-body vibration, used as an adjunct to classical resistance training or even as a stand-alone alternative, might help to reduce these problems. Its value might be highest in elderly persons with very low function, where whole-body vibration can be used as a skilling up training until more conventional exercise types are possible. This systematic review and meta-analysis summarized the current evidence for whole-body vibration interventions on isometric maximum voluntary contraction, dynamic strength, power, rate of force development and functional strength in elderly categorised in different subgroups based on function levels.

\section{Methods}

An extensive literature search was carried out in February 2014 and repeated in February 2015 at PubMed, Cochrane Central Register of Controlled Trials, Physiotherapy Evidence Database and CINAHL electronic databases. The International Clinical Trials Registry Platform from the World Health Organization was also searched. Randomized controlled trials measuring isometric maximum voluntary contraction, dynamic strength, power, rate of force development and functional strength in studies using WBV intervention in 65 years or older elderly individuals were included. The methodological quality of included studies was assessed using the Cochrane Collaboration's tool for assessing Risk of Bias. Studies were classified based on the level of physical capacitiy of the participants as "Go-Go", "Slow-Go" or "No-Go". Data were pooled using a random effects model.

\section{Results}

38 articles of moderate methodological quality were included. The vibration modes for sinusoidal vertical whole-body vibration was between 25 to $40 \mathrm{~Hz}$, the amplitude varied from 2 to $4 \mathrm{~mm}$. Sinusoidal side-alternating whole-body vibration revealed frequencies from 2.5 to $35 \mathrm{~Hz}$ with amplitudes ranging from 0.05 to $12 \mathrm{~mm}$. Stochastic resonance whole-body vibration used frequencies between 3 to $6 \mathrm{~Hz}$. Effect sizes in Go-Go were moderate after vertical sinusoidal whole-body vibration compared to non-training control groups for isometric maximum voluntary contraction with effect size $0.48(95 \% \mathrm{Cl} 0.33$ to 0.63$)$ and for dynamic strength with effect size 0.47 (95\% Cl 0.06 to 0.88). Side-alternating sinusoidal whole-body vibration showed moderate effect sizes with $0.69(95 \% \mathrm{Cl} 0.32$ to 1.06$)$ for isometric maximum voluntary contraction, 0.50 (95\% Cl 0.07 to 0.92 ) for power, 0.40 (95\% $\mathrm{Cl} 0.16$ to 0.64 ) for rate of force development and 0.42 (95\% Cl 0.13 to 0.71 ) for functional strength compared to non-exercise control. No-Go showed for stochastic resonance whole-body vibration a moderate effect size with $0.50(95 \% \mathrm{Cl}-0.32$ to 1.33) for functional strength compared to non-exercise control. The effect sizes in the studies with participants in the No-Go group were generally higher compared to Go-Go and Slow-Go. 
The effective vibration forms for No-Goes are sidealternating sinusoidal whole-body vibration and stochastic resonance whole-body vibration.

\section{Conclusion}

Whole-body vibration shows beneficial effects, mainly in the No-Go group elderly compared to non-training control and convenional strength training groups. The results suggest that WBV can be used as a skilling-up exercise in participants not able to perform standard exercises.

Registration number: CRD42013006489. 


\section{Background}

Aging is associated with a decrease of muscle strength and power [1-3]. The term dynapenia, coined by Manini and Clark [3-5], best describes the condition of decreased muscle strength and power instead of the term sarcopenia. The latter only refers to an age-related loss in skeletal muscle mass. Muscle weakness is related to falls, lower walking speed, functional limitation, a decrease in mobility, and disability [6-8]. In this context, the elderly are viewed as a group of people in need [9]. When physical functioning is concerned there often is a mismatch between chronological age and biological age. Chronological age is not necessarily related to physical capabilities. For this rea- son, a classification of elderly based on physical abilities; e.g. physical and mental functions is more appropriate.

Zeyfang and Braun [10] classified older adults as "being an independent person" (Go-Go); "being a needy person with a slight handicap" (Slow-Go); and "being a person in need of care with severe functional limitation" (No-Go). The need for care may be defined as depending permanently on assistance (No-Go) or depending on support in everyday activities such as dressing, body care, eating, using the toilet, mobility, and planning the day (Slow-Go) [11].

The ability of elderly individuals to perform basic activities of daily life is crucial for their ability to exist independently [12]. To improve and/or enable performance of basic activities of daily life, exercise programs are indicated. Sensorimotor training and resistance exercises are effective methods to increase muscle mass and strength in the elderly [13]. Whole-body vibration (WBV) can be used as a sensorimotor training regimen. The impact of WBV on the body is low according to indicators such as blood pressure, heart rate, lactate, and O2 uptake [14-16]. Systematic reviews concluded that, compared to more demanding interventions, WBV might be a safer and less fatiguing type of exercise [17] with a beneficial effect on movement skills [18].

Three types of WBV are used based on the amount of vibrating plates $[18,19]$. Sinusoidal vertical whole-body vibration (SV-WBV) and sinusoidal side-alternating whole-body vibration (SS-WBV) use a single vibrating platform, whereas stochastic resonance whole-body vibration (SR-WBV) expects the trainees to stand on two platforms. During sinusoidal WBV the participants stand on a platform that vibrates vertically (SV-WBV) or to the side alternating (SS-WBV) with a high frequency be- tween 20 and $50 \mathrm{~Hz}$ and an amplitude between 2 and $14 \mathrm{~mm}$ [20]. SR-WBV vibrates with frequencies between 1 and $12 \mathrm{~Hz}$ and an amplitude between 3 and $6 \mathrm{~mm}$ while the feet of the participants are placed on two independent powered and stochastic vibrating platforms [무].

In recent years, WBV has been introduced as a training method to improve muscle power and

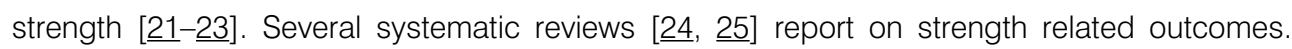
However, no review has considered muscle strength related outcomes in a comparison of WBV against non-exercising control or conventional exercise groups and no review evaluated the effects separately for groups differing in initial levels of physical functioning, e.g. the three groups 
"Go-Go, Slow-Go and No-Go". This is of relevance, however, since training principles would let us expect that those with the lowest level of fitness have greatest room for improvement. In other words, improvement in the outcome of interest will be greatest in those with lower initial values [26]. Furthermore, no re- view includes stochastic resonance WBV.

For clinicians, a systematic overview about the relevance and indication for application of SVWBV, SS- WBV or SR-WBV and how it might be applied for Go-Go, Slow-Go and No-Go elderly individuals is lacking. Therefore, the aims of this systematic review are to provide 1) an overview of the current studies on WBV, 2) to determine the effects of WBV on strength or power in Go-Go, Slow-Go and No-Go elderly individuals and 3) give recommendations on available evidence for practical use. We hypothesized that WBV differently effects on measures of strength and power in Go-Go, Slow-Go and No-Go.

\section{Methods}

\section{Data sources and searches}

Inclusion criteria and analysis methods were developed and documented in a protocol prior to the current review. Included were elderly over 65 years of age; excluded were Geriatric diseases (Parkinson disease, Stroke, Multiple sclerosis), studies applying electrical current vibration or vibration with shoe insoles. More detailed information on the protocol including a link to the search strategy can be found on http://www.crd.york.ac.uk/PROSPERO/display record.asp?ID=CRD42013006489 (PROSPERO registration number 2013:CRD42013006489). This systematic review and meta-analysis followed the PRISMA guidelines [27]. A first literature search of electronic databases was repeatedly carried out from January 2013 to February 2014 in the PubMed, CENTRAL (Cochrane Central Register of Controlled Trials), Physiotherapy Evidence Database (PEDro) and CINAHL electronic databases. The International Clinical Trials Registry Platform from the World Health Organization (WHO) was also searched. In February 2015 the search was repeated shortly before submission of the manuscript to ensure inclusion of most recent relevant material in the review. Additionally, a manual search of the reference lists of retrieved publications was conducted. English and German language restrictions were imposed upon the search.

\section{Systematic search}

The following keywords and combinations according to the PICO-model [28] were used in the search strategy:

Population: elderly, aged, dwelling home, nursing home, human research.

Intervention: Whole Body Vibration, WBV, noise, random vibration, RCT.

Comparator: WBV against control intervention

(non-exercise or exercise on a level too low to effect on muscle [29] ]), WBV against conventional 
strength training intervention.

Outcome: strength, maximal voluntary contraction, power, rate of force development, performance, falls. Our search terms are detailed in Additional file 1.

Based on the four PICO components, a final question was stated as: For an older adult with diminishing physical capacity, will whole body vibration (WBV) exercise as compared to non- or conventionally exercising older adults improve muscle strength and/or power?

The following aspects were operationalized: 1 ) assessment of the quality and internal validity of the studies reviewed; 2) description of the assessments used to document the effect of WBV on isometric maximal voluntary contraction (IMVC), dynamic strength (DS), power, rate of force development (RFD) and functional strength (FS); 3) composition of the WBV training parameters; and 4) conclusion about clinical relevance in general.

\section{Study selection}

Five independent reviewers (CJ, CW, NJS, SR, RH) screened the titles and abstracts for eligibility. They screened for randomized controlled trials (RCTs) measuring maximal voluntary contraction, power and rate of force development in studies using WBV intervention in elderly individuals (mean age at least 65 years). Full text articles in English or German were eligible for inclusion. Healthy elderly participants and all clinical outcome measures of IMVC, DS, power, RFD, and FS were included in this review. Studies describing vibrations applied by electrical current or vibrating insoles, and patient series were excluded.

In the event of missing data, additional information was requested from the corresponding authors in order to include these data in our meta-analysis.

\section{Data extraction}

In addition, general characteristics of the studies were extracted. Five authors (CJ, CW, NJS, $\mathrm{SR}, \mathrm{RH}$ ) independently abstracted the following information from each of the studies included in this review: 1) design and sample; 2) inclusion criteria; 3) training parameters (i. e. duration, frequency, intensity of WBV); 4) type of vibration plate; 5) change in strength, power, RFD; 6) conclusions of the studies and statistical significance.

\section{Methodological quality assessment of studies}

The methodological quality of the included articles was rated with the "Cochrane Collaboration tool for assessing risk of bias" (RoB) [을 to assess the risk of over- or under-estimating the effects of an intervention [31].

Nine items, with each having three rating categories, were scored and divided into six domains of bias (Fig. 2):

(1) low ROB, (2) unclear ROB and (3) high ROB. Rating

(1) is unlikely to alter the results significantly, (2) raises some doubt about the results and (3) seriously weakens confidence in the results. With insufficient information on an item, the score 
given was "high risk". The arbitration of a third reviewer was used in the event of any disagreement between the reviewers $(\mathrm{YB}, \mathrm{RH})$ for both ratings.

\section{Data synthesis and analysis}

Most outcomes of interest were presented as continuous data (mean values and SD or mean changes). For the meta-analysis of the present study the standardized mean difference (SMD) and $95 \%$ confidence interval $(\mathrm{Cls})$ of the post-intervention values or changes in scores were used for all comparisons. SMDs were pooled with a random effects model. The magnitude of the effect sizes for the between groups comparisons, calculated by SMDs are interpreted as follows: an effect size (d) around 0.2 indicates a small effect size, around 0.5 a medium effect size, and around 0.8 a large effect size [32]. If only one study was identified or data were not presented in a format that allowed inclusion in the data- set, results of individual studies are presented. If studies reported more than one IMVC, DS, power or RFD, and FS; then we only extracted the first outcome data on a hierarchy of outcomes:

IMVC: 1. isometric knee extension, 2. isometric hip extension, 3. isometric leg press; DS: 1. dynamic leg press, 2. dynamic knee extension (lowest speed), 3. dynamic hip extension; Power: 1. leg press, 2. knee extension; RFD: 1. counter movement jump, 2. squat jump, 3. leg press,; FS: 1. chair rise time, 2. chair rise repetition, 3. chair rise power, 4. stair climb, 5. wall squat. Subgroup analyses were undertaken to assess the effects of WBV on IMVC, DS, power, RFD and FS in Go-Go, Slow-Go and No-Go.

Heterogeneity was assessed by forest plots and the 12 statistics. Values $>25 \%$ indicate small, $>50 \%$ middle and $>75 \%$ considerable heterogeneity [33]. All other infor- mation was summarized and analysed qualitatively. Stata (version 13) was used for all meta-analyses.

\section{Results}

\section{Study selection}

The literature search yielded 1383 studies (PubMed $n=394$, Central $n=163$, Cinahl $=446$, Embase $=174$, Pedro $n=144$, ScienceDirect $n=29$, WHO International Clinical Trials Registry Platform $n=33$ ). After identification of 367 duplicates, 1016 titles and abstracts were screened. Seventy-nine studies remained for further full-text analysis. Subsequently, 41 studies were excluded because they did not address strength, power or rate of force development or included participants with diseases. Finally, 38 full text papers [20, 23,$\underline{34}-\underline{64}]$ were included for this review and 37 were used for the meta-analysis (Fig. 1). 


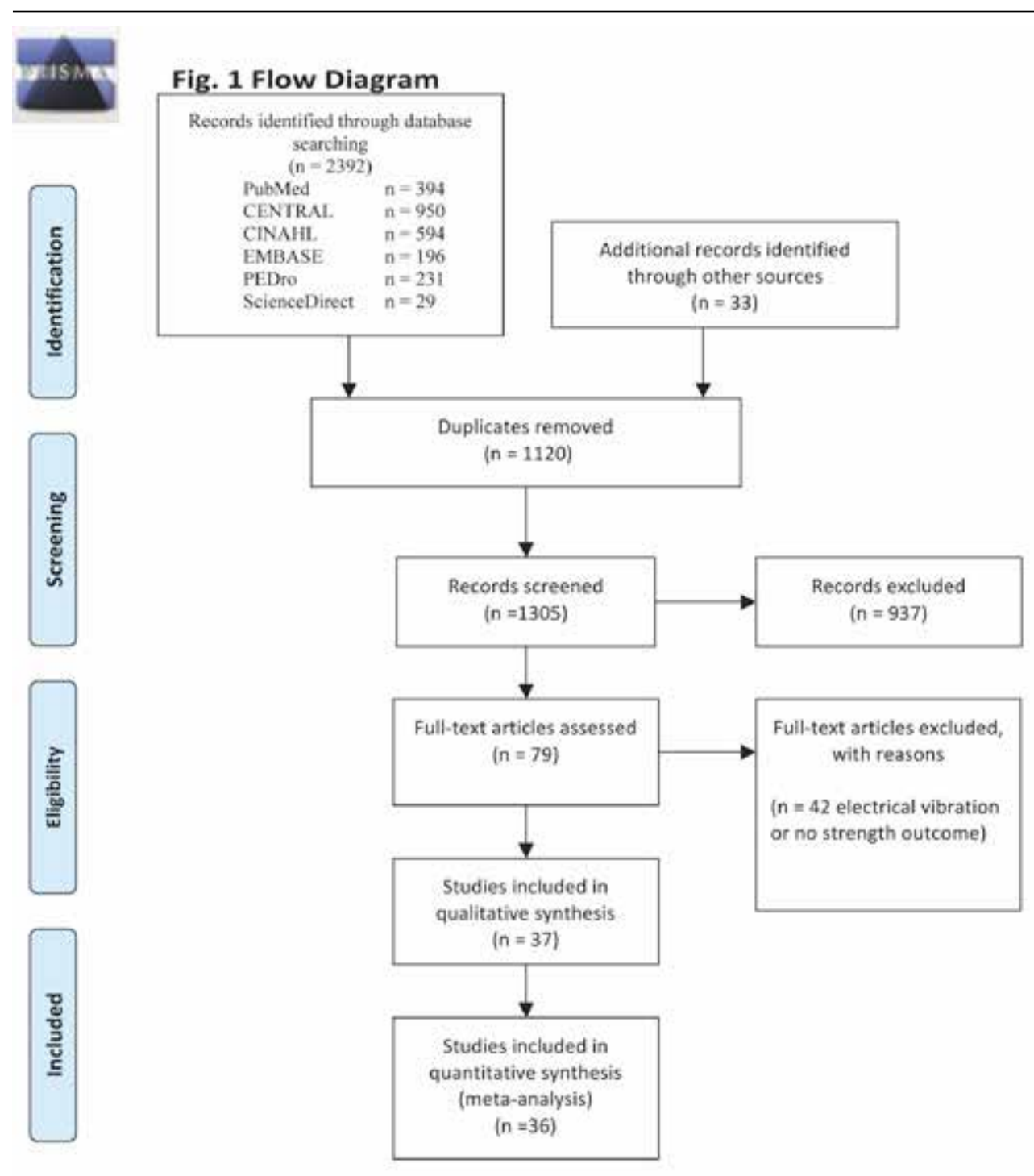

From: Moher D. Lberati A. Tetzlaff J. Alman DG. The PRISMA Group (2009). Pteferred Reporting fems for Systematic Reviews and Meta Analysen: The PRISMA Statement. PLOS Med E(6)) e1000097, doi: 10.1371/journal pmed 1000097

For more information, visit www.prisma-statement.org. 


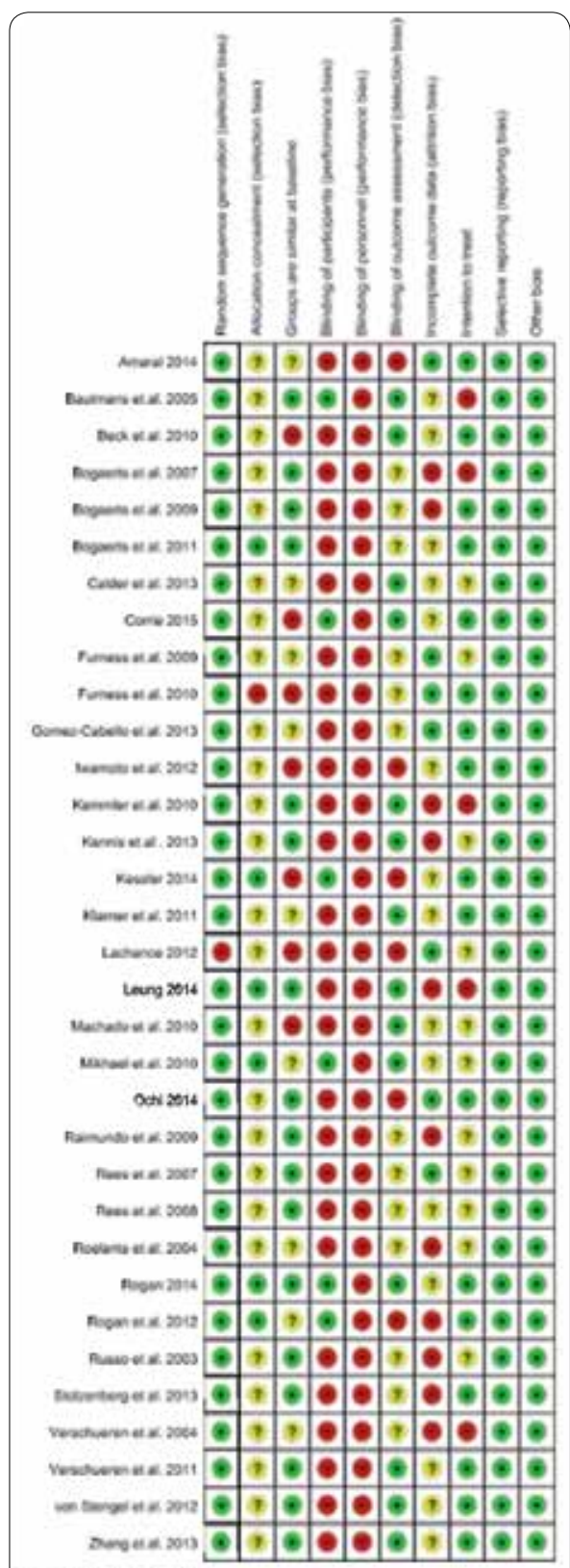

Fig. 2 Risk of bias 


\section{Study characteristics}

The major characteristics of the included studies are summarized in Table 1 . Three papers investigated the effects on force by vertical and side-alternating sinusoidal WBV $[35, \underline{46}, \underline{66}]$. Five studies included more than 100 participants. Leung et al. [66] $(n=596)$, Boegarts et al. [38] $(n=180)$, Kemmler et al. [44] $(n=151)$ von Stengel [59] $(n=151)$ and Sitjà-Rabert et al. [64] $(n=117)$. The other included studies had a small sample size of less than 100 participants. The used strength outcomes varied across all included studies.

Table $\underline{2}$ shows the training parameters. All authors prescribed two to three WBV sessions per week. Intervention duration of six trials were lasting more than 1 year $\underline{37}, \underline{38}, \underline{44}, \underline{46}, \underline{59} \underline{67}]$. The duration of ten trials $[35, \underline{36}, \underline{42}, \underline{43}, \underline{50}, \underline{55}-\underline{58}, \underline{53}]$ was between 6 months and 1 year. The other trials lasted less than 6 months while one study examined strength effects immediately after a single WBV intervention [20]. The training parameters such as amplitude, frequency and sets of WBV varied across all studies investigating sinusoidal WBV. In contrast, studies with SR-WBV were more homogenous.

\section{Study methodological quality}

All studies included in Fig. $\underline{2}$ were at risk of bias according to the "Cochrane Collaboration's tool for assessing risk of bias". Most studies lacked allocation concealment, blinding, and presented incomplete data. 


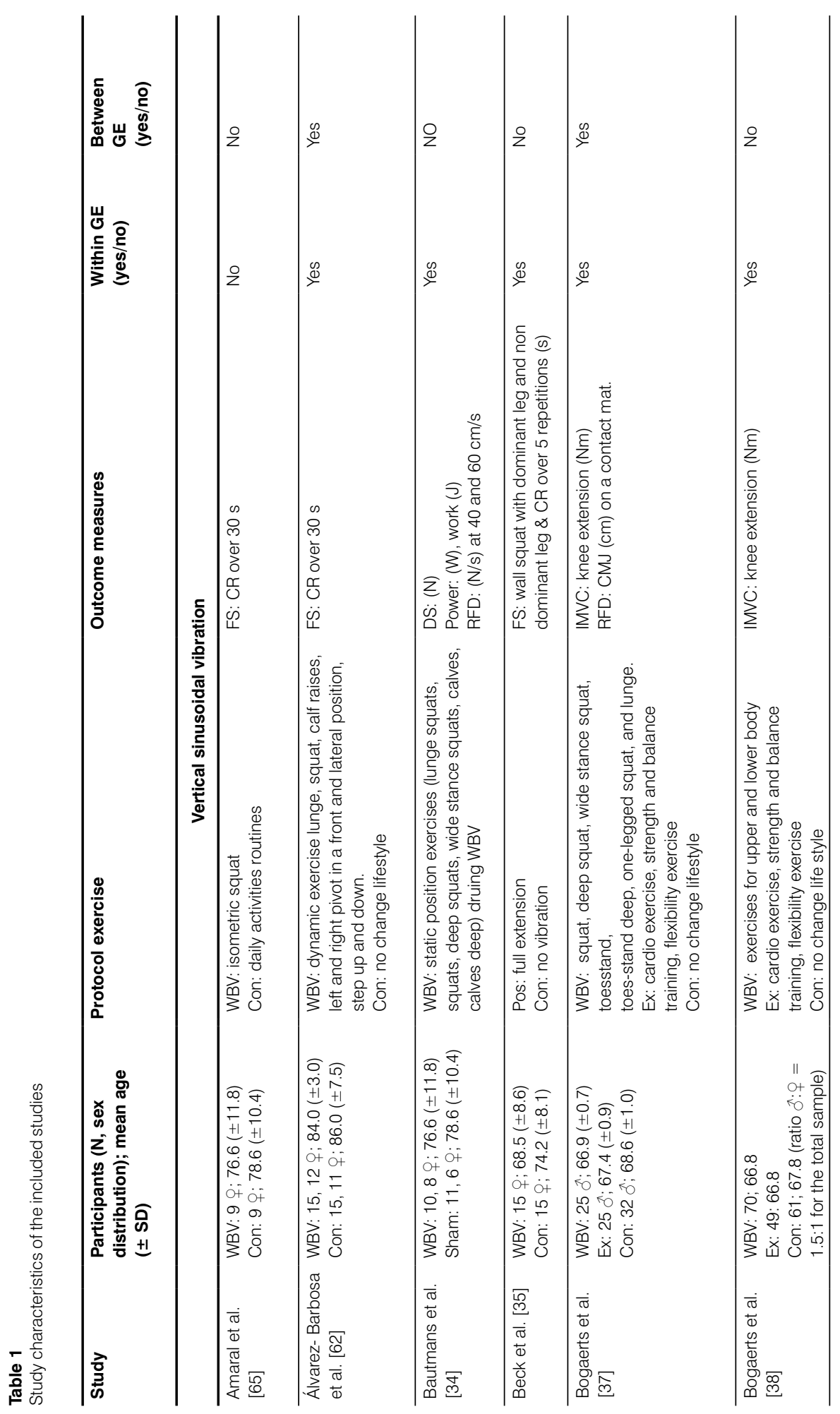




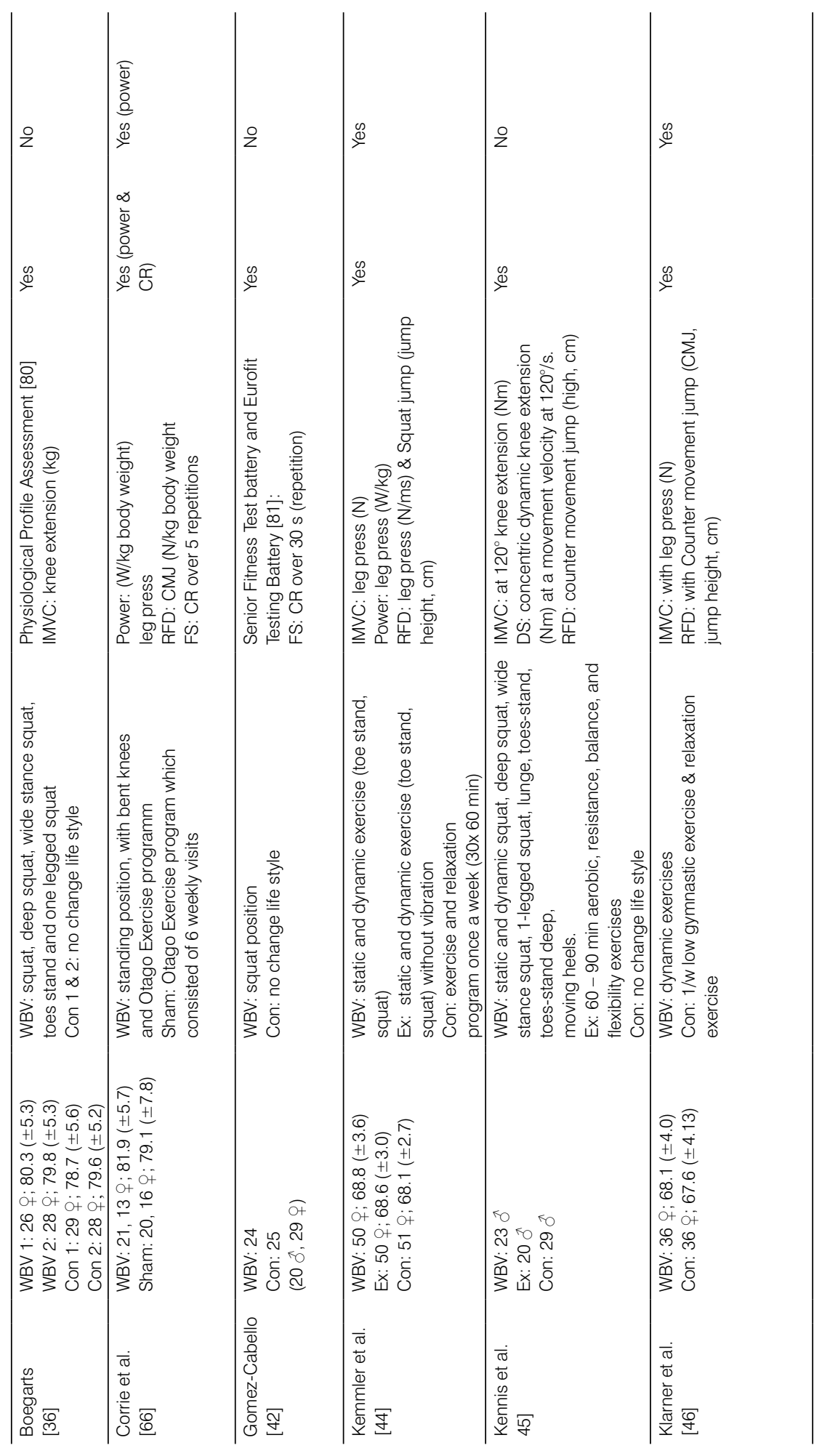




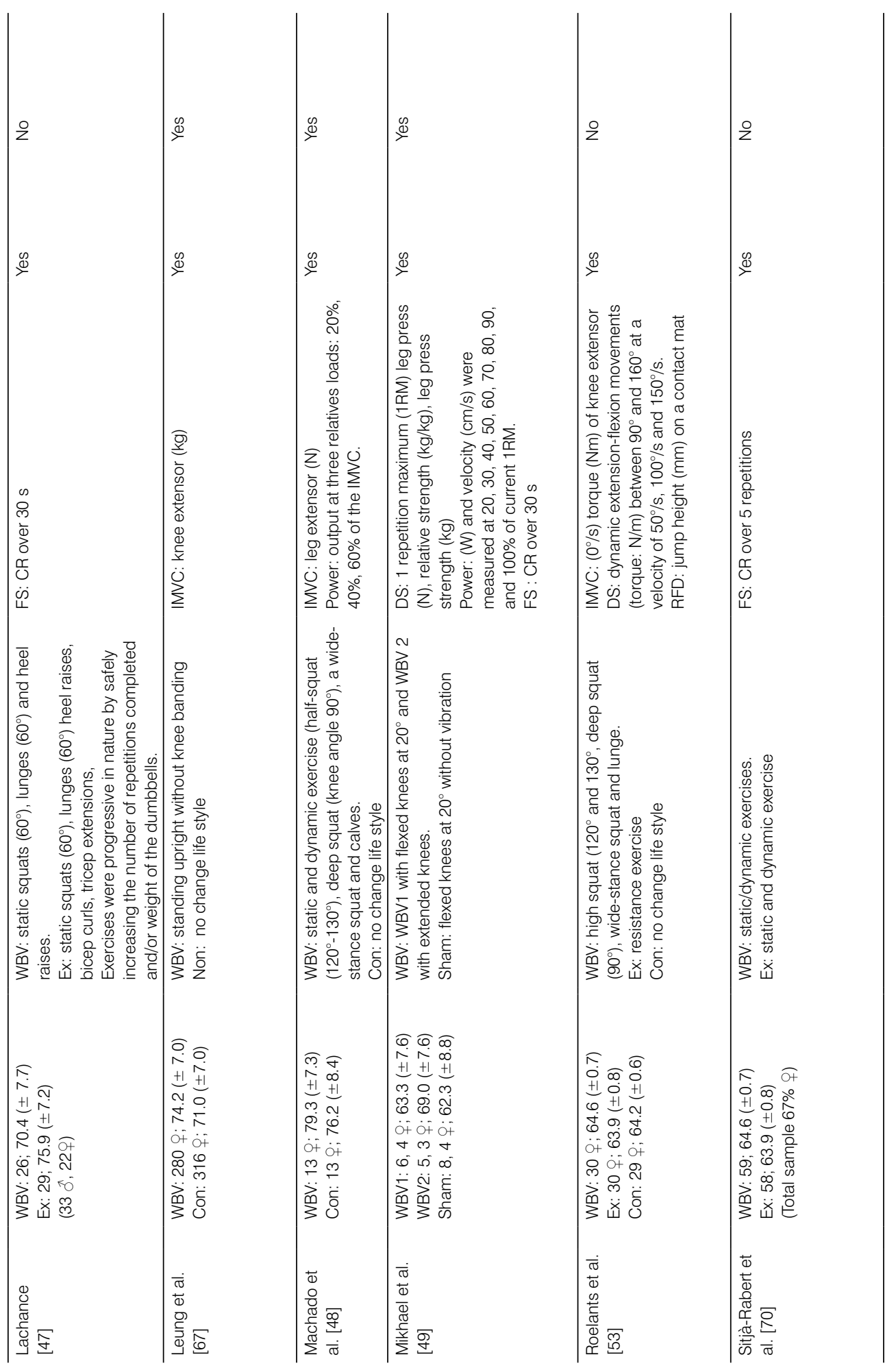




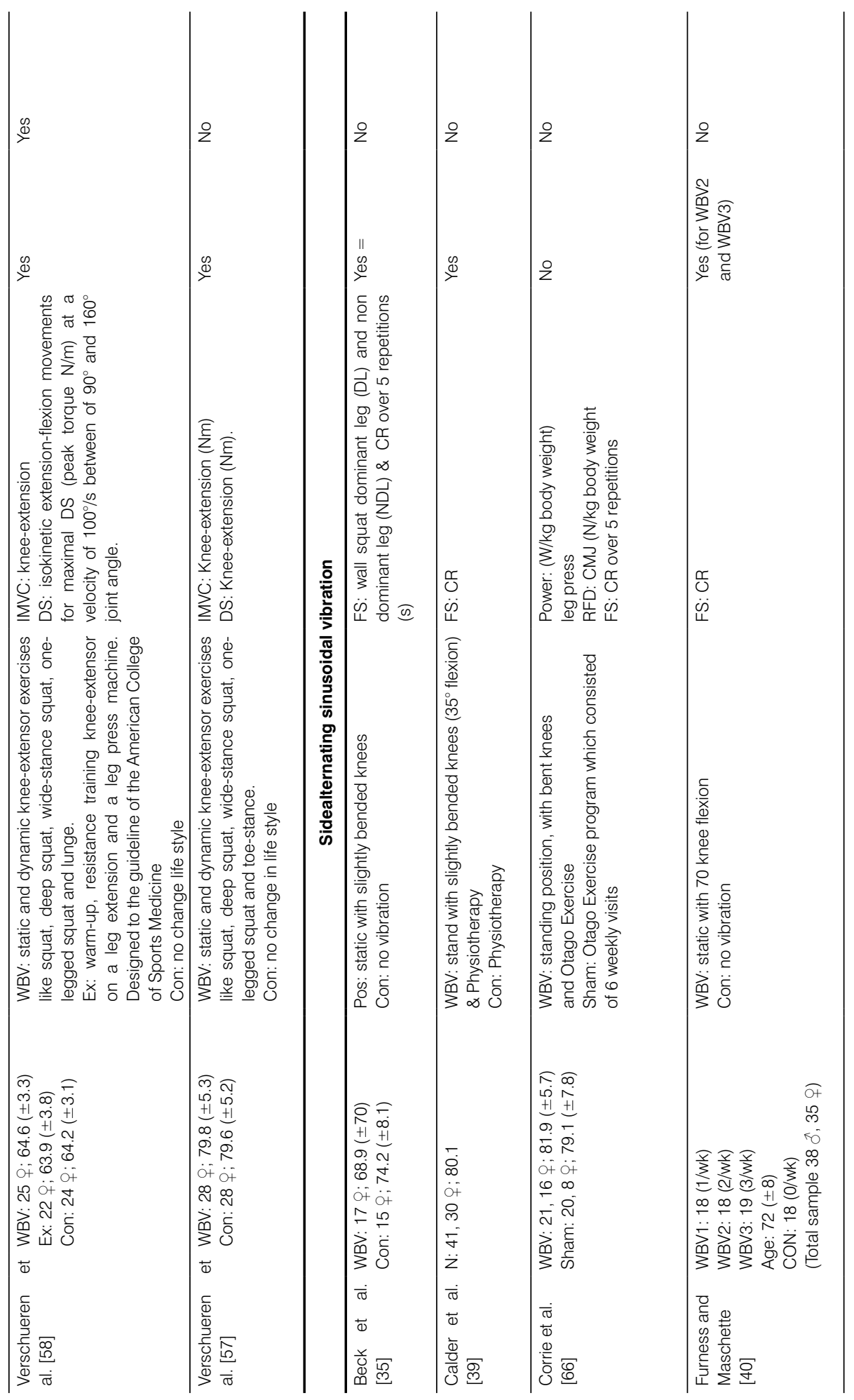




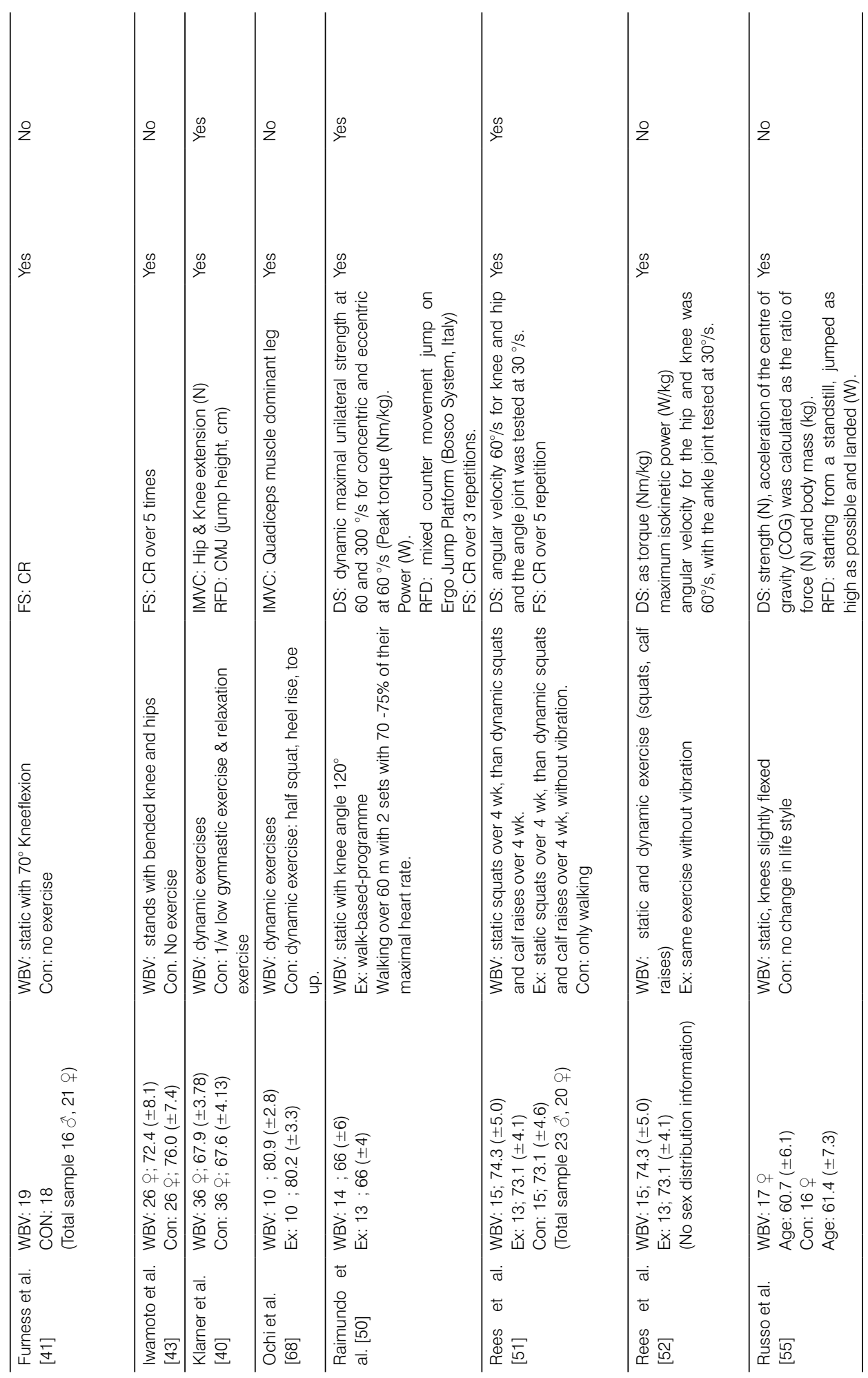




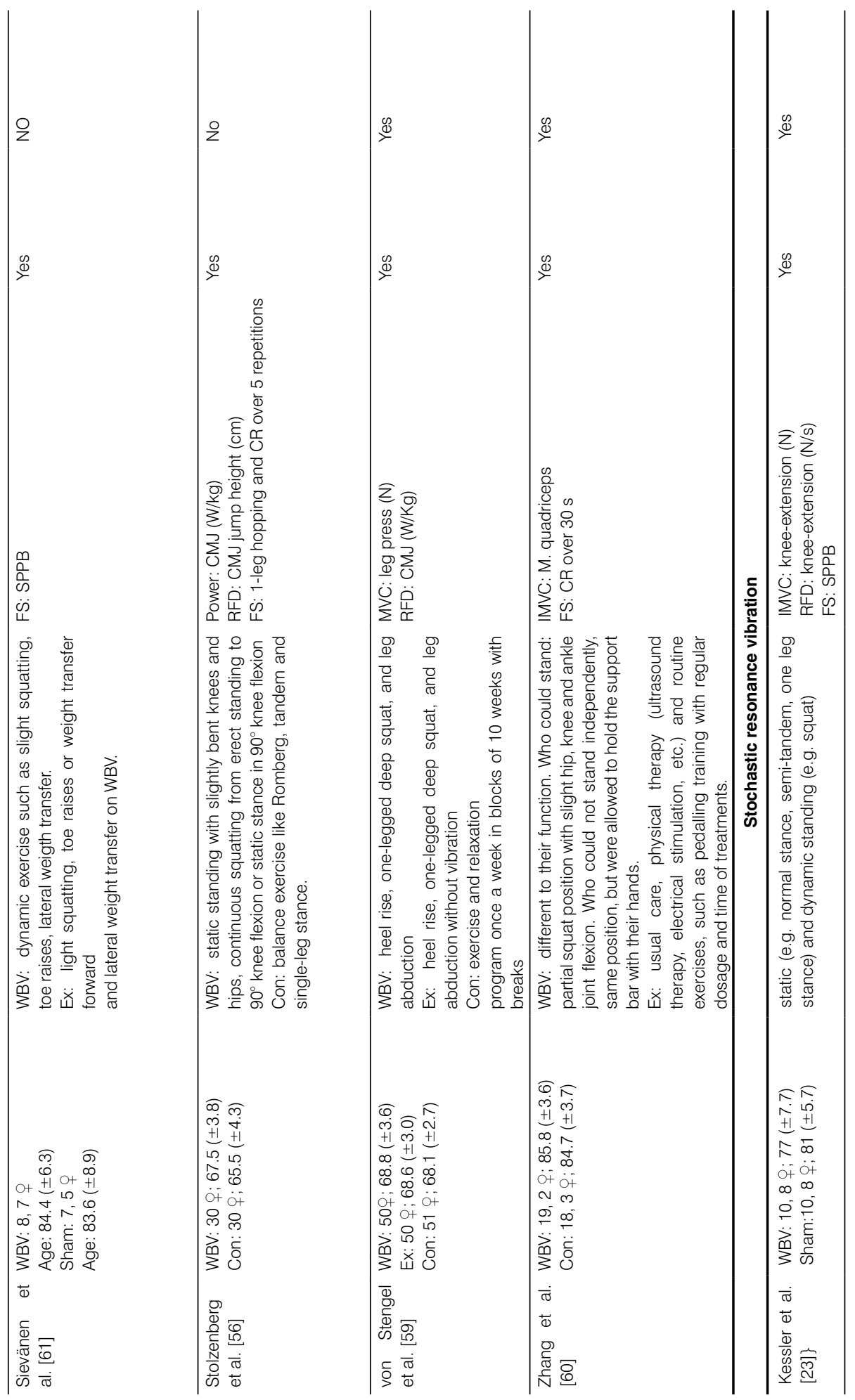




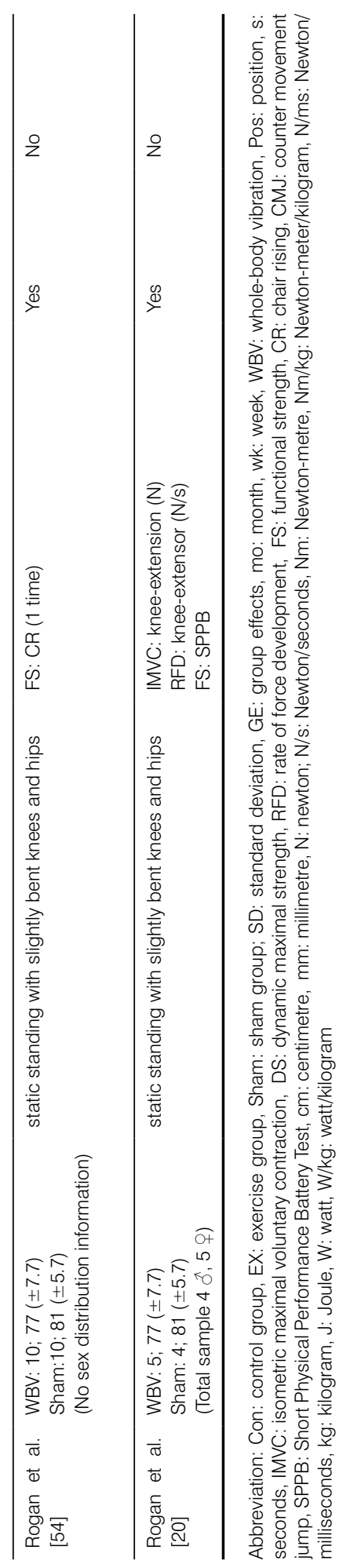


Table 2.

Overview Trainings parameter

\begin{tabular}{|c|c|c|c|c|}
\hline Study & $\begin{array}{l}\text { Duration/(Session/ } \\
\text { per week) }\end{array}$ & Amplitude & Frequency & $\begin{array}{l}\text { Sets, Duration, Rest between } \\
\text { set }\end{array}$ \\
\hline & \multicolumn{4}{|c|}{ Vertically sinusoidal vibration } \\
\hline Amaral etal. [78] & 12 weeks/(3/week) & $2-4 \mathrm{~mm}$ & $30-40 \mathrm{~Hz}$ & 3 sets $\times 30-45 \mathrm{~s}$ \\
\hline Álvarez-Barbosa et al. [62] & 8 weeks/(3/week) & $4 \mathrm{~mm}$ & $\mathrm{~F}: 30-35 \mathrm{~Hz}$ & $\begin{array}{l}6-12 \text { sets, } 12-17 \text { min total time, } 45 \mathrm{~s} \\
\text { rest between set }\end{array}$ \\
\hline Bautmans et al. [34] & 6 weeks/(3/week) & $2-5 \mathrm{~mm}$ & $30-40 \mathrm{~Hz}$ & $\begin{array}{l}4 \text { sets } \times 30-60 \text { s, 30-60 s rest between } \\
\text { set }\end{array}$ \\
\hline Beck et al. [35] & 32 weeks/(2/week) & $0.3 \mathrm{~g}$ & $30 \mathrm{~Hz}$ & $15 \mathrm{~min}$ (1 session), no rest \\
\hline Bogaerts et al. [37] & 47 weeks/(3/week) & $2.5-5 \mathrm{~mm}$ & $30-40 \mathrm{~Hz}$ & $\begin{array}{l}4 \text { sets sets } \times 30 s-15 \times 30 s, 15-30 s \\
\text { rest between set }\end{array}$ \\
\hline Bogaerts et al. [38] & 48 weeks mo/(3/week) & NA & NA & NA \\
\hline Boegarts [36] & 24 weeks/(3/week) & $1.6-2.2 \mathrm{~g}$ & $30-40 \mathrm{~Hz}$ & $\begin{array}{l}3 \text { sets } \times 15-60 \text { s, } 60-5 \text { s rest between } \\
\text { set }\end{array}$ \\
\hline Corrie etal. [65] & 12 weeks/(3/week) & $1.3 \mathrm{~mm}$ & $30 \mathrm{~Hz}$ & $\begin{array}{l}3 \text { to } 6 \text { sets } \times 20 \text { to } 60 \mathrm{~s}, 60 \text { s rest } \\
\text { between set }\end{array}$ \\
\hline Gomez-Cabello [42] & 44 weeks mo/(3/week) & $2 \mathrm{~mm}$ & $40 \mathrm{~Hz}$ & 10 sets $\times 45 \mathrm{~s}, 60$ s rest between set \\
\hline Kemmler etal. [44] & 88 weeks/(2/week) & NA & $25-35 \mathrm{~Hz}$ & NA \\
\hline Kennis et al. [45] & & $2.5-5 \mathrm{~mm}$ & $30-40 \mathrm{~Hz}$ & $\begin{array}{l}4 \text { sets } \times 30 \text { s till } 15 \text { sets } \times 30 s, 15-30 s \\
\text { rest between set }\end{array}$ \\
\hline Klarner et al. [46] & 48 weeks mo/(3/week) & & $35 \mathrm{~Hz}$ & 7 sets $\times 90 \mathrm{~s}, 40$ s rest between set \\
\hline Lachance [47] & 8 weeks/(2/week) & $2 \mathrm{~mm}$ & $35 \mathrm{~Hz}$ & NA \\
\hline Leung et al. [66] & 72 weeks mo/(5/week) & $2 \mathrm{~mm}$ & $35 \mathrm{~Hz}$ & 20 min, rest (NA) \\
\hline Machado et al. [48] & 10 week/(3-5/week) & $2-4 \mathrm{~mm}$ & $20-40 \mathrm{~Hz}$ & $3-8$ sets $\times 30-60$ s, rest (NA) \\
\hline Mikhael et al. [49] & 12 weeks/(3/week) & $1 \mathrm{~mm}$ & $12 \mathrm{~Hz}$ & 10 sets $\times 60 \mathrm{~s}, 60$ s rest between set \\
\hline Roelants et al. [53] & 24 weeks/(3/week) & $2.5-5 \mathrm{~mm}$ & $35-40 \mathrm{~Hz}$ & $\begin{array}{l}1-3 \text { sets } \times 30-60 \text { s of one exercise, } 60 \text { to } \\
5 \text { s rest between set }\end{array}$ \\
\hline Sitjà-Rabert et al. [64] & 6 weeks/(3/week) & $2-4 \mathrm{~mm}$ & $30-35 \mathrm{~Hz}$ & $\begin{array}{l}3 \text { sets } \times 30-60 \text { s of one exercise, } 60 \text { to } 5 \\
\text { s rest between set }\end{array}$ \\
\hline Verschueren et al. [58] & 24 weeks/(3/week) & $1.7-2.5 \mathrm{~mm}$ & $35-40 \mathrm{~Hz}$ & NA \\
\hline Verschueren et al. [57] & 18 weeks/(3/week) & $1.6-2.2 \mathrm{~g}$ & $30-40 \mathrm{~Hz}$ & $\begin{array}{l}15-60 \mathrm{~s} \times \text { Pos. exercise, } 60 \mathrm{~s} \text { till } 5 \mathrm{~min} \\
\text { restbetweenexercises }\end{array}$ \\
\hline
\end{tabular}

\section{Side-alternating sinusoidal vibration}

\begin{tabular}{|c|c|c|c|c|}
\hline Beck et al. [35] & 32 weeks/(2/week) & $2 \mathrm{~mm}$ & $12.5 \mathrm{~Hz}$ & 2 sets $\times 3 \mathrm{~min}, 60$ s rest between set \\
\hline Calderetal. [39] & 6 weeks & $2 \mathrm{~mm}$ & $20 \mathrm{~Hz}$ & 4 sets $\times 75 \mathrm{~s}, 90$ s rest between set \\
\hline Corrie et al. [65] & 12 weeks/(3/week) & $2.9 \mathrm{~mm}$ & $30 \mathrm{~Hz}$ & $\begin{array}{l}3 \text { to } 6 \text { sets } \times 20 \text { to } 60 \mathrm{~s}, 60 \mathrm{~s} \text { rest } \\
\text { between set }\end{array}$ \\
\hline Furness and Maschette [40] & 6 weeks & $0.05 \mathrm{~mm}$ & $15-25 \mathrm{~Hz}$ & 5 sets $\times 60 \mathrm{~s}, 60$ s rest between set \\
\hline Furness et al. [41] & 6 weeks/(3/week) & $1 \mathrm{~mm}$ & $15-25 \mathrm{~Hz}$ & 5 sets $\times 60 \mathrm{~s}, 60$ s rest between sets \\
\hline Iwamoto et al. [43] & 18 weeks/(2/week) & NA & $20 \mathrm{~Hz}$ & 4 min, NA rest \\
\hline Klarner et al. [40] & 48 weeks/(3/week) & $3-7 \mathrm{~mm}$ & $12,5 \mathrm{~Hz}$ & 7 sets $\times 90$ s, 40 s rest between set \\
\hline Ochi et al. [67] & 12 weeks/(3/week) & $12 \mathrm{~mm}$ & $2,5 \mathrm{~Hz}$ & $180 \mathrm{~s}$, no rest \\
\hline Raimundo etal. [50] & 32 weeks/(3/week) & $6 \mathrm{~mm}$ & $20-30 \mathrm{~Hz}$ & 3 sets $\times 60 \mathrm{~s}, 60$ s rest between set \\
\hline
\end{tabular}




\begin{tabular}{|c|c|c|c|c|}
\hline Rees et al. [51] & 8 weeks/(3/week) & $5-8 \mathrm{~mm}$ & $26 \mathrm{~Hz}$ & $\begin{array}{l}6 \text { sets } \times 45 \text { up to } 60 \text { s, } 5 \times 45 \text { up to } 80 \\
\text { s rest between set }\end{array}$ \\
\hline Rees et al. [52] & 8 weeks/(3/week) & $5-8 \mathrm{~mm}$ & $26 \mathrm{~Hz}$ & $\begin{array}{l}6 \text { sets } \times 45-80 \text { s, } 45-80 \text { s rest between } \\
\text { set }\end{array}$ \\
\hline Russo et al. [55] & 24 weeks/(2/week) & NA & $12-28 \mathrm{~Hz}$ & $\begin{array}{l}3 \text { sets } \times 60-120 \mathrm{~s}, 60 \mathrm{~s} \text { rest between } \\
\text { set }\end{array}$ \\
\hline Sievänen et al. [61] & 10 week/(2/week) & $2-8 \mathrm{~mm}$ & 12 and $18 \mathrm{~Hz}$ & $\begin{array}{l}1-5 \text { sets } \times 60-120 \mathrm{~s}, 60 \mathrm{~s} \text { rest } \\
\text { between set }\end{array}$ \\
\hline Stolzenberg et al. [56] & 36 weeks/(2/week) & NA & $22-26 \mathrm{~Hz}$ & $60-90 \mathrm{~s}$, rest (NA) \\
\hline von Stengel et al. [59] & 74 weeks/(2weeks) & $1.7-2 \mathrm{~mm}$ & $25-35 \mathrm{~Hz}$ & 6 sets $\times 60 \mathrm{~s}, 60$ s rest between set \\
\hline Zhang et al. [60] & 8 weeks/(3-5/week) & $1-3 \mathrm{~mm}$ & $25-35 \mathrm{~Hz}$ & $4-5$ sets $\times 60 \mathrm{~s}, 60$ s rest between set \\
\hline \multicolumn{5}{|c|}{ Stochastic resonance vibration } \\
\hline Kessler et al. [23] & 4 weeks/(3/week) & - & $\begin{array}{l}\text { 3-6 Hz (Noise } \\
4)\end{array}$ & 5 sets $\times 60 s, 60$ s rest between set \\
\hline Roganetal. [54] & 4 weeks/(3/week) & - & $5 \mathrm{~Hz}$ (Noise 4) & 5 sets $\times 60 s, 60$ s rest between set \\
\hline Roganetal. [20] & $\begin{array}{l}\text { Immediately } \\
\text { (acute effects) }\end{array}$ & - & $6 \mathrm{~Hz}$ (Noise 4) & 5 sets $\times 60 \mathrm{~s}, 60$ s rest between set \\
\hline
\end{tabular}

Abbreviation: mo: month, wk: week, s: seconds, Hz: hertz, NA: not available

\section{Meta-analysis}

For the meta-analysis 37 studies were included and data were available for IMVC, DS, power, RFD or FS outcome measurements. The effect sizes for these outcomes are summarized in Figs. $\underline{3}, \underline{4}, \underline{5}, \underline{6}, \underline{7}, \underline{8}, \underline{9}, \underline{10}, \underline{11}, \underline{12}$ and $\underline{13}$.

\section{Isometric maximal voluntary contraction: WBV vs. non- exercise control group}

Thirteen studies $[\underline{20}, \underline{23}, \underline{36}-\underline{38}, \underline{45}, \underline{46}, \underline{48}, \underline{53}, \underline{57}-\underline{59}, \underline{67}]$, including 1468 participants, reported data contributing to the comparison WBV vs. non-exercise control group, one study had two WBV-arms (vertical and side-alternating) and one control arm [46] (the number of participants in the control groups was cut in half to obtain correct numbers for the pooled analysis). The pooled overall SMD was 0.44 (95\% Cl 0.30 to 0.58 ) in favor of WBV with low heterogeneity, $1^{2} 25.9 \%$ ( $p$ $=0.176)$.

\section{Go-Go}

The subgroup analysis for SV-WBV-Go-Go showed a SMD of 0.48 (95 \% Cl 0.33 to 0.63 ) with a low heterogeneity $\left(I^{2} 10.8 \%(p=0.346)\right)$; and for SS-WBV-Go-Go a SMD of 0.69 (95\% Cl 0.32 to 1.06$)$ with a low heterogeneity, $I^{2} 9.8 \%(p=0.292)$.

\section{Slow-Go}

The subgroup analysis for SV-WBV-Slow-Go revealed a SMD of $0.14(95 \% \mathrm{Cl}-0.13$ to 0.41$)$ with a low heterogeneity, $I^{2} 5.6 \%(p=0.303)$. 


\section{No-Go}

The subgroup analysis for SR-WBV-No-Go showed a SMD of $0.27(95 \% \mathrm{Cl}-0.34$ to 0.88 ) in favour of SR- WBV with no heterogeneity, $I^{2} 0.0 \%(p=0.995)$ (Fig. 3).

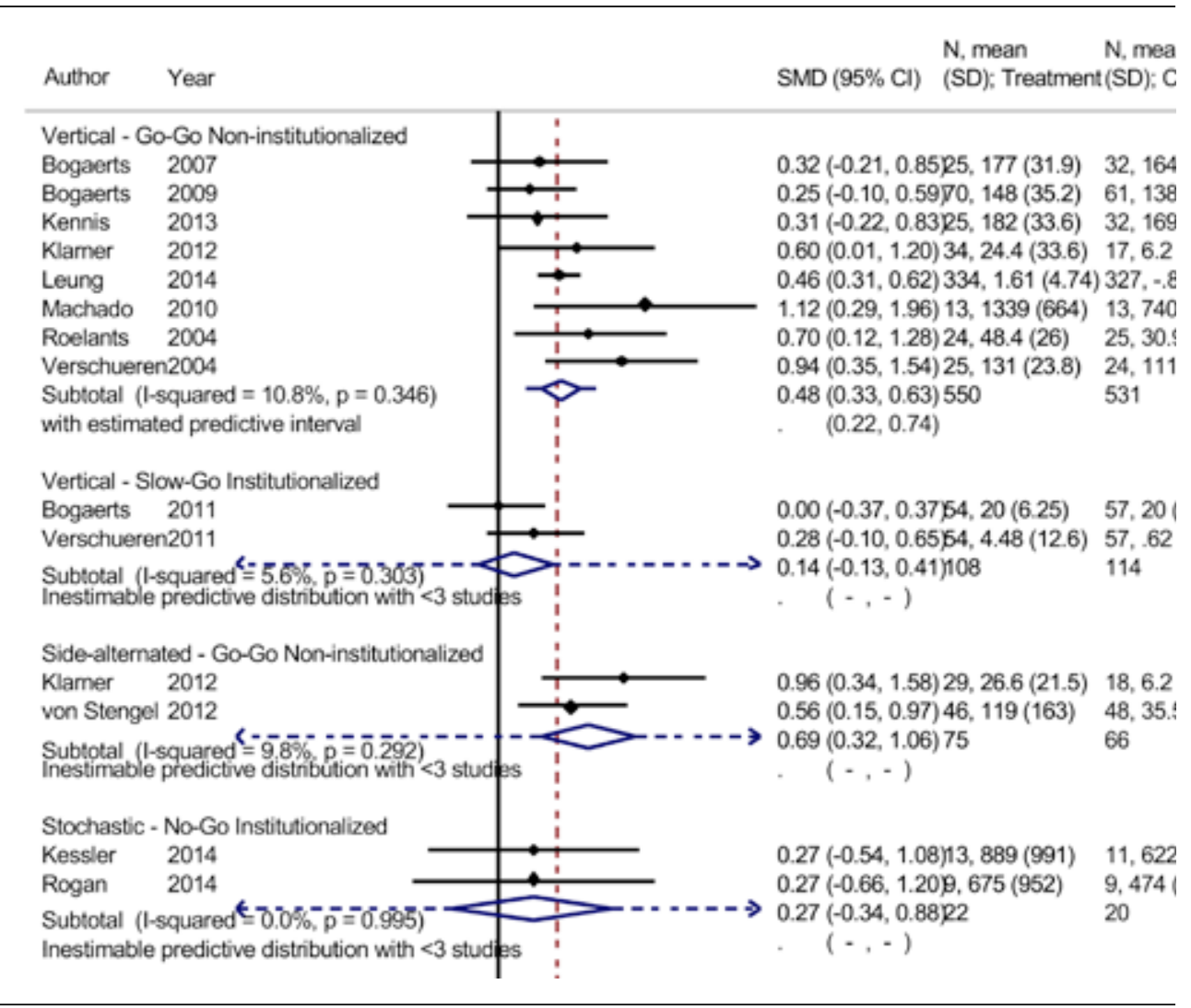

\section{Fig. 3}

Forest plot of the effects of WBV compared to no exercise on isometric strength. Values on $\mathrm{x}$-axis denote standardized mean differences.

\section{Isometric Maximal Voluntary Contraction: WBV vs. exercise group}

For the comparison WBV versus conventional exercise eight studies [37, 38, 45, 53, 58-60, 68] contributed 460 participants. The pooled overall SMD was 0.01 (95\% $\mathrm{Cl}-0.21$ to 0.22$)$ with a low heterogeneity, $I^{2}=21.9 \%(p=0.255)$.

\section{Go-Go}

The subgroup analysis for SV-WBV-Go-Go showed a SMD of -0.16 ( $95 \% \mathrm{Cl}-0.38$ to 0.06 ) with a low heterogeneity, $I^{2} 0.0 \%(p=0.966)$; SS-WBV-Go-Go showed an SMD of 0.24 ( $95 \% \mathrm{Cl}-0.17$ to $0.65)$ in favour of SS-WBV. 


\section{Slow-Go}

The subgroup analysis for SS-WBV-Slow-Go showed a SMD of $-0.04(95 \% \mathrm{Cl}-0.92$ to 0.84$)$ in favour of conventional exercise.

\section{No-Go}

The subgroup analysis for SS-WBV-No-Go showed a SMD of 0.77 (95\% Cl 0.10 to 1.44) in favour of SS-WBV.

\begin{tabular}{|c|c|c|c|c|c|c|}
\hline Author & Year & & SMO $(95 \% \mathrm{Cl})$ & $\begin{array}{l}\mathrm{N}, \text { mean } \\
\text { (SD), Treatment }\end{array}$ & $\begin{array}{l}\mathrm{N}, \text { mean } \\
\text { (SD); Control }\end{array}$ & $\%$ \\
\hline \multicolumn{7}{|c|}{ Vertical - Go-Go Non-insthusionalzed } \\
\hline Bogeerts & 2009 & & $.0 .20(-0.57,0.16)\}$ & $70,148(35.2)$ & $49,156(39.6)$ & 21.56 \\
\hline Bogaerts & 2007 & & $-0.30(-0.85,0.26)$ & $25,177(31.9)$ & 25. $186(29.4)$ & 11.79 \\
\hline Kenris & 2013 & & $-0.12(-0.68,0.43)$ & $25,182(33.6)$ & $25,186(38.6)$ & 11.88 \\
\hline Roelants & 2004 & & $-0.05(-0.64,0.54)$ & $24,48.4(26)$ & $20,49.7(21.9)$ & 10.66 \\
\hline Verschue & $\operatorname{en} 2004$ & & $-0.05(-0.62,0.52)$ & 25,131 (23.8) & 22. $133(22.1)$ & 11.28 \\
\hline Subtotal & -squared $=0.0 \%, p=0.966$ ) & & $-0.16(-0.38,0.06)$ & 169 & 141 & 67.17 \\
\hline with estin & ted predictive interval & & $(-0.52,0.20)$ & & & \\
\hline \multicolumn{7}{|c|}{ Side-altemsted - Go-Go Noninstusionalized } \\
\hline von Sten: & 2012 & & $0.24(-0.17,0.65)$ & $46,119(163)$ & $47,84.1(122)$ & 1871 \\
\hline Subtotal & Isquared $=, \ldots, p=$ ) & & $0.24(-0.17,0.65)$ & 46 & 47 & 18.71 \\
\hline with estin & ated predictive interval & & (...) & & & \\
\hline \multicolumn{7}{|c|}{ Side-altemated - Slow-Co Insthutionalized } \\
\hline Ochi & 2015 & & $-0.04(-0.92,0.84)$ & $10 .-8.53(1.85)$ & $10 .-8.46(1.59)$ & 5.43 \\
\hline Subtotal & lesquared $=, \%, p=)$, & $\longrightarrow$ & $-0.04(-0.92,0.84)$ & 10 & 10 & 5.43 \\
\hline with estin & ated predictive interval & & $(\ldots)$ & & & \\
\hline \multicolumn{7}{|c|}{ Side-allemated - No-Go Institutionalized } \\
\hline Znang & 2014 & & $0.77(0.10,1.44)$ & $19,11.2(2.03)$ & $18.9 .76(1.52)$ & 8.69 \\
\hline Subtotal & Isquared $=, \%, p=$, & $\longrightarrow$ & $0.77(0.10,1.44)$ & 19 & 18 & 869 \\
\hline with estin & ated predictive interval & & (..) & & & \\
\hline $\begin{array}{l}\text { Overall ( } \\
\text { with estin }\end{array}$ & $\begin{array}{l}\text { squared }=21.9 \%, p=0.255 \text { ) } \\
\text { ated predictive interval }\end{array}$ & & $\begin{array}{r}0.01(-0.21,0.22) \\
\quad(-0.44,0.45)\end{array}$ & 244 & 216 & 10000 \\
\hline \multicolumn{7}{|c|}{ NOTE: Weights are from random eflects analysis } \\
\hline & 111 & $T_{T}+1 / 1$ & $T$ & & & \\
\hline & $-1 \cdot .75 \cdot .5 \cdot .250$ & .25 .5 .751 & 2 & & & \\
\hline \multicolumn{3}{|c|}{ Favors Control Favors WBV } & & & & \\
\hline
\end{tabular}

\section{Fig. 4}

Forest plot of the effects of WBV compared to exercise on isometric strength. Values on $\mathrm{x}$-axis denote standardised mean differences.

\section{Dynamic Strength: WBV vs. non-exercise control}

Comparing dynamic strength in WBV versus non-exercise controls, six studies $[45,49,51,53$, $57,58]$ contributed with a total of 312 participants. The pooled overall SMD was $0.34(95 \% \mathrm{Cl}$ 0.06 to 0.61 ), which was statistically significant in favour of the WBV group, with low heterogeneity ( I $\left.^{2} 26.7 \%, p=0.234\right)$. 


\section{Go-Go}

The subgroup analysis for SV-WBV-Go-Go showed a SMD of 0.47 (95\% Cl 0.06 to 0.88 ) in favour of SV-WBV with a low heterogeneity, $I^{2} 38.6 \%, p=0.180$. The subgroup analysis for SS-WBV-GoGo showed a SMD of $0.38(95 \% \mathrm{Cl}-0.34$ to 1.11$)$ in favour of SS-WBV.

\section{Slow-Go}

The subgroup analysis for SV-WBV-Slow-Go showed a SMD of 0.09 (95\% Cl -0.28 to 0.46$)$.

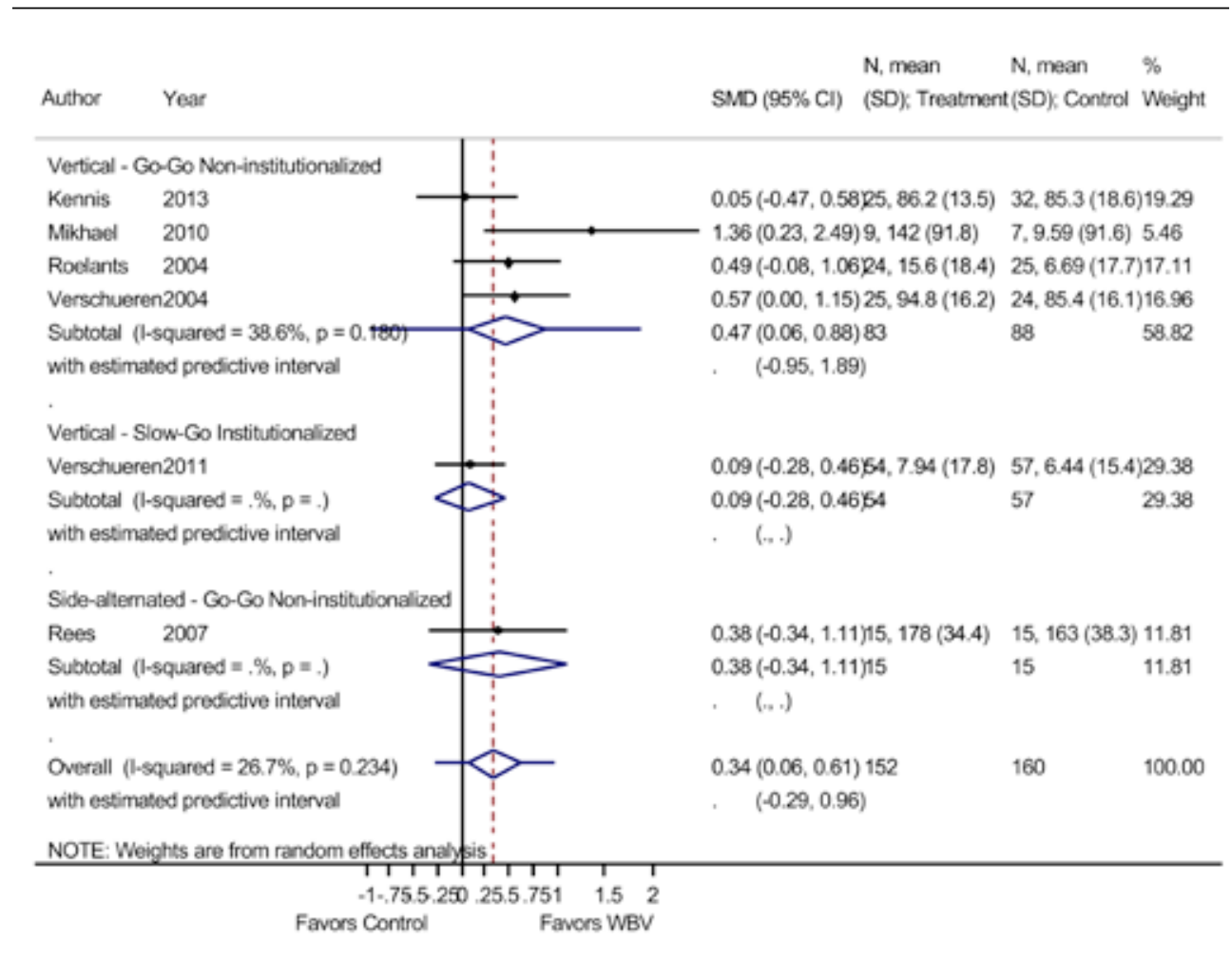

\section{Fig 5.}

Forest plot of the effects of WBV compared to no exercise on dynamic strength. Values on x-axis denote standardised mean differences.

\section{Dynamic strength: WBV vs. conventional exercise}

For the comparison WBV versus conventional exercise seven studies [45, 50-52, 58, 59, 71] contributed with a total of 245 participants. The pooled overall SMD was $0.08(95 \% \mathrm{Cl}-0.34$ to 0.17 ), statistically non-significant, thus, not in favour of a particular group, with no heterogeneity $\left(I^{2} 0.0 \%, p=0.539\right)$. 


\section{Go-Go}

The subgroup analysis for SV-WBV-Go-Go showed a SMD of -0.25 (95\% Cl -0.59 to 0.08 ) in favour of exercise, with no heterogeneity, $1^{2} 0.0 \%(p=0.639)$. The subgroup analysis for SSWBV-Go-Go showed a SMD of $0.16(95 \% \mathrm{Cl}-0.27$ to 0.60$)$ statistically not significant in favour of SS-WBV with no heterogeneity, $I^{2} 0.0 \%(p=0.415)$.

\section{Slow-Go}

The subgroup analysis for SV-WBV-Slow-Go showed a SMD of 0.08 (95\% Cl -0.77 to 0.94$)$.

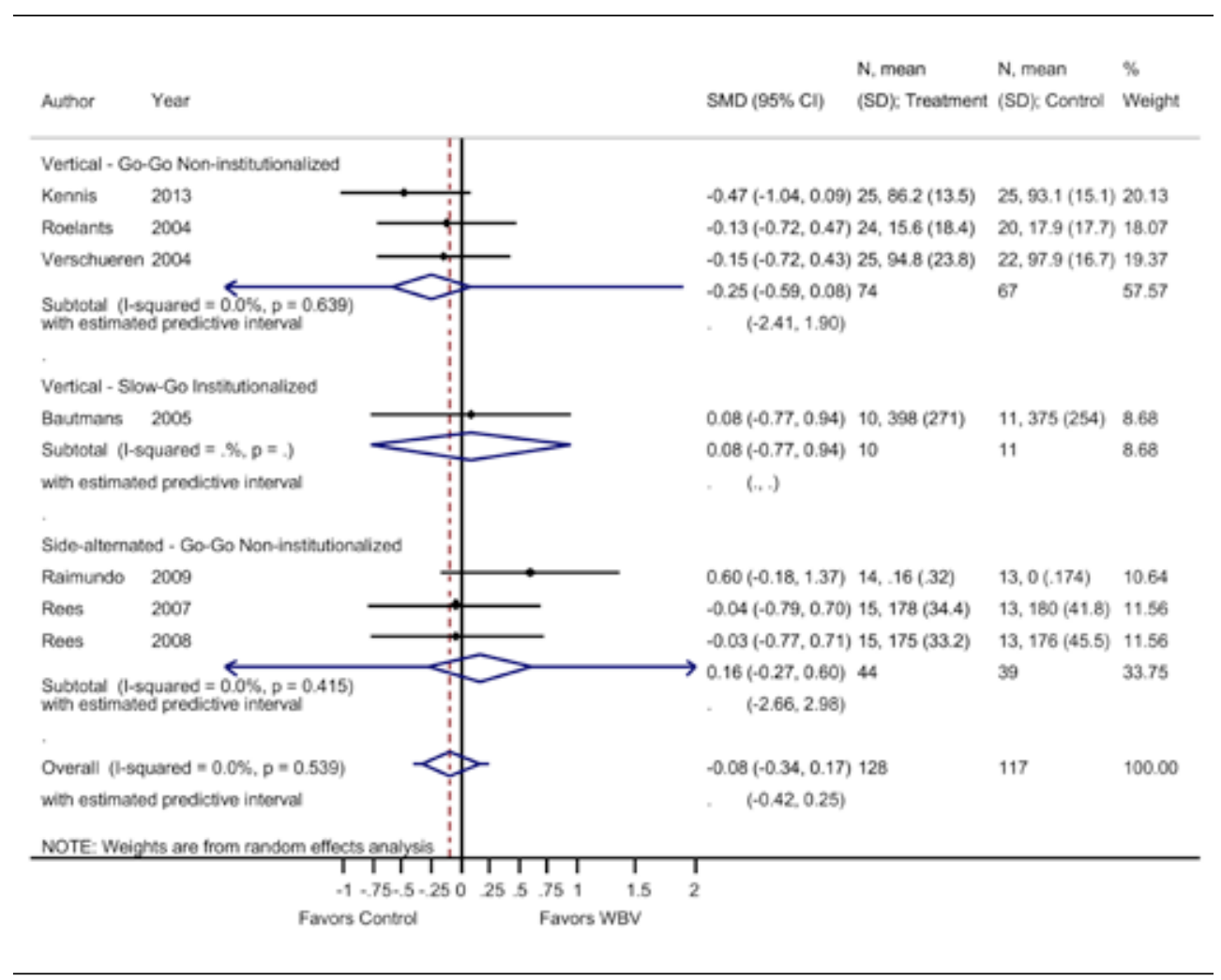

\section{Fig. 6.}

Forest plot of the effects of WBV compared to exercise on dynamic strength. Values on $\mathrm{x}$-axis denote standardised mean differences.

\section{Power: WBV vs. non-exercise control}

For the outcome power and the comparison WBV versus non-exercise control five studies [48, $49,55,56,66]$ contributed with a total of 186 participants. The control group of the Corrie et al. [66] study contributed to two subgroup analyses, therefore, we cut the number of participants in the control group in half to avoid incorrect standard errors. 


\section{Go-Go}

The pooled overall SMD was $0.22(95 \% \mathrm{Cl}-0.19$ to 0.64$)$, statistically non-significant in favour of the WBV group, with a moderate heterogeneity $\left(I^{2} 44.7 \%, p=0.107\right)$.

The subgroup analysis for SV-WBV-Go-Go showed a SMD of -0.45 (95\% Cl -1.14 to 0.25 ), with low heterogeneity, $I^{2} 17.3 \%$ ( $\left.p=0.271\right)$. SS-WBV-Go-Go showed a SMD of $0.50(95 \% \mathrm{Cl} 0.07$ to 0.92$)$ statistically significant in favour of SS-WBV with no heterogeneity, $I^{2} 0.0 \%(p=0.405)$.

\section{Slow-Go}

The subgroup analysis for SV-WBV-Slow-Go showed a SMD of 0.73 (95\% Cl -0.09 to 1.55). SS-WBVSlow-Go showed a SMD of 0.31 ( $95 \% \mathrm{Cl}-0.49$ to 1.12$)$ statistically not significant in favour of SS-WBV.

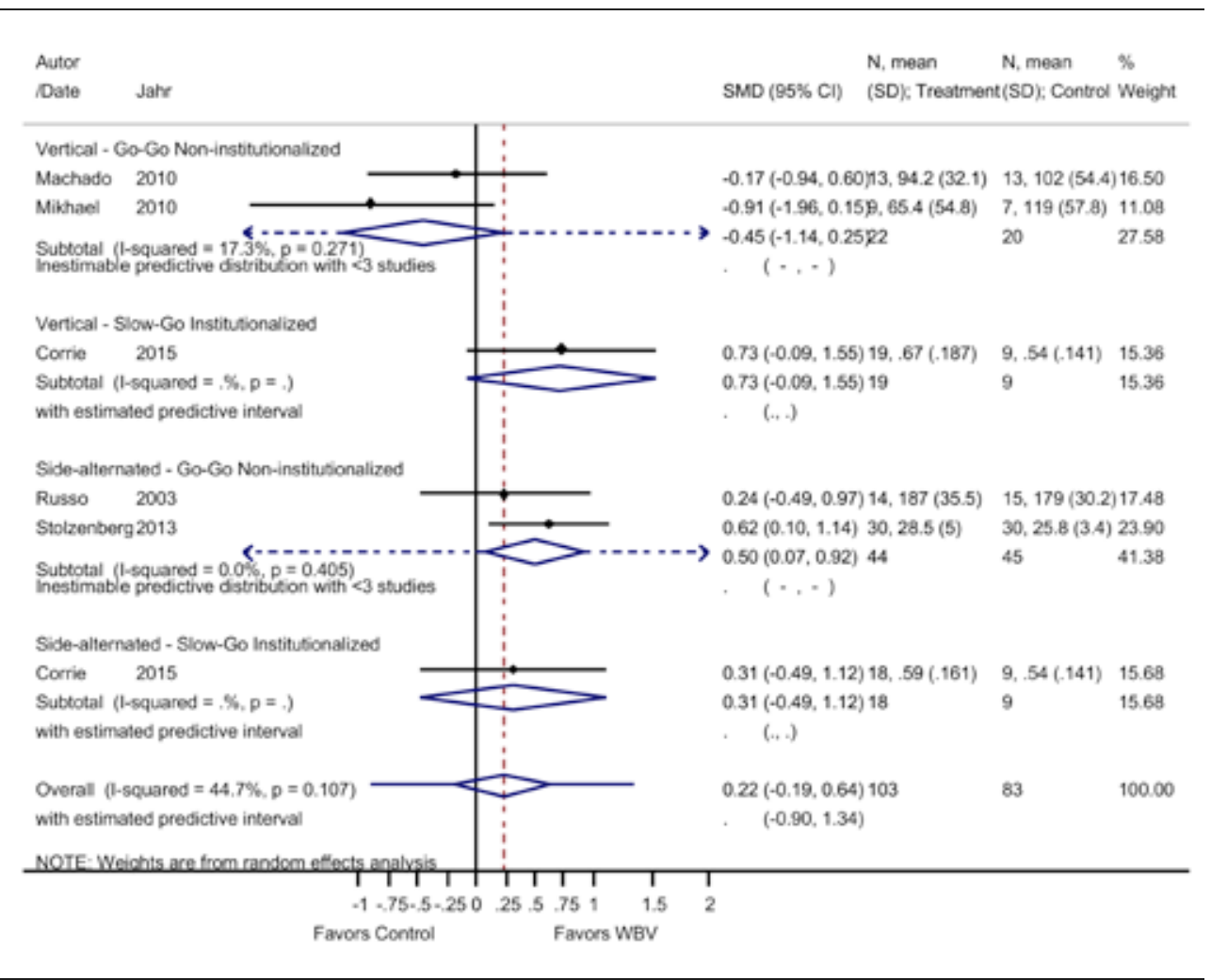

\section{Figure 7}

Forest plot of the effects of WBV compared to no exercise on power.

\section{Power: WBV vs. conventional exercise}

Three studies $[34,50,52]$ with 76 participants contributed to the comparison SS-WBV versus conventional exercise. The SMD was 0.39 (95\% Cl -0.11 to 0.90$)$, statistically not significant in favour of the WBV group. 


\section{Go-Go}

The subgroup analysis for SS-WBV-Go-Go showed a SMD of 0.63 (95\% Cl 0.08 to 1.17), statistically significant in favour of SS-WBV with no heterogeneity, $I^{2} 0.0 \%(p=0.879)$.

\section{Slow-Go}

The subgroup analysis for SV-WBV-Slow-Go showed a SMD of -0.17 (95\% Cl -1.03 to 0.69), statistically not significant in favour of conventional exercise.

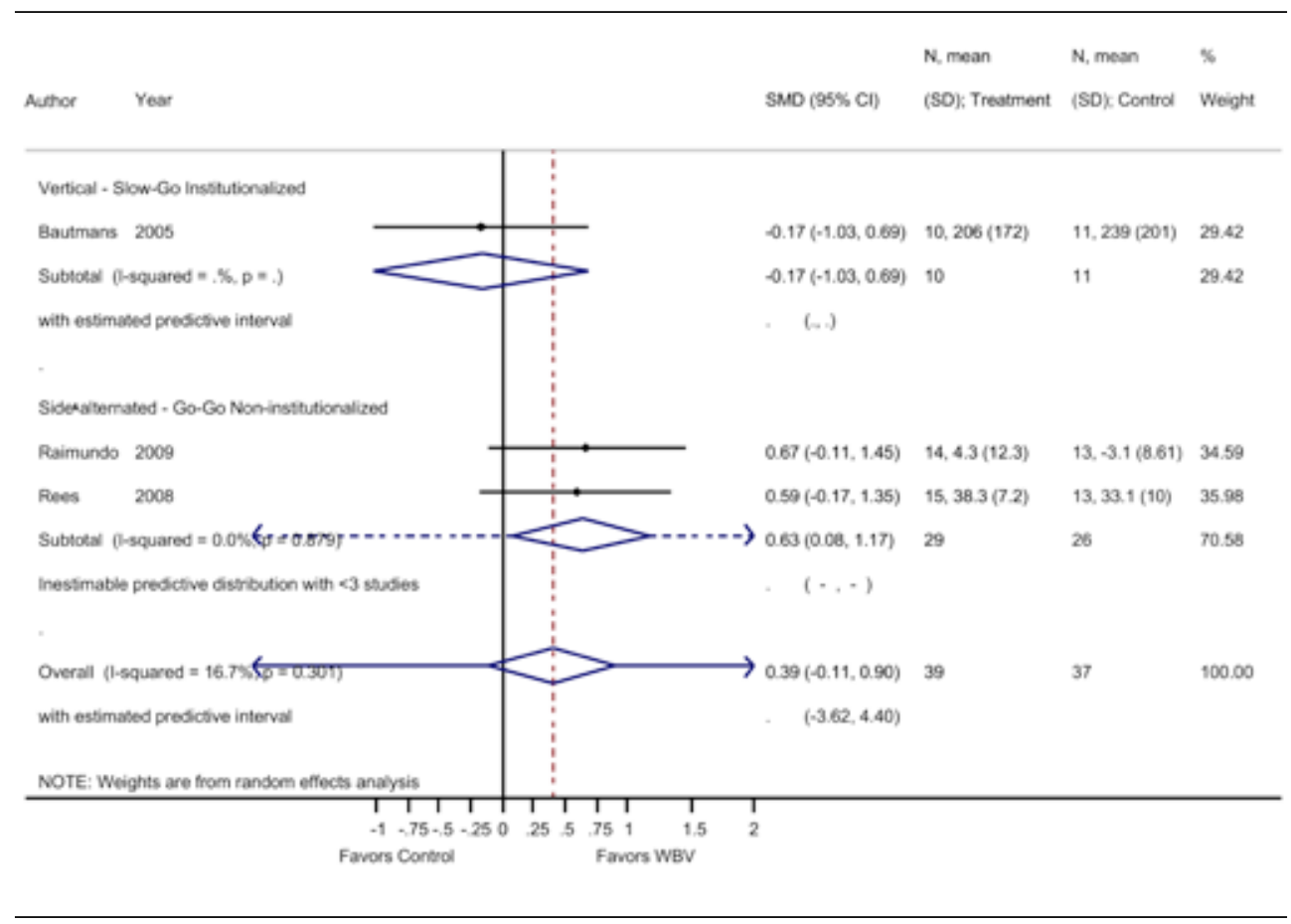

\section{Figure 8.}

Forest plot of the effects of WBV compared to exercise on power.

\section{Rate of Force Development: WBV vs. non-exercise control}

For the outcome rate of force development and the comparison WBV versus non-exercise control eight studies $[34,37,45,46,55,56,66,71]$ contributed with a total of 500 participants. The control groups of two studies $[46,66]$ contributed to two subgroup analyses, therefore, we divided the number of participants in the control groups in half. The pooled overall SMD was 0.33 (95\% Cl 0.14 to 0.52 ), statistically significant in favour of the WBV group with low heterogeneity $\left(I^{2} 6.1 \%, p=0.385\right)$. 


\section{Go-Go}

The subgroup analysis for SV-WBV-Go-Go showed a SMD of 0.39 (95\% Cl 0.08 to 0.71 ) in favour of conventional exercise with no heterogeneity, $I^{2} 0.0 \%(p=0.455)$. SS-WBV-Go-Go showed a SMD of 0.40 (95\% $\mathrm{Cl} 0.16$ to 0.64$)$ and statistically significant in favour of SS-WBV with no heterogeneity, $\mathrm{I}^{2} 0.0 \%(\mathrm{p}=0.516)$.

\section{Slow-Go}

The subgroup analysis for SV-WBV-Slow-Go showed a SMD of -0.45 (95\% Cl -1.25 to 0.36$)$. The subgroup analysis for SS-WBV-Slow-Go showed a SMD of 0.00 ( $95 \% \mathrm{Cl}-0.80$ to 0.80 ).

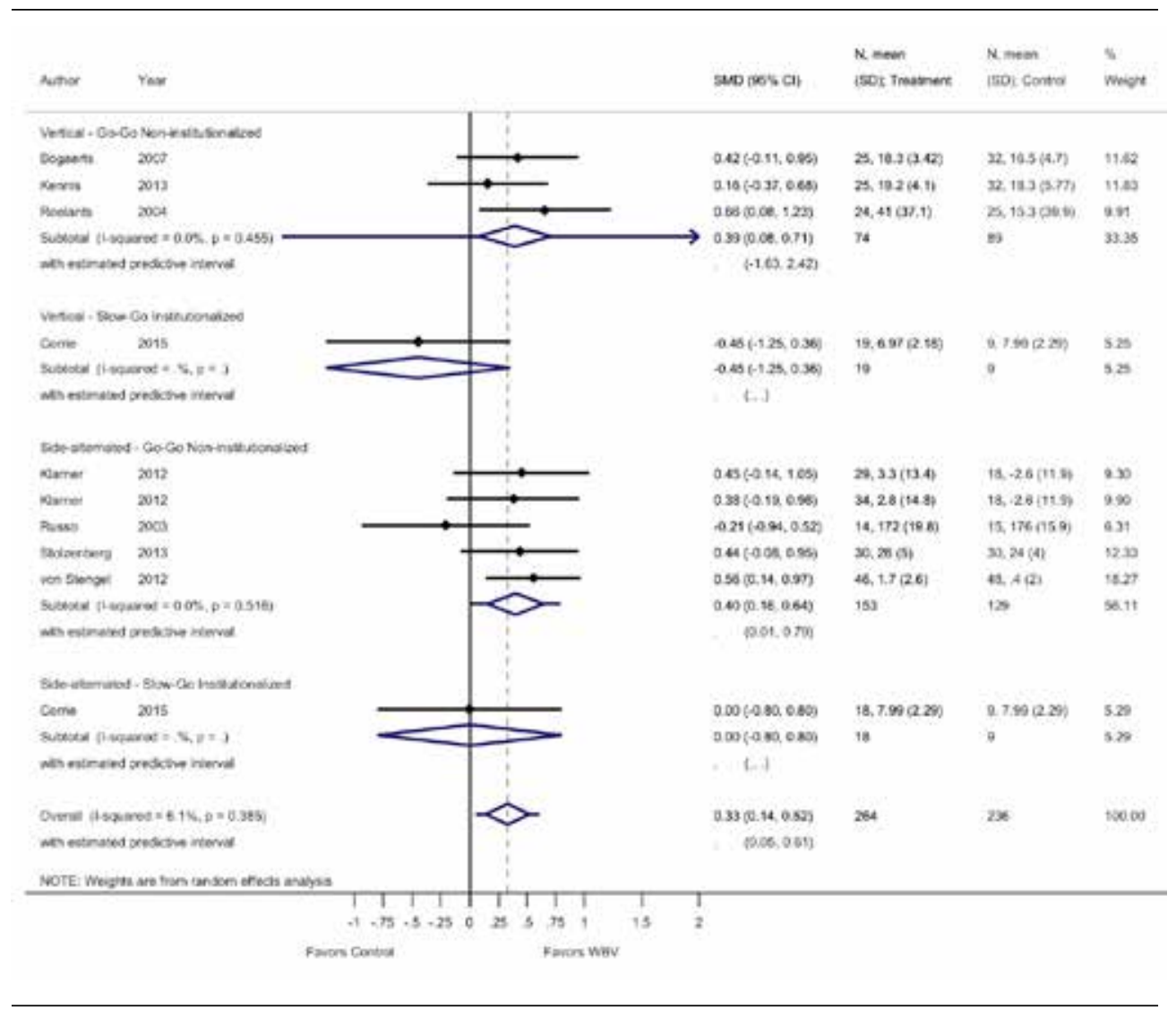

\section{Figure 9.}

Forest plot of the effects of WBV compared to no exercise on rate of force development

\section{Rate of force development: WBV vs. conventional exercise}

For the comparison WBV versus conventional exercise six studies [34, 37, 45, 50, 59, 71] contributed with a total of 285 participants. The pooled overall SMD was 0.13 ( $95 \% \mathrm{Cl}-0.11$ to 0.36 ), statistically non-significant in favour of the WBV group, with no heterogeneity $\left(I^{2} 0.0 \%, p=0.452\right)$. 


\section{Go-Go}

The subgroup analysis for SV-WBV-Go-Go showed a SMD of $-0.06(95 \% \mathrm{Cl}-0.39$ to 0.26$)$ in favour of exercise, with no heterogeneity, $I^{2} 0.0 \%(p=0.805)$. SS-WBV-Go-Go showed a SMD of 0.40 (95\% Cl 0.04 to 0.76 ), statistically significant in favour of SS-WBV with no heterogeneity, $I^{2}$ $0.0 \%(p=0.405)$.

\section{Slow-Go}

The subgroup analysis for SV-WBV-Slow-Go showed a SMD of -0.10 (95\% Cl -0.95 to 0.76$)$.

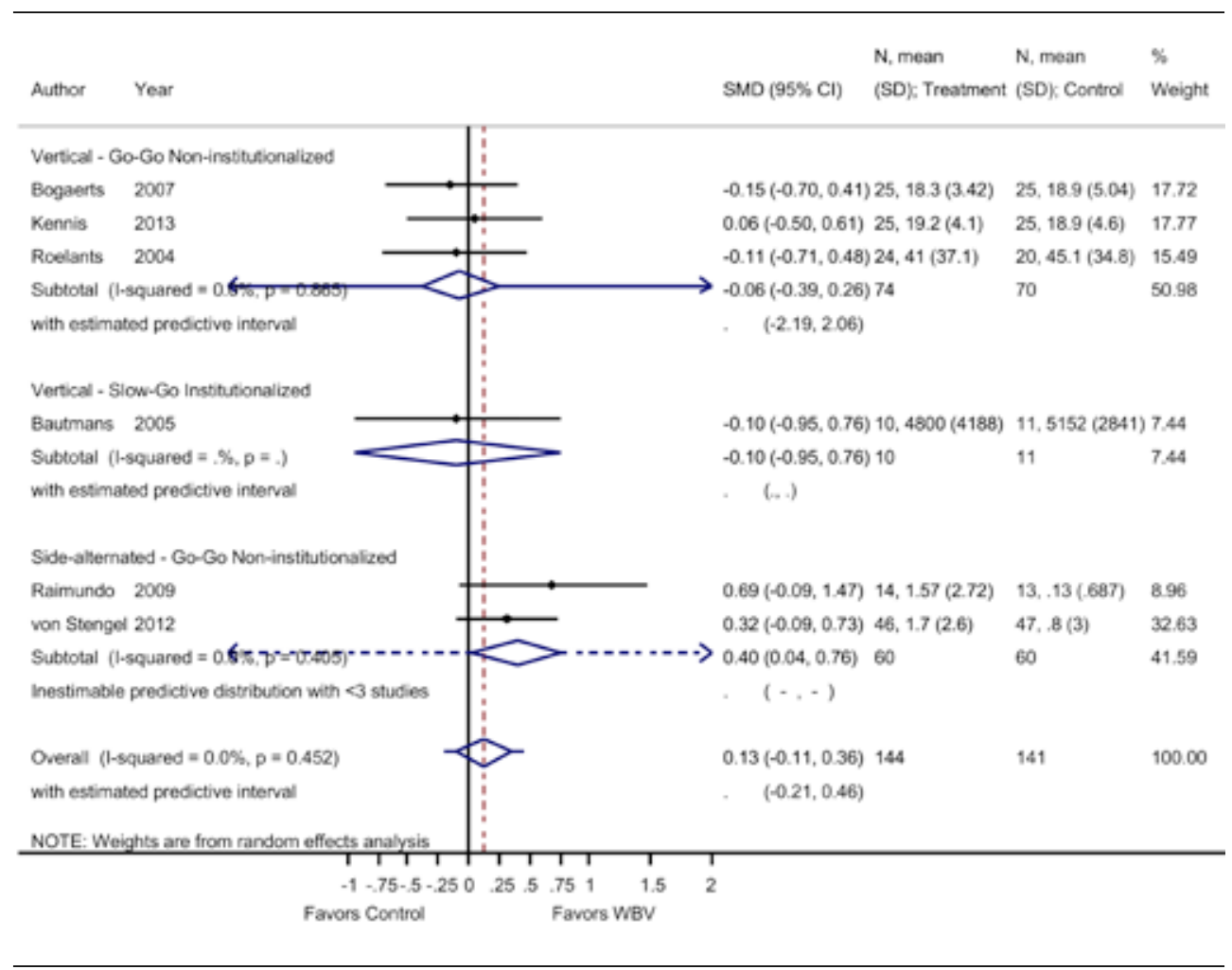

\section{Figure 10}

Forest plot of the effects of WBV compared to exercise on rate of force development.

\section{Functional Strength: WBV vs. non-exercise control}

For the outcome functional strength and the comparison WBV versus non-exercise control 17 studies $[20,23,35,37,39-43,45,49,51,54-56,59,70,71]$ contributed with a total of 565 participants (one study has SS-WBV and SV-WBV versus control, therefore we cut the number of participants of the control group in half) [35] The pooled overall SMD was 0.40 (95\% Cl 0.20 to 0.60), statistically nonsignificant in favour of the WBV group with low heterogeneity $\left(1^{2} 24.6 \%, p=0.159\right)$. 


\section{Go-Go}

The subgroup analysis for SV-WBV-Go-Go showed an SMD of 0.25 (95\% Cl -0.189 to 0.67), statistically non-significant in favour of exercise with no heterogeneity, $I^{2} 0.0 \%(p=0.893)$. SSWBV-Go-Go showed a SMD of 0.42 ( $95 \% \mathrm{Cl} 0.13$ to 0.71 ) statistically significant in favour of SS-WBV with low heterogeneity, $\left.\right|^{2} 27.5 \%(p=0.209)$.

\section{Slow-Go}

The subgroup analysis for SV-WBV-Slow-Go showed an SMD of -0.33 (95\% Cl -1.26 to 1.91) with high heterogeneity, $I^{2} 87.3 \%(p=0.005)$. The subgroup analysis for SS-WBV-Slow-Go showed a SMD of $0.41(95 \% \mathrm{Cl}-0.40$ to 1.21$)$, statistically non-significant in favour of SS-WBV with moderate heterogeneity, $\mathrm{I}^{2} 58.4 \%(p=0.121)$.

\section{No-Go}

The subgroup analysis for SS-WBV-No-Go showed a SMD of 0.24 (95\% Cl -0.85 to 1.34). The subgroup analysis for SR-WBV-Slow-Go showed a SMD of 0.97 (95\% Cl -0.07 to 2.00). The subgroup analysis for SR-WBV-No-Go showed a SMD of 0.50 (95\% Cl -0.32 to 1.33 ), statistically non-significant in favour of SR-WBV, with a moderate heterogeneity, $I^{2} 42.5 \%(p=0.187)$. 


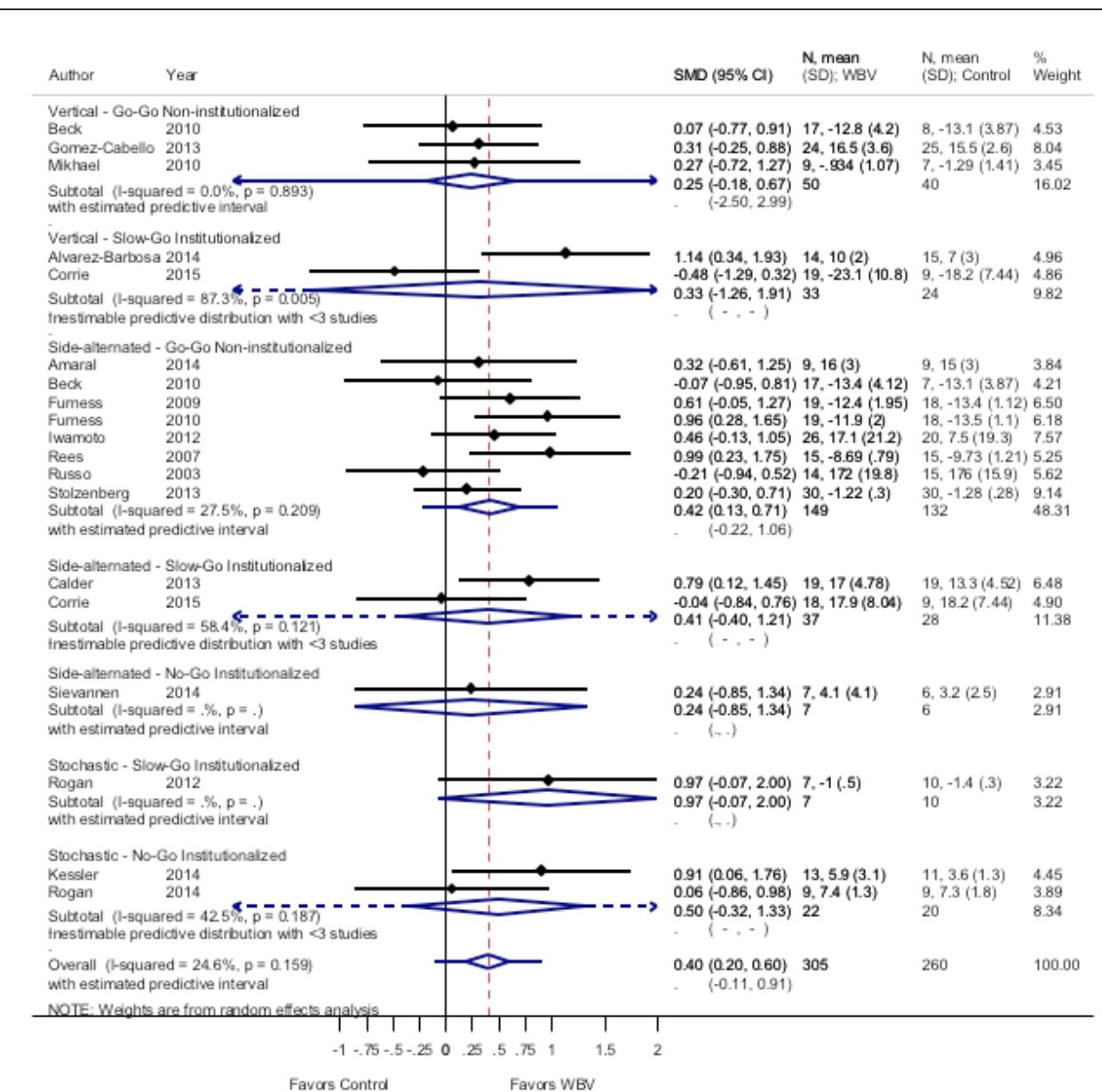

Figure 11.

Forest plot of the effects of WBV compared to no exercise on functional strength.

\section{Functional strength: WBV vs. conventional exercise}

For the comparison WBV versus conventional exercise five studies [47, 50, 51, 60, 64] contributed with a total of 306 participants. The pooled overall SMD was $-0.20(95 \% \mathrm{Cl}-0.67$ to 0.27 ), statistically non-significant in favour of the conventional exercise group with substantial heterogeneity $\left(I^{2} 70.0 \%, p=0.010\right)$.

\section{Go-Go}

The subgroup analysis for SV-WBV-Go-Go showed a SMD of -0.52 (95\% Cl -1.06 to 0.02 ), statistically non-significant in favour of conventional exercise. The subgroup analysis for SSWBV-Go-Go showed a SMD of $-0.50\left(95 \% \mathrm{Cl}-2.01\right.$ to 1.02) with high heterogeneity, $\mathrm{I}^{2} 86.3 \%$ ( $\mathrm{p}$ $=0.007)$. 


\section{Slow-Go}

The subgroup analysis for SV-WBV-Slow-Go showed a SMD of -0.07 (95\% Cl -0.38 to 0.24$)$.

\section{No-Go}

The subgroup analysis for SS-WBV-No-Go showed a SMD of $0.44(95 \% \mathrm{Cl}-0.21$ to 1.09$)$, statistically non-significant in favour of SS-WBV.

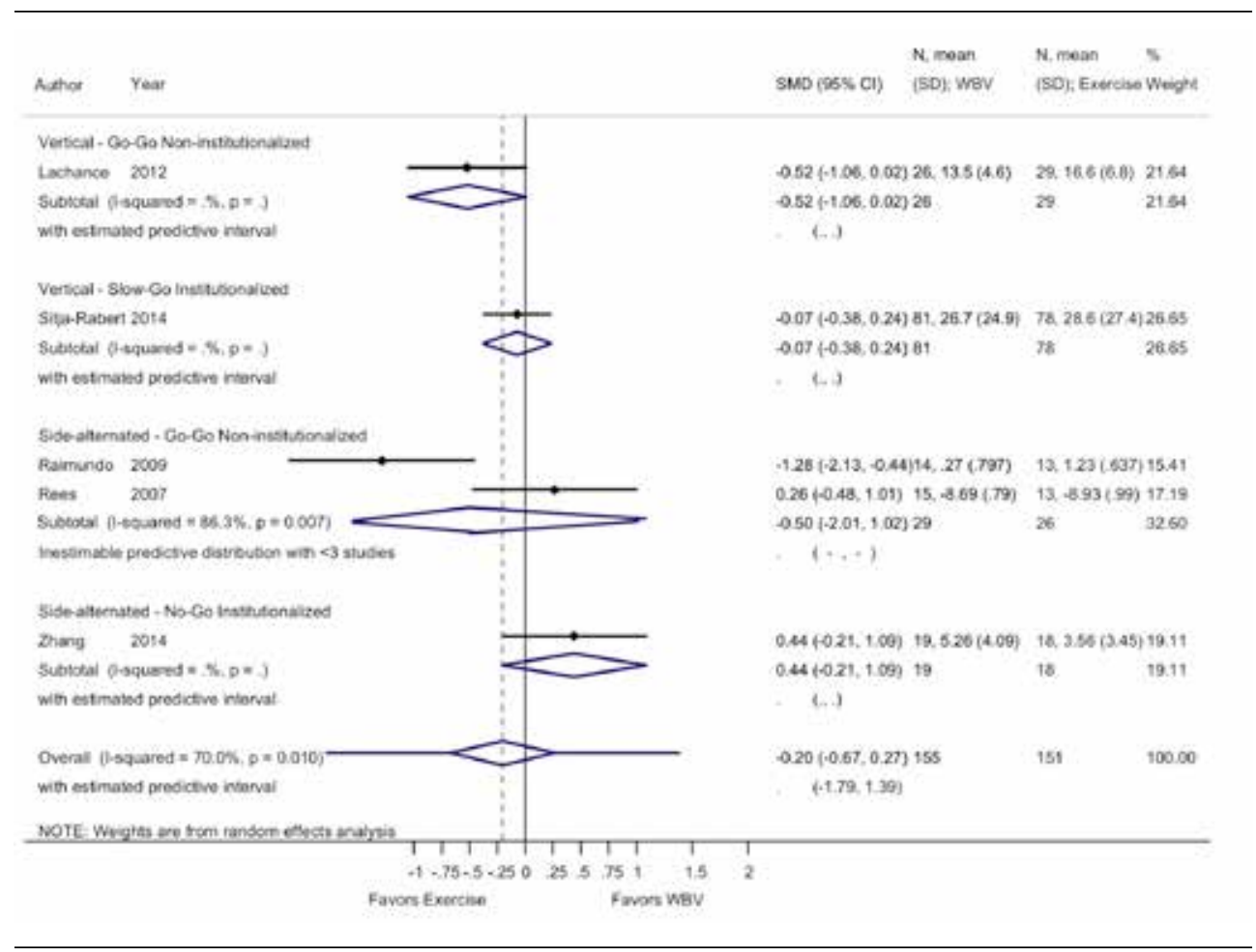

Figure 12.

Forest plot of the effects of WBV compared to exercise on functional strength.

Over all comparisons, only the comparisons of WBV versus non-exercise control for the outcomes isometric strength had a significant Egger's test $(p=0.002)$ for small study bias. Funnel plots for individual comparison are not shown. Figure 13 shows a panel of the funnel plots for all comparisons. 


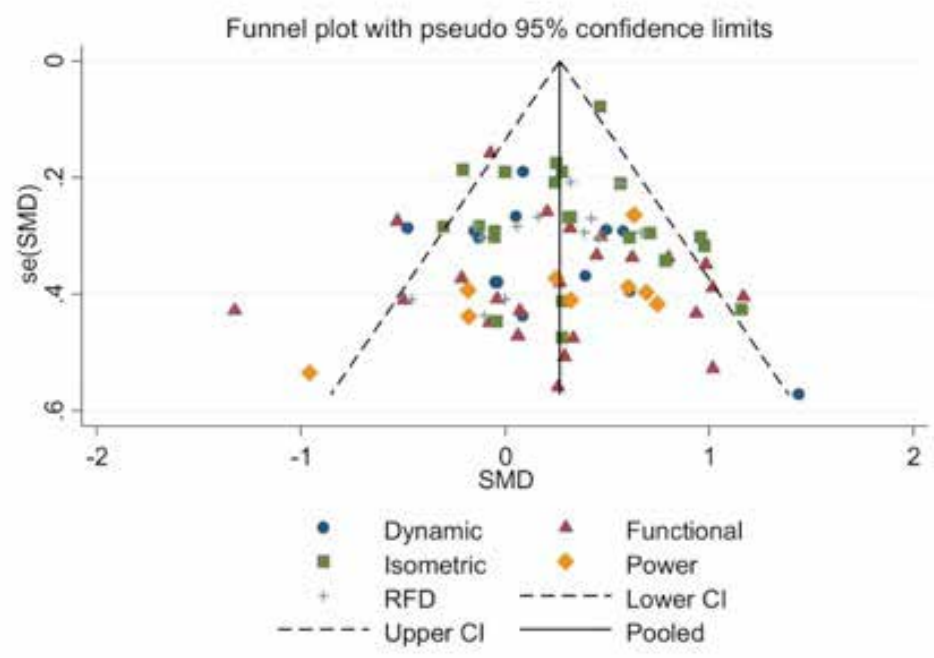

\section{Figure 13.}

Funnel plot

The effect sizes in the studies with participants in the No-Go group (SMD of 0.47, 95\% Cl 0.16 to $0.78, I^{2} 0.00 \%, p<0.792$ ) were higher compared to the Go-Go (SMD 0.26, 95\% Cl 0.15 to 0.63, $\mathrm{I}^{2} 48.9 \%, \mathrm{p}<0.001$ ) and the Slow-Go groups (SMD of $0.14,95 \% \mathrm{Cl}-0.04$ to $0.33, \mathrm{I}^{2} 26.6 \%, \mathrm{p}=$ 0.121) (see Table 3 and Appendix 2, 3 and 4). 
Table 3.

Overview of the utilization of physical performance status

\begin{tabular}{|c|c|c|c|c|c|}
\hline & \multicolumn{2}{|l|}{ Go-Go } & \multicolumn{2}{|l|}{ Slow-Go } & \multirow[t]{2}{*}{ No-Go } \\
\hline & VS-WBV & & VS-WBV & & \\
\hline 1 & Amaral et al. [65] (SA) FS & 1 & Alvarez et al. [62] FS & & \\
\hline 2 & Beck et al. [35] FS & 2 & Bautmans [34] DS, Power, RFD & & \\
\hline 3 & Boegarts et al. [37] IMVC, RFD & 3 & Boegarts et al. [36] IMVC & & \\
\hline 4 & Boegarts et al. [38] IMVC & 4 & Corrie et al. [6] Power, FS, RFD & & \\
\hline 5 & Gomez-Cabello et al. [42] FS & 5 & Sitja-Rabert et al. [70] FS & & \\
\hline 6 & $\begin{array}{l}\text { Kemmler et al. [44] IMVC, Power, } \\
\text { RFD }\end{array}$ & 6 & Verscheuern et al. [57] IMVC, DS & & \\
\hline 7 & Kennis et al. [45] IMVC, DS, RFD & & & & \\
\hline 8 & Klarner et al. [46] IMVC, RFD & & & & \\
\hline 9 & Lachane [47] FS & & & & \\
\hline 10 & Leung et al. [67] IMVC & & & & \\
\hline 11 & Machado et al. [48] MVC, Power & & & & \\
\hline 12 & Mikhael et al. [49] DS, Power, FS & & & & \\
\hline 13 & Roelants et al. [53] IMVC, DS, RFD & & & & \\
\hline \multirow[t]{2}{*}{14} & Verscheuren et al. [58] IMVC, DS & & & & \\
\hline & SS-WBV & & SS-WBV & & SS-WBV \\
\hline 1 & Beck et al. [35] FS & 1 & Calder et al. [39] FS & 1 & Sievänen et al. [61] FS \\
\hline 2 & Furness and Maschette [40] FS & 2 & Corrie et al. [66] Power, RFD, FS & 2 & Zhang et al. [60] IMVC, \\
\hline 3 & Furness et al. [41] FS & 3 & Ochi et al. [68] IMVC & & FS \\
\hline 4 & Iwamoto et al. [43] FS & & & & \\
\hline 5 & Klarner et al. [46] IMVC, RFD & & & & \\
\hline 6 & $\begin{array}{l}\text { Raimundo et al. [50] DS, } \\
\text { Power, RFD; FS }\end{array}$ & & & & \\
\hline 7 & Rees et al. [51] DS, FS & & & & \\
\hline 8 & Rees et al. [52] DS, Power & & & & \\
\hline 9 & Russo et al. [55] DS, RFD & & & & \\
\hline 10 & $\begin{array}{l}\text { Stolzenberg et al. [56] Power, } \\
\text { RFD. FS }\end{array}$ & & & & \\
\hline \multirow[t]{6}{*}{11} & von Stengel et al. [59] IMVC, RFD & & & & \\
\hline & & & SR-WBV & & SR-WBV \\
\hline & & 1 & Rogan et al. [54] FS & 1 & $\begin{array}{l}\text { Kessler et al. [23] IMVC, } \\
\text { IRFD, FS }\end{array}$ \\
\hline & & & & 2 & $\begin{array}{l}\text { Rogan et al. [20] IMVC, } \\
\text { IRFD, FS }\end{array}$ \\
\hline & $\begin{array}{l}\text { SMD of } 0.26 \text { (95\% Cl } 0.15 \text { to } 0.63) \\
\left.\right|^{2} 48.9 \%, p<0.001\end{array}$ & & $\begin{array}{l}\text { SMD of } 0.14(95 \% \mathrm{Cl}-0.04 \text { to } \\
0.33)\end{array}$ & & SMD of $0.47(95 \% \mathrm{Cl} 0.16$ \\
\hline & & & $\mathrm{p}^{2} 26.6 \%, p=0.121$ & & $1^{2} 0.00 \%, p<0.792$ \\
\hline
\end{tabular}

\section{Discussion}

We hypothesized that WBV differently effects on measures of strength and power in Go-Go, Slow-Go and No-Go. This systematic review on muscle-strength-related outcomes of WBV in healthy elderly participants included 37 studies in a final analysis, most of which were studies with small sample sizes. The main findings were that WBV showed low to moderate effects in GoGo, Slow-Go and No-Go when compared to non-exercising control groups on proxies of muscle strength in older adults. Furthermore, compared to groups performing more conventional types of exercise, WBV had only small and mostly non-significant advantages. Although only a few studies evaluated the effects of WBV in samples of elderly participants in need of care (classified 
as No-Go [10] in this review), the highest effect sizes favouring WBV were found in these studies. The few studies that evaluated SR-WBV also resulted in high effect sizes in favour of this type of WBV in No-Go. These findings seem to confirm our hypothesis. However, when we summarized the effect sizes of the meta-analysis and intended to perform statistical analysis on these data that would either refute or confirm our hypothesis, the amount of data was not big enough and the groups too unbalanced in size to allow a credible analysis to be performed. Further studies in No-Go are, therefore, warranted and needed with the various types of WBV. This means regarding our aim to give recommendations on available evidence for practical use the inference must be that at present no recommendations can be given for the most effective vibration mode in elderly persons.

Our review classified the physical capacities of the included participants (i.e. in "Go-Go", "SlowGo", and "No-Go" [10]) and analysed studies with WBV versus non-exercising control separately from studies comparing WBV versus other types of conventional strength training exercise, as recommended by Orr [68]. Further- more, we separately analyzed studies using vertical, side- alternating and stochastic resonance WBV. The reason for these distinctions relate to the assumption that initial fitness when entering a training program together with the training content may differently effect on training outcomes. Physical fitness includes health-related (cardiorespiratory endurance, muscular endurance, muscular strength, flexibility and body composition) and skill- related components (agility, coordination, balance, speed, reaction time and power) [69]. Although exercise recommendations have been published for older adults; e.g. the American College of Sports Medicine (ACSM) [70] guidelines recommend that older adults should undertake 30 min of moderate intensity, aerobic exercise or activity, five times per week to incur any health benefits, the complex interactions present in various sub-populations of older adults preclude the definition of specific, detailed exercise prescriptions. Furthermore, the number of older people fulfilling the ACSM requirement is rather small and most likely even lower amongst those with low levels of functioning [71]. It seems fair to assume that when principles of exercise training are applied to the development of exercise protocols, clinicians in practical settings can have greater confidence that non-significant research findings reflect deficiencies in exercise efficacy rather than deficiencies in exercise prescription [72]. It is thereby important, however, to consider low baseline fitness and mobility levels in pre-frail or frail or rather untrained elderly when starting an exercise program. Based on the findings of this systematic review it seems that the use of (SR)-WBV is valuable for untrained or frail elderly where the neuromuscular systems might not be able withstanding higher loading and long training sessions, however, with increasing levels of functioning there is a diminishing effect of the WBV interventions. Considering this it becomes clear that this systematic review only reveals first estimates for the possible effect of WBV in (pre-)frail elderly. An important next step would be the design and implementation of a sufficiently powered WBV exercise study specifically targeting (pre-)frail institutionalized elderly with a training duration of at least 2 months since this is the duration where effects of WBV training in the elderly may be expected [국. 


\section{Role of physical capacity level}

Although we did not find a systematic review on WBV that used a classification of participants in different subgroups of functioning, our conclusion is in line with similar previously expressed conclusions. Lau et al. [24] mainly focused on bone mineral density but also included strength measures. They concluded, that WBV is beneficial in elderly persons to increase muscle strength. However, because they did not use a separate analysis for different groups with different levels of physical capacities it is difficult to determine whether the effects observed are dependent on the baseline fitness of the study participants. Sitja-Rabert et al. [25] concluded that WBV was beneficial in elderly participants to improve strength. Osawa et al. [74] included both young and elderly participants and concluded that WBV in addition to exercises or to a normal lifestyle improved knee extensor strength and countermovement jump performance when compared to identical training conditions without WBV.

\section{WBV as skilling-up exercise}

Our findings seem to justify the assumption, that WBV might be applicable as a "skilling-up" exercise for elderly with low physical capacity (i.e. the No-Go group), who are considering to begin with an exercise program, but who are not yet able to perform traditional strength exercises. A short bout of vibration would produce sufficient stimuli to effect on muscle strength, power and functional tasks. There are some theoretical explanations that WBV might improve the neuromuscular drive, which improves muscular function [18, 75]. In contrary to the No-Go group, elderly persons in the Go-Go and the Slow-Go group can perform standard exercises and WBV might be an additional option among all exercise modalities. The measured effects sizes for the Go-Go and Slow-Go groups were not very high and not all outcomes showed significant differences in the included studies. For this reason, WBV should be rather used for "skilling-up" in pre-frail or frail elderly individuals.

\section{Limitations of this study}

There are some limitations of this review. The included studies presented moderate to high risk of bias, allocation concealment was not described in most studies, groups were often not similar at baseline (which is often the case in small studies), participants were blinded in only seven studies and outcome assessors were blinded in only 11 studies. With the exception of Leung et al. [66], Boegarts et al. [38], Kemmler et al. [44], von Stengel [59] $(n=151)$ and Sitjà-Rabert et al. [64], the remaining studies were small. This further increases the risk of bias. Therefore, the quality of the included studies overall was rather low.

The ability to replicate or reproduce experimental results, or reproducibility, is one of the major tenets of the scientific method. SR-WBV results considered in this review come from one research group only. It is, therefore, necessary that the published findings of this group are validated through replication by others. Until replication is done the results for SR- WBV should be interpreted with caution and, hence, this is a limitation of this review. Another limitation of our approach is the possible language bias, we only included studies in English or German and it cannot be excluded that relevant research in different languages exists. 
This review included mainly studies with "Go-Go" and "Slow-Go" participants and only few studies with participants from the "No-Go" group. As the effect might be largest in this No-Go group with SMD of 0.47 (95\% Cl 0.16 to 0.78 ), further studies should evaluate WBV in this group of elderly. Only a few studies have evaluated SR-WBV and no study has compared SR-WBV to other vibration modalities in elderly participants.

\section{Conclusions}

WBV shows beneficial effects on proxies of muscle strength in older adults, mainly in elderly with lower initial levels of functioning, suggesting that WBV can be used as a skilling-up exercise in participants. However, the review suggests that WBV has no overall treatment effect on muscle strength properties in older women and men across the whole spectrum of physical functioning. Only few studies evaluated WBV in (pre-)frail elderly. No randomized trial has examined the effects of WBV on muscle in older (pre-)frail elderly. Based on this re- view no recommendations can be given for the most effective vibration mode. Further studies with the various types of WBV in various sub-populations of elderly persons are warranted and needed to determine the most effective vibration modes.

\section{Abbrevations}

CENTRAL: Cochrane Central Register of Controlled Trials, control: control group, DS: dynamic strength; Ex: exercise group, FS: functional strength, PEDro: Physiotherapy Evidence Database; Go-Go: independent person; $1^{2}: 1^{2}$-statistic for heterogeneity, IMVC: isometric maximum voluntary contraction; No-Go: person depending permanently on assistance with severe functional limitation; RFD: rate of force development; Sham: sham group, SR-WBV: stochastic resonance whole-body vibration; SS-WBV: side-alternating sinusoidal whole-body vibration; Slow-Go: depending on support in everyday activities such as dressing, body care, eating, using the toilet, mobility, and planning the day; SMD: standardised mean differences; SV-WBV: sinusoidal vertical whole-body vibration; WBV: whole-body vibration; WHO: World Health Organization

\section{Competing interests}

The authors declare no potential conflict of interest with respect to the authorship and/or publication of this article.

\section{Authors' contributions}

SR conceived the idea of this study. SR, $\mathrm{LR}$, and $\mathrm{RH}$ participated in the conception and design of the study; SR, CJ, CW, NJS, YB, RH collected the data. RH analysed the data. SR, EDB and RH wrote the manuscript. EDB, RDB: Methodology, Supervision, critically revising the manuscript 
for important intellectual content. LR, CJ, CW, NJS, YB, EDB and $\mathrm{RH}$ were involved in drafting or revising the manuscript. All authors read and approved the final manuscript.

\section{Authors information}

${ }^{1}$ Bern University of Applied Sciences, Discipline Physiotherapy, Bern, Switzerland 2Department of Epidemiology, CAPHRI School for Public Health and Primary Care, Maastricht University, PO Box 616, 6200 MD Maastricht, The Netherlands

${ }^{3}$ Centre for Evidence Based Physiotherapy, Maastricht University, PO Box 616, 6200 MD Maastricht, The Netherlands

${ }^{4}$ Department of Health Sciences and Technology, Institute of Human Movement Sciences and Sport, ETH, Zurich, Switzerland

${ }^{5}$ School of Health Sciences, University of Applied Sciences and Arts Western Switzerland Valais (HES 


\section{References}

1. Aagaard P, Suetta C, Caserotti P, Magnusson SP, Kjaer M: Role of the nervous system in sarcopenia and muscle atrophy with aging: strength training as a countermeasure. Scand J Med Sci Sports 2010, 20(1):49-64.

2. Granacher U, Muehlbauer T, Gruber M: A qualitative review of balance and strength performance in healthy older adults: impact for testing and training. J Aging Res 2012, 2012:708905.

3. Manini TM, Clark BC: Dynapenia and aging: an update. J Gerontol A Biol Sci Med Sci 2012, 67(1):28-40.

4. Clark BC, Manini TM: Sarcopenia =/= dynapenia. J Gerontol A Biol Sci Med Sci 2008, 63(8):829-834

5. Clark BC, Manini TM: What is dynapenia? Nutrition 2012, 28(5):495-503.

6. Clark BC, Manini TM: Functional consequences of sarcopenia and dynapenia in the elderly. Curr Opin Clin Nutr Metab Care 2010, 13(3):271-276.

7. Hughes VA, Frontera WR, Wood M, Evans WJ, Dallal GE, Roubenoff R, Fiatarone Singh MA: Longitudinal muscle strength changes in older adults: influence of muscle mass, physical activity, and health. J Gerontol A Biol Sci Med Sci 2001, 56(5):B209-217.

8. Janssen I: Influence of sarcopenia on the development of physical disability: the Cardiovascular Health Study. J Am Geriatr Soc 2006, 54(1):56-62.

9. de Kort YAW: Housing for the independendtly living elderly; Theoretical backgrounds. Eindhoven, The Netherlands: Eindhoven University of Technology; 1995.

10. Zeyfang A, Braun A: [Guidelines "Diabetes mellitus in the elderly"]. MMW Fortschr Med
2009, $151(20): 33-35,37$.

11. Swiss Academy Of $M$ : Treatment and care of elderly persons who are in need of care. Swiss medical weekly 2004, 134(4142):618-626.

12. Walter-Ginzburg A, Guralnik JM, Blumstein T, Gindin J, Modan B: Assistance with personal care activities among the old-old in Israel: a national epidemiological study. J Am Geriatr Soc 2001, 49(9):1176-1184.

13. Landi F, Marzetti E, Martone AM, Bernabei $\mathrm{R}$, Onder $\mathrm{G}$ : Exercise as a remedy for sarcopenia. Curr Opin Clin Nutr Metab Care 2014, 17(1):25-31.

14. Herren K, Holz Hängärtner C, Oberli A, Radlinger L: [Cardiovascular and metabolic strain during stochastic resonance therapy in stroke patients]. Physioscience 2009 5(1):13-17.

15. Rittweger J, Beller G, Felsenberg D: Acute physiological effects of exhaustive wholebody vibration exercise in man. Clin Physio 2000, 20(2):134-142.

16. Rittweger J, Ehrig J, Just K, Mutschelknauss M, Kirsch KA, Felsenberg D: Oxygen uptake in whole-body vibration exercise: influence of vibration frequency, amplitude, and external load. Int J Sports Med 2002 , 23(6):428-432

17. Chanou K, Gerodimos V, Karatrantou K, Jamurtas $A$ : Whole-body vibration and rehabilitation of chronic diseases: a review of the literature. Journal of sports science \& medicine 2012, 11(2):187-200.

18. Rogan S, Hilfiker R: [Training methods - increase muscle strength due to wholebody vibration - force with $\mathrm{Hz}$. Sportverletz Sportschaden 2012, 26(4):185-187. 
19. Blasimann A, Fleuti U, Rufener M, Elfering A, Radlinger L: Electromyographic activity of back muscles during stochastic whole body vibration. J Musculoskelet Neuronal Interact 2014, 14(3):311-317.

20. Rogan S, Schmidtbleicher D, Radlinger L: Immediate effects after stochastic resonance whole-body vibration on physical performance on frail elderly for skilling-up training: a blind cross-over randomised pilot study. Aging Clin Exp Res 2014.

21. Nordlund MM, Thorstensson A: Strength training effects of whole-body vibration? Scand J Med Sci Sports 2007, 17(1):12-17.

22. Rittweger J: Vibration as an exercise modality: how it may work, and what its potential might be. Eur J Appl Physiol 2010, 108(5):877-904.

23. Kessler J, Radlinger L, Baur $H$, Rogan S: Effect of stochastic resonance whole body vibration on functional performance in the frail elderly: A pilot study. Arch Gerontol Geriatr 2014, 59(2):305-311.

24. Lau RW, Liao LR, Yu F, Teo T, Chung RC, Pang MY: The effects of whole body vibration therapy on bone mineral density and leg muscle strength in older adults: a systematic review and meta-analysis. Clin Rehabil 2011, 25(11):975-988.

25. Sitja-Rabert M, Rigau D, Fort Vanmeerghaeghe A, Romero-Rodriguez D, Bonastre Subirana M, Bonfill X: Efficacy of whole body vibration exercise in older people: a systematic review. Disabil Rehabil 2012, 34(11):883-893.

26. Ammann BC, Knols RH, Baschung P, de Bie RA, de Bruin ED: Application of principles of exercise training in sub-acute and chronic stroke survivors: a systematic review. BMC neurology 2014, 14:167.
27. Liberati A, Altman DG, Tetzlaff J, Mulrow C, Gotzsche PC, loannidis JP, Clarke M, Devereaux PJ, Kleijnen J, Moher D: The PRISMA statement for reporting systematic reviews and meta-analyses of studies that evaluate health care interventions: explanation and elaboration. PLoS medicine 2009, 6(7):e1000100.

28. Sayers A: Tips and tricks in performing a systematic review--chapter 4 . The British journal of general practice : the journal of the Royal College of General Practitioners 2008, 58(547):136.

29. American College of Sports Medicine Position Stand. The recommended quantity and quality of exercise for developing and maintaining cardiorespiratory and muscular fitness, and flexibility in healthy adults. Medicine and science in sports and exercise 1998, 30(6):975-991.

30. Higgins JP, Altman DG, Gotzsche PC, Juni P, Moher D, Oxman AD, Savovic J, Schulz KF, Weeks L, Sterne JA: The Cochrane Collaboration's tool for assessing risk of bias in randomised trials. BMJ 2011, 343:d5928.

31. Kakebeeke $\mathrm{TH}$, Knols $\mathrm{RH}$, de Bruin ED: Should Rehabilitation Specialists Use External Focus Instructions When Motor Learning Is Fostered? A Systematic Review. Sports 2013, 1(2):37-54.

32. Cohen J: Statistical power analysis for the behavioral sciences, 2nd edn. NJ, USA: Erlbaum, Hillsdale; 1988.

33. Higgins J, Thompson S, Deeks J, Altman D: Measuring inconsistency in meta-analysis. BMJ 2003, 327:557-560.

34. Bautmans I, Van Hees E, Lemper JC, Mets T: The feasibility of Whole Body Vibration in institutionalised elderly persons and its 
influence on muscle performance, balance and mobility: a randomised controlled trial [ISRCTN62535013]. BMC Geriatr 2005, 5:17.

35. Beck BR, Norling TL: The effect of 8 mos of twice-weekly low- or higher intensity whole body vibration on risk factors for postmenopausal hip fracture. Am J Phys Med Rehabil 2010, 89(12):997-1009.

36. Bogaerts A, Delecluse C, Boonen S, Claessens AL, Milisen K, Verschueren SM: Changes in balance, functional performance and fall risk following whole body vibration training and vitamin $\mathrm{D}$ supplementation in institutionalized elderly women. A 6 month randomized controlled trial. Gait Posture 2011, 33(3):466-472.

37. Bogaerts A, Delecluse C, Claessens AL, Coudyzer W, Boonen S, Verschueren SM: Impact of whole-body vibration training versus fitness training on muscle strength and muscle mass in older men: a 1-year randomized controlled trial. J Gerontol A Biol Sci Med Sci 2007, 62(6):630-635.

38. Bogaerts AC, Delecluse C, Claessens AL, Troosters T, Boonen S, Verschueren SM: Effects of whole body vibration training on cardiorespiratory fitness and muscle strength in older individuals (a 1-year randomised controlled trial). Age and ageing 2009, 38(4):448-454.

39. Calder CG, Mannion J, Metcalf PA: Lowintensity whole-body vibration training to reduce fall risk in active, elderly residents of a retirement village. J Am Geriatr Soc 2013, 61(8):1424-1426.

40. Furness TP, Maschette WE: Influence of whole body vibration platform frequency on neuromuscular performance of communitydwelling older adults. J Strength Cond Res
2009, 23(5):1508-1513.

41. Furness TP, Maschette WE, Lorenzen C, Naughton GA, Williams MD: Efficacy of a whole-body vibration intervention on functional performance of communitydwelling older adults. J Altern Complement Med 2010, 16(7):795-797.

42. Gomez-Cabello A, Gonzalez-Aguero A, Ara I, Casajus JA, Vicente-Rodriguez G: Effects of a short-term whole body vibration intervention on physical fitness in elderly people. Maturitas 2013.

43. Iwamoto J, Sato $\mathrm{Y}$, Takeda T, Matsumoto $\mathrm{H}$ : Whole body vibration exercise improves body balance and walking velocity in postmenopausal osteoporotic women treated with alendronate: Galileo and Alendronate I ntervention Trail (GAIT). J Musculoskelet Neuronal Interact 2012, 12(3):136-143.

44. Kemmler W, S VS, Mayer S, Niedermayer M, Hentschke C, Kalender WA: [Effect of whole body vibration on the neuromuscular performance of females 65 years and older. One-year results of the controlled randomized ELVIS study]. Z Gerontol Geriatr 2010, 43(2):125-132.

45. Kennis E, Verschueren SM, Bogaerts A, Coudyzer W, Boonen S, Delecluse C: Effects of fitness and vibration training on muscle quality: a 1-year postintervention follow-up in older men. Arch Phys Med Rehabil 2013, 94(5):910-918.

46. Klarner A, von Stengel S, Kemmler W, Kladny B, Kalender W: [Effects of two different types of whole body vibration on neuromuscular performance and body composition in postmenopausal women]. Dtsch Med Wochenschr 2011, 136(42):2133-2139. 
47. Lachane CC: The effects of whole-body vibration exercise on muscular strength in seniors. University of Windsor, Ontario, Canada; 2012.

48. Machado A, Garcia-Lopez D, GonzalezGallego J, Garatachea N: Whole-body vibration training increases muscle strength and mass in older women: a randomizedcontrolled trial. Scand J Med Sci Sports 2010, 20(2):200-207.

49. Mikhael M, Orr R, Amsen F, Greene D, Singh MA: Effect of standing posture during whole body vibration training on muscle morphology and function in older adults: a randomised controlled trial. BMC Geriatr 2010, 10:74.

50. Raimundo AM, Gusi N, Tomas-Carus $P$ : Fitness efficacy of vibratory exercise compared to walking in postmenopausal women. Eur J Appl Physiol 2009, 106(5):741-748.

51. Rees S, Murphy A, Watsford M: Effects of vibration exercise on muscle performance and mobility in an older population. J Aging Phys Act 2007, 15(4):367-381.

52. Rees S, Murphy A, Watsford ML: Effects of whole-body vibration exercise on lowerextremety muscle strength and power in an older population: a randomized clinical trial Physical therapy 2008, 88(462-470).

53. Roelants M, Delecluse C, Verschueren SM: Whole-body-vibration training increases knee-extension strength and speed of movement in older women. J Am Geriatr Soc 2004, 52(6):901-908.

54. Rogan S, Hilfiker R, Schmid S, Radlinger L: Stochastic resonance whole-body vibration training for chair rising performance on untrained elderly: a pilot study. Arch
Gerontol Geriatr 2012, 55(2):468-473.

55. Russo CR, Lauretani F, Bandinelli S, Bartali B, Cavazzini C, Guralnik JM, Ferrucci L: High-frequency vibration training increases muscle power in postmenopausal women. Arch Phys Med Rehabil 2003, 84(12):18541857.

56. Stolzenberg $\mathrm{N}$, Belavy $\mathrm{DL}$, Rawer $\mathrm{R}$, Felsenberg D: Vibration or balance training on neuromuscular performance in osteopenic women. Int J Sports Med 2013, 34(11):956-962.

57. Verschueren SM, Bogaerts A, Delecluse C, Claessens AL, Haentjens P, Vanderschueren D, Boonen S: The effects of whole-body vibration training and vitamin D supplementation on muscle strength, muscle mass, and bone density in institutionalized elderly women: a 6-month randomized, controlled trial. J Bone Miner Res 2011, 26(1):42-49.

58. Verschueren SM, Roelants M, Delecluse C, Swinnen S, Vanderschueren D, Boonen S: Effect of 6-month whole body vibration training on hip density, muscle strength, and postural control in postmenopausal women: a randomized controlled pilot study. J Bone Miner Res 2004, 19(3):352359.

59. von Stengel S, Kemmler W, Engelke K, Kalender WA: Effect of whole-body vibration on neuromuscular performance and body composition for females 65 years and older: a randomized-controlled trial. Scand J Med Sci Sports 2012, 22(1):119127.

60. Zhang L, Weng C, Liu M, Wang Q, Liu L, He $Y$ : Effect of whole-body vibration exercise on mobility, balance ability and general 
health status in frail elderly patients: a pilot randomized controlled trial. Clin Rehabil 2014, 28(1):59-68.

61. Sievanen $\mathrm{H}$, Karinkanta $\mathrm{S}$, Moisio-Vilenius P, Ripsaluoma J: Feasibility of whole-body vibration training in nursing home residents with low physical function: a pilot study. Aging Clin Exp Res 2014, 26(5):511-517.

62. Alvarez-Barbosa F, Del Pozo-Cruz J, Del Pozo-Cruz B, Alfonso-Rosa RM, Rogers ME, Zhang Y: Effects of supervised whole body vibration exercise on fall risk factors, functional dependence and health-related quality of life in nursing home residents aged 80+. Maturitas 2014, 79(4):456-463.

63. Osugi T, Iwamoto J, Yamazaki M, Takakuwa M: Effect of a combination of whole body vibration exercise and squat training on body balance, muscle power, and walking ability in the elderly. Ther Clin Risk Manag 2014, 10:131-138.

64. Sitja-Rabert M, Martinez-Zapata MJ, Fort Vanmeerhaeghe A, Rey Abella F, RomeroRodriguez D, Bonfill X: Effects of a Whole Body Vibration (WBV) Exercise Intervention for Institutionalized Older People: A Randomized, Multicentre, Parallel, Clinical Trial. Journal of the American Medical Directors Association 2014.

65. Corrie H, Brooke-Wavell $\mathrm{K}$, Mansfield NJ, Cowley A, Morris R, Masud T: Effects of vertical and side-alternating vibration training on fall risk factors and bone turnover in older people at risk of falls. Age and ageing 2015, 44(1):115-122.

66. Leung KS, Li CY, Tse YK, Choy TK, Leung PC, Hung VW, Chan SY, Leung AH, Cheung WH: Effects of 18-month low-magnitude high-frequency vibration on fall rate and fracture risks in 710 community elderly-a cluster-randomized controlled trial. Osteoporos Int 2014, 25(6):1785-1795.

67. Ochi A, Abe T, Yamada K, Ibuki S, Tateuchi $\mathrm{H}$, Ichihashi $\mathrm{N}$ : Effect of balance exercise in combination with whole-body vibration on muscle activity of the stepping limb during a forward fall in older women: A randomized controlled pilot study. Arch Gerontol Geriatr 2014.

68. Orr R: The effect of whole body vibration exposure on balance and functional mobility in older adults: A systematic review and meta-analysis. Maturitas 2015.

69. Caspersen CJ, Powell KE, Christenson GM: Physical activity, exercise, and physical fitness: definitions and distinctions for health-related research. Public Health Rep 1985, 100(2):126-131.

70. Medicine ACoS: ACSM's Guidelines for Exercise Testing and Prescription: ACSM (Lippincott Raven); 2013.

71. Forster A, Lambley R, Young JB: Is physical rehabilitation for older people in long-term care effective? Findings from a systematic review. Age and ageing 2010, 39(2):169175.

72. Campbell KL, Neil SE, Winters-Stone KM: Review of exercise studies in breast cancer survivors: attention to principles of exercise training. British journal of sports medicine 2012, 46(13):909-916.

73. Kawanabe K, Kawashima A, Sashimoto I, Takeda T, Sato Y, Iwamoto J: Effect of whole-body vibration exercise and muscle strengthening, balance, and walking exercises on walking ability in the elderly. The Keio journal of medicine 2007, 56(1):28-33. 
74. Osawa $\mathrm{Y}$, Oguma $\mathrm{Y}$, Ishii $\mathrm{N}$ : The effects of whole-body vibration on muscle strength and power: a meta-analysis. J Musculoskelet Neuronal Interact 2013, 13(3):380-390.

75. Cardinale M, Bosco C: The use of vibration as an exercise intervention. Exerc Sport Sci Rev 2003, 31(1):3-7.

76. Lord SR, Menz HB, Tiedemann A: A physiological profile approach to falls risk assessment and prevention. Physical therapy 2003, 83(3):237-252.

77. Pedrero-Chamizo R, Gomez-Cabello A, Delgado S, Rodriguez-Llarena S, Rodriguez-Marroyo JA, Cabanillas E, Melendez A, Vicente-Rodriguez G, Aznar $S$, Villa $G$ et al: Physical fitness levels among independent non-institutionalized Spanish elderly: the elderly EXERNET multicenter study. Arch Gerontol Geriatr 2012, 55(2):406-416. 


\section{Additional material}

\section{Additional file 1 Search terms in PubMed:}

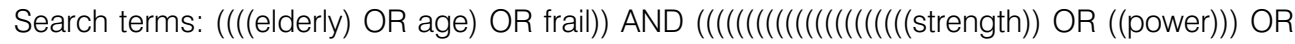
((rate of force development))) OR ((force))) OR ((functional strenght))) OR ((fall))) OR ((falls))) OR ((faller))) OR ((fallers))) OR ((sway))) OR ((postural reaction))) OR ((postural stability))) OR ((postural balance))) OR ((gait stability))) OR ((dynamic stability)))) OR ((propriocept*))) OR ((sensori-motor))) OR ((sensorimotor)))) AND ((("whole body vibration") OR ("whole body vibrations") OR (Vibration Training[tiab]) OR (stochastic stimulation) OR (stochastic training) OR (stochastic vibration) OR (Whole-Body Vibration) OR (Whole-Body-Vibration) OR (Whole-Body Vibrations) OR (Whole-Body-Vibrations) OR (WBV) OR (sinusoidal vibration) OR (noise vibration AND (therapy OR treatment OR training OR exercise)) OR (stochastic resonance therapy) OR (stochastic resonance treatment))) 


\section{Additional file 2}

Forest plot overview of Classification Go-Go, outcome: all strength outcomes (IMVC, DS, Power, RFD, FS)

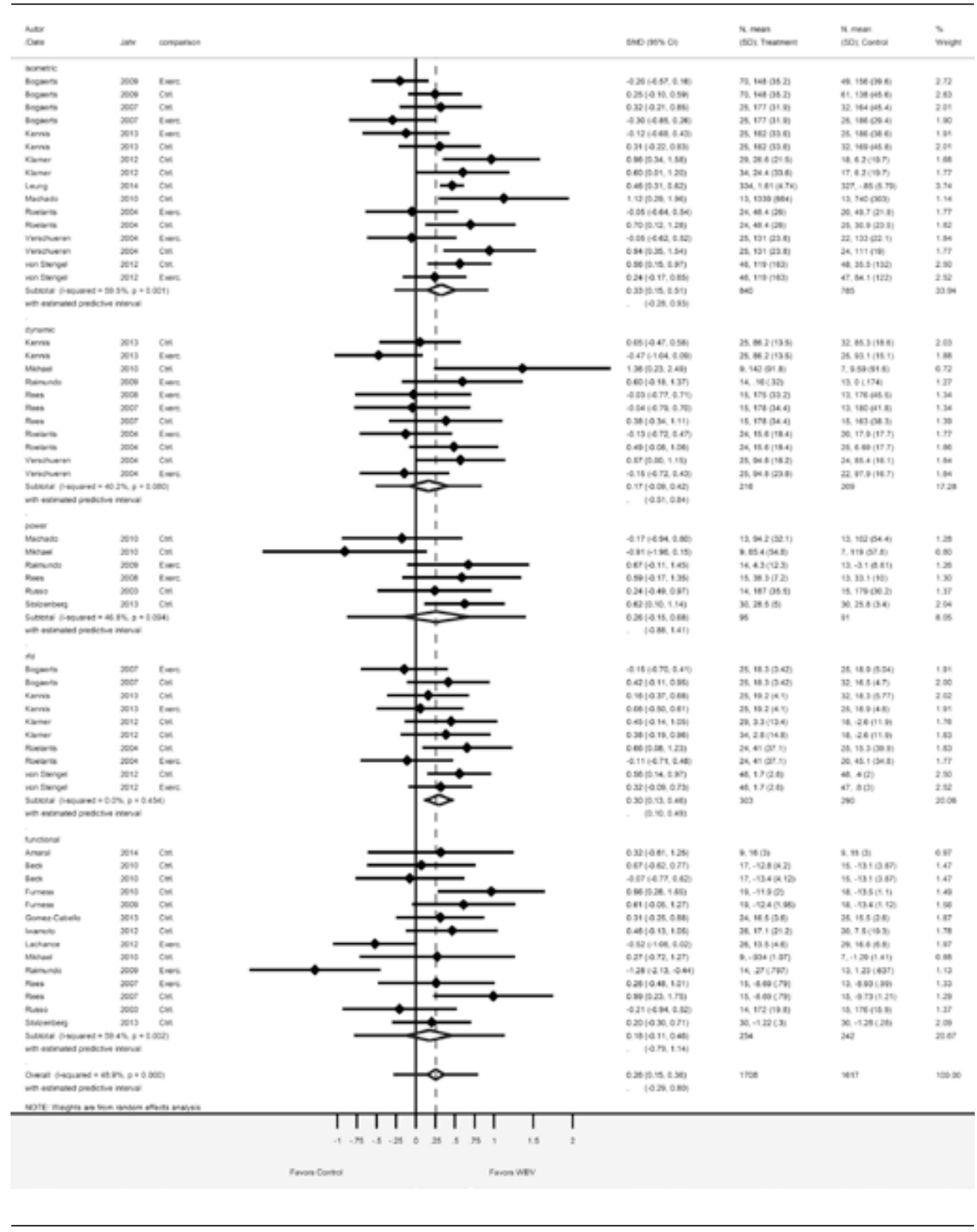




\section{Additional file 3}

Forest plot overview of Classification Slow-Go, outcome: all strength outcomes (IMVC, DS, Power, RFD, FS)

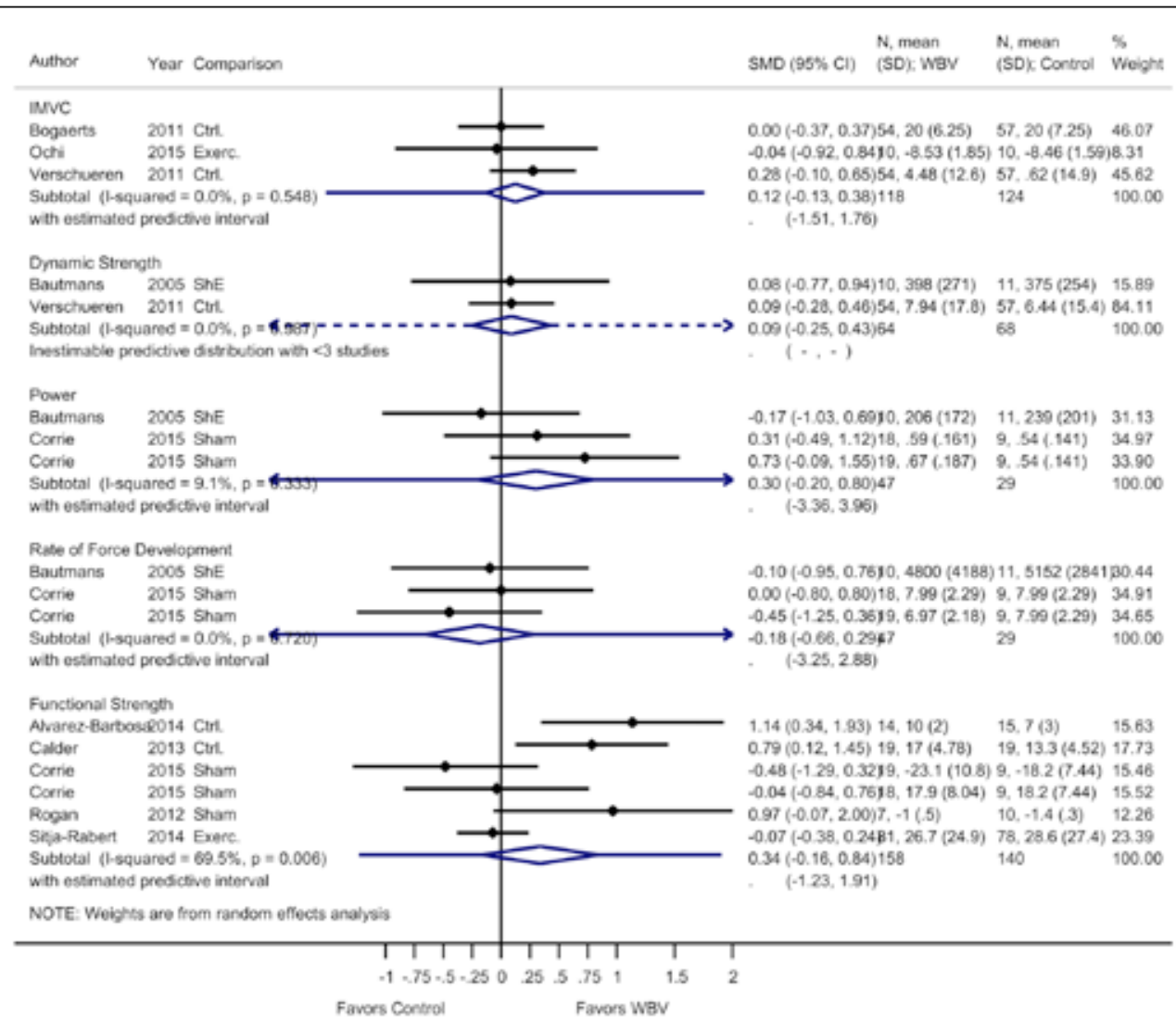




\section{Additional file 4}

Forest plot overview of Classification No-Go, outcome: all strength outcomes (IMVC, DS, Power, RFD, FS)

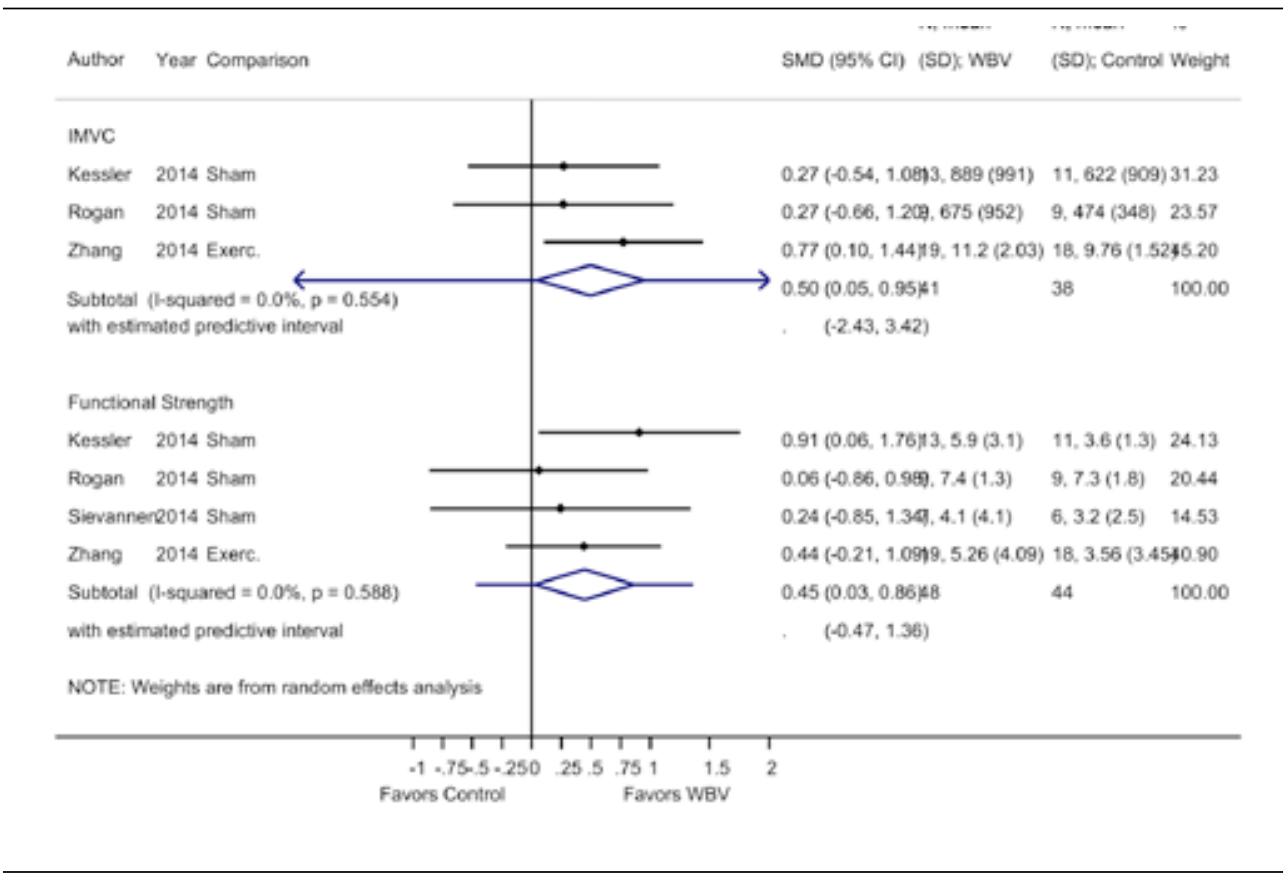





\title{
Chapter 4
}

\author{
"Skilling up" for training: a feasibility study \\ investigating acute effects of stochastic resonance \\ whole-body vibration on postural \\ control of older adults"
}

Ageing Research 2012, 4:e5

doi:10.4081/ar.2012.e5

Rogan S

Radlinger $\mathrm{L}$

Schmid S

Herren $\mathrm{K}$

Hilfiker $\mathrm{R}$

de Bruin ED 


\section{ABSTRACT}

\section{Background}

The present pilot study investigated the feasibility of applying a single bout stochastic resonance whole-body vibration in deconditioned elderly individuals and the effects on static and dynamic balance and reaction time.

\section{Methods}

A non-blinded randomized control trial with a pre-test/post-test design was used. Twenty elderly individuals were randomised into either SR-WBV $(n=10$, frequency $5 \mathrm{~Hz}$, Noise 4) or no-treatment control $(n=10)$. SR-WBV received 5 sets of 1 -minute stochastic whole-body vibration $(5 \mathrm{~Hz}$, Noise 4: vibration with a randomly varying frequency, $1=$ low and $4=$ high) with 1 minute 's rest in between. The control group rested for 10 minutes without any intervention. Functional reach test (FRT), semi-tandem stand (STS), Expanded Timed Get Up-and-Go Test (ETGUG), Single Task- (ST) and Dual Task Walking time (DT), chair rising (CR) and foot (RTF) and hand reaction time $(\mathrm{RTH})$ were measured before and after the intervention.

Within- and between group differences were analyzed using repeated measures. In order to assess the meaningfulness of pre-training to post-training changes, the effect size (ES) was calculated according Cohens $d$.

\section{Results}

All included participants accepted and adhered to the WBV session and performed scheduled follow-up measurements. There were no adverse events. Change values for dynamic balance showed a strong trend towards improvement for FRT of about $4.5 \% \quad(E S=0.52, p=0.161)$. Change values for RTF (5.9\%; $E S=0.55 ; p=0.169)$ showed a trend towards improvement in the SR-WBV only.

\section{Conclusion}

The results suggest that stochastic resonance WBV is both safe and well accepted by elderly individuals dwelling in assisted living institutions and might have beneficial effects on balance in these adults. Further research is warranted to determine whether this device might be of use in the "skilling up" phase of an exercise program when training is initiated in strongly deconditioned and/or frail elderly. The new training protocol is expected to allow for safe "skilling-up" training of deconditioned older adults in assisted living institutions.

ClinicalTrial.gov: NCT01045746 


\section{Background}

The balance abilities of ageing subjects become increasingly limited due to normal or pathological ageing [1]. These age related limitations in balance abilities may be explained by changes in muscle mass, decreased reflex activity, mobility impairments, loss of somatosensory sensors as well as being due to an impairment of central processing, a deficit of motor response functions and a reduction in the functioning of the vestibular and visual systems [2]. This implies that an observable limitation in balance abilities in the clinical setting is most probably multifactorial. These limitations are, furthermore, most likely associated with risk of injury or risk of falls $[3,4]$. Fall-related injuries such as hip fractures are considerable problems in elderly individuals, as they often lead to sustained disability. A sedentary lifestyle in older people increases the risk of falling whereas physically active older people have a clear reduced risk, especially for falls with injuries [5]. In summary these findings indicate that ways should be found to encourage elderly individuals to become more physically active.

It can be expected that an improvement in balance abilities through better postural control may lead to a decreased risk of falling. Therefore, preventive programs are needed in order to reduce disability, reduce the costs of health care and to improve the quality of life of elderly individuals. It is well known that exercise programs can be effective for fall prevention in elderly individuals [6, 7]. What seems to be lacking from this line of research is an appreciation of what exercises are best suited and most effective for specific subpopulations of elderly individuals, e.g. for people who have marked physical limitations because of frailty who want to start a training program. Such populations are advised to first enter a "skilling up" phase before more traditional forms of training exercises are implemented [4]. The question of what kinds of exercises are appropriate for "skilling up", however, needs to be answered [8]. With this insight trainers may prescribe balance exercises more effectively to frail and deconditioned elderly individuals with different physical activity backgrounds that have impairments in static and dynamic balance skills.

Sensorimotor and exercise training are traditional intervention methods in the field of physical therapy. Recently, whole body vibration techniques have been introduced as a new methodology for balance training. Although there is much variation in protocol design, the results of this form of training, as summarised in a recent systematic review, are encouraging in the sense that WBV may be a viable alternative to exercise training in improving balance and functional mobility among sedentary and frail elderly individuals [9]. To date, research on the effect of WBV in general, and stochastic resonance WBV [10] in particular, on postural control in elderly individuals is sparse. For stochastic resonance WBV research on the level of randomised control study design is inexistent [11]. Furthermore, those WBV studies that were performed in the past mainly focused on elderly individuals below the age of 75 years [11]. Because no study has applied stochastic resonance WBV into an intervention for elderly individuals there is uncertainty about the needed dose levels, the possible treatment effects and its variance. 
Modern exercise equipment may not always be suitable for elderly individuals, who might be concerned about the intensity of training sessions and may rather express to have a preference for more traditional therapy approaches [12]. The effect on humans of exposure to whole body vibration is believed to cause, furthermore, a range of problems under certain conditions. New treatments, therefore, usually have to go through a series of phases to test whether they are feasible, safe and effective [13]. It seems in this context justified to perform a pilot study to assess the feasibility of applying stochastic resonance WBV in an elderly population. Findings of such a study can inform a larger scale main Phase III study [13].

The aim of this pilot study was to perform a phase II trial according the model for complex interventions advocated by the British Medical Research Council [14] to test the effects of stochastic resonance WBV in sedentary elderly individuals. Our intervention targeted individuals who were living in a senior citizens hostel who are likely to have deconditioning due to a sedentary life style [15]. This pilot study aimed to (1) develop a WBV intervention based on principles of exercise physiology and to deliver it to the individuals living in the hostel, (2) evaluate the feasibility of the intervention process and the ability to recruit and retain elderly individuals for such an intervention, and (3) assess the acute effects of the intervention.

\section{Methods}

\section{Participants and randomisation}

The study involved two randomised groups in a pre-test/post-test design with no blinding of the participants and assessors to the contents of the SR-WBV training.

Twenty elderly individuals (ten male and ten female) living in a residential care setting participated in this study (Table 1). They were recruited by oral presentation and a local newspaper article between November 2009 and January 2010.

\section{Table 1.}

A summary description of the demographic variables of the groups.

\begin{tabular}{llll}
\hline & $\begin{array}{l}\text { SR-WBV } \\
(\mathbf{n = 1 0})\end{array}$ & $\begin{array}{l}\text { Control } \\
(\mathbf{n = 1 0})\end{array}$ & P-value \\
\hline Age (years) & $80.2 \pm 6.8$ & $77.4 \pm 7.1$ & 0.406 \\
Height (cm) & $167,1 \pm 6.8$ & $172.9 \pm 9.4$ & 0.173 \\
Weight (kg) & $74.4 \pm 2.8$ & $75.2 \pm 11.5$ & 1.0 \\
Gender female/male & $5 / 5$ & $5 / 5$ & 1.0 \\
BMl (Kg/m²) & $26.8 . \pm .4$ & $25 \pm 1.8$ & 0.199 \\
Falls Efficacy Scale-International (FES-l) & $19.6 \pm 7.3$ & $17.4 \pm 4.2$ & 0.330 \\
Amount of individuals using walking aids (\%) & $10 \%$ & $10 \%$ & 1.0 \\
\hline
\end{tabular}

Abbreviations: cm: centimeters; KG: kilogram; Kg/m²: kilogram/meter², SR-WBV: stochastic resonance whole-body vibration; \pm : standard deviation; \%: percent 
Participants were included if they were aged older than 65 years, were able to stand without aids, were classified as being lightly dependent on nursing care (BESA classification level 0 , $1,2^{1}$ [17]), living in Canton Bern and had a score $>22$ in the Mini-Mental Status Examination (MMSE). Exclusion criteria were: visual disturbances², lower or upper limb prostheses, acute joint diseases, acute thrombosis, acute fractures, acute infections, acute tissue damage, or acute surgical scars.

The randomization procedure was performed by an independent research assistant with Microsoft Excel 2010. The participants were stratified by sex and allocated to either an intervention (receiving SR-WBV) or a control group (receiving no intervention), using a computer generated sequence.

The participants were familiarized with the treatment protocol one week prior to data collection. The study protocol has been approved by the local Ethics Committee of Canton Bern (No. 228/09) and was registered under ClinicalTrial.gov: NCT01045746. All participants gave written informed consent.

\section{Criteria for success}

The criteria for success, an important part of a pilot study [13], were based on the primary feasibility objective and focused on recruitment, attrition and adherence to the stochastic resonance WBV exercise. Values for these parameters were compared with median rates in falls prevention interventions in institutional settings for clinical trials [18]. Recruitment of a third of the residents that are eligible for the training session, a $15 \%$ attrition rate, and $80 \%$ adherence to the WBV session were deemed acceptable.

For recruitment, data for the total sampling frame (both those approached and not approached) for inclusion in the trials were taken to assess generalizability to all elderly individuals within the facility. We measured the inclusion rate-i.e. the proportion of participants invited to participate who enrolled into the study - and distinguished between those who refused, did not respond or who were willing but excluded (volunteered but did not meet the study inclusion criteria).

For attrition, we measured the number of participants lost at final follow-up.

For adherence to the intervention we recorded engagement with the intervention, e.g. compliance with all 5 consecutive times of 1 minute SR-WBV with a 1 minute rest in between the sets.

\section{Secondary outcome measurements}

Semi-Tandem Stand (STS) was measured using a multi-component force platform (Kistler, Typ 9286BA, Winterthur, Switzerland). The participants were instructed to perform a semi-tandem stand (STS) for 20 seconds on the force platform. They were positioned by placing their right foot

1 BESA and its assessment instruments are based on the results of scientific research. Thanks to the four steps of BESA - clarification of the available resources, agreements concerning the aims of health care, taxation of the costs and improvement of the quality level of health care - the main elements of the process of health care are systematically sustained. BESA is actually used in more than 400 homes and residences for elderly people in Switzerland.

2 Visual disturbances are abnormalities of sight and associated with neurological disorders (diabetes), often include double vision, moving vision like nystagmus, blindness or reduced view field. 
in the right upper quadrant and the left foot in the left lower quadrant of a custom built calibre that was removed after positioning. The arms were in a neutral position on the side at the body. The participants had to look straight ahead and fix their gaze on a green marker positioned at eye-level at a distance of 3 meters. Anterior-posterior (AP) and medial-lateral (ML) sway during the STS was measured. The test was repeated twice with a rest of 1 minute in between.

The Functional Reach Test (FRT) was used for measuring dynamic balance [19]. This is a validated clinical measure for balance [20]. The FRT has been associated with an increased risk of falls and frailty in the elderly who are unable to reach more than $15 \mathrm{~cm}$ [19]. Five measures were recorded on each side and a mean score from three out of five was calculated.

Chair rising (CR) was used as a performance measurement of upper leg strength and was performed on a multi-component force platform (Kistler, Typ 9286BA, Winterthur, Switzerland). Participants were required to rise from a chair as quickly as possible, to recover balance and to stand upright as fast as possible for 20 seconds. For standing, the participants were instructed to look straight ahead and to fix their gaze on a marker positioned at eye-level at a distance of 3 meters. The arms were crossed on the subject's chest during the whole testing procedure. Vertical ground reaction forces (VGRF) during chair rising were measured before and after intervention. Variables used were Chair rising time (CRT) and time to stabilization (TTS), normalized to body weight [21]. The test was repeated twice with a rest of 1 minute in between.

The Expanded Timed Get Up-and-Go (ETGUG) was used for measuring dynamic balance. The ETGUG [22] has good reliability for experienced raters and acceptable internal consistency. For the current study, the ETGUG was repeated twice.

Walking was assessed with different cognitive tasks [23] in single and dual task test conditions. For the single task condition (ST), the participants were instructed to walk a distance of 20 meters at their self-selected walking speed. For the dual task condition (DT), the participants had to count backwards from the number 250 in steps of 7 while walking the same distance at their self-selected walking speed. Total walking times were measured using a digital hand stopwatch (Timex: Ironmen Triathlon, Middlebury, CL, USA). The test was repeated twice.

Reaction time (RT) was measured from the hand (RTH) and feet (RTF) as these measures represent important systems involved in the maintenance of postural stability [24]. A finger and a foot push-button were used as the response to a random light stimulus. The seated participants placed their dominant hand on a table as well as their dominant foot in front of the push-button. The system measured the elapsed time from the release of the light stimulus to the moment the button was pressed. Participants performed 5 practice and 10 experimental trials. The best attempt of the 10 trials was recorded. 


\section{SR-WBV intervention}

The participants in the SR-WBV group were exposed to SR-WBV (Setting: $5 \mathrm{~Hz}$, Noise 4) using a Zeptor med ${ }^{\circledR}$ vibration device (Frei Swiss AG, Zürich, Switzerland). While standing on the platform of the vibration device, participants had to maintain an upright standing position with slightly flexed hip, knee and ankle joints. The treatment protocol consisted of 5 times of 1 minute SR-WBV with a 1-minute rest in between the sets. The participants in the control group were tested twice with a 10-minute break between tests.

Immediately following the vibration-treatment and the resting period, post-tests were carried out using the same protocol as for the pre-tests.

\section{Statistical analyses}

Descriptive statistics (Mann-Whitney U test) were used to assess baseline characteristics between SR-WBV and the control group. Group differences on STS (AP, ML), FRT, CRT, TTS, ETGUG, ST, DT, RTH and RTF were analyzed with a repeated measures ANOVA. The significance level adopted was $p<0.05$ a priori. All values are presented as means \pm standard deviations (SD) as well as 95\% confidence intervals. The mean differences between pre-test / post-test variables were calculated. The magnitude of difference between conditions was evaluated using Cohen's $d$ effect size (ES), where values $<0.20$ were considered trivial, 0.20 to 0.50 small, 0.50 to 0.80 moderate and $>0.80$ large effects [25]. All analyses were conducted using SPSS Version 18.0 (SPSS Inc., Chicago, IL, USA). The program G*Power 3 was used for the post hoc calculation of power (www.psycho.uni-duesseldorf.de/abteilungen/aap/gpower3/). The CONSORT 2010 guidelines regarding randomized trials (www.consort-statement.org) and recommendations of items to include when reporting a pilot study [13] were followed for describing the results of this pilot. 


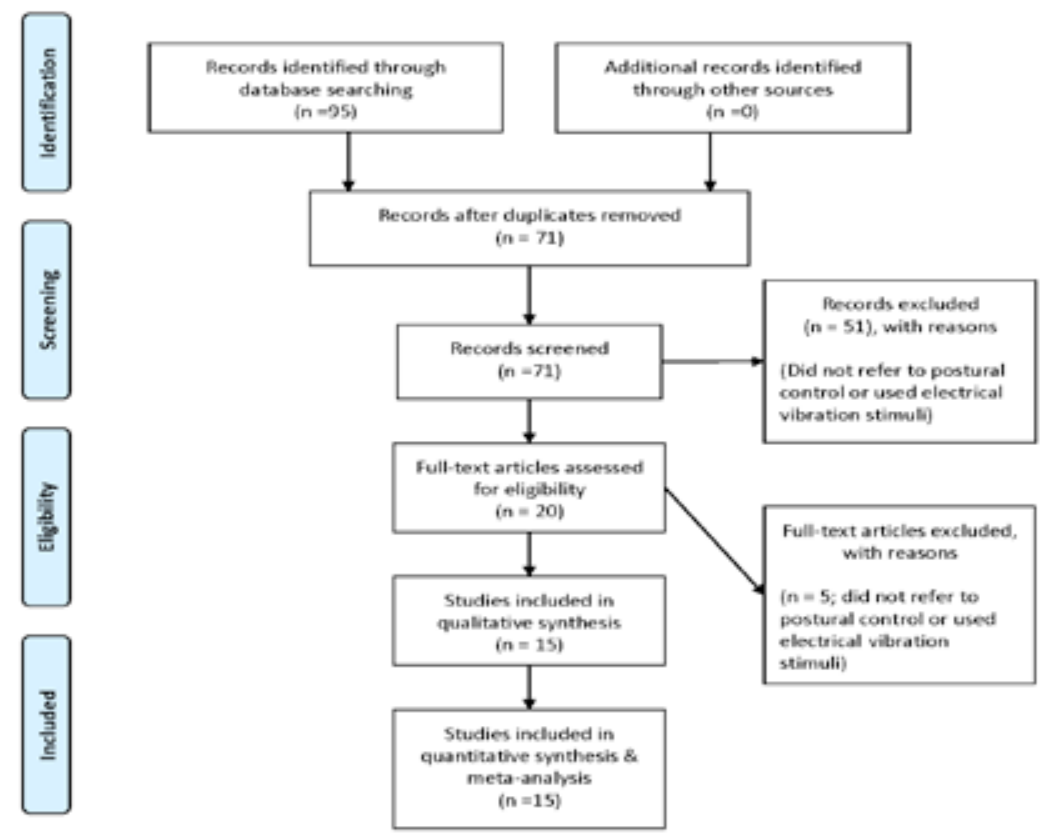

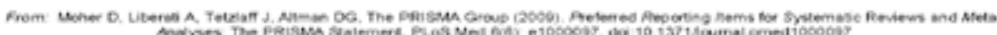
For more information, whit waw serisma-statement. ore.

Figure 1.

Flow chart for the trial 


\section{Results}

\section{Feasibility and Safety; recruitment, attrition and adherence}

The facility had a total of 100 residents from which staff representatives estimated sixty-five fulfilled eligibility criteria. Two information sessions were held and visited by 45 residents. Twentythree residents were not eligible and excluded. Twenty-two residents were eligible to participate based on the inclusion-exclusion criteria and invited to participate. From these twenty individuals consented to partake in the study. This resulted in a recruitment rate of $31 \%$ for the total sample frame. The inclusion rate - twenty-two invited to participate; twenty enrolled - was $91 \%$. One person declined to participate and one person had other reasons not to participate.

All elderly individuals participated at follow-up measurements which resulted in a $0 \%$ attrition rate. For adherence to the intervention we had 100\% compliance with all 5 consecutive times of 1 minute SR-WBV with a 1-minute rest in between the sets. Figure 1 summarizes the flow of participants through the study per training group.

The participants were willing to be randomized. Neither subjective nor objective side-effects related to the used intervention were reported (Fig. 1). Socio-demographic and anthropometric characteristics of the two groups are summarized in Table 1. At baseline and after intervention, no statistically significant differences $(p<0.05)$ were found between groups.

Table 2 shows the effect of SR-WBV on the secondary outcomes between both groups. For the outcome STS no significant difference was found. Only for RTF a significant difference was determined $\left(F_{1,18}=9.277 ; p=0.007\right)$.

Within-group shows no significant results on primary and secondary outcome measures (Results not shown). 
Table 2

Results for static and dynamic balance and reaction time between-group

\begin{tabular}{|c|c|c|c|}
\hline & $\begin{array}{l}\text { SR-WBV group } \\
\text { Mean } \pm \text { SD }\end{array}$ & $\begin{array}{l}\text { Control group } \\
\text { Mean } \pm \text { SD }\end{array}$ & p-value; [95\% Cl]; ES \\
\hline \multicolumn{4}{|c|}{ STSML (mm) } \\
\hline pre & $31.67 \pm 9.3$ & $38.70 \pm 12.3$ & $0.207 ;[26-47.2]$ \\
\hline post & $34.79 \pm 11.1$ & $40.60 \pm 14.2$ & 0.092 \\
\hline \multicolumn{4}{|c|}{ STSAP $(\mathrm{mm})$} \\
\hline pre & $27.02 \pm 9.5$ & $35.7 \pm 15$ & $0.86 ;[19.39-64.11]$ \\
\hline post & $27.92 \pm 5.7$ & $39.5 \pm 18.7$ & 0.163 \\
\hline \multicolumn{4}{|c|}{ FRT $(\mathrm{cm})$} \\
\hline pre & $27.78 \pm 8.6$ & $31.89 \pm 6.8$ & 0.299; [26.6 - 33.9] \\
\hline post & $29.02 \pm 8.1$ & $32.36 \pm 8.0$ & 0.06 \\
\hline \multicolumn{4}{|c|}{ CR (s) } \\
\hline pre & $1.40 \pm 0.19$ & $1.36 \pm 0.20$ & $0.858 ;[1.26-1.51]$ \\
\hline post & $1.35 \pm 0.17$ & $1.35 \pm 0.17$ & 0.002 \\
\hline \multicolumn{4}{|c|}{ TTS (s) } \\
\hline pre & $0.48 \pm 0.32$ & $0.48 \pm 0.29$ & $0.610 ;[0.28-0.62]$ \\
\hline post & $0.37 \pm 0.17$ & $0.47 \pm 0.28$ & 0.017 \\
\hline \multicolumn{4}{|c|}{ ETGUG 0-2 m (s) } \\
\hline pre & $3.38 \pm 0.8$ & $2.97 \pm 0.8$ & $0.173 ;[2.8-3.6]$ \\
\hline post & $3.46 \pm 1.3$ & $3.03 \pm 0.8$ & 0.60 \\
\hline \multicolumn{4}{|c|}{ ETGUG 2-8 m (s) } \\
\hline pre & $5.40 \pm 2.24$ & $4.12 \pm 0.95$ & $0.147 ;[3.9-5.5]$ \\
\hline post & $5.19 \pm 2.24$ & $4.19 \pm 0.81$ & 0.113 \\
\hline \multicolumn{4}{|c|}{ ETGUG turn (s) } \\
\hline pre & $3.74 \pm 1.45$ & $3.29 \pm 0.95$ & $0.378 ;[2.9-4]$ \\
\hline post & $3.62 \pm 1.34$ & $3.15 \pm 0.74$ & 0.43 \\
\hline \multicolumn{4}{|c|}{ ETGUG 12-18 m (s) } \\
\hline pre & $5.27 \pm 2.01$ & $4.01 \pm 0.95$ & $0.97[3.8-5.3]$ \\
\hline post & $5.08 \pm 2.02$ & $3.93 \pm 0.84$ & 0.145 \\
\hline \multicolumn{4}{|c|}{ ETGUG 18-20 m (s) } \\
\hline pre & $2.33 \pm 0.78$ & $2.15 \pm 0.69$ & $0.280[1.9-2.5]$ \\
\hline post & $2.38 \pm 0.81$ & $1.93 \pm 0.39$ & 0.64 \\
\hline \multicolumn{4}{|l|}{ ST (s) } \\
\hline pre & $17.33 \pm 6.52$ & $15.06 \pm 3.87$ & $0.638[13.8-18.6]$ \\
\hline post & $16.17 \pm 6.28$ & $15.20 \pm 4.04$ & 0.13 \\
\hline \multicolumn{4}{|c|}{ DT (s) } \\
\hline pre & $21.01 \pm 9.38$ & $17.89 \pm 4.81$ & $0.442[15.5-22.2]$ \\
\hline post & $19.13 \pm 8.84$ & $17.26 \pm 4.16$ & 0.33 \\
\hline \multicolumn{4}{|c|}{ RTH (s) } \\
\hline pre & $0.307 \pm 0.08$ & $0.250 \pm 0.04$ & $0.096[0.259-0.314]$ \\
\hline post & $0.312 \pm 0.07$ & $0.276 \pm 0.06$ & 0.146 \\
\hline \multicolumn{4}{|c|}{ RTF (s) } \\
\hline pre & $0.359 \pm 0.07$ & $0.271 \pm 0.04$ & $0.07 ;[0.294-0.341]$ \\
\hline post & $0.343 \pm 0.06$ & $0.296 \pm 0.05$ & 0.40 \\
\hline
\end{tabular}

Values are in mean $( \pm \mathrm{SD})$ for each intervention. P-values after pre and post intervention were computed using a Wilcoxon signed ranks test for SR $(n=10)$ and CT $(n=10)$. Effect size $(E S)$ computed with $\mathrm{G}^{\star}$ Power $3.95 \% \mathrm{Cl}=95 \%$ confidence interval. * Statistically significant $(P \leq 0.05)$ after pre and post intervention. Abbreviations: STSML: Semi-Tandem Stand medio-lateral; STSAP: Semi-Tandem Stand anterior-posterior; FRT: Functional Reach Test; CR: chair rising; TTS: time to stabilization; ETGUG: Expanded Timed Get Up-and-Go; ST: single task; DT: dual task; RTH: reaction time hand; RTF: reaction time feet; $\mathrm{cm}$ : centimeter; m: meter; mm: millimeter; s: seconds 


\section{Discussion}

The aim of the current study was to (1) develop a WBV intervention based on principles of exercise physiology and to deliver it to the individuals living in the hostel, (2) evaluate the feasibility of the intervention process and the ability to recruit and retain elderly individuals for such an intervention, and (3) assess the acute effects of the intervention.

We demonstrated the feasibility of acquiring acceptable recruitment, attrition and adherence rates for elderly individuals in an assisted living facility randomized to the experimental WBV training arm. Our target of about a third recruitment rate for the total sample frame was only closely met. Those individuals that responded and visited an information session showed a large inclusion rate. These findings indicate the importance of information sessions for elderly individuals where questions and concerns about new interventions can be met.

Compliance with the WBV intervention and retesting was excellent. All initially included twenty elderly individuals completed the training and retest data were obtained from all twenty elderly individuals. This is far more than the rate that could have been expected. It should be noted, however, that the mean compliance rate that was previously determined for several studies summarized in a systematic review [18] mainly focused on studies with far longer time periods. Our data should, therefore, be replicated in another study where the intervention is applied during several training sessions over several weeks. It can be expected hat because of such a longitudinal design in an institutional setting less favorable compliance and retention rates are to be expected. Our findings warrant, however, such follow-up studies based on these first results. Safety and acceptance are critical features of the program and we applied strict exclusion criteria for this small cohort, particularly as it was a pilot study. It is hoped that the criteria can be relaxed in time as experience is gained.

Neither did the elderly individuals of the SR-WBV nor did those who were acting as controls show improvements or tendencies towards improvement in any of the outcome parameters within a bout of 5 consecutive one-minute single SR-WBV training sessions. Kawanabe and colleagues [6] indicated that acute effects of WBV training in the elderly may be expected after a two months' study period.

Summarizing the findings and limitations of this study it becomes clear that this study only reveals first estimates for the chosen outcome measures. We implemented a strict study design to control threats to validity. Should the protocol continue to be successful we envisage stochastic resonance WBV will be undertaken as skilling-up exercise in frail deconditioned older people. Prior to this, further formal evaluation of potential risks and outcome is required in longitudinal studies. A next step would be to replicate the findings in a new exercise group of frail elderly individuals as an additional control procedure [17]. We are therefore undertaking an RCT to rigorously compare the WBV protocol with the current standard of usual care. The data collected allow for calculation of the sample size needed for a larger study. The FRT test, for example, showed that the baseline means were not different and the effect size of the intervention was 
moderate at 0.52 . To avoid a type I or II error in a future study we need, based on our observed values for the FRT (with values post-training of: SR-WBV $=30.13 \pm 8.02$; Control $=33.3 \pm 8.02$ ), an estimated sample size of 120 participants for a two group RCT-design. This would result in $80 \%$ power at a-level 0.05 and is based on the assumption that the standard deviation of the response variable is 8.02 . The sample size would be around 106 participants when the foot reaction time is taken as the primary outcome. With this in mind, the relationship between WBV research and its effect on postural control in frail elderly individuals requires further exploration. Translating the results from postural control experiments with non-frail elderly to therapeutic interventions should, therefore, take place with caution until the appropriate clinical studies with clinically relevant population outcomes have been conducted. In frail elderly the FRT will be around $15 \mathrm{~cm}$ [26]. This implies that the possible range for improvements due to training will be much larger which in turn will affect the sample size needed to obtain sufficient power in a future study design.

The lack of blinding of both participants and assessors is a very major limitation of this pilot study that should be different in a future phase III trial. It should be possible to offer a 'sham' vibration procedure that is able to successfully blind the participants and, thus, overcomes many of the potential placebo effects. Likewise, it seems possible to blind the future assessor(s) to the intervention.

\section{Acknowledgements}

This study was funded by the Bern University of Applied Science, Health (aR\&D Physiotherapy). We thank all residents of the Senevita Residenz Multengut Muri (Senevita AG, Wabern, Switzerland) for their participation in this study. The funding agency played no role in the design, conduct, or reporting of the study or in the decision to submit the article for publication. 


\section{References}

1. Lacour M, Bernard-Demanze L, Dumitrescu

M: Posture control, aging, and attention resources: models and posture-analysis methods. Neurophysiol Clin 2008, 38(6):411-421.

2. Goble DJ, Coxon JP, Wenderoth N, Van Impe A, Swinnen SP: Proprioceptive sensibility in the elderly: degeneration, functional consequences and plasticadaptive processes. Neurosci Biobehav Rev 2009, 33(3):271-278.

3. Pizzigalli L, Filippini A, Ahmaidi S, Jullien $H$, Rainoldi A: Prevention of falling risk in elderly people: the relevance of muscular strength and symmetry of lower limbs in postural stability. J Strength Cond Res 2011, 25(2):567-574.

4. Skelton D, Dinan SM: Exercise for falls management: Rationale for an exercise programme aimed at reducing postural instability. Physiotherapy Theory and Practice 1999, 15(2):105-120.

5. Thibaud M, Bloch F, Tournoux-Facon C, Brèque C, Rigaud AS, Dugué B, Kemoun G: Impact of physical activity and sedentary behaviour on fall risks in older people: a systematic review and meta-analysis of observational studies. Eur Rev Aging Phys Act 2011, 8(1).

6. Kawanabe K, Kawashima A, Sashimoto I, Takeda T, Sato Y, Iwamoto J: Effect of whole-body vibration exercise and muscle strengthening, balance, and walking exercises on walking ability in the elderly. Keio J Med 2007, 56(1):28-33.

7. Bogaerts A, Verschueren S, Delecluse C, Claessens AL, Boonen S: Effects of whole body vibration training on postural control in older individuals: a 1 year randomized controlled trial. Gait Posture 2007, 26(2):309-316.

8. Landi F, Abbatecola AM, Provinciali M, Corsonello A, Bustacchini S, Manigrasso L, Cherubini A, Bernabei R, Lattanzio F: Moving against frailty: does physical activity matter? Biogerontology 2010, 11(5):537545.

9. Merriman $\mathrm{H}$, Jackson $\mathrm{K}$ : The effects of whole-body vibration training in aging adults: a systematic review. J Geriatr Phys Ther 2009, 32(3):134-145.

10. Srygley JM, Mirelman A, Herman T, Giladi N, Hausdorff JM: When does walking alter thinking? Age and task associated findings. Brain Res 2009, 1253:92-99.

11. Rogan S, Hilfiker R, Herren $\mathrm{K}$, Radlinger L, de Bruin ED: Effects of whole-body vibration on postural control in elderly: a systematic review and meta-analysis. BMC Geriatr 2011, 11(1):72

12. Laver K, Ratcliffe J, George S, Burgess L, Crotty M: Is the Nintendo Wii Fit really acceptable to older people?: a discrete choice experiment. BMC Geriatr 2011, $11: 64$.

13. Thabane L, Ma J, Chu R, Cheng J, Ismaila A, Rios LP, Robson R, Thabane M, Giangregorio L, Goldsmith $\mathrm{CH}$ : A tutorial on pilot studies: the what, why and how. BMC Medical Research Methodology 2010, 10(1):1471-

14. Craig P, Dieppe P, Macintyre S, Michie S, Nazareth I, Petticrew M: Developing and evaluating complex interventions: the new 
Medical Research Council guidance. Brit Med J 2008, 337(7676).

15. McPhee B, GARY Foster G, Long A: Bad Vibrations- A Handbook on whole-body vibration exposure in mining, 2 edn. Sydney, Australia; 2009.

16. Kollensperger M, Geser F, Seppi K, Stampfer-Kountchev M, Sawires M, Scherfler C, Boesch S, Mueller J, Koukouni V, Quinn $N$ et al: Red flags for multiple system atrophy. Mov Disord 2008, 23(8):1093-1099.

17. Peter-Egger G: Assessmentsinstrumente von BESA und ihre Auswirkungen auf die Pflege. Pflegewissenschaft 2007

18. Nyman SR, Victor CR: Older people's recruitment, sustained participation, and adherence to falls prevention interventions in institutional settings: a supplement to the Cochrane systematic review. Age Ageing 2011, 40(4):430-436.

19. Duncan PW, Weiner DK, Chandler J, Studenski S: Functional reach: a new clinical measure of balance. J Gerontol 1990, 45(6):M192-197.

20. Weiner DK, Duncan PW, Chandler J, Studenski SA: Functional reach: a marker of physical frailty. J Am Geriatr Soc 1992, 40(3):203-207.

21. Wikstrom EA, Tillman MD, Smith AN, Borsa PA: A new force-plate technology measure of dynamic postural stability: The dynamic postural stability index. Journal of Athletic Training 2005, 40(4):305-309.

22. Botolfsen P, Helbostad JL, Moe-Nilssen R, Wall JC: Reliability and concurrent validity of the Expanded Timed Up-and-Go test in older people with impaired mobility. Physiother Res Int 2008, 13(2):94-106.

23. Beauchet $\mathrm{O}$, Annweiler $\mathrm{C}$, Dubost $\mathrm{V}$, Allali G, Kressig RW, Bridenbaugh S, Berrut G,
Assal F, Herrmann FR: Stops walking when talking: a predictor of falls in older adults? Eur J Neurol 2009, 16(7):786-795.

24. Lord SR, Castell S, Corcoran J, Dayhew J, Matters B, Shan A, Williams P: The effect of group exercise on physical functioning and falls in frail older people living in retirement villages: a randomized, controlled trial. J Am Geriatr Soc 2003, 51(12):1685-1692.

25. Ross SE, Guskiewicz KM: Time to stabilization: A method for analyzing dynamic postural stability. Athletic Therapy Today 2003, 8(3):37-39.

26. Jonsson E, Henriksson M, Hirschfeld $\mathrm{H}$ : Does the functional reach test reflect stability limits in elderly people? Journal of rehabilitation medicine 2003, 35(1):26-30. 



\section{Chapter 5}

Feasibility and effects of applying stochastic resonance whole-body vibration on untrained elderly:

A randomized crossover pilot study 


\section{ABSTRACT}

\section{Background}

Aging is associated with loss of balance and activity in daily life. It impacts postural control and increases the risk of falls. The current study was conducted to determine the feasibility and longterm impact of stochastic resonance whole-body vibration (SR-WBV) on static and dynamic balance and reaction time among elderly individuals.

\section{Methods}

A randomized crossover pilot study with blinding of the participants. Twenty elderly were divided into group A (SR-WBV $5 \mathrm{~Hz}$, Noise 4 / SR-WBV $1 \mathrm{~Hz}$, Noise 1) or group B (SR-WBV $1 \mathrm{~Hz}$, Noise 1 / SR-WBV $5 \mathrm{~Hz}$, Noise 1). Feasibility outcomes included recruitment, compliance and safety. Secondary outcomes were Semi-Tandem Stand (STS), Functional Reach Test (FRT), Expanded Timed Get Up-and-Go (ETGUG), walking under single (ST) \& dual task (DT) conditions, hand and foot reaction time (RTH / RTF). Puri and Sen Rank-Order L Statistics were used to analyze carry-over effects. To analyze SR-WBV effects Wilcoxon signed-ranked tests were used.

\section{Results}

With good recruitment rate (55\%) and compliance (attrition 15\%; adherence $85 \%$ ) rates the intervention was deemed feasible. Three participants dropped out, two due to knee pain and one for personal reasons. ETGUG 0 to $2 \mathrm{~m}$ ( $p=0.143$; ES: 0.36 ) and ETGUG total time ( $p=$ 0.097; ES: 0.40) showed medium effect sizes.

\section{Conclusions}

Stochastic resonance training is feasible in untrained elderly resulting in good recruitment and compliance. Low volume SR-WBV exercises over 12 training sessions with $5 \mathrm{~Hz}$, Noise 4 seems a sufficient stimulus to improve ETGUG total time. The stimulation did not elicit changes in other outcomes.

\section{Trial registration}

This trial has been registered at the U.S. National Institutes of Health under NCT01045746 (https://clinicaltrials.gov/ct2/show/NCT01045746). 


\section{Background}

Postural balance skills of the elderly become increasingly limited due to normal or pathological ageing [1]. These age related limitations in balance skills may be explained by changes in muscle mass, decreased reflex activity, mobility impairments, loss of somatosensory sensors as well as being due to an impairment of central processing, a deficit of motor response functions and a reduction in the functioning of the vestibular and visual systems [2,3]. These limitations are, furthermore, most likely associated with risk of injury or risk of falls [4-6]. A sedentary lifestyle in elderly individuals further increases the risk of falling whereas physically active elderly have a reduced risk, especially for falls resulting in injuries [7].

Despite the fact that physical activity (PA) for elderly is one of the major elements for general health prevention, too few elderly engage in PA [8]. Inactive or sedentary elderly should, therefore, be motivated to increase their PA [9]. It is important, however, to consider low baseline fitness and mobility levels in pre-frail or frail or rather untrained elderly when starting an exercise program. Elderly individuals with low baseline fitness and mobility levels who want to start a training program should start with an exercise program that meets their physical capabilities [10]. These individuals are advised to first enter a "skilling up" phase before more traditional forms of training are implemented [5] in case their capabilities are low. The question of what kinds of exercises are appropriate for "skilling up", however, remain to be explored [11]. With this insight, trainers may prescribe balance exercises more effectively for untrained and frail elderly with different physical activity backgrounds who have impairments in static, dynamic or functional balance skills.

Systematic reviews concluded that, compared to more demanding interventions, whole body vibration (WBV) might be a more safe and less-fatiguing type of exercise [12] with beneficial effects on dynamic balance skills [13]. Pilot studies showed that an intervention with stochastic resonance WBV (SR-WBV) in the elderly is both safe and feasible [14] and has positive effects on physical functioning [15]. The use of SR-WBV might, therefore, be valuable for untrained or frail elderly where the neuromuscular systems might not be able withstanding higher loading and long training sessions [16].

There are two types of WBV devices on the market [13]: sinusoidal (i.e. with a constant vibration frequency) and stochastic resonance vibration (i.e. with random vibration frequencies and harmonics) [17]. During sinusoidal WBV participants stand on a single plate platform that vertically or side alternating vibrates with high frequency. Frequencies range between 20 to $50 \mathrm{~Hz}$ and amplitudes between 2 to $14 \mathrm{~mm}$. Stochastic resonance whole-body vibration (SRWBV) devices vibrate with frequencies between 1 and $12 \mathrm{~Hz}$ and amplitudes between 3 and 6 $\mathrm{mm}$ while the feet of the participants are placed on two independently powered stochastically resonating vibrating platforms [13].

However, there is a lack of evidence concerning the feasibility of implementing such exercise interventions in a primary training program aimed at "skilling up" of untrained elderly. New treatments usually have to go through a series of phases to test whether they are safe and 
effective [18] before larger scale studies and application in clinical practice are to be considered. The aim of this pilot study was to perform a phase II trial according the model for complex interventions advocated by the British Medical Research Council [19] to test the feasibility and effects of a SR-WBV program in a group of untrained elderly. The study aimed to (1) develop an exercise intervention based on principles of exercise theory and to deliver it to untrained elderly, (2) evaluate the feasibility of the intervention and the ability to recruit and retain elderly individuals, and (3) assess whether the treatment had some effect on physical performance.

\section{Methods}

\section{Design}

The study used a crossover research design, involving 2 randomized groups (groups A and B) with blinding of the participants (Figure 1). The SR-WBV exercise was supervised and performed in a home-for-the-aged (Senevita Residenz Multengut, Muri, Switzerland), however, focused on individuals comparable with older community-dwellers independent in mobility functions and not on individuals comparable to nursing home residents. Group A started with the intervention while group B received a sham intervention. Treatments were reversed for the groups following a wash-out period. The assessor was not blinded to group allocation. The participants were familiarized with the treatment protocol one week prior to data collection.

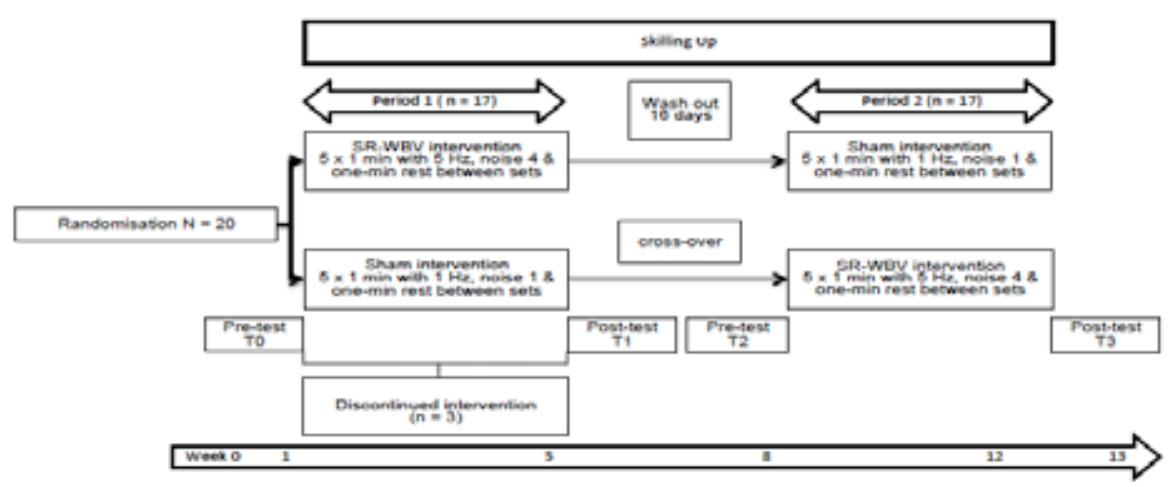

\section{Figure 1}

Flow chart of this cross-over pilot study. 


\section{Participants}

Participants were included when fulfilling the following criteria: age over 65 years, able to stand with or without walking aids, classified as being only lightly dependent on nursing care according the BESA classification level 0, 1, 2a, living in the Canton of Berne and a having a score $>22$ in the Mini-Mental Status Examination (MMSE) Test. Exclusion criteria were: visual disturbances ${ }^{b}$, lower or upper leg prosthesis, acute joint disease, acute thrombosis, acute fractures, acute infections, acute tissue damage, acute surgical scars or alcohol abuse.

\section{Randomization}

Randomization was performed by an independent research assistant. The participants were stratified by sex and were randomly assigned to either group A or group B. The blinded independent research assistant guaranteed concealed allocation sequence through the use of numbered sealed opaque envelopes distributed after the completion of all baseline assessments. Written consent was obtained from all participants before enrolment in the study. The study protocol was approved by the Ethical Committee of Canton Berne (No.228/09) and was based on the declaration of Helsinki and registered under the U.S. National Institutes of Health (http:// clinicaltrial.org) trial Number NCT01045746.

\section{Protocol}

The participants were exposed to SR-WBV using a Zeptor med® device (Frei Swiss AG, Zurich, Switzerland) containing two three dimensionally vibrating plates (Figure 2). The participants were familiarized with the vibration treatment one week prior to the experiment. They stood freely on both legs wearing comfortable shoes. The participants were instructed to maintain a standing position with slight flexion of the hips, knees and ankle joints. In period 1, the participants in group A received 5 sets of 1 minute SR-WBV with $5 \mathrm{~Hz}$, Noise 4 with 1 minute of rest between sets, three times a week, during four weeks. A minimum of one day rest in between training sessions was warranted. Participants in group B received a sham intervention of 5 sets of 1 minute SR-WBV with $1 \mathrm{~Hz}$, Noise 1 , where the $1 \mathrm{~Hz}$ frequency condition can be expected to have no training effect $[20,21]$. After a wash-out period of 16 days, period 2 started. Group A received the sham intervention of 5 sets of 1 minute SR-WBV with $1 \mathrm{~Hz}$, Noise 1 and group B received 5 sets of 1 minute SR-WBV with $5 \mathrm{~Hz}$ and Noise 4 during four weeks. The primary and secondary outcome variables were measured at baseline (T0) before training, after four weeks of training (T1) in period 1, and after the second four weeks' intervention period (T3). 


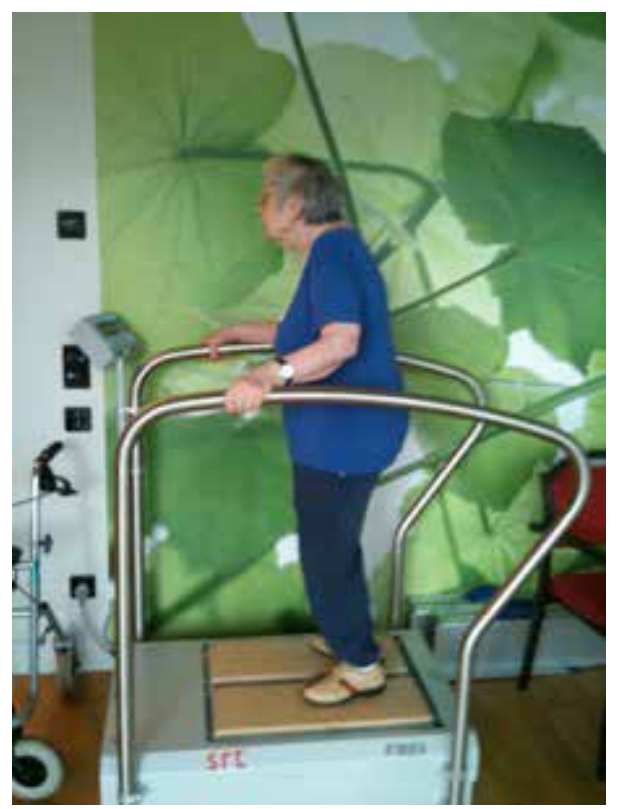

\section{Figure 2.}

Picture of the Zeptor med® device.

\section{Outcomes}

\section{Recruitment rate, attrition, and program adherence}

The criteria for success [18] of this pilot study were based on feasibility and focused on recruitment, attrition and adherence to the stochastic resonance WBV intervention. Recruitment of a third of the residents deemed eligible for the training, a 15\% attrition rate, and $80 \%$ attendance rate [22] for the training were deemed acceptable.

For recruitment, data for the total sampling frame (both those approached and not approached) for inclusion in the trial were taken to assess generalizability to all elderly individuals within the facility. We measured the inclusion rate, i.e. the proportion of participants invited to participate who enrolled into the study, and distinguished between those who refused, did not respond or who were willing but excluded (volunteered but did not meet the study inclusion criteria). For attrition, we measured the number of participants lost at follow-up. For adherence to the intervention we recorded engagement with the intervention, e.g. compliance with all trainings. There were a total of 24 stochastic resonance WBV training sessions possible for each individual. 


\section{Secondary outcomes}

Semi-Tandem Stand (STS) was measured using a multi-component force platform (Kistler, Typ 9286BA, Winterthur, Switzerland). STS measurement with this approach has shown to be reliable [23]. Each platform signal was transformed in an amplifier, digitally sampled with $1 \mathrm{kHz}$ using a 12-bitA-/D-converter (Meilhaus, ME-2600i, SisNova, Engeneering, Zug, Switzerland) and analyzed using ADS-Software-Program 1.12 (uk-labs, Kempten, Germany). The participants were instructed to perform STS for 20 seconds on the force platform. They were positioned by placing their right foot in the right upper quadrant and the left foot in the left lower quadrant of a custom built cadre that was removed after positioning. The arms were in a neutral position at the side of the body. The participants had to look straight ahead and fix their focal viewpoint on a green marker positioned at eye-level at a distance of 3 meters. Anterior-posterior (AP) and medial-lateral (ML) sway during the STS was calculated from force-time curves. The test was repeated twice with a rest of 1 minute in between.

Functional Reach Test (FRT) was used to quantify dynamic balance [24]. This test is both valid and reliable [25] for the target population. A yardstick is attached to a wall at about shoulderheight. The participant stands facing the wall, with the arm in $90^{\circ}$ anteflexion, and reaches maximally forward without moving the feet. The FRT has been associated with an increased risk of falls and frailty in elderly unable to reach more than $15 \mathrm{~cm}$ forward [24]. The measurement data were obtained from the best three attempts out of five and averaged.

The Expanded Timed Get Up-and-Go (ETGUG), a reliable clinical assessment, measured time series of functionally important tasks using a multimemory stopwatch $[26,27]$. The participants were asked to rise from a chair, to walk at their normal speed to the end of the walkway, to turn around and to walk and sit back on the chair. At 2, 8 and 10 meters along the walkway, markers were set (using colored tape on the floor), allowing measurement of the mean times. Measurements were conducted using a digital hand stopwatch (Timex: Ironmen Triathlon, Middlebury, CL, USA). The test was repeated twice with minimum of one-minute rest in between. Gait of each participant was assessed during usual walking at preferred velocity under single and dual task conditions over a distance of $20 \mathrm{~m}$ in a corridor of the Senevita Residenz Multengut Muri (Switzerland) with a digital hand stopwatch (Timex: Ironmen Triathlon, Middlebury, CL, USA). The test was repeated twice with a minimum of one-minute rest in between. Gait assessment at preferred velocity informs about actual, subject-specific behavior [28] which is indicative of a decrease in the performance of gait in senior adults who have fallen and may be related to diminished strength, balance, and tactile sensation [29]. The measurement device and testing protocol have previously been described and identified as being reliable in older adults [30].

Simple reaction time was measured from both hand (RTH) and foot (RTF) to measure psychomotor speed in milliseconds using a hand-held electronic timer and a light as the stimulus and depression of a switch by the finger and the foot as the responses [31] [32]. Participants performed 5 practice and 10 experimental trials.

For safety reasons, the participants were interviewed before and immediately after vibration 
training on their well-being, feelings of (in)stability and for adverse effects such as dizziness and pain during vibration. Discontinuations of participation in the study were noted.

\section{Statistical analysis}

This pilot study used nonparametric statistical analyses. Mann-Whitney $U$ test assess baseline characteristics (T0) and treatment effects between group A and group B. Puri and Sen L Statistics for Ranked Data [33] analyzed carry-over effects. Carry-over effects were compared with a twofactorial analysis of variance with repeated measures [34]. Pillai's Trace was used to calculate L. In case a carry-over effect was present, the data were analyzed with the first period data only: e.g., similar to a parallel design [34]. In case no carry-over effect was present, treatment effects were calculated with Mann-Whitney $U$ test from both periods. The difference in mean from period 1 minus period 2 was compared between the groups [35].

The results are reported as an estimate of the intervention effect as mean \pm SDs. P-values $<0.05$ were considered significant. All analyses were conducted using SPSS Version 19.0 for Windows (SPSS Inc., Chicago, IL, USA) and the statistical function of the Microsoft ${ }^{\circledR}$ Excel ${ }^{\circledR} 2008$ for Mac Version12.2.7 software. The magnitude of effects were calculated and expressed as $r=Z / \sqrt{ } N$. For $r$ an effect size of 0.1 is considered a "small" effect, around 0.3 a "medium" effect and 0.5 and above, a 'large' effect [36]. In addition, participants' compliance to the treatment protocol was calculated using the following formula: Number of vibration sessions $\div$ the total number of possible vibration sessions $\times 100$.

The program G*Power 3 was used for the post hoc calculation of power (www.psycho. uni-duesseldorf.de/abteilungen/aap/gpower3/). The CONSORT 2010 guidelines regarding randomized trials (www.consort-statement.org) and recommendations of items to include when reporting a pilot study [37] were followed for describing the results of this pilot.

Results

Fig. 1 describes the flow of the participants through the study. Socio-demographic and anthropometric characteristics are summarized in Table 1. None of the participants reported any injuries or medical conditions that could affect their balance. Participants reported to being generally healthy. 
Table 1

Demographic characteristics and baseline values (mean $\pm \mathrm{SD})$

\begin{tabular}{llll}
\hline & $\begin{array}{l}\text { Group A } \\
\text { (n= 10) }\end{array}$ & $\begin{array}{l}\text { Group B } \\
\text { (n= 10) }\end{array}$ & p \\
\hline Age (years) & $76.8 \pm 7.7$ & $80.7 \pm 5.7$ & 0.290 \\
Height (m) & $1.76 \pm 0.07$ & $1.64 \pm 0.05$ & 0.001 \\
Weight (kg) & $81.00 \pm 10.4$ & $69.20 \pm 9.8$ & 0.034 \\
BMI (Kg/cm $)$ & $26.1 \pm 2.5$ & $25.8 \pm 3.8$ & 0.597 \\
Sway AP (mm) & $36.5 \pm 6.7$ & $30.1 \pm 12.0$ & 0.162 \\
Sway ML (mm) & $32.9 \pm 9.1$ & $26.8 \pm 15.0$ & 0.199 \\
FRT (cm) & $33.2 \pm 7.2$ & $28.3 \pm 8.1$ & 0.174 \\
ETGUG ss (s) & $2.20 \pm 1.0$ & $2.72 \pm 1.3$ & 0.226 \\
ETGUG 0-2 m (s) & $1.67 \pm 0.7$ & $2.05 \pm 1.2$ & 0.472 \\
ETGUG 2-8 m (s) & $3.46 \pm 0.9$ & $3.69 \pm 2.2$ & 0.364 \\
ETGUG turn (s) & $3.15 \pm 0.9$ & $3.87 \pm 1.4$ & 0.151 \\
ETGUG 12-18 m (s) & $4.10 \pm 0.9$ & $5.18 \pm 2.1$ & 0.121 \\
ETGUG 18-20 m (s) & $2.20 \pm 0.8$ & $2.19 \pm 0.8$ & 0.112 \\
ETGUG total time (s) & $16.8 \pm 3.4$ & $20.1 \pm 7.0$ & 0.096 \\
ST (m/s) & $0.77 \pm 0.2$ & $0.88 \pm 0.3$ & 0.705 \\
DT (m/s) & $0.85 \pm 0.3$ & $1.1 \pm 0.4$ & 0.082 \\
RTH (ms) & $276 \pm 0.7$ & $281 \pm 0.7$ & 0.791 \\
RTF (ms) & $299 \pm 0.6$ & $331 \pm 0.8$ & 0.545 \\
\hline
\end{tabular}

Abbreviations: AP: anterior-posterior, ML: medial-lateral, FRT: Functional Reach Test, ETGUG: Expanded Timed Get Up-andGo, ST: single task, DT: dual task, RTH: reaction time hand, RTF: reaction time foot, mm: millimeter, s: seconds, m/s: meter/ seconds, ms: milliseconds, ss: sit-to-stand, m: meter.

\section{Recruitment, attrition, and adherence}

The facility had a total of 100 residents from which staff representatives estimated 65 fulfilled eligibility criteria and, therefore, represented the potential sampling frame. Two information sessions were held and attended by 45 residents. From these 45 persons, 25 persons were deemed eligible and were invited to participate. 20 eligible persons (10 women, $79.85 \pm 6.6$ years and 10 men, $78 \pm 7.3$ years) were recruited and enrolled in the study resulting in a recruitment rate of approximately $55 \%$. Inclusion rate-i.e. the proportion of participants invited to participate who enrolled-was $80 \%$. The participants were willing to be randomized. Seventeen elderly individuals participated at follow-up measurements that resulted in a 15\% attrition rate. Three participants dropped out during the training sessions. Two participants discontinued training due to knee pain unrelated to the training and one for personal reasons (Table 2). The number of SR-WBV sessions completed divided by the possible training sessions was $95 \%$, leading to excellent adherence to the study protocol over the four weeks training periods. Neither subjective nor objective side-effects related to the used intervention were reported. 


\section{Table 2.}

Overview for repeated measures Puri \& Sen-analyses of ranked data for cross-over effect

\begin{tabular}{|c|c|c|c|}
\hline & Pillai's trace $\left(r^{2}=S_{B_{\text {Bet }}} / S_{\text {Tot }}\right)$ & $\mathbf{L}\left[(\mathbf{N}-1) \mathbf{r}^{2}\right]$ & Probability \\
\hline Sway AP (mm) & 0.038 & 0.278 & 0.761 \\
\hline Sway ML (mm) & 0.044 & 0.321 & 0.731 \\
\hline $\mathrm{FRT}(\mathrm{cm})$ & 0.255 & 2.394 & 0.128 \\
\hline ETGUG (s) ss & 0.291 & 2.877 & 0.090 \\
\hline ETGUG (s) 0-2 m & 0.062 & 0.467 & 0.637 \\
\hline ETGUG (s) 2-8 m & 0.175 & 1.485 & 0.260 \\
\hline ETGUG (s) turn & 0.009 & 0.066 & 0.936 \\
\hline ETGUG (s) 12-18 m & 0.239 & 2.201 & 0.148 \\
\hline ETGUG (s) 18-20 m & 0.064 & 0.482 & 0.628 \\
\hline ETGUG (s) total time & 0.273 & 2.626 & 0.108 \\
\hline $\mathrm{ST}(\mathrm{m} / \mathrm{s})$ & 0.156 & 1.295 & 0.305 \\
\hline DT (m/s) & 0.158 & 1.134 & 0.300 \\
\hline RTH (ms) & 0.022 & 0.159 & 0.855 \\
\hline RTF (ms) & 0.271 & 2.607 & 0.109 \\
\hline
\end{tabular}

Abbreviations: AP: anterior-posterior, ML: medial-lateral, FRT: Functional Reach Test, ETGUG: Expanded Timed Get Up-andGo, ST: single task, DT: dual task, RTH: reaction time hand, RTF: reaction time foot, mm: millimeter, s: seconds, m/s: meter/ seconds, ms: milliseconds, ss: sit-to-stand, m: meter.

\section{Secondary outcomes}

No carry-over effect was found for any of the outcomes measures (Table 2). Table 3 summarizes the main outcome results for all outcome measures. ETGUG 0 to $2 \mathrm{~m}(\mathrm{p}=0.143$; ES: 0.36$)$ and ETGUG total time ( $p=0.097$; ES: 0.40 ) showed no significant changes albeit medium effect sizes. The other values presented no significant changes combined with small effect sizes. 
Table 3.

Difference values from group $A$ and $B$ in mean $\pm S D$

\begin{tabular}{|c|c|c|c|c|}
\hline & $\begin{array}{l}\text { Group A } \\
\text { Difference period } 1 \text { - } 2\end{array}$ & $\begin{array}{l}\text { Group B } \\
\text { Difference period } 2 \text { - } 1\end{array}$ & $\mathbf{P}$ & ES \\
\hline Sway ML (mm) & $-11.73 \pm 4.6$ & $-10.23 \pm 6.3$ & 0.435 & 0.00 \\
\hline Sway AP (mm) & $-6.91 \pm 4.5$ & $-11.79 \pm 5.6$ & 1.000 & 0.19 \\
\hline FRT (cm) & $-12.67 \pm 14.6$ & $-2.33 \pm 0.8$ & 0.432 & 0.19 \\
\hline ETGUG (s) ss & $1.29 \pm 0.5$ & $1.39 \pm 0.3$ & 0.770 & 0.06 \\
\hline ETGUG (s) 0-2 m & $-0.39 \pm 0.7$ & $-0.07 \pm 0.2$ & 0.143 & 0.36 \\
\hline ETGUG (s) 2-8 m & $-0.54 \pm 0.2$ & $-0.99 \pm 0.4$ & 0.435 & 0.19 \\
\hline ETGUG (s) turn & $-0.14 \pm 0.1$ & $0.04 \pm 0.2$ & 0.626 & 0.12 \\
\hline ETGUG (s) 12-18 m & $-0.20 \pm 0.2$ & $0.02 \pm 0.2$ & 0.495 & 0.17 \\
\hline ETGUG (s) $18-20$ m & $-0.20 \pm 0.1$ & $-0.23 \pm 0.2$ & 0.696 & 0.09 \\
\hline ETGUG total time (s) & $-0.13 \pm 0.3$ & $1.15 \pm 0.4$ & 0.097 & 0.40 \\
\hline $\mathrm{ST}(\mathrm{m} / \mathrm{s})$ & $-0.02 \pm 0.02$ & $-0.01 \pm 0.2$ & 1.000 & 0.00 \\
\hline DT $(\mathrm{m} / \mathrm{s})$ & $-0.02 \pm 0.01$ & $0.02 \pm 0.02$ & 0.329 & 0.24 \\
\hline RTH (ms) & $-0.008 \pm 0.009$ & $-0.005 \pm 0.01$ & 1.000 & 0.00 \\
\hline RTF (ms) & $-0.010 \pm 0.005$ & $-0.010 \pm 0.01$ & 0.329 & 0.24 \\
\hline
\end{tabular}

Abbreviations: Difference values from period 1 - period 2. P-values were computed using Wilcoxon signed rank test for group 1 and group 2 at period 1 and period 2 .

ES: effect size, AP: anterior-posterior, ML: medial-lateral, FRT: Functional Reach Test, ETGUG: Expanded Timed Get Up-andGo, ST: single task, DT: dual task, RTH: reaction time hand, RTF: reaction time foot, mm: millimeter, s: seconds, m/s: meter/ seconds, ms: milliseconds, ss: sit-to-stand, m: meter.

\section{Discussion}

This randomized cross-over pilot study tested the feasibility of SR-WBV training applied to untrained elderly living in a home-for-the-aged. Furthermore, this study investigated the effects of a four-week SR-WBV training on static and dynamic balance and reaction time. The main findings showed that a randomised controlled cross-over trial with SR-WBV is both feasible and safe for untrained elderly. Those individuals that responded and visited an information session showed a large inclusion rate and in majority remained in the intervention until completion. These findings indicate the importance of information sessions for elderly individuals where questions and concerns about new interventions can be met.

This pilot study provided useful information about the feasibility of the experimental intervention that used SR-WBV for "skilling-up" training. Our participants tolerated the SR-WBV intervention. They were also able to progress in intensity and duration of the exercises. However, our experience suggests that our SR-WBV component was not of sufficient duration and/or intensity to ameliorate physical functioning capacity as indicated by no improvements in the secondary outcomes. Neither group showed improvements or tendencies towards improvement in any of the outcome parameters within the program duration of four weeks. This finding might be 
attributed to a lack of power due to the sample size used or is due to the time frame of the intervention. Kawanabe and colleagues [38] indicated that effects of WBV training in the elderly may be expected after a two months study period. We believe, therefore, that it is feasible to proceed to a sufficiently powered main study only with major modifications to the protocol; e.g. adapt the intensities and/or length of training. It might well be, for example, that we should increase the frequency of the SR-WBV vibration. The current pilot study used a frequency with 5 $\mathrm{Hz}$, Noise 4. Haas [39-42] and Turbanski [43] used in their studies effective frequencies of $6 \mathrm{~Hz}$. In a future study, the amplitude of vibration should possibly be $6 \mathrm{~Hz}$.

Summarizing the findings and limitations of this study it becomes clear that this study only reveals first estimates for the chosen outcome measures. We implemented a strict study design to control threats to validity. A next step would be to replicate the findings in a new exercise group of institutionalized elderly individuals as an additional control procedure [44]. Although we are aware of the fact that the emphasis of a pilot study should be placed on feasibility and not on statistical significance [18] our data allow for a sample size calculation for a future trial. To avoid a type I or II error in this future trial we need, based on our observed value for the ETGUG total time (with values of the last training of EXPERIMENTAL $=13.5 \pm 3.1 \mathrm{~s}$; CONTROL $=19.3 \pm 7.9 \mathrm{~s}$ ), an estimated sample size of 50 participants per group for a two group pretest-posttest design. This would result in $80 \%$ power at an -level of 0.05 and is based on the assumption that the standard deviation of the response variable is 5.5. To account for attrition over time, the required sample size should increase by $15 \%$. It should be stressed, however, that this sample size calculation should be interpreted with caution because our estimates may be unrealistic or biased because of the limited sample size [18].

\section{Conclusions}

We conclude that pilot studies with explicit feasibility objectives and success criteria are important foundation steps in preparing for large trials [18] and for development of Rehabilitation research programs [45]. Ongoing formal review of the multifaceted issues inherent in the design and conduct of pilot studies can provide invaluable feasibility and scientific data for rehabilitation specialists, e.g. physiotherapists, willing to perform clinical trials [46] and may also be highly relevant for furthering the development of theory based rehabilitation [45]. SR-WBV training is feasible and, although not showing significant effects, shows trends to stronger improvement in the overall time to complete a series of functionally important tasks as assessed with the ETGUG with a medium to large effect size of 0.4. The application in a main study is deemed feasible, however, with a need for protocol modifications. A minimum of \pm 55 participants per group are required to achieve a power of $80 \%$ at the $5 \%$ level of significance based on ETGUG total time and considering the expectable attrition rate in a required larger scale study. This study encourages the further development of this intervention, preferably with a randomized control design. 


\section{Endnotes}

${ }^{a} B E S A$ and its assessment instruments are based on the results of scientific research. Thanks to the four steps of BESA - clarification of the available resources, agreements concerning the aims of health care, taxation of the costs and improvement of the quality level of health care - the main elements of the process of health are systematically sustained. BESA is actually used in more than 400 homes or residences for elderly people in Switzerland.

bVisual disturbances are abnormalities of sight and associated with neurological disorders (diabetes), often include double vision, moving vision like nystagmus, blindness or reduced view field.

\section{Authors' contributions}

SR, LR and DS conceived the idea of this study. SR, LR, RH, DS and EdB participated in the conception and design of the study; $\mathrm{RH}$ managed the randomising. $\mathrm{SR}$ and $\mathrm{RH}$ supervised the WBV trainings session and Data collection. SR conducted the statistical analyses. SR, LR, $\mathrm{RH}$ and $\mathrm{EdB}$ wrote the manuscript. SR, $\mathrm{LR}, \mathrm{RH}, \mathrm{DS}, \mathrm{RdB}$, and $\mathrm{EdB}$ were involved in drafting or revising the manuscript. All authors read and approved the final manuscript.

\section{Acknowledgement}

This study project was funded by the Bern University of Applied Sciences, Department Health (aR\&D Physiotherapy) and Senevita Residenz Multengut, Muri. Both institutions covered costs for insurance fees, ethical committee assessment and advertisement in the newspaper. The funding agency played no role in the design, conduct, or reporting of the study or in the decision to submit the article for publication. We would like to thank Ross Bennie for proof reading. 


\section{References}

1. Lacour M, Bernard-Demanze L, Dumitrescu M. Posture control, aging, and attention resources: models and postureanalysis methods. Neurophysiol Clin. 2008;38(6):411-21.

2. Goble DJ et al. Proprioceptive sensibility in the elderly: degeneration, functional consequences and plastic-adaptive processes. Neurosci Biobehav Rev 2009;33(3):271-8.

3. Rosso AL et al. Aging, the central nervous system, and mobility. J Gerontol A Biol Sci Med Sci. 2013;68(11):1379-86.

4. Pizzigalli $L$ et al. Prevention of falling risk in elderly people: the relevance of muscular strength and symmetry of lower limbs in postural stability. J Strength Cond Res. 2011;25(2):567-74.

5. Skelton DA, Dinan SM. Exercise for falls management: Rationale for an exercise programme aimed at reducing postural instability. Physiother Theory Pract 1999;15(2):105-20.

6. Tinetti ME, Speechley M, Ginter SF. Risk factors for falls among elderly persons living in the community. $\mathrm{N}$ Engl $\mathrm{J}$ Med. 1988;319(26):1701-7.

7. Thibaud $\mathrm{M}$ et al. Impact of physical activity and sedentary behaviour on fall risks in older people: a systematic review and meta-analysis of observational studies. Eur Rev Aging Phys Act. 2011;8:1.

8. Baert $V$ et al. Motivators and barriers for physical activity in the oldest old: a systematic review. Ageing Res Rev. 2011;10(4):464-74.

9. Freiberger E, Sieber C, Pfeifer K. Physical activity, exercise, and sarcopenia - future challenges. Wien Med Wochenschr. 2011;161(17-18):416-25

10. Gardner MM et al. Practical implementation of an exercise-based falls prevention programme. Age Ageing. 2001;30(1):7783.

11. Landi $F$ et al. Moving against frailty: does physical activity matter? Biogerontolgy. 2010;11(5):537-45

12. Chanou $\mathrm{K}$ et al. Whole-Body Vibration and Rehabilitation of Chronic Diseases: A Review of the Literature. J Sports Sci Med. 2012;11(2):187-200

13. Rogan $\mathrm{S}$ et al. Effects of whole-body vibration on postural control in elderly: a systematic review and meta-analysis. BMC Geriatr. 2011;11:72.

14. Rogan S; Schmidtbleicher D, Radlinger L Immediate effects after stochastic resonance whole-body vibration on physical performance on frail elderly for skilling-up training: a blind cross-over randomized pilot study. Aging Clin Exp Res. 2014; [Epub ahead of print].

15. Rogan $\mathrm{S}$ et al. Stochastic resonance whole-body vibration training for chair rising performance on untrained elderly: a pilot study. Arch Gerontol Geriatr. 2012;55(2):468-73

16. Herren $\mathrm{K}$ et al. Cardiovascular and metabolic strain during stochastic resonance therapy in stroke patients. Physioscience. 2009;5:13-7

17. Rogan S, Hilfiker R. [Training methods - increase muscle strength due to wholebody vibration - force with $\mathrm{Hz}$ ]. Sportverletz Sportschaden. 2012;26(4):185-7.

18. Thabane $L$ et al. A tutorial on pilot studies: 
the what, why and how. BMC Med Res Methodol. 2010;10:1.

19. Craig $P$ et al. Developing and evaluating complex interventions: the new Medical Research Council guidance. BMJ. 2008;337:a1655.

20. Rogan $S$ et al. Skilling up for training: a feasibility study investigating acute effects of stochastic resonance whole-body vibration on postural control of older adults. Ageing Res. 2012;4:e5.

21. Madou KH, Cronin JB. The effects of whole body vibration on physical and physiological capability in special populations. Hong Kong Physio J. 2008;26:24-38.

22. van Tulder $\mathrm{M}$ et al. Updated Method Guidelines for Systematic Reviews in the Cochrane Collaboration Back Review Group. Spine. 2003;28(12):1290-9. Doi: 10.1097/01.BRS.0000065484.95996.AF.

23. Ritchie $\mathrm{C}$ et al. Reliability and validity of physical fitness field tests for adults aged 55 to 70 years. J Sci Med Sport. 2005;8(1):6170.

24. Duncan PW et al. Functional reach: a new clinical measure of balance. J Gerontol. 1990;45(6):M192-7.

25. Weiner DK et al. Functional reach: a marker of physical frailty. J Am Geriatr Soc. 1992;40(3):203-7.

26. Wall JC et al. The Timed Get-up-and-Go test revisited: measurement of the component tasks. J Rehabil Res Dev. 2000;37(1):10913.

27. Botolfsen $\mathrm{P}$ et al. Reliability and concurrent validity of the Expanded Timed Up-and-Go test in older people with impaired mobility. Physiother Res Int. 2008;13(2):94-106.

28. Hamacher $D$ et al. Kinematic measures for assessing gait stability in elderly individuals: a systematic review. J R Soc Interface. 2011;8(65):1682-98.

29. Nelson AJ et al. The functional ambulation performance of elderly fallers and nonfallers walking at their preferred velocity. NeuroRehabilitation. 1999;13(3):141-6.

30. Bohannon RW. Comfortable and maximum walking speed of adults aged $20-79$ years: reference values and determinants. Age Ageing. 1997;26(1):15-9.

31. Lord SR, Castell S. Physical activity program for older persons: effect on balance, strength, neuromuscular control, and reaction time. Arch Phys Med Rehabil. 1994;75(6):648-52.

32. Lord SR, Clark RD, Webster IW. Postural stability and associated physiological factors in a population of aged persons. $J$ Gerontol. 1991;46(3):M69-76.

33. Thomas JR, Nelson JK, Thomas KT. A generalized rank-order method for nonparametric analysis of data from exercise science: a tutorial. Res Q Exerc Sport. 1999;70(1):11-23.

34. Cleophas, T.J., et al. Statistics applied to clinical trials. 5th ed2012: Springer.

35. Hills M, Armitage P. The two-period crossover clinical trial. $\mathrm{Br} \mathrm{J}$ Clin Pharmacol. 1979;8(1):7-20.

36. Corder GW, Foreman DI. Nonparameztric statistics for non-statisticans . a step-bystep approach. Hoboken, New Jersey: John Wiley \& Sons. Inc; 2009.

37. Thabane $L$ et al. A tutorial on pilot studies: the what, why and how. BMC Med Res Methodol. 2010;10(1):1471.

38. Kawanabe $\mathrm{K}$ et al. Effect of wholebody vibration exercise and muscle strengthening, balance, and walking exercises on walking ability in the elderly. 
Keio J Med. 2007;56(1):28-33.

39. Haas CT et al. The effects of random wholebody-vibration on motor symptoms in Parkinson's disease. NeuroRehabilitation. 2006;21(1):29-36.

40. Haas CT, Schmidtbleicher D. Neurorehabilitation via mechanischer Schwingungsreize, in DMGP Kongress. 2005.

41. Haas CT, Schmidtbleicher D. Potential of Stochastic Resonance in Neurorehabilitation. Isokinetic and Exercise Science, 2006;14(2):144-146.

42. Haas CT, Turbanski S, Schmidtbleicher D. Zum Einsatz randomisierter Oszillationen in der Prävention und Rehabilitation von Rupturen des vorderen Kreuzbandes im alpinen Skirennlauf, in Institut für Sportwissenschaften. Frankfurt am Main: J.W. Goethe-Universität Frankfurt am Main; 2002. p. 1-17.

43. Turbanski $S$ et al. Effects of random whole-body vibration on postural control in Parkinson's disease. Res Sports Med. 2005;13(3):243-56.

44. Graziano A, Raulin M. Research Methods: A Process of Inquiry. New York: HarperCollins: 1989.

45. de Bruin ED, van Het Reve E, Murer K. A randomized controlled pilot study assessing the feasibility of combined motor-cognitive training and its effect on gait characteristics in the elderly. Clin Rehabil. 2013;27(3):21525.

46. Cook DJ et al. Prophylaxis of Thromboembolism in Critical Care (PROTECT) Trial: a pilot study. J Crit Care. 2005;20(4):364-72. 



\section{Chapter 6}

Feasibility and informative data after stochastic resonance whole-body vibration intervention in long-term-care dwelling elderly:

a pilot randomised double blinded cross-over study 


\section{ABSTRACT}

\section{Background}

This randomised double-blinded controlled cross-over pilot study examined feasibility and effects of stochastic resonance whole-body vibration training applied in long term care elderly.

\section{Methods}

Nine long term care elderly were recruited and randomized to group A (6 Hz, Noise 4 SR-WBV/ Sham) or B (Sham / $1 \mathrm{~Hz}$, Noise 1 SR-WBV). Feasibility outcomes included recruitment rate, attrition, adherence and safety. Physical performance outcomes focused on the Expanded Timed Get Up-and-Go (ETGUG) test, the Short Physical Performance Battery (SPPB), and lower extremity muscle strength.

\section{Results}

Of 24 subjects initially approached 9 started and 5 completed the study resulting in $37.5 \%$ recruitment, $44.4 \%$ attrition and $81.7 \%$ adherence rates. No adverse events were reported. There is more evidence of improved performance levels in the SR-WBV treatment group with significant differences in average change for isometric rate of force development ( $p=0.016$ left leg; $p=$ 0.028 right leg). No statistical significance was reached for other parameters.

\section{Conclusion}

The findings of this study indicate that the used training protocol for long term care elderly is feasible, however, requires more closely monitoring of participants; e.g. modifications that target improved compliance with the intervention in this setting are required. SR-WBV shows beneficial effects on physical performance for those adhering to the intervention.

Trial registration: U.S. National Institutes of Health NCT01543243 [https://clinicaltrials.gov/ct2/ show/NCT01543243]

\section{Keywords}

feasibility, recruitment, attrition, compliance 


\section{Background}

Physical activity (PA) for elderly is one of the major elements for general health prevention [1] and inactive or sedentary elderly should increase their PA [2]. Despite the known benefits of PA, residents living in long-term care (LTC) are relatively sedentary [3, 4]. Low baseline fitness and mobility levels in (pre-)frail elderly should be considered when starting exercise and this exercise should be adapted to the physical capabilities of these individuals [5].

Whole body vibration (WBV) seems a safe and beneficial type of balance exercise [6, 7]. Pilot studies showed that stochastic resonance WBV (SR-WBV) in (untrained) elderly is both safe and feasible [8, 9]. SR-WBV might also be valuable for (pre-)frail elderly in LTC where the neuromuscular systems of the trainees might not be able withstanding higher loading and long training sessions [8]. However, confirmatory results of such positive effects of WBV in LTC settings is not available and no evidence concerning the feasibility of SR-WBV in LTC dwelling elderly exists.

This study tested the feasibility and effects of SR-WBV training in LTC elderly with the aim to (I) evaluate the intervention process and the ability to recruit and retain LTC elderly for such an intervention, and (II) assess the impact of 4-week SR-WBV on physical performance.

\section{Methods}

\section{Design}

Nine LTC elderly (88.5 \pm 6 years; height: $168 \pm 1 \mathrm{~cm}$; weight: $68.8 \pm 14.3 \mathrm{~kg})$ from "Senevita Residenz Multengut, LTC division Muri, Switzerland" volunteered in this randomised double blind controlled cross-over pilot study. Stratified by sex, participants were randomly assigned to group A or B by means of sealed opaque envelopes distributed after baseline assessments. Following Ethical Committee (Canton Berne) approval, informed consent was obtained prior to training (ClinicalTrial.gov: NCT01543243).

Inclusion criteria were aged over 65 years, being able to stand with or without aids, being classified as Resident Assessment Instrument (RAl [10]) performance level $>0$, scoring $>22$ points on the Mini-Mental Status Examination.

\section{Protocol}

Participants were exposed to SR-WBV using a Zeptor med® device (Frei Swiss AG, Zurich, Switzerland). Participants stood freely on both legs wearing comfortable shoes with slight flexion of the hips, knees and ankle joints. In period 1, participants in group A received 5 sets of 1 minute SR-WBV with $6 \mathrm{~Hz}$, Noise 4 with 1 minute of rest between sets, three times a week, during four weeks. One day rest between training sessions was warranted. Group B received a sham 
intervention of 5 sets of 1 minute SR-WBV with $1 \mathrm{~Hz}$, Noise 1 . The $1 \mathrm{~Hz}$ frequency is expected to cause no training effect [8]. After a wash-out period of 16 days, treatment cross-over took place (Figure1). Secondary outcomes were scheduled at baseline (T0) before training, after four weeks training (T1) in period 1 , and after four weeks training in period 2.

\section{Recruitment rate, rate of loss, program adherence}

The criteria for success were based on the primary feasibility objective focussing on recruitment, attrition and adherence to the intervention [11]. Recruitment of a third of the residents deemed eligible, a 15\% attrition rate, and 70\% training attendance rate were deemed acceptable [12].

\section{Secondary Outcomes}

The reliable Expanded Timed Get Up-and-Go (ETGUG) assessment measured time series of functionally important tasks [13] with details of the protocol reported elsewhere [8]. Physical performance was assessed with the short physical performance battery (SPPB), valid and reliable for lower extremity functions [14] and predictive for disability [15]. A dynamometer tested maximum isometric force (IMVC) and isometric rate of force development (IRFD) of the knee extensors (3 trials of testing were averaged to improve reliability [16]). IRFD was determined from the force-time curve calculating the steepness between the time points of $25 \%$ and $75 \%$ IMVC. Details of the protocol were previously published [16].

\section{Statistical Analysis}

For recruitment, data for the total sampling frame for inclusion in the trial were taken. For the inclusion rate-i.e. the proportion of participants invited to participate who enrolled into the study - we distinguished between those who refused, did not respond or who were willing but excluded. Compliance to the treatment protocol was calculated using "Number of vibration sessions $\div$ the total number of possible vibration sessions $\times 100$ ". Nonparametric Rank-Order Tests of Puri and Sen L Statistics [17] assessed change over time. Effect sizes (ES) were calculated for the differences within and between the groups and expressed as $r=Z / \sqrt{ } N$; where r 0.1 is considered a "small", 0.3 a "medium" and $\geq 0.5$, a 'large' effect [18]. 


\section{Results}

\section{Recruitment, attrition, and adherence}

24 residents were deemed eligible to participate based on the inclusion-exclusion criteria and invited to participate. Nine LTC elderly individuals consented to partake resulting in $37.5 \%$ recruitment rate. Fifteen participants declined with reasons given: no interest $(n=8)$, no motivation $(n=6)$ and personal reasons $(n=1)$. Five participants finished both study periods (age: $85.3 \pm 6$ years; height: $161.4 \pm 6.2 \mathrm{~cm}$; weight: $72.9 \pm 15.9 \mathrm{~kg}$ ) resulting in an attrition rate of $44.4 \%$ ( $n=4$ drop-outs). The reasons for dropping out were: not motivated to continue ( $n=$ $3)$, and heart disease $(n=1)$. Adherence to the intervention for those continuing with training resulted in $81.7 \%$ (98 of 120 training sessions) compliance.

\section{Secondary Outcomes}

Table 1 presents the test results and Table 2 summarises the intervention effects with ES per group for both periods. There are no carry-over effects for any of the outcome measures (Table $1)$, however, within group effect size for the SPPB $(p=0.039)$ is large. Between group effects with large ES are seen for the ETGUG 0 to $2 \mathrm{~m}$, ETGUG 2 to $8 \mathrm{~m}$, IRFD left and IRFD right.

Table 1.

Repeated measures analyses of variance for ranked data for cross-over effects

\begin{tabular}{|c|c|c|c|}
\hline & Pillai's trace $\left(\mathbf{r}^{2}=\mathrm{SS}_{\mathrm{Bet}} / \mathrm{SS}_{\mathrm{Tot}}\right)$ & $L\left[(\mathrm{~N}-1) \mathrm{r}^{2}\right]$ & Probability \\
\hline ETGUG ss & 0.208 & 0.789 & 0.440 \\
\hline ETGUG 0 - $2 \mathrm{~m}$ & 0.536 & 3.462 & 0.160 \\
\hline ETGUG 2 - $8 \mathrm{~m}$ & 0.931 & 4,467 & 0.332 \\
\hline ETGUG turn & 0.833 & 5.000 & 0.167 \\
\hline ETGUG 12 -18 m & 0.833 & 5.000 & 0.167 \\
\hline ETGUG 18 - 20 m & 0.556 & 1,250 & 0.444 \\
\hline ETGUG total time & 0.833 & 5.000 & 0.167 \\
\hline SPPB & 0.932 & 4.559 & 0.329 \\
\hline IMVC, left & 0.556 & 3.750 & 0.148 \\
\hline IMVC, right & 0.556 & 1.250 & 0.444 \\
\hline IRFD, left & 0.772 & 2.600 & 0.278 \\
\hline IRFD, right & 0.893 & 2.778 & 0.409 \\
\hline
\end{tabular}

Abbreviations: ETGUG: Expanded Timed Get Up-and-Go, IMVC: isometric maximal voluntary contraction, IRFD: isometric rate of force development, SPPB: Short Physical Performance Battery, ss: sit to stand movement 


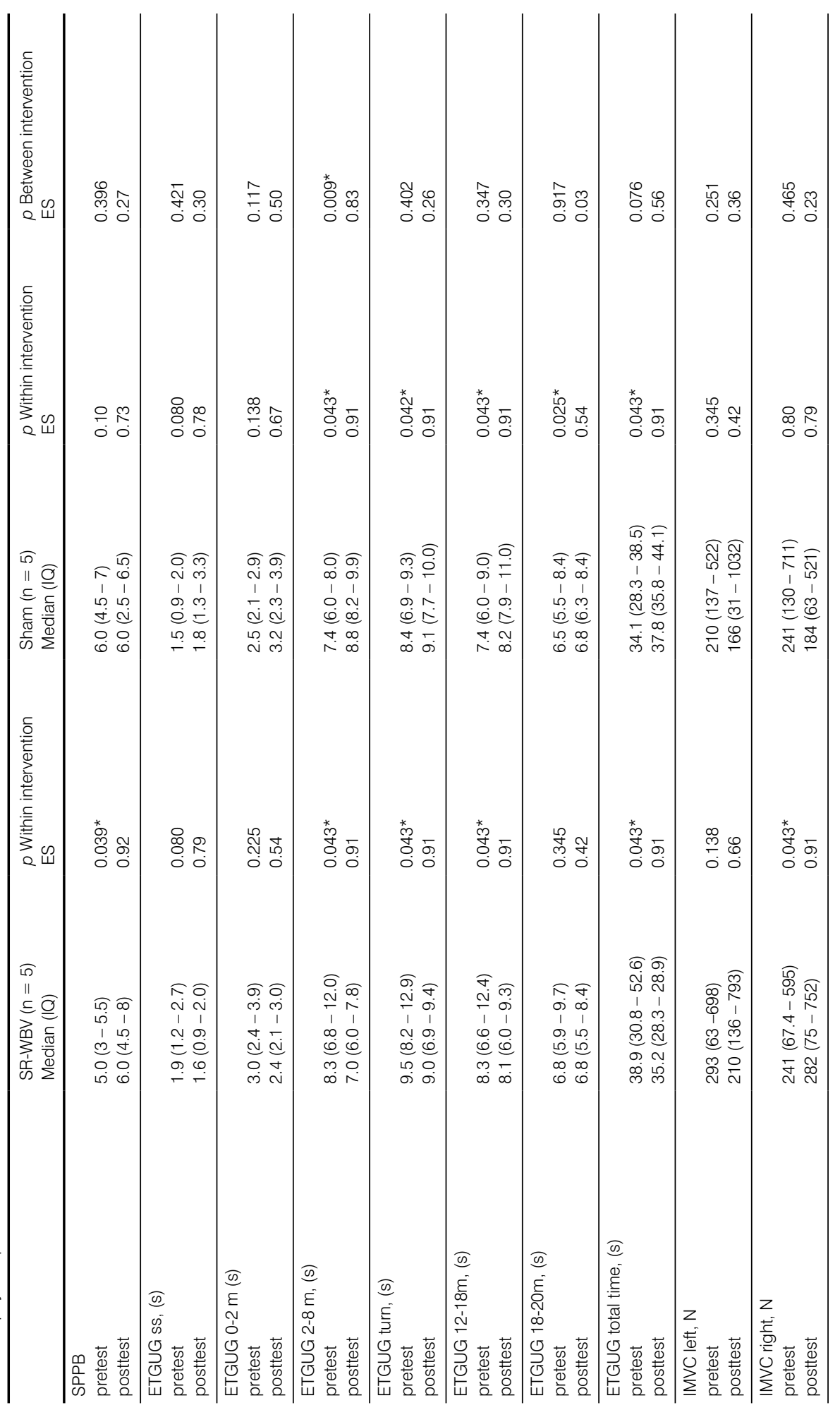




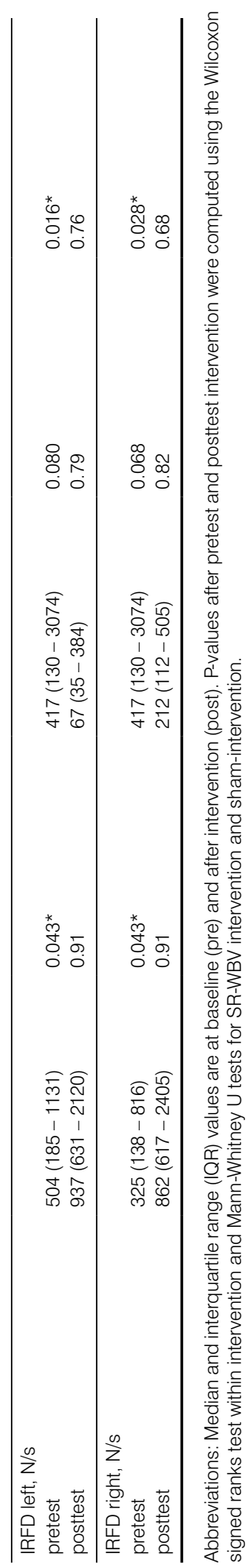




\section{Discussion}

The present pilot study aimed to develop and test a SR-WBV exercise intervention in LTC. The main focus of this study was to evaluate the feasibility of the SR-WBV intervention and the ability to recruit and retain LTC elderly, and to assess the effects of the intervention. Both the aging population and the number of institutionalized older people are expected to increase in the future. Clinical research in long term care is, however, rather still scarce. Research is, therefore, essential to improve the quality of care in LTC homes. Quality of care and improvement thereof in LTC for the aged is, amongst others, relient on future evidence from research projects and their feasibility in real life conditions.

We demonstrated the feasibility of acquiring acceptable recruitment and compliance rates for LTC dwelling older people randomised in this clinical trial. Our targets of 33\% recruitment and $70 \%$ compliance of those training were attained; e.g. those remaining in the study showed excellent compliance with the exercise intervention and retesting. Compared with median rates for recruitment and adherence in falls prevention interventions in institutional settings for clinical trials [12] we achieved better or similar rates for these measures. However, our trial suffered from a rather high attrition rate which we mainly attribute to motivational aspects, e.g. seventeen individuals reported to be not interested or motivated to either begin with exercising or continue to do so. IIIness and personal reasons for withdrawal were only explaining training discontinuation of two individuals. Five of initially nine individuals completed the training programme and retest data were obtained from these individuals. Thus, our trial protocol in its current form is deemed not feasible in LTC because of the high attrition rate. A future study should aim for an attrition rate of around $15 \%$ [12].

From previous studies we know that the presence of a professional exercise instructor working in the facility was significantly associated with exercise participation and with higher exercise frequencies and levels, and session duration [4]. Furthermore, programs must be carefully designed and coached in order to prevent attrition [19] and be focused on the motivation performing functional activities in the LTC setting [20]. Fairhall et al. [21] postulated that frail elderly individuals should be encouraged and supported to adhere to an intervention plan and studies show higher compliance and fewer dropouts for exercise when the program is accompanied by Motivation-Volition (MoVo) programs [22].

Although we are aware of the fact that the emphasis of a pilot study should be placed on feasibility and not on statistical significance [11] our data allow creating a sample size table for various values of the effect or variance estimates to acknowledge the uncertainty surrounding the pilot estimates. For example, based on an estimated meaningful change in SPPB score of 1 point [23], a significance level set at $5 \%$, a power of $80 \%$ to detect differences with two-sided hypothesis testing, inclusion of $\mathrm{N}=30$ participants ( $\mathrm{n}=15$ per group) will be needed for a future two-groups pre- post-test study design. It should be stressed, however, that this sample 
size calculation should be interpreted with caution because our estimates may be unrealistic or biased because of the limited sample size [11].

This pilot study provided useful information about the feasibility of the experimental intervention that used SR-WBV for training. Our subjects tolerated the weekly physical intervention well. Those compliant to training were able to progress in intensity and duration of the exercises. Furthermore, our experience suggests that our SR-WBV was of sufficient duration and/or intensity to ameliorate muscle capacity as indicated by improvements in the secondary outcomes. Pragmatically, however, our experimental subjects did not keep up their motivation to perform regular training three times a week, nor were they willing to do so. We believe, therefore, that it is necessary to proceed to an additional study in LTC, however, with major modifications to the protocol. The modifications should thereby focus on improving the motivation to train in LTC dwelling individuals and also assess benefits or risks [24] of this type of training for older people.

Summarising the findings and limitations of this study it becomes clear that this study only reveals first estimates for the chosen outcome measures. We implemented a strict study design to control threats to validity. A necessary next step would be to adapt the study protocol by adding an intervention component that emphasises motivational aspects of exercising and, thus, strive to improve attrition rates in a new LTC SR-WBV exercise group study design as an additional control procedure.

\section{Conclusions}

We conclude that pilot studies with explicit feasibility objectives and success criteria are important foundation steps in preparing for large trials [11] and for development of rehabilitation research programs. Ongoing formal review of the multifaceted issues inherent in the design and conduct of pilot studies can provide invaluable feasibility and scientific data for rehabilitation specialists working in LTC, e.g. physiotherapists, willing to perform clinical trials [25], and may also be highly relevant for furthering the development of theory based rehabilitation [26]. SR-WBV training as applied in this study is deemed only conditionally feasible; it requires some major modifications to the protocol. However, SR-WBV shows trends to stronger improvement in lower extremity muscle properties when compared with sham training. This study encourages the further development of this intervention, preferably with a randomized control design. Future programs must be carefully designed and coached by professional exercise instructors in order to prevent attrition. 


\section{Abbreviations}

ES: effect size; ETGUG: Expanded Timed Get Up-and-Go; IMVC: isometric voluntary contraction, IRFD: isometric rate of force development, MMSE: Mini-Mental Status Examination, MoVo: Motivation-Volition program PA: physical activity; SR-WBV: stochastic resonance whole-body vibration; WBV: whole-body vibration 


\section{References}

1. Baert V, Gorus E, Mets T, Geerts C, Bautmans I: Motivators and barriers for physical activity in the oldest old: a systematic review. Ageing research reviews 2011, 10(4):464-474.

2. Freiberger E, Sieber C, Pfeifer K: Physical activity, exercise, and sarcopenia - future challenges. Wien Med Wochenschr 2011, 161(17-18):416-425.

3. Benjamin K, Edwards N, Ploeg J, Legault F: Barriers to physical activity and restorative care for residents in long-term care: a review of the literature. Journal of aging and physical activity 2014, 22(1):154-165.

4. De Souto Barreto P, Demougeot L, Vellas $B$, Rolland Y: How much exercise are older adults living in nursing homes doing in daily life? A cross-sectional study. Journal of sports sciences 2015, 33(2):116-124.

5. Gardner MM, Buchner DM, Robertson MC, Campbell AJ: Practical implementation of an exercise-based falls prevention programme. Age and ageing 2001, 30(1):77-83.

6. Chanou K, Gerodimos V, Karatrantou K, Jamurtas $A$ : Whole-body vibration and rehabilitation of chronic diseases: a review of the literature. Journal of sports science \& medicine 2012, 11(2):187-200.

7. Rogan S, Hilfiker R, Herren K, Radlinger L, de Bruin ED: Effects of whole-body vibration on postural control in elderly: a systematic review and meta-analysis. BMC Geriatr 2011, 11:72.

8. Rogan $\mathrm{S}$, Radlinger $\mathrm{L}$, Hilfiker $\mathrm{R}$, Schmidtbleicher D, de Bie RA, de Bruin ED: Feasibility and effects of applying stochastic resonance whole-body vibration on untrained elderly: a randomized crossover pilot study. BMC Geriatrics 2015, 15(25).

9. Rogan $S$, Radlinger L, Schmid S, Herren K, Hilfiker R, de Bruin ED: Skilling up for training: a feasibility study investigating acute effects of stochastic resonance whole-body vibration on postural control of older adults. Ageing Research 2012, 3(1:e5).

10. Carpenter I, Hirdes JP: Using interRAI assessment systems to measure and maintain quality of long-term care. In: A Good Life in Old Age? Monitoring and Improving Quality in Long-term Care. OECD Publishing; 2013: 263.

11. Thabane L, Ma J, Chu R, Cheng J, Ismaila A, Rios LP, Robson R, Thabane M, Giangregorio L, Goldsmith $\mathrm{CH}$ : A tutorial on pilot studies: the what, why and how. BMC medical research methodology 2010, 10:1.

12. Nyman SR, Victor CR: Older people's recruitment, sustained participation, and adherence to falls prevention interventions in institutional settings: a supplement to the Cochrane systematic review. Age and ageing 2011, 40(4):430-436.

13. Botolfsen P, Helbostad JL, Moe-Nilssen R, Wall JC: Reliability and concurrent validity of the Expanded Timed Up-and-Go test in older people with impaired mobility. Physiotherapy research international : the journal for researchers and clinicians in physical therapy 2008, 13(2):94-106.

14. Guralnik JM, Simonsick EM, Ferrucci L, Glynn RJ, Berkman LF, Blazer DG, Scherr PA, Wallace RB: A Short Physical Performance Battery Assessing Lower- 
Extremity Function - Association with Self-Reported Disability and Prediction of Mortality and Nursing-Home Admission. J Gerontol 1994, 49(2):M85-M94.

15. Guralnik JM, Ferrucci L, Simonsick EM, Salive ME, Wallace RB: Lower-Extremity Function in Persons over the Age of 70 Years as a Predictor of Subsequent Disability. New Engl J Med 1995, 332(9):556-561.

16. Mebes C, Amstutz A, Luder G, Ziswiler $\mathrm{HR}$, Stettler M, Villiger PM, Radlinger $\mathrm{L}$ : Isometric rate of force development, maximum voluntary contraction, and balance in women with and without joint hypermobility. Arthritis and rheumatism 2008, 59(11):1665-1669.

17. Thomas JR, Nelson JK, Thomas KT: A generalized rank-order method for nonparametric analysis of data from exercise science: a tutorial. Res Q Exerc Sport 1999, 70(1):11-23.

18. Corder GW, Foreman DI: Nonparameztric statistics for non-statisticans . a step-bystep approach. Hoboken, New Jersey: John Wiley \& Sons. Inc.; 2009.

19. Zech A, Drey M, Freiberger E, Hentschke C, Bauer JM, Sieber CC, Pfeifer K: Residual effects of muscle strength and muscle power training and detraining on physical function in community-dwelling prefrail older adults: a randomized controlled trial. BMC Geriatr 2012, 12:68.

20. Resnick B: Functional performance of older adults in a long-term care setting. Clinical nursing research 1998, 7(3):230-246; discussion 246-239.

21. Fairhall N, Langron C, Sherrington C, Lord SR, Kurrle SE, Lockwood K, Monaghan N, Aggar C, Gill L, Cameron ID: Treating frailty-a practical guide. BMC medicine 2011,
9:83.

22. Fuchs R, Goehner W, Seelig H: Long-term effects of a psychological group intervention on physical exercise and health: the MoVo concept. Journal of physical activity \& health 2011, 8(6):794-803.

23. Kwon S, Perera S, Pahor M, Katula JA, King AC, Groessl EJ, Studenski SA: What is a meaningful change in physical performance? Findings from a clinical trial in older adults (the LIFE-P study). J Nutr Health Aging 2009, 13(6):538-544.

24. Brooke-Wavell K, Mansfield NJ: Risks and benefits of whole body vibration training in older people. Age and ageing 2009, 38(3):254-255

25. Cook DJ, Rocker G, Meade M, Guyatt G, Geerts W, Anderson D, Skrobik Y, Hebert P, Albert M, Cooper J et al: Prophylaxis of Thromboembolism in Critical Care (PROTECT) Trial: a pilot study. Journal of critical care 2005, 20(4):364-372.

26. de Bruin ED, van Het Reve E, Murer K: A randomized controlled pilot study assessing the feasibility of combined motor-cognitive training and its effect on gait characteristics in the elderly. Clinical rehabilitation 2013, 27(3):215-225. 


\section{Additional material}

\section{Additional file 1.}

Description of the demographic variables of the participants included in the analysis

\begin{tabular}{lllllll}
\hline Participant & Age & Gender & Height $\mathbf{( c m})$ & Weight $\mathbf{( k g )}$ & BMI $\mathbf{( k g / \mathbf { m } ^ { 2 } )}$ & Walking aids \\
\hline 1 & 89 & male & 169 & 78.7 & 27.6 & walking frame \\
2 & 88 & female & 164 & 59.0 & 21.9 & walking frame \\
3 & 82 & female & 152 & 73.4 & 31.8 & walking frame \\
4 & 91 & female & 162 & 57.2 & 21.8 & walking frame \\
5 & 77 & male & 160 & 96.1 & 37.5 & walking frame \\
\hline
\end{tabular}





\title{
Chapter 7
}

\author{
Sensory-motor training targeting motor \\ dysfunction and muscle in long-term-care elderly \\ combined with motivational strategies: \\ a single blind randomized controlled study
}




\section{ABSTRACT}

\section{Background}

This study evaluated the effects of a combined innovative training regime consisting of stochastic resonance whole-body vibration (SR-WBV) and a dance video game (DVG) on physical performance and muscle strength in long-term-care dwelling elderly.

\section{Methods}

Thirthy long-term-care elderly were randomly allocated to an intervention group (IG; $\mathrm{n}=16$ ) receiving combined SR-WBV training and DVG, or a sham group (SG; $n=14$ ). IG performed five sets one minute of SR-WBV, with one minute rest between sets (base frequency $3 \mathrm{~Hz}$ up to $6 \mathrm{~Hz}$, Noise 4) during the first five weeks on three days per week. From week five to eight a DVG was added to SR-WBV for IG on three days per week. SG performed a five-set SR-WBV program (1 $\mathrm{Hz}$, Noise 1) lasting five times one minute, with one minute rest in between, three days a week. From week five to eight stepping exercises on a trampoline were added on three days per week. Primary outcome: Short physical performance battery (SPPB). Secondary outcome: isometric maximal voluntary contraction (IMVC), and sub phases of IMVC (Fsub), isometric rate of force development (IRFD) and sub time phases of IRFD (IRFDsub) were measured at baseline, after four and eight weeks. ANOVA with repeated measures was used for analyses of time and interaction effects and MANOVA determined between group intervention effects.

\section{Results}

Between group effects revealed significant effects on the SPPB primary outcome after four weeks $F(1,27)=6.17 ; p=0.02)$ and after eight weeks $F(1,27)=11.8 ; p=0.002)$. Secondary muscle function related outcome showed significant between group effects in IG on IRFD, Fsub $30 \mathrm{~ms}, 100 \mathrm{~ms}$, 200ms and IRFDsub 0-30ms, 0-50ms, 0-100ms and 100-200ms compared to SG (all $p<0.05)$.

\section{Conclusions}

Eight weeks SR-WBV and DVG intervention improved lower extremity physical function and muscle strength compared to a sham intervention in long-term-care elderly. SR-WBV and DVG seems to be effective as a training regime for skilling up in long-term-care elderly.

Keywords: Sensory-motor training, motor dysfunction, muscle weakness, long-term care, motivational strategies 


\section{Background}

'The competence of an individual to have the physiological capacity to perform normal everyday activities safely and independently without undue fatigue' [1] signifies the functional abilities of an individual. Disability, defined as difficulty or dependency in the execution of the activities of daily living, is associated with increased healthcare utilization and related costs [2]. Disability in frail older people is considered a public health problem [3] in which prevention has to be considered a priority for research and clinical practice [4]. Physical activity (PA) for the elderly is one of the major elements for general health prevention [5]; therefore inactive or sedentary elderly should increase their PA [6]. Despite the known benefits of PA, residents living in longterm care (LTC) are relatively sedentary $[7,8]$.

The loss of muscle mass and strength with age, coined sarcopenia, is recognized as a major cause of disability and morbidity in the elderly [9]. Sarcopenia describes the progressive decline in skeletal muscle mass and function (strength or performance) with advancing age [10]. However, recent studies demonstrated that muscle atrophy is a relatively small contributor to the loss of muscle strength [11-13]. Changes in neurologic function and/or the intrinsic force-generating properties of skeletal muscle are recently proposed to be responsible for muscle weakness and motor dysfunction in the elderly [11, 14-18]. Dynapenia has been used to coin this age-associated loss of muscle strength and power with its significant clinical consequences; e.g., the increased risk for functional limitations, disability, and mortality. Dynapenia encompasses broader aspects of skeletal muscle performance, and so includes strength (i.e., maximal voluntary force) and/or mechanical power (a product of force - time velocity) [19] together with aspects of neurological functioning $[20,21]$.

Both neurologic and skeletal muscle properties are necessary for optimal muscle force production and control $[16,17,22]$. The nervous system's ability to fully activate a skeletal muscle voluntarily for example seems to be impaired in individuals with dynapenia [23]. Furthermore, poor sensorimotor nerve function independently predicts mobility disability [24]. Targeting neural structures through exercise is, therefore, considered important in influencing muscle strength in elderly [25].

Efficient movement function and the maintenance of balance function during dynamic tasks; e.g., during walking, are more complex than merely adequate force production from the muscles [26]. For whole body movements it is important to precisely coordinate muscle actions. This requires sensory, biomechanical and motor-processing strategies along with learned responses from previous experiences and anticipation of change [27, 28]. Adequately combining three levels of motor control (spinal reflex, brain stem balance, and cognitive programming) produces appropriate muscle responses [29]. So, from these results, it can be hypothesised that when focusing on these three levels of motor control in a training program there will be improvements 
in muscle recruitment and timing and, hence, physical functioning and muscle strength.

Stochastic resonance (SR) is a phenomenon in nonlinear systems characterized by a response increase of the system induced by a particular level of input noise [30, 31]. One of the first studies applying noise in humans revealed increased sensitivity to detect sub-threshold tactile stimuli as an effect to such an intervention [32]. Cordo and colleagues (1996) were among the first to demonstrate that the application of noise on human muscle spindle receptors improved afferents sensitivity in the human motor system and suggested, based on their results, that a stochastic-resonance based technique could be applied in clinical settings to individuals with elevated cutaneous thresholds; e.g. to older adults [33]. First evidence that mechanical noise applied to the feet via vibrating insoles improved balance in standing position stems from Pripatla et al. (2002) [34] and Collins and co-workers (2003) [35]. Systematic reviews concluded that, compared to more demanding interventions, whole-body vibration (WBV) as a sensorimotor training might be a safer and less fatiguing type of exercise [36] with a beneficial effect on movement skills [37] and muscle strength [38]. Stochastic resonance whole-body vibration (SR-WBV) has been described as stimulating sensorimotor processes [31, 39] with a positive effect on muscle functional strength [38]. The SR-WBV stimulus triggers muscle spindles and, thereby, improves the functionality of the muscle-nerve system [40] and adjusts afferent and efferent signals which, in turn, are leading to "training" effects for the sensorimotor system [41]. Muscle strength increase following SR-WBV is mainly attributed to neural adaptation bringing on improvements in inter- and intra-muscular coordination [42]. See [43] for an overview. Virtual reality training techniques may be used to incorporate cognitive programming elements into exercise [44] and could, hence, also be part of a training program for elderly [45]. Pilot trials with long term care dwelling elderly showed beneficial effects on physical performance for those adhering to an SR-WBV intervention, however, the program requires modifications that target improved compliance with the intervention [46]. Interventions performed with frail individuals often suffer from low adherence rates and are, therefore, advised to specifically include support and motivation strategies, as well as giving assistance to individuals to develop both goals and the strategies to achieve these $[47,48]$.

The aim of this study was to assess the effects of a sensorimotor training program with SRWBV \& Virtual Reality Training that was accompanied with motivational instructions in LTC elderly on lower extremity physical function and leg muscle properties. We hypothesised that an intervention program that targets motor control will effect on physical functioning and muscle strength of LTC elderly. 


\section{Methods}

\section{Design}

This study follows the publication guideline of CONSORT [49], in the form of a randomised controlled trial with blinded LTC dwelling elderly individuals, randomly divided over intervention (IG) and sham control (SG) groups. The assessor and supervisors were not blinded. Measurements were carried out at baseline (BASE), after four weeks (4W) and after eight weeks (8W) of training. Data were collected and analysed for participants that completed $90 \%$ of the scheduled training sessions. Figure 1 presents the study flow.

\section{Participants}

Inclusion criteria was: age over 65 years, able to stand with or without walking aids, living in the Canton of Berne, being classified as Resident Assessment Instrument (RAI) ${ }^{3}$ performance level $>0$, having a score $\geq 18$ in the Mini-Mental Status Examination (MMSE) Test and $\leq 6$ points on the Short Physical Performance Battery. Scoring $\leq 6$ points on the Short Physical Performance Battery relates to poor physical performance and is a risk indicator for sarcopenia [50]. Exclusion criteria were: visual disturbances ${ }^{b}$, lower or upper leg prosthesis, acute joint disease, acute thrombosis, acute fractures, acute infections, acute tissue damage, acute surgical scars or alcohol abuse. The sampling frame of LTC elderly fulfilling the inclusion/exclusion criteria and receiving information about the study comprised 40 individuals (Table 1). Thirty-one LTC elderly agreed to participate and were reached through personal interview and/or public information events.

\section{Table 1.}

Demographic characteristics at baseline (mean \pm SD)

\begin{tabular}{lccc}
\hline & IG & SG & p \\
\hline Sample & 16 & 14 & \\
Sex (F/M) & $10 / 6$ & $10 / 4$ & 0.156 \\
Age (years) & $90.4 \pm 6.9$ & $87.2 \pm 5.0$ & \\
Age (range) & $77-100$ & $79-97$ & 0.256 \\
Height (m) & $1.63 \pm 0.1$ & $1.58 \pm 0.1$ & 0.969 \\
Weight $(\mathrm{kg})$ & $66.9 \pm 14.2$ & $67.1 \pm 20.8$ & 0.542 \\
BMl $\left(\mathrm{kg} / \mathrm{cm}^{2}\right)$ & $25.1 \pm 4.8$ & $26.5 \pm 6.8$ & \\
\hline
\end{tabular}

Abbreviation: IG: intervention group, SG: sham group, F: female, M: male, BMI: Body mass index, SPPB: Short Physical Performance Battery

3 The ADL Hierarchy Scale of the RAI groups activities of daily living according to the stage of the disablement process in which they occur. Early loss ADLs (for example, dressing) are assigned lower scores than late loss ADLs (for example, eating). The ADL Hierarchy ranges from 0 (no impairment) to 6 (total dependence); source: Morris JN, Fries BE, Morris SA. (1999) Scaling ADLs within the MDS. Journals of Gerontology: Medical Sciences 54(11):M546-M553) 


\section{Randomisation}

An independent research assistant performed randomisation, using a random Microsoft Excel 2010 table. The participants were randomly assigned to either IG or SG by means of sealed opaque envelopes distributed after the completion of all baseline assessments. Prior to the start of the intervention, written informed consent was obtained from all participants following Ethical Committee approval (Canton Berne; Registration number 147/12). The study protocol was registered at U.S. National Institute of Health (NCT02102919; https://clinicaltrials.gov/ct2/ show/NCT02102919).

\section{Intervention Protocol}

All participants were familiarised with the SR-WBV and the Dance Video Game (DVG) device one week prior to the intervention period with the aim to reduce anxiety. In the DVG session a tutorial sequence was provided to ensure understanding of the task.

\section{Stochastic resonance whole-body vibration}

Participants were exposed to SR-WBV using a Zeptor med ${ }^{\circledR}$ device (Frei Swiss AG, Zurich, Switzerland) while standing freely on both legs wearing no shoes with slight flexion of the hips, knees and ankle joints. A purpose made seat was used to allow participants to sit down during the one-minute breaks. Each intervention consisted of five one-minute vibration periods with a one-minute break between sets. The intervention took place on three days a week, over a period of eight weeks. IG vibrated with a basic frequency of $3 \mathrm{~Hz}$ and noise level 4 since a previous study showed this to be a good starting level for our target population [51]. To ensure progression of the exercise, the basic frequency gradually increased to $6 \mathrm{~Hz}$, depending on capabilities and feedback of the individual concerned. The amplitude was a constant $4 \mathrm{~mm}$ during the 8 weeks period. In analogy to previously described protocols [52] progression of the exercise intensity was based on participant's abilities related to maintain postural stability with decreasing base of support: from being able to stand with parallel positioned feet without holding onto the bars, the vibration frequency was increased gradually from $3 \mathrm{~Hz}$ up to $6 \mathrm{~Hz}$. From $6 \mathrm{~Hz}$, the parallel standing position changed to tandem standing and ended with this position while dynamic squat movements were performed on the vibrating plates. SG vibrated with a basic frequency of $1 \mathrm{~Hz}$ and noise level 1 with no increase of the basic frequency and no additional exercises. The $1 \mathrm{~Hz}$ frequency was expected to cause no training effect [53] as previously shown [54-56].

\section{Dance video game}

As an additional cognitive element the DG performed the dance video game after the SR-WBV exercise in every training session. The dance video game was performed on metal dance pads (TX 6000 Metal DDR Platinum Pro, 93 x 14.7 x 109 cm, Mayflash Limited, Baoan Shenzhen, China) and with a specially designed modification of the StepMania (Version 3.9) free-ware [44, $57,58]$. The dance video game screen was projected on a white wall. A scrolling display of arrows moving upwards across the screen cued each move, and the participants were asked 
to execute the indicated steps (forward, backward, right, or left) when the arrows reached the fixed raster graphic at the top of the screen, and in time with different songs (32 to 137 beats per minute). For each training session, the participants performed for four songs of two to three minutes each, with a short break of 30 seconds after each song. Progression of performance was controlled through beats per minute and difficulty level and adapted based on performance of an individual.

As the levels increased, additional distracting visual cues, e.g., "bombs," were presented. Participants had to ignore these cues and keep their attention focused on the arrows. Occasionally, some arrows were drawn-out on the target locations indicating that the participants should remain for a while on the dance pad button on one leg. The arrow sequences were generated using the Dancing Monkey MATLAB script [59] and determined step error. Electronic sensors in the dance pad detected position and timing information that was then used to provide participants with real-time visual feedback.

Video game dancing (DANCE) promotes fast, rhythmic, and accurate foot movements and may improve higher cognitive processing as measured by standard neuropsychological tests [60, $61]$.

\section{Motivation stategy}

A professional exercise instructor (SR) coached the participants in both groups to enhance exercise participation and ensure that the targeted exercise frequencies and levels, sessions duration [8], and prevention of attrition [62] would be reached. The exercise instructor focused on the motivation to perform functional activities in the LTC setting [63] with the help of MotivationVolition (MoVo) [64]. MoVo programs, where the acronym stands for "motivation" and "volition", have previously shown to be succesfull in reaching higher compliance and fewer dropout rates for exercise in in-patient rehabilitation settings [65]. In brief, MoVo intervention programs encompass the following motivational strategies: (a) clarification of personal health objectives; (b) contemplation of different actions to achieve the health objectives; (c) formation of specific goal intentions; (d) checking self-concordance of this goal intention; and ( $f$ ) reflection of outcome experiences. Furthermore, MoVo puts a strong emphasis on subsequent volitional strategies: (a) generating implementation intentions; (b) anticipating personal barriers; (c) developing counter strategies; and (d) self-monitoring the new behavior. The specific features of MoVo are described in more detail elsewhere [64].

\section{Primary outcome}

The Short Physical Performance Battery (SPPB) [66], a standardized measure of physical performance that assesses standing balance, usual gait velocity over a 4-m course, and the time to sit down and rise from a chair five times as quickly as possible, has been recommended for use as a functional outcome measure in clinical trials in frail older persons [67]. The SPPB 
shows high reliability with an ICC of 0.88-0.92 [68]. SPPB scores range from 0 (lowest function) to 12 (highest function) points and can be classified as: "weak performance" for scores from 0 to 6 points, "mean performance" for 7 to 10 points, "good performance" for 11 to12 points [69]. The SPPB scale predicts institutionalization, hospital admission, mortality, and disability [66, 70, 71].

\section{Secondary outcomes}

Isometric maximal voluntary contraction (IMVC) and isometric rate of force development (IRFD) of knee extension and knee flexion were measured. The onset of force was determined at 10 Newton ( $\mathrm{N}$ ) of each individual's force-time curve. IMVC in $\mathrm{N}$ was determined as the maximum point on the force-time curve. Submaximal force (Fsub) values were calculated at 30, 50, 100 and $200 \mathrm{~ms}$ relative to the onset of force and presented the maximum voluntary contraction values $(N)$ at this time point. IRFD was defined as the maximal slope of the force-time curve between onset of force and 200ms ( $\mathrm{N} / \mathrm{ms}$ ). Submaximal IRFD values (IRFDsub) were calculated as the mean slope of the force-time curve $(\Delta \mathrm{F} / \Delta \mathrm{t})$ over time intervals of $0-30,0-50,0-100$ and $100-200 \mathrm{~ms}$ relative to the onset of force [72]. The onset of force was $0 \mathrm{~ms}$.

A strain gauge (Sensor KM 1500S, Megatron, Munich, Germany) was used to measure the force values. The participants sat on a chair with $90^{\circ}$ knee flexion and the dynamometers were fixed above the right ( $r$ ) and left (I) ankle joint (Figure 2). On the command "3-2-1-go!" the participants had to flex (flex) or extend (ex) each knee separately for five seconds as fast and as strongly as possible against the fixation. Details of the protocol were previously published and result in reliable measures with intraclass correlation coefficients (ICC) ranging between from 0.90 to 0.98 [73]. The analogue signal of the dynamometer was transmitted to a measurement amplifier (UMV, uk-labs, Kempen, Germany), digitalised by a 12-bit A-/D-converter (Meilhaus ME-2600i, SisNova Engineering, Zug, Switzerland) with a sampling rate of $1 \mathrm{kHz}$ and analyzed with the Analogue Digital Signal processing software (ADS, uk-labs, Kempen, Germany).

\section{Data Processing}

Dynamometer data was processed with a costum-made software routine (MATLAB R2013a). After low-pass filtering ( $10 \mathrm{~Hz}, 2^{\text {nd }}$ order Butterworth, forward-backward) the force-time curves were visually inspected for plausibility and to determine the time points of force onset $(10 \mathrm{~N})$. Force parameters were then calculated according [72] the respective time windows.

\section{Sample size calculation}

The primary study objective was to evaluate the effect of SR-WBV and DVG compared to sham intervention on lower extremity physical performance as assessed with the SPPB. Based on an estimated meaningful change in SPPB score of 1 point [74-76], a significance level set at 5\%, a power of $80 \%$ to detect differences with two-sided hypothesis testing, inclusion of $\mathrm{N}=30$ participants ( $n=15$ per group) will be needed for a two-groups pre- post-test study design. 


\section{Statistical analyses}

Statistical Package for Social Sciences (SPSS) 22.0 for Mac (SPSS, Inc; Chicago, Illinois) was used for all statistical analyses. An intention-to-treat analysis (ITT) was performed where missing data were replaced by mean values of the group to which subjects were originally allocated [77]. Kolmogorov-Smirnov test was used to assess normality of data distribution. Baseline demographic data and baseline outcome data comparing IG and SG were analyzed by unpaired students t-test.

A 2 (groups) x 3 (measurements) repeated measures analysis of variance (ANOVA) was carried out to test for interactions and time effects (Greenhouse-Geisser corrected). We used nonparametric Rank-Order Tests of Puri and Sen L Statistics to assess change over time where Pillai's Trace was used to calculate L [78-80].

For effect sizes (ES) assessing meaningfulness of differences within and between groups, etasquared $\left(\eta^{2}\right)$ in ANOVA and MANOVA was used. For $\eta^{2}$, an effect size of 0.01 is considered a 'small' effect, around 0.06 a 'moderate' effect and 0.14 and above a 'large' effect [81].

\section{Results}

From the 40 LTC elderly approached, 31 agreed to participate (Figure 1) resulting in a $77.5 \%$ recruitment rate for the sampling frame. One participant from SG died before baseline measurement and eight of the 40 initially deemed eligible were willing to participate, however, did not fulfill the inclusion criteria ( $n=3$ low MMSE score; $n=4$ with recent stroke; $n=1$ with multiple sclerosis, $n=1$ with parkinson disease). Training adherence rate, expressed in \%; [100 $\div$ (34 $\div$ Mean amount of trainings visited)] revealed a mean attendance rate of $100 \%$ (34 of 34 intervention sessions).

The participants were willing to be randomized. Neither subjective nor objective side-effects related to the used intervention were reported. At baseline, no statistically significant differences $(p<0.05)$ were found between groups.

All SPPB data could be used for statistical analysis while for strength values an ITT was performed. The time force-curve of four participants could not be used for analysis. Fsub values and IRFDsub are listed in additional files 1 - 16. At baseline no group differences were identified.

\section{Primary outcome: Short Physical Performance Battery}

A significant interaction effect in SPPB; $F(1.7,48)=35.2, p<0.001)$ with a large $E S(n 2=0.557)$ were determined in favour of IG (Table 2). The between group effect (Table 5) shows significant values after 4 weeks $F(1,28=6.85 ; \eta 2=0.20 ; p=0.014)$ and after 8 weeks $F(1,28=13.17 ; \eta 2$ $=0.32 ; p=0.001$ ) in favour of IG. 
Table 2.

Overview for ANOVA with repeated measures of SPPB outcome data

\begin{tabular}{lllll}
\hline & $\begin{array}{l}\text { Pillai } \mathbf{s} \text { trace } \\
\left(\mathbf{r}^{2}=\mathbf{S S}_{\left.\text {Bet } / \mathbf{S S}_{\text {Tot }}\right)}\right.\end{array}$ & $\mathbf{L}\left[(\mathbf{N}-\mathbf{1}) \mathbf{r}^{2}\right]$ & $\mathbf{p}$ & ES (n2) \\
\hline SPPB Total (time effects) & 0.22 & 0.30 & 0.742 & 0.22 \\
SPPB Total (interaction effects) & 0.60 & 10.20 & $0.001^{*}$ & 0.60 \\
\hline
\end{tabular}

Abbreviation: SPPB: Short Physical Performance Battery; ${ }^{\circ}$ : significant difference $p<0.05$, *: significant difference after Bonferroni adjustment $\mathrm{p}<0.0125$; ES: effect size ( $\mathrm{\eta} 2=.01$; small effect, $\eta 2=.06$; moderate effect, $\eta 2=.14$; large effect)

\section{Secondary outcomes: strength tests}

Tables 3-5 summarise the intervention effects for the muscle strength related outcomes. IMVC showed significant changes over time for knee extension right and left and knee flexion right (Table 4). Post-hoc analysis revealed significant between group effects for knee flexion left ( $p<$ 0.02) after eight weeks of training (Table 5).

Following eight weeks of SR-WBV and DVG training, Fsub measures at 30ms, 100ms and 200ms in both right and left leg flexion showed a significant between group effect $(p<0.01)$ with large ES (>0.14) compared to Sham intervention (additional files Figures 1 - 8).

Table 3 shows the Greenhouse-Geisser univariate test results; a significant intragroup-by-time effect and group-by-time interaction effect following eight weeks of SR-WBV and DVG intervention on IRFD. Significant between groups effects were both seen after 4 weeks $(p<0.05)$ and 8 weeks $(p<0.05)$ in IRFD right and left leg extension and right and left leg flexion manoeuvres (Table 5).

\section{Table 3}

ANOVA with repeated measurements (ranks) intergroup-by-time effects and group-by-time interaction for the secondary outcomes IMVC (N).

\begin{tabular}{|c|c|c|c|c|}
\hline & $\begin{array}{c}\text { Pillai's trace }\left(r^{2}=\right. \\
\left.\mathrm{Ss}_{\mathrm{Bet}} / \mathrm{sS}_{\mathrm{TW}}\right)\end{array}$ & $L\left[(N-1) r^{2}\right]$ & p & ES $\left(n^{2}\right)$ \\
\hline IMVC right ex (N) (time effects) & 0.001 & 0.006 & 0.994 & 0.001 \\
\hline IMVC right ex (N) (interaction effects) & 0.28 & 5.41 & $0.01^{*}$ & 0.28 \\
\hline IMVC left ex (N) (time effects) & 0.01 & 0.007 & 0.993 & 0.001 \\
\hline IMVC left ex (N) (interaction effects) & 0.34 & 7.16 & $0.003^{*}$ & 0.51 \\
\hline & 0.27 & 1.62 & 0.232 & 0.27 \\
\hline IMVC right flex (N) (time effects) & 0.001 & 0.003 & 0.997 & 0.001 \\
\hline IMVC night flex (N) (interaction effects) & 0.15 & 2.54 & 0.097 & 0.15 \\
\hline IMVC left fex (N) (time effects) & 0.001 & 0.001 & 0.999 & 0.001 \\
\hline IMVC left flex (N) (interaction effects) & 0.09 & 1.33 & 0.282 & 0.09 \\
\hline
\end{tabular}

Abbreviation: IMVC: isometric maximum voluntary contraction; ${ }^{\circ}$ : significant difference $p<0.05$, * significant difference after Bonferroni adjustment $\mathrm{p}<0.0125$; ES: effect size $(\eta 2=.01$; small effect, $\eta 2=.06$; moderate effect, $\eta 2=.14$; large effect) 


\section{Table 4}

ANOVA with repeated measurements (ranks) intergroup-by-time effects and group-by-time interaction for the secondary outcomes IRFD (N/ms)

\begin{tabular}{|c|c|c|c|c|}
\hline & Pillai s trace $\left(\mathrm{r}^{2}=\mathrm{SS}_{\mathrm{Bet}} / \mathrm{SS}_{\mathrm{T} \phi)}\right)$ & $\mathbf{L}\left[(\mathrm{N}-1) \mathrm{r}^{2}\right]$ & $\mathbf{p}$ & $\operatorname{ES}\left(\eta^{2}\right)$ \\
\hline IRFD right ex (N/ms) (time effects) & 0.001 & 0.005 & 0.995 & 0.001 \\
\hline IRFD right ex (N/ms) (intenction effects) & 0.238 & 4.37 & $0.022^{*}$ & 0.24 \\
\hline IRFD left ex (N/ms) (time effects) & 0.43 & 10.73 & $0.001^{*}$ & 0.43 \\
\hline IRID left ex (N/ms) (interaction effects) & 0.02 & 0.03 & 0.97 & 0.002 \\
\hline IRFD right flex (N/ms) (time effects) & 0.68 & 29.38 & $0.001^{*}$ & 0.68 \\
\hline IRFD right flex (N/ms) (interaction effects) & 1.45 & 6.30 & $0.007^{*}$ & 0.59 \\
\hline IRFD left flex (N/ms) (time effects) & 0.85 & 52.61 & $0.001^{*}$ & 0.85 \\
\hline IRFD left flex (N/ms) (intenaction effects) & 0.52 & 9.65 & $0.001^{*}$ & 0.52 \\
\hline
\end{tabular}

Abbreviation: IRFD: isometric rate of force development; ': significant difference $p<0.05$, *: significant difference after Bonferroni adjustment $\mathrm{p}<0.0125$; ES: effect size ( $\eta 2=.01$; small effect, $\eta 2=.06$; moderate effect, $\eta 2=.14$; large effect)

Significant effects $(p<0.001)$ and large ES $>0.14$ in the right and left leg knee extension and knee flexion movements were shown for IRFDsub at 0-30ms, 0-50ms, 0-100ms and 100-200ms (additional file Figure 9 - 16).

\section{Table 5.}

Between group effects at BASE, 4W and 8W on SPPB, IMVC and IRFD

\begin{tabular}{|c|c|c|c|c|c|c|}
\hline & BASE & $\mathrm{p} / \mathrm{n} 2$ & $4 W$ & $p / n 2$ & $8 W$ & $\mathrm{p} / \mathrm{n} 2$ \\
\hline SPPB (Score) (IG) & $2.9 \pm 1.7$ & $0.16 / 0.07$ & $5.6 \pm 2.9$ & $0.01 * / 0.21$ & $7.13 \pm 3.2$ & $0.004 * / 0.26$ \\
\hline SPPB (Score) (SG) & $3.9 \pm 1.5$ & & $3.4 \pm 1.2$ & & $3.7 \pm 1.2$ & \\
\hline IMVC right ex (N) (IG) & $136.0 \pm 58.4$ & $0.62 / 0.01$ & $138.4 \pm 56.0$ & $0.81 / 0.002$ & $180.1 \pm 71.2$ & $0.10 / 0.09$ \\
\hline IMVC right ex (N) (SG) & $157.9 \pm 75.7$ & & $134.4 \pm 59.0$ & & $147.8 \pm 62.8$ & \\
\hline IMVC left ex (N) (IG) & $140.4 \pm 89.3$ & $0.97 / 0.001$ & $163.1 \pm 78.1$ & $0.12 / 0.08$ & $194.6 \pm 90.2$ & $0.04^{\circ} / 0.26$ \\
\hline IMVC left ex (N) (SG) & $132.5 \pm 56.0$ & & $119.5 \pm 54.0$ & & $126.1 \pm 65.7$ & \\
\hline IMVC right flex (N) (IG) & $61.0 \pm 29.0$ & $0.88 / 0.001$ & $74.4 \pm 30.7$ & $0.29 / 0.04$ & $87.0 \pm 38.5$ & $0.08 / 0.11$ \\
\hline IMVC right flex (N) (SG) & $64.1 \pm 31.0$ & & $67.0 \pm 31.7$ & & $67.0 \pm 31.2$ & \\
\hline IMVC left flex (N) (IG) & $73.5 \pm 42.6$ & $0.37 / 0.03$ & $79.0 \pm 44.8$ & $0.15 / 0.07$ & $86.9 \pm 36.8$ & $0.03^{\circ} / 0.15$ \\
\hline IMVC left flex (N) (SG) & $60.3 \pm 24.5$ & & $60.4 \pm 25.3$ & & $64.4 \pm 16.5$ & \\
\hline IRFD right ex (N/ms) (IG) & $0.46 \pm 0.3$ & $0.70 / 0.005$ & $0.56 \pm 0.3$ & $0.02 * / 0.06$ & $0.72 \pm 0.4$ & $0.004 * / 0.25$ \\
\hline IRFD right ex (N/ms) (SG) & $0.41 \pm 0.3$ & & $0.40 \pm 0.2$ & & $0.39 \pm 0.1$ & \\
\hline IRFD left ex (N/ms) (IG) & $0.48 \pm 0.4$ & $0.97 / 0.001$ & $0.69 \pm 0.5$ & $0.02 * / 0.19$ & $0.82 \pm 0.5$ & $0.001 * / 0.41$ \\
\hline IRFD left ex (N/ms) (SG) & $0.38 \pm 0.3$ & & $0.34 \pm 0.1$ & & $0.29 \pm 0.1$ & \\
\hline IRFD right flex (N/ms) (IG) & $0.13 \pm 0.1$ & $0.14 / 0.08$ & $0.25 \pm 0.1$ & $0.01 * / 0.23$ & $0.39 \pm 1.5$ & $<0.001 * / 0.64$ \\
\hline IRFD right flex (N/ms) (SG) & $0.19 \pm 0.1$ & & $0.15 \pm 0.1$ & & $0.15 \pm 0.1$ & \\
\hline IRFD left flex (N/ms) (IG) & $0.17 \pm 0.1$ & $0.97 / 0.001$ & $0.26 \pm 0.2$ & $0.02 * / 0.19$ & $0.39 \pm 0.2$ & $<0.001 * / 0.42$ \\
\hline IRFD left flex (N/ms) (SG) & $0.15 \pm 0.1$ & & $0.14 \pm 0.1$ & & $0.15 \pm 0.1$ & \\
\hline
\end{tabular}

Abbreviation: IG: intervention group, SG: sham group, SPPB: Short Physical Performance Battery, IMVC: isometric maximal voluntary contraction, ex: extension, flex: flexion, $\mathrm{N}$ : Newton, IRFD: isometric rate of force development, N/ms: NewtoN/ mseconds, ${ }^{\circ}$ : significant difference between groups $p<0.05,{ }^{*}$ : significant difference between groups after Bonferroni correction $p<0.025, \eta^{2}$ : effect size: $\eta^{2}=.01$; small effect, $\eta^{2}=.06$; moderate effect, $\eta^{2}=.14$; large effect 


\section{Discussion}

This study aimed to assess the effects of SR-WBV \& Video Dance Game Training that was accompanied with motivational instructions in LTC elderly on lower extremity physical function and leg muscle properties. We hypothesised that an intervention program that targets motor control will effect on physical functioning and muscle strength levels of LTC elderly. The results of the study demonstrate that a combination of SR-WBV and DVG may be used as a skillingup exercise for LTC elderly because of significant SPPB score change values in IG (+58.8\%) compared to SG (- 4.0\%) and concomitant significant strength improvements seen in Fsub, IRFD, and IRFDsub in IG compared to SG.

Coaching the LTC participants in both groups with a professional exercise instructor to enhance exercise participation, aimed to ensure that the targeted exercise frequencies and levels would be reached, and to prevent attrition. This approach showed to be rather succesfull. We demonstrated the feasibility of a motivational approach through high adherence rates for LTC dwelling older people randomised in this clinical trial. Our target of $75 \%$ compliance for the 8-weeks training project was by far attained. Furthermore, no individuals were considered noncompliant for the training. Thus, compliance with the exercise interventions and retesting was excellent. Compared with median rates for recruitment, attrition and adherence in falls prevention interventions in institutional settings for clinical trials [82] we achieved better rates. However, we report on values after 8 training weeks. Nyman and Victor [82] and Fuchs and colleagues [64] report values that may be expected by 12 months. In future trials with LTC individuals the follow-up period for the assessment of adherence and attrition should, therefore, preferably be extended to a similar time frame to facilitate comparability with reference values.

Previous studies in elderly individuals have referred to the usefulness of WBV training on both muscle strength [83-87] and balance [88-91]. Playing certain types of Video Games had an effect on muscle strength [92], balance [93], and gait [94]. However, few studies found results with similar high effect sizes as this study. The combination of SR-WBV and DVG might, therefore, be more effective in activating the sensorimotor system compared to published studies that investigated solely training using one of these approaches [51, 83, 92-95]. However, future studies that compare both approaches against each other are needed to substantiate or refute this assumption. It is known that traditional strengthening improves muscle strength as a result of an improved neural drive and muscle hypertrophy [96-98]. Gruber and Gollhofer [72] postulated that sensorimotor training had a large influence on the neuromuscular system at the initiation of production of rate of force development and neuromuscular activation at the onset of voluntary actions. The motoneuron outputs induced by sensorimotor training comprise elevated central motor drive, motoneuron recruitment or firing frequency, alterations in synchronisation of motor unit firing, and reduced presynaptic inhibition $[72,99]$. However, the increase in IRFD after sensorimotor training is not associated with an increase in maximum voluntary contraction [72]. As a sensorimotor training method, SR-WBV and DVG seems, therefore, to mainly affect the neural drive. 
The results of the present study potentially have important functional consequences. Age-related degenerative processes, referred to as dynapenia, are considered a contributing factor to loss of independence in daily living [20]. High RFD is important in various activities of daily life where a sudden strength capacity is required, and to counteract sudden perturbations, e.g. in postural control to avoid falls $[98,100]$. A typical contraction time involved in such movements is between 50 to $250 \mathrm{~ms}$. In contrast, the time to reach maximum strength in most human muscles is over 300 ms, e.g. for knee extensors [101]. On the basis of the previously described reasoning it seems plausible that IMVC did not significantly change during sensorimotor training. However, physical performance improved significantly. RFD is more closely related to physical performance than IMVC [102, 103]. The result of this study is in accordance with Bottaro et al. [104], who were able to find an increase in RFD in parallel with an improvement in physical performance.

This study presents a mean change after four weeks of about 2.7 points and after eight weeks of 4.2 points on the SPPB scale in the IG. After four weeks the SG shows a mean change of about -0.5 and after eight weeks a mean change -0.2 points on the SPPB scale. Changes of about 1 point on the SPPB scale are substantial [105]. From a clinical standpoint, low SPPB point scores have a predictive value in activities in daily living [69], loss of mobility [106], admission to nursing facilities, disability [66, 69], hospitalization [107] and mortality [108]. In addition, physical performance measures have been used to test the efficacy of preventive strategies [109]. An improvement on the SPPB point scale through SR-WBV and DVG may reduce the risk of major mobility disability. Maintaining mobility is a central component in sustaining independence in daily living. Drey et al. [105] described that muscle strength and muscle power during follow up interventions have been shown to be equally beneficial for increasing physical function in elderly individuals. Future studies should be designed with adequate follow up measures to further investigate and record the possible impact of SR-WBV and DVG on the activities of daily living.

An additional advantage of this current study over previous WBV or DVG investigations in the elderly is the use of the classification system defined by Zeyfang and Braun [110]. Elderly individuals are not a homogeneous group. There are biologically elderly individuals who still feel young at heart, having a high physical fitness and performance level, and anticipating a few decades of life expectancy ahead of them. On the other spectrum frail elderly can be situated. Therefore, functional status of participating older individuals should be emphasized in training studies. To manage elderly individuals' needs in consideration of diagnostic or treatment goals or maintenance of health, the framework "Go-Go, Slow-Go and No-Go" could be used. This classification was introduced by Zeyfang and Braun [110] classifying older adults as "being an independent person" (Go-Go); "being a needy person with a slight handicap" (Slow-Go); and "being a person in need of care with severe functional limitation" (No-Go). The need for care may be defined as depending permanently on assistance (No-Go) or depending on support in everyday activities such as dressing, body care, eating, using the toilet, mobility, and planning the day (Slow-Go) [111]. A systematic review indicated that older adults categorized in these 
groups react differently when provided with the same training stimuli [38].Our study reflects the need to consider the functional status of the included participants. Bautmans et al. [88] included all residents in a nursing home within dependence categories "O"(do not need assistance), "A" (need assistance in two ADLs: washing and dressing) and "B" (require assistance in three ADLs) according to the scale of Katz et al. [112]. When their participants are categorised with our classification it becomes apparent that only Go-Go and Slow-Go elderly were included. This finding is reflective of the observation that there are only few studies $[51,83,95,113]$ that have focused separately on the Go-Go, Slow-Go and No-Go classification of older individuals.

There are some limitations in this study that should be mentioned. It was a single blind study. Studies where the examiner is not blinded might be at a higher risk of attrition [114] or assessment bias [115]. Future studies should, therefore, try to replicate our findings using a design where the examiner is blinded. No long-lasting effects follow-up measurements were obtained on the impact of the program on functional performance, strength or fall rates. Future studies should carry out such follow-up measurements to evaluate lasting effects. Furthermore, we used performance (SPPB) and impairment level (lower body muscle strength) based measures as proxies for PA [116, 117]. Although these measures are correlated and randomised clinical trial intervention studies in older adults show that PA improves measures of physical performance $[109,118]$ future studies should include quantified measures of PA instead of proxy measures.

\section{Conclusions}

During SR-WBV and DVG intervention, three times a week within eight weeks physical performance and rate of force development improved significantly compared to sham intervention in No-Go (need-of-care) elderly residing in LTC. The results of this study indicate that SR-WBV and DVG can be used as a skilling up training regime for No-Go elderly individuals. Future investigations are warranted and should include follow-up measures, together with additional measures of functional strength, balance, gait and fall risk.

\section{Abbreviations}

ADLs: activity of daily living; ANOVA: analyses of varianc; DVG: dance video game; ES: effect size; Fsub: Submaximal force; IG: intervention group; Hz: hertz; IMVC: isometric voluntary contraction; IRFD: isometric rate of force development; IRFDsub: submaximal IRFD values, Longterm care: LTC, MMSE: Mini-Mental Status Examination; ms: millisseconds; N: Newton; N/ms: Newton/milliseconds; RAI: Resident Assessment Instrument; RFD: rate of force development; SH: sham group; SPPB: Short Physival Performance Battery; SR-WBV: stochastic resonance whole-body vibration; WBV: whole-body vibration 


\section{Competing interests}

The authors declare no potential conflict of interest with respect to the authorship or publication of this article.

\section{Authors' contributions}

SR and EdB conceived the idea of this study. SR, LR, HB, RB and EdB participated in the conception and design of the study. SR supervised the WBV trainings session and Data collection. $\mathrm{SR}$ and $\mathrm{EdB}$ conducted the statistical analyses. $\mathrm{SR}, \mathrm{RB}$ and $\mathrm{EdB}$ wrote the first manuscript version. $\mathrm{SR}, \mathrm{LR}, \mathrm{RH}, \mathrm{DS}, \mathrm{RdB}$, and $\mathrm{EdB}$ were involved in drafting or revising the manuscript. All authors read and approved the final manuscript.

\section{Acknowledgements}

We would like to thank Bennie Ross for proof reading and Patric Eichelberger for strength measurement data processing on Matlab. 


\section{References}

1. Rikli RE, Jones CJ: Development and validation of a functional fitness test for community-residing older adults. J Aging Phys Activ 1999, 7(2):129-161.

2. Fried LP, Ferrucci L, Darer J, Williamson JD, Anderson G: Untangling the concepts of disability, frailty, and comorbidity: Implications for improved targeting and care. J Gerontol a-Biol 2004, 59(3):255-263.

3. Markle-Reid M, Browne G: Conceptualizations of frailty in relation to older adults. J Adv Nurs 2003, 44(1):58-68.

4. Leveille, S. G., Wagner, E. H., Davis, C., Grothaus, L., Wallace, J., LoGerfo, M., \& Kent, D. Preventing disability and managing chronic illness in frail older adults: A randomized trial of a community based partnership with primary care. Journal of the American Geriatrics Society 1998, 46(10), 1191-1198

5. Baert V, Gorus E, Mets T, Geerts C, Bautmans l: Motivators and barriers for physical activity in the oldest old: a systematic review. Ageing research reviews 2011, 10(4):464-474

6. Freiberger E, Sieber C, Pfeifer K: Physical activity, exercise, and sarcopenia - future challenges. Wien Med Wochenschr 2011, 161(17-18):416-425.

7. Benjamin K, Edwards N, Ploeg J, Legault F: Barriers to physical activity and restorative care for residents in long-term care: a review of the literature. J Aging Phys Act 2014, 22(1):154-165.

8. De Souto Barreto P, Demougeot L, Vellas $B$, Rolland Y: How much exercise are older adults living in nursing homes doing in daily life? A cross-sectional study. Journal of sports sciences 2015, 33(2):116-124.

9. Roubenoff R, Hughes VA: Sarcopenia: current concepts. The journals of gerontology Series A, Biological sciences and medical sciences 2000, 55(12):M716724.

10. Rosenberg $\mathrm{H}$ : Sarcopenia: origins and clinical relevance. J Nutr 1997, 127(5 Suppl):990S-991S.

11. Clark BC, Fernhall B, Ploutz-Snyder LL: Adaptations in human neuromuscular function following prolonged unweighting: I. Skeletal muscle contractile properties and applied ischemia efficacy. J Appl Physiol 2006, 101(1):256-263.

12. Delmonico MJ, Harris TB, Visser M, Park SW, Conroy MB, Velasquez-Mieyer P, Boudreau R, Manini TM, Nevitt M, Newman AB et al: Longitudinal study of muscle strength, quality, and adipose tissue infiltration. Am J Clin Nutr 2009, 90(6):1579-1585.

13. Kawakami Y, Akima H, Kubo K, Muraoka Y, Hasegawa H, Kouzaki M, Imai M, Suzuki Y, Gunji A, Kanehisa $\mathrm{H}$ et al: Changes in muscle size, architecture, and neural activation after 20 days of bed rest with and without resistance exercise. Eur J Appl Physiol 2001, 84(1-2):7-12.

14. Clark BC, Manini TM, Bolanowski SJ, Ploutz-Snyder LL: Adaptations in human neuromuscular function following prolonged unweighting: II. Neurological properties and motor imagery efficacy. J Appl Physiol 2006, 101(1):264-272.

15. Clark BC, Pierce JR, Manini TM, PloutzSnyder LL: Effect of prolonged unweighting of human skeletal muscle on neuromotor force control. Eur J Appl Physiol 2007, 
100(1):53-62.

16. Clark BC: In vivo alterations in skeletal muscle form and function after disuse atrophy. Med Sci Sports Exerc 2009, 41(10):1869-1875

17. Clark BC, Issac LC, Lane JL, Damron LA, Hoffman RL: Neuromuscular plasticity during and following 3 wk of human forearm cast immobilization. J Appl Physiol 2008, 105(3):868-878

18. Clark BC, Taylor JL, Hoffman RL, Dearth DJ, Thomas JS: Cast immobilization increases long-interval intracortical inhibition. Muscle Nerve 2010, 42(3):363-372.

19. Hill AV: The mechanics of active muscle Proc Roy Soc Lond (Biol) 1953, 141:104117.

20. Clark BC, Manini TM: What is dynapenia? Nutrition 2012, 28(5):495-503.

21. Manini TM, Clark BC: Dynapenia and aging: an update. The journals of gerontology Series A, Biological sciences and medical sciences 2012, 67(1):28-40.

22. Duchateau J, Enoka RM: Neural adaptations with chronic activity patterns in able-bodied humans. American journal of physical medicine \& rehabilitation / Association of Academic Physiatrists 2002, 81(11 Suppl):S17-27.

23. Clark BC, Taylor JL: Age-related changes in motor cortical properties and voluntary activation of skeletal muscle. Current aging science 2011, 4(3):192-199.

24. Ward RE, Boudreau RM, Caserotti P, Harris TB, Zivkovic S, Goodpaster BH, Satterfield S, Kritchevsky SB, Schwartz AV, Vinik AI et al: Sensory and motor peripheral nerve function and incident mobility disability. J Am Geriatr Soc 2014, 62(12):2273-2279.

25. Ward RE, Boudreau RM, Caserotti P, Harris
TB, Zivkovic S, Goodpaster BH, Satterfield S, Kritchevsky S, Schwartz AV, Vinik Al et al: Sensory and Motor Peripheral Nerve Function and Longitudinal Changes in Quadriceps Strength. J Gerontol a-Biol 2015, 70(4):464-470.

26. Borghuis J, Hof AL, Lemmink KA: The importance of sensory-motor control in providing core stability: implications for measurement and training. Sports Med 2008, 38(11):893-916.

27. Comerford MJ, Mottram SL: Movement and stability dysfunction--contemporary developments. Manual therapy 2001, 6(1):15-26.

28. Scott SH: Optimal feedback control and the neural basis of volitional motor control. Nature reviews Neuroscience 2004, 5(7):532-546 .

29. Radebold A, Cholewicki J, Polzhofer GK, Greene HS: Impaired postural control of the lumbar spine is associated with delayed muscle response times in patients with chronic idiopathic low back pain. Spine 2001, 26(7):724-730.

30. McDonnell MD, Ward LM: The benefits of noise in neural systems: bridging theory and experiment. Nature reviews Neuroscience 2011, 12(7):415-426.

31. Moss F, Ward LM, Sannita WG: Stochastic resonance and sensory information processing: a tutorial and review of application. Clinical neurophysiology : official journal of the International Federation of Clinical Neurophysiology 2004, 115(2):267-281.

32. Collins JJ, Imhoff TT, Grigg P: Noiseenhanced tactile sensation. Nature 1996, 383(6603): 770.

33. Cordo P, Inglis JT, Verschueren S, Collins 
JJ, Merfeld DM, Rosenblum S, Buckley S, Moss F: Noise in human muscle spindles. Nature 1996, 383(6603):769-770.

34. Priplata A, Niemi J, Salen M, Harry J, Lipsitz LA, Collins JJ: Noise-enhanced human balance control. Physical review letters 2002, 89(23):238101.

35. Collins JJ, Priplata AA, Gravelle DC, Niemi J, Harry J, Lipsitz LA: Noise-enhanced human sensorimotor function. IEEE engineering in medicine and biology magazine : the quarterly magazine of the Engineering in Medicine \& Biology Society 2003, 22(2):7683.

36. Chanou K, Gerodimos V, Karatrantou K, Jamurtas A: Whole-body vibration and rehabilitation of chronic diseases: a review of the literature. Journal of sports science \& medicine 2012, 11(2):187-200.

37. Rogan S, Hilfiker R, Schenk A, Vogler A, Taeymans J: Effects of whole-body vibration with stochastic resonance on balance in persons with balance disability and falls history - a systematic review. Res Sports Med 2014, 22(3):294-313.

38. Rogan $\mathrm{S}$, de Bruin ED, Radlinger L, Joehr C, Wyss C, Stuck NJ, Bruelhart Y, de Bie RA, Hilfiker R: Effects of whole-body vibration on proxies of muscle strength in old adults: a systematic review and meta-analysis on the role of physical capacity level. European review of aging and physical activity : official journal of the European Group for Research into Elderly and Physical Activity 2015, 12:12.

39. Mendez-Balbuena I, Manjarrez E, SchulteMonting J, Huethe F, Tapia JA, HeppReymond MC, Kristeva R: Improved sensorimotor performance via stochastic resonance. The Journal of neuroscience the official journal of the Society for Neuroscience 2012, 32(36):12612-12618.

40. Rogan $S$, Hilfiker R, Schmid S, Radlinger L: Stochastic resonance whole-body vibration training for chair rising performance on untrained elderly: a pilot study. Archives of gerontology and geriatrics 2012, 55(2):468473.

41. Haas CT, Turbanski S, Kessler K, Schmidtbleicher D: The effects of random whole-body-vibration on motor symptoms in Parkinson's disease. NeuroRehabilitation 2006, 21(1):29-36.

42. Moss F, Milton JG: Medical technology: balancing the unbalanced. Nature 2003, 425(6961):911-912.

43. Elfering A, Arnold S, Schade V, Burger C, Radlinger L: Stochastic resonance wholebody vibration, musculoskeletal symptoms, and body balance: a worksite training study. Safety and health at work 2013, 4(3):149155.

44. de Bruin ED, Schoene D, Pichierri G, Smith ST: Use of virtual reality technique for the training of motor control in the elderly. Some theoretical considerations. Zeitschrift fur Gerontologie und Geriatrie 2010, 43(4):229234.

45. Pichierri G, Wolf P, Murer K, de Bruin ED: Cognitive and cognitive-motor interventions affecting physical functioning: a systematic review. BMC geriatrics 2011, 11:29.

46. Rogan S, Radlinger L, Schmidtbleicher D, de Bie RA, de Bruin ED: Preliminary inconclusive results of a randomised double blinded cross-over pilot trial in longterm-care dwelling elderly assessing the feasibility of stochastic resonance wholebody vibration. European Review of Aging and Physical Activity 2015, 12(5). 
47. Ferrucci L, Guralnik JM, Studenski S, Fried LP, Cutler GB, Jr., Walston JD: Designing randomized, controlled trials aimed at preventing or delaying functional decline and disability in frail, older persons: a consensus report. J Am Geriatr Soc 2004, 52(4):625-634.

48. Fairhall N, Langron C, Sherrington C, Lord SR, Kurrle SE, Lockwood K, Monaghan N, Aggar C, Gill L, Cameron ID: Treating frailty--a practical guide. BMC medicine 2011, 9:83.

49. Campbell MK, Piaggio G, Elbourne DR, Altman DG, Group C: Consort 2010 statement: extension to cluster randomised trials. Bmj 2012, 345:e5661.

50. Cruz-Jentoft AJ, Baeyens JP, Bauer JM, Boirie Y, Cederholm T, Landi F, Martin FC, Michel JP, Rolland Y, Schneider SM et al: Sarcopenia: European consensus on definition and diagnosis: Report of the European Working Group on Sarcopenia in Older People. Age and ageing 2010, 39(4):412-423.

51. Kessler J, Radlinger L, Baur H, Rogan $\mathrm{S}$ : Effect of stochastic resonance whole body vibration on functional performance in the frail elderly: A pilot study. Archives of gerontology and geriatrics 2014, 59(2):305311.

52. Ahlborg L, Andersson C, Julin P: Wholebody vibration training compared with resistance training: effect on spasticity, muscle strength and motor performance in adults with cerebral palsy. J Rehabil Med 2006, 38(5):302-308.

53. Rogan $S$, Radlinger $L$, Hilfiker $R$, Schmidtbleicher D, de Bie RA, de Bruin ED: Feasibility and effects of applying stochastic resonance whole-body vibration on untrained elderly: a randomized crossover pilot study. BMC geriatrics 2015,
15(25).

54. Kaut O, Allert N, Coch C, Paus S, Grzeska A, Minnerop M, Wullner U: Stochastic resonance therapy in Parkinson's disease. NeuroRehabilitation 2011, 28(4):353-358.

55. Kaut $\mathrm{O}$, Jacobi $\mathrm{H}$, Coch $\mathrm{C}$, Prochnicki $\mathrm{A}$, Minnerop $\mathrm{M}$, Klockgether $\mathrm{T}$, Wullner $\mathrm{U}$ : A randomized pilot study of stochastic vibration therapy in spinocerebellar ataxia. Cerebellum 2014, 13(2):237-242.

56. Elfering A, Thomann J, Schade V, Radlinger L: Stochastic resonance whole body vibration reduces musculoskeletal pain: A randomized controlled trial. World journal of orthopedics 2011, 2(12):116-120.

57. Pichierri G, Murer K, de Bruin ED: A cognitive-motor intervention using a dance video game to enhance foot placement accuracy and gait under dual task conditions in older adults: a randomized controlled trial. BMC geriatrics 2012, 12:74.

58. Pichierri G, Coppe A, Lorenzetti S, Murer K, de Bruin ED: The effect of a cognitive-motor intervention on voluntary step execution under single and dual task conditions in older adults: a randomized controlled pilot study. Clinical interventions in aging 2012, 7:175-184.

59. O'Keeffe K: Dancing Monkeys (Automated creation of step files for Dance Dance Revolution). PhD. London: Imperial College.; 2003.

60. Fraser SA, Elliott $\mathrm{V}$, de Bruin ED, Bherer $\mathrm{L}$, Dumoulin C: The Effects of Combining Videogame Dancing and Pelvic Floor Training to Improve Dual-Task Gait and Cognition in Women with Mixed-Urinary Incontinence. Games for health journal 2014, 3(3):172-178.

61. Eggenberger $\mathrm{P}$, Schumacher $\mathrm{V}$, Angst $\mathrm{M}$, 
Theill N, de Bruin ED: Does multicomponent physical exercise with simultaneous cognitive training boost cognitive performance in older adults? A 6-month randomized controlled trial with a 1-year follow-up. Clinical Interventions in Aging 2015, 10:1335-1349.

62. Zech A, Drey M, Freiberger E, Hentschke C, Bauer JM, Sieber CC, Pfeifer K: Residual effects of muscle strength and muscle power training and detraining on physical function in community-dwelling prefrail older adults: a randomized controlled trial. BMC geriatrics 2012, 12:68.

63. Resnick B: Functional performance of older adults in a long-term care setting. Clinical nursing research 1998, 7(3):230-246; discussion 246-239.

64. Fuchs R, Goehner W, Seelig H: Long-term effects of a psychological group intervention on physical exercise and health: the MoVo concept. Journal of physical activity \& health 2011, 8(6):794-803.

65. Fuchs R, Göhner W, Seelig H, Fleitz A, Mahler C, Schittich I: Lebensstilintegrierte sportliche Aktivität: Ergebnisse der MoVo-LISA Interventionsstudie. Bewegungstherapie und Gesundheitssport 2010, 26:270-276.

66. Guralnik JM, Simonsick EM, Ferrucci L, Glynn RJ, Berkman LF, Blazer DG, Scherr PA, Wallace RB: A short physical performance battery assessing lower extremity function: association with self-reported disability and prediction of mortality and nursing home admission. Journal of gerontology 1994, 49(2):M85-94.

67. Evans WJ: Functional outcomes for clinical trials in frail older persons: Time to be moving. J Gerontol a-Biol 2008, 63(2):160-
164.

68. Ostir GV, Volpato S, Fried LP, Chaves P, Guralnik JM: Reliability and sensitivity to change assessed for a summary measure of lower body function: results from the Women's Health and Aging Study. J Clin Epidemiol 2002, 55(9):916-921.

69. Guralnik JM, Ferrucci L, Pieper CF, Leveille SG, Markides KS, Ostir GV, Studenski S, Berkman LF, Wallace RB: Lower extremity function and subsequent disability: consistency across studies, predictive models, and value of gait speed alone compared with the short physical performance battery. The journals of gerontology Series A, Biological sciences and medical sciences 2000, 55(4):M221231.

70. Guralnik JM, Ferrucci L, Simonsick EM, Salive ME, Wallace RB: Lower-extremity function in persons over the age of 70 years as a predictor of subsequent disability. The New England journal of medicine 1995, 332(9):556-561.

71. Ferrucci L, Penninx BW, Leveille SG, Corti MC, Pahor M, Wallace R, Harris TB, Havlik RJ, Guralnik JM: Characteristics of nondisabled older persons who perform poorly in objective tests of lower extremity function. J Am Geriatr Soc 2000, 48(9):1102-1110.

72. Gruber M, Gollhofer A: Impact of sensorimotor training on the rate of force development and neural activation. European journal of applied physiology 2004, 92(1-2):98-105.

73. Mebes C, Amstutz A, Luder G, Ziswiler $\mathrm{HR}$, Stettler M, Villiger PM, Radlinger $\mathrm{L}$ : Isometric rate of force development, maximum voluntary contraction, and 
balance in women with and without joint hypermobility. Arthritis and rheumatism 2008, 59(11):1665-1669.

74. Kwon S, Perera S, Pahor M, Katula JA, King AC, Groessl EJ, Studenski SA: What is a meaningful change in physical performance? Findings from a clinical trial in older adults (the LIFE-P study). J Nutr Health Aging 2009, 13(6):538-544.

75. Perera S, Mody SH, Woodman RC, Studenski SA: Meaningful change and responsiveness in common physical performance measures in older adults. J Am Geriatr Soc 2006, 54(5):743-749.

76. Perera, S., Studenski, S., Newman, A., Simonsick, E., Harris, T., Schwartz, A., \& Visser, M. (2014). Are estimates of meaningful decline in mobility performance consistent among clinically important subgroups?(Health ABC Study). The Journals of Gerontology Series A: Biological Sciences and Medical Sciences. 2014, 69(10):1260-8.

77. Hollis S, Campbell F: What is meant by intention to treat analysis? Survey of published randomised controlled trials. Bmj 1999, 319(7211):670-674.

78. Puri, M. L., Sen, P. K. A class of rank order tests for a general linear hypothesis. The Annals of Mathematical Statistics. 1969 1325-1343..

79. Rogan S, Radlinger L, Schmidtbleicher D, de Bie RA, de Bruin ED: Preliminary inconclusive results of a randomised double blinded cross-over pilot trial in longterm-care dwelling elderly assessing the feasibility of stochastic resonance wholebody vibration. European review of aging and physical activity : official journal of the European Group for Research into Elderly and Physical Activity 2015, 12:5.

80. Thomas JR, Nelson JK, Thomas KT: A generalized rank-order method for nonparametric analysis of data from exercise science: a tutorial. Research quarterly for exercise and sport 1999, 70(1):11-23.

81. Cohen J: Statistical power analysis for the behavioral sciences, 2nd edn. NJ, USA: Erlbaum, Hillsdale; 1988.

82. Nyman SR, Victor CR: Older people's recruitment, sustained participation, and adherence to falls prevention interventions in institutional settings: a supplement to the Cochrane systematic review. Age and ageing 2011, 40(4):430-436.

83. Zhang L, Weng C, Liu M, Wang Q, Liu L, He Y: Effect of whole-body vibration exercise on mobility, balance ability and general health status in frail elderly patients: a pilot randomized controlled trial. Clinical rehabilitation 2014, 28(1):59-68.

84. Lau RW, Liao LR, Yu F, Teo T, Chung RC, Pang MY: The effects of whole body vibration therapy on bone mineral density and leg muscle strength in older adults: a systematic review and meta-analysis. Clinical rehabilitation 2011, 25(11):975-988.

85. Rogan S, Hilfiker R: [Training methods increase muscle strength due to whole-body vibration - force with $\mathrm{Hz}$ ]. Sportverletzung Sportschaden : Organ der Gesellschaft fur Orthopadisch-Traumatologische Sportmedizin 2012, 26(4):185-187.

86. Bogaerts A, Delecluse C, Claessens AL, Coudyzer W, Boonen S, Verschueren SM: Impact of whole-body vibration training versus fitness training on muscle strength and muscle mass in older men: a 1-year randomized controlled trial. The journals of 
gerontology Series A, Biological sciences and medical sciences 2007, 62(6):630-635

87. Machado A, Garcia-Lopez D, GonzalezGallego J, Garatachea N: Whole-body vibration training increases muscle strength and mass in older women: a randomizedcontrolled trial. Scandinavian journal of medicine \& science in sports 2010, 20(2):200-207.

88. Bautmans I, Van Hees E, Lemper JC, Mets T: The feasibility of Whole Body Vibration in institutionalised elderly persons and its influence on muscle performance, balance and mobility: a randomised controlled trial [ISRCTN62535013]. BMC geriatrics 2005, 5:17.

89. Rogan S, Hilfiker R, Herren K, Radlinger L, de Bruin ED: Effects of whole-body vibration on postural control in elderly: a systematic review and meta-analysis. BMC geriatrics 2011, 11:72.

90. Rogan S, Hilfiker R, Schenk A, Vogler A, Taeymans J: Effects of whole-body vibration with stochastic resonance on balance in persons with balance disability and falls history - a systematic review. Res Sports Med 2014;22:294-313.

91. Sitja-Rabert M, Martinez-Zapata MJ, Fort Vanmeerhaeghe A, Rey Abella F, RomeroRodriguez D, Bonfill X: Effects of a Whole Body Vibration (WBV) Exercise Intervention for Institutionalized Older People: A Randomized, Multicentre, Parallel, Clinical Trial. Journal of the American Medical Directors Association 2015, 16(2):125-131.

92. Jorgensen $M G$, Laessoe $U$, Hendriksen C, Nielsen OB, Aagaard P: Efficacy of Nintendo Wii training on mechanical leg muscle function and postural balance in community-dwelling older adults: a randomized controlled trial. The journals of gerontology Series A, Biological sciences and medical sciences 2013, 68(7):845-852.

93. Wuest $S$, Borghese NA, Pirovano $M$, Mainetti $\mathrm{R}$, van de Langenberg $\mathrm{R}$, de Bruin ED: Usability and Effects of an ExergameBased Balance Training Program. Games for health journal 2014, 3(2):106-114.

94. De Bruin E, Reith A, M. D, Murer K: Feasibility of Strength-Balance Training Extended with Computer Game Dancing in Older People; Does it Affect Dual Task Costs of Walking? J Nov Physiother 2011, 1(104).

95. Rogan S, Schmidtbleicher D, Radlinger L: Immediate effects after stochastic resonance whole-body vibration on physical performance on frail elderly for skilling-up training: a blind cross-over randomised pilot study. Aging clinical and experimental research 2014.26(5):519-27.

96. Sale DG: Neural adaptation to resistance training. Medicine and science in sports and exercise 1988, 20(5 Suppl):S135-145.

97. Schmidtbleicher D, Haralambie G: Changes in contractile properties of muscle after strength training in man. European journal of applied physiology and occupational physiology 1981, 46(3):221-228.

98. Aagaard P, Simonsen EB, Andersen JL, Magnusson P, Dyhre-Poulsen P: Increased rate of force development and neural drive of human skeletal muscle following resistance training. Journal of applied physiology 2002, 93(4):1318-1326.

99. Aagaard P: Training-induced changes in neural function. Exercise and sport sciences reviews 2003, 31(2):61-67.

100. Aagard P, Mayer F: Neuronal adaption to strength training. Deutsche Zeitschrift für Sportmedizin 2007, 58(2):50-53. 
101. Thorstensson A, Karlsson J, Viitasalo JH, Luhtanen P, Komi PV: Effect of strength training on EMG of human skeletal muscle. Acta physiologica Scandinavica 1976, 98(2):232-236.

102. Bean JF, Leveille SG, Kiely DK, Bandinelli S, Guralnik JM, Ferrucci L: A comparison of leg power and leg strength within the InCHIANTI study: which influences mobility more? The journals of gerontology Series A, Biological sciences and medical sciences 2003, 58(8):728-733.

103. Foldvari M, Clark M, Laviolette LC, Bernstein MA, Kaliton D, Castaneda C, Pu CT, Hausdorff JM, Fielding RA, Singh MA: Association of muscle power with functional status in community-dwelling elderly women. The journals of gerontology Series A, Biological sciences and medical sciences 2000, 55(4):M192-199.

104. Bottaro M, Machado SN, Nogueira W, Scales R, Veloso J: Effect of high versus low-velocity resistance training on muscular fitness and functional performance in older men. European journal of applied physiology 2007, 99(3):257-264.

105. Drey $M$, Zech A, Freiberger E, Bertsch T, Uter W, Sieber CC, Pfeifer K, Bauer $\mathrm{JM}$ : Effects of strength training versus power training on physical performance in prefrail community-dwelling older adults. Gerontology 2012, 58(3):197-204.

106. Studenski S, Perera S, Wallace D, Chandler JM, Duncan PW, Rooney E, Fox M, Guralnik JM: Physical performance measures in the clinical setting. Journal of the American Geriatrics Society 2003, 51(3):314-322.

107. Penninx BW, Ferrucci L, Leveille SG, Rantanen T, Pahor M, Guralnik JM: Lower extremity performance in nondisabled older persons as a predictor of subsequent hospitalization. The journals of gerontology Series A, Biological sciences and medical sciences 2000, 55(11):M691-697.

108. Ostir GV, Kuo YF, Berges IM, Markides KS, Ottenbacher KJ: Measures of lower body function and risk of mortality over 7 years of follow-up. American journal of epidemiology 2007, 166(5):599-605.

109. Investigators LS, Pahor M, Blair SN, Espeland M, Fielding R, Gill TM, Guralnik JM, Hadley EC, King AC, Kritchevsky SB et al: Effects of a physical activity intervention on measures of physical performance: Results of the lifestyle interventions and independence for Elders Pilot (LIFE-P) study. The journals of gerontology Series A, Biological sciences and medical sciences 2006, 61(11):1157-1165.

110. Zeyfang A, Braun A: [Guidelines "Diabetes mellitus in the elderly"]. MMW Fortschritte der Medizin 2009, 151(20):33-35, 37.

111. Swiss Academy Of $\mathrm{M}$ : Treatment and care of elderly persons who are in need of care. Swiss medical weekly 2004, 134(4142):618-626.

112. Katz S, Ford AB, Moskowitz RW, Jackson BA, Jaffe MW: Studies of Illness in the Aged. The Index of Adl: A Standardized Measure of Biological and Psychosocial Function. Jama 1963, 185:914-919.

113. Sievanen H, Karinkanta S, Moisio-Vilenius P, Ripsaluoma J: Feasibility of whole-body vibration training in nursing home residents with low physical function: a pilot study. Aging clinical and experimental research 2014, 26(5):511-517.

114. Juni P, Altman DG, Egger M: Systematic reviews in health care: Assessing the quality of controlled clinical trials. Bmj 2001, 
323(7303):42-46.

115. Kaptchuk TJ: Effect of interpretive bias on research evidence. Bmj 2003, 326(7404):1453-1455.

116. Chale-Rush A, Guralnik JM, Walkup MP, Miller ME, Rejeski WJ, Katula JA, King AC, Glynn NW, Manini TM, Blair SN et al: Relationship between physical functioning and physical activity in the lifestyle interventions and independence for elders pilot. J Am Geriatr Soc 2010, 58(10):19181924.

117. Leblanc A, Pescatello LS, Taylor BA, Capizzi JA, Clarkson PM, Michael White C, Thompson PD: Relationships between physical activity and muscular strength among healthy adults across the lifespan. SpringerPlus 2015, 4:557.

118. Cress ME, Buchner DM, Questad KA, Esselman PC, deLateur BJ, Schwartz RS: Exercise: effects on physical functional performance in independent older adults. J Gerontol A Biol Sci Med Sci 1999, 54(5):M242-248. 


\section{Additional material}

\section{Addional file 1}

ANOVA with repeated measurements (ranks) intergroup-by-time effects and group-by-time interaction for the secondary outcomes Fsub 30ms (N)

\begin{tabular}{lllll}
\hline & $\begin{array}{l}\text { Pillai 's trace } \\
\left(\mathbf{r}^{2}=\mathbf{S S}_{\text {Bet }} / \mathbf{S S}_{\text {Tot }}\right)\end{array}$ & $\begin{array}{l}\mathbf{L} \\
{\left[(\mathbf{N}-\mathbf{1}) \mathbf{r}^{2}\right]}\end{array}$ & $\mathbf{p}$ & ES $\left(\mathbf{n}^{2}\right)$ \\
\hline Fsub 30ms right ex (N) (time effects) & 0.14 & 1.90 & 0.171 & 0.14 \\
Fsub 30ms right ex (N) (interaction effects) & 0.29 & 4.85 & $0.02^{*}$ & 0.29 \\
Fsub 30ms left ex (N) (time effects) & 0.01 & 0.008 & 0.99 & 0.001 \\
Fsub 30ms left ex (N) (interaction effects) & 0.35 & 7.62 & $0.002^{*}$ & 0.35 \\
Fsub 30ms right flex (N) (time effects) & 0.001 & 0.002 & 0.97 & 0.001 \\
Fsub 30ms right flex (N) (interaction effects) & 0.14 & 2.30 & 0.12 & 0.14 \\
Fsub 30ms left flex (N) (time effects) & 0.001 & 0.001 & 1.00 & 0.001 \\
Fsub 30ms left flex (N) (interaction effects) & 0.14 & 1.80 & 0.18 & 0.14 \\
\hline
\end{tabular}

Legend: Fsub: Submaximal force; ${ }^{\circ}$ : significant difference $\mathrm{p}<0.05{ }^{*}$ : : siginificant difference after Bonferroni adjustment $\mathrm{p}<0.0125$; ES: effect size ( $\eta 2=.01$; small effect, $\eta 2=.06$; moderate effect, $\eta 2=.14$; large effect)

\section{Additional file 2}

Outcome Fsub 30ms (N) data and between group comparison at BASE, $4 \mathrm{~W}$ and $8 \mathrm{~W}$

\begin{tabular}{|c|c|c|c|c|c|c|}
\hline & BASE & $p / \eta^{2}$ & $4 W$ & $p / \eta^{2}$ & $8 W$ & $p / \eta^{2}$ \\
\hline Fsub 30ms right ex (N) (IG) & $152.4 \pm 55.1$ & $0.78 / 0.003$ & $138.5 \pm 58$ & $0.86 / 0.001$ & $220.5 \pm 58$ & $0.022 * / 019$ \\
\hline Fsub 30ms right ex (N/) (SG) & $166.5 \pm 0.2$ & & $134.6 \pm 64$ & & $153.5 \pm 64.1$ & \\
\hline Fsub 30ms left ex (N) (IG) & $140.6 \pm 89$ & $0.97 / 0.001$ & $163.4 \pm 78$ & $0.14 / 0.08$ & $195.0 \pm 90$ & $0.003 * / 0.26$ \\
\hline Fsub 30ms left ex (N) (SG) & $129.8 \pm 55$ & & $119.7 \pm 52$ & & $121.6 \pm 66$ & \\
\hline Fsub 30ms right flex (N) (IG) & $61.3 \pm 29$ & $0.88 / 0.001$ & $74.7 \pm 31$ & $0.29 / 0.04$ & $180.6 \pm 72$ & $0.97 / 0.09$ \\
\hline Fsub $30 \mathrm{~ms}$ right flex (N) (SG) & $64.3 \pm 30.9$ & & $76.2 \pm 32$ & & $141.7 \pm 72$ & \\
\hline Fsub 30ms left flex (N) (IG) & $73.7 \pm 41$ & $0.39 / 0.03$ & $79.3 \pm 43$ & $0.16 / 0.07$ & $87.3 \pm 35$ & $0.02 * / 0.18$ \\
\hline Fsub 30ms left flex (N) (SG) & $58.8 \pm 21$ & & $61.8 \pm 22$ & & $61.3 \pm 19$ & \\
\hline
\end{tabular}

Legend: Fsub: Submaximal force, N: Newton; IG: intervention group, SG: sham group, p: between groups, ex: extension, flex: felxion, ms: milisecond, ${ }^{\circ}:$ significant difference $p<0.05$, *: siginificant difference after Bonferroni correction $p<0.025$, $\eta^{2}$ : effect size: $\eta^{2}=.01$; small effect, $\eta^{2}=.06$; moderate effect, $\eta^{2}=.14$; large effect 


\section{Addional file 3}

ANOVA with repeated measurements (ranks) intergroup-by-time effects and group-by-time interaction for the secondary outcomes Fsub $50 \mathrm{~ms}(\mathrm{~N})$

\begin{tabular}{lllll}
\hline & $\begin{array}{l}\text { Pillai's trace } \\
\left(\mathbf{r}^{2}=\mathbf{S S}_{\text {Bet }} \mathbf{S S}_{\text {Tot }}\right)\end{array}$ & $\begin{array}{l}\mathbf{L} \\
{\left[(\mathbf{N}-\mathbf{1}) \mathbf{r}^{\mathbf{2}}\right]}\end{array}$ & $\mathbf{p}$ & $\mathbf{E S}\left(\mathbf{n}^{\mathbf{2}}\right)$ \\
\hline Fsub 50ms right ex (N) (time effects) & 0.001 & 0.006 & 0.994 & 0.001 \\
Fsub 50ms right ex (N) (interaction effects) & 0.28 & 5.41 & $0.01^{\circ}$ & 0.28 \\
Fsub 50ms left ex (N) (time effects) & 0.09 & 1.12 & 0.34 & 0.09 \\
Fsub 50ms left ex (N) (interaction effects) & 0.33 & 5.96 & $0.008^{\circ}$ & 0.33 \\
Fsub 50ms right flex (N) (time effects) & 0.001 & 0.001 & 1.00 & 0.001 \\
Fsub 50ms right flex (N) (interaction effects) & 0.06 & 0.96 & 0.39 & 0.06 \\
Fsub 50ms left flex (N) (time effects) & 0.001 & 0.003 & 1.00 & 0.001 \\
Fsub 50ms left flex (N) (interaction effects) & 0.19 & 3.17 & 0.06 & 0.19 \\
\hline
\end{tabular}

Legend: Fsub: Submaximal force; ${ }^{\circ}$ : significant difference $p<0.05,{ }^{*}$ : siginificant difference after Bonferroni adjustment $p$ $<0.0125$; ES: effect size $(\eta 2=.01 ;$ small effect, $\eta 2=.06 ;$ moderate effect, $\eta 2=.14$; large effect $)$

\section{Additional file 4}

Outcome values af Fsub 50ms $(\mathrm{N})$ data and between group comparison at BASE, $4 \mathrm{~W}$ and $8 \mathrm{~W}$

\begin{tabular}{lllllll}
\hline & BASE & $\mathbf{p} / \mathbf{n}^{2}$ & $\mathbf{4 W}$ & $\mathbf{p} / \mathbf{n}^{2}$ & $\mathbf{8 W}$ & $\mathbf{p} / \mathbf{n}^{2}$ \\
\hline Fsub 50ms right ex (N) (IG) & $136.2 \pm 58$ & $0.616 / 0.01$ & $138.7 \pm 56$ & $0.55 / 0.817$ & $180.7 \pm 72$ & $0.09 / 0.09$ \\
Fsub 50ms right ex (N/) (SG) & $133.1 \pm 75$ & & $134.6 \pm 57$ & & $141.7 \pm 65.2$ & \\
Fsub 50ms left ex (N) (IG) & $161.5 \pm 88$ & $0.962 / 0.001$ & $163.1 \pm 81$ & $0.23 / 0.06$ & $195.2 \pm 93$ & $0.01^{*} / 0.22$ \\
Fsub 50ms left ex (N) (SG) & $153.6 \pm 56$ & & $119.8 \pm 59$ & & $120.5 \pm 74$ & \\
Fsub 50ms right flex (N) (IG) & $61.3 \pm 29$ & $0.39 / 0.25$ & $87.5 \pm 38$ & $0.15 / 0.07$ & $138.8 \pm 56$ & $0.84 / 0.001$ \\
Fsub 50ms right flex (N) (SG) & $64.4 \pm 31$ & & $65.8 \pm 25$ & & $65.8 \pm 25$ & \\
Fsub 50ms left flex (N) (IG) & $73.7 \pm 41$ & $0.88 / 0.001$ & $79.4 \pm 43$ & $0.08 / 0.11$ & $139.4 \pm 56$ & $0.182 / 0.002$ \\
Fsub 50ms left flex (N) (SG) & $58.8 \pm 21$ & & $61.9 \pm 22$ & & $135 \pm 57$ & \\
\hline
\end{tabular}

Legend: Fsub: Submaximal force, N: Newton; IG: intervention group, SG: sham group, p: between groups, ex: extension, flex: felxion, ms: milisecond, ${ }^{\circ}:$ significant difference $p<0.05$, *: siginificant difference after Bonferroni correction $p<0.025$, $\eta^{2}$ : effect size: $\eta^{2}=.01 ;$ small effect, $\eta^{2}=.06$; moderate effect, $\eta^{2}=.14$; large effect 


\section{Addional file 5}

ANOVA with repeated measurements (ranks) intergroup-by-time effects and group-by-time interaction for the secondary outcomes Fsub $100 \mathrm{~ms}(\mathrm{~N})$

\begin{tabular}{lllll}
\hline & $\begin{array}{l}\text { Pillai's trace } \\
\left(\mathbf{r}^{2}=\mathbf{S S}_{\text {Bet }} / \mathbf{S S}_{\text {Tot }}\right)\end{array}$ & $\begin{array}{l}\mathbf{L} \\
{\left[(\mathbf{N}-\mathbf{1}) \mathbf{r}^{2}\right]}\end{array}$ & $\mathbf{p}$ & ES $\left(\mathbf{n}^{2}\right)$ \\
\hline Fsub 100ms right ex (N) (time effects) & 0.001 & 0.006 & 0.995 & 0.001 \\
Fsub 100ms right ex (N) (interaction effects) & 0.27 & 5.29 & $0.01^{*}$ & 0.27 \\
Fsub 100ms left ex (N) (time effects) & 0.001 & 0.008 & 0.99 & 0.001 \\
Fsub 100ms left ex (N) (interaction effects) & 0.36 & 7.36 & $0.003^{*}$ & 0.34 \\
Fsub 100ms right flex (N) (time effects) & 0.81 & 38.77 & $0.001^{*}$ & 0.81 \\
Fsub 100ms right flex (N) (interaction effects) & 0.20 & 2.29 & 0.10 & 0.20 \\
Fsub 100ms left flex (N) (time effects) & 0.820 & 40.98 & $0.001^{*}$ & 0.82 \\
Fsub 100ms left flex (N) (interaction effects) & 0.225 & 2.61 & 0.07 & 0.23 \\
\hline
\end{tabular}

Legend: Fsub: Submaximal force; ${ }^{\circ}$ : significant difference $p<0.05,{ }^{*}$ : siginificant difference after Bonferroni adjustment $p$ $<0.0125 ;$ ES: effect size $(\eta 2=.01 ;$ small effect, $\eta 2=.06 ;$ moderate effect, $\eta 2=.14$; large effect $)$

\section{Additional file 6}

Outcome values af Fsub 100ms ( $\mathrm{N}$ ) data and between group comparison at BASE, $4 \mathrm{~W}$ and $8 \mathrm{~W}$

\begin{tabular}{lllllll}
\hline & BASE & $\mathbf{p} / \mathbf{n}^{2}$ & $\mathbf{4 W}$ & $\mathbf{p} / \mathbf{\eta}^{2}$ & $\mathbf{8 W}$ & $\mathbf{p} / \mathbf{\eta}^{2}$ \\
\hline Fsub 100ms right ex (N) (IG) & $136.3 \pm 58$ & $0.62 / 0.009$ & $138.8 \pm 56$ & $0.82 / 0.002$ & $180.7 \pm 72$ & $0.08 / 0.09$ \\
Fsub 100ms right ex (N/) (SG) & $153.2 \pm 0.2$ & & $134.7 \pm 56$ & & $141.8 \pm 65$ & \\
Fsub 100ms left ex (N) (IG) & $140.8 \pm 89$ & $0.97 / 0.001$ & $163.6 \pm 78$ & $0.15 / 0.08$ & $195.2 \pm 90$ & $0.003^{*} / 0.26$ \\
Fsub 100ms left ex (N) (SG) & $130.0 \pm 55$ & & $119.9 \pm 52$ & & $121.8 \pm 66$ & \\
Fsub 100ms right flex (N) (IG) & $61.4 \pm 29$ & $0.88 / 0.001$ & $87.6 \pm 38$ & $0.08 / 0.11$ & $138.9 \pm 56$ & $0.82 / 0.02$ \\
Fsub 100ms right flex (N) (SG) & $64.5 \pm 31$ & & $65.9 \pm 25$ & & $65.9 \pm 25$ & \\
Fsub 100ms left flex (N) (IG) & $73.8 \pm 41.1$ & $0.39 / 0.03$ & $79.4 \pm 43$ & $0.16 / 0.07$ & $87.4 \pm 35$ & $0.01^{*} / 0.19$ \\
Fsub 100ms left flex (N) (SG) & $58.9 \pm 21$ & & $62.0 \pm 21$ & & $61.4 \pm 19$ & \\
\hline
\end{tabular}

Legend: Fsub: Submaximal force, N: Newton; IG: intervention group, SG: sham group, p: between groups, ex: extension, flex: flexion, ms: milisecond, ${ }^{\circ}$ : significant difference $p<0.05$, *: siginificant difference after Bonferroni correction $p<0.025$, $\eta^{2}$ : effect size: $\eta^{2}=.01$; small effect, $\eta^{2}=.06$; moderate effect, $\eta^{2}=.14$; large effect 


\section{Addional file 7}

ANOVA with repeated measurements (ranks) intergroup-by-time effects and group-by-time interaction for the secondary outcomes Fsub 200ms (N)

\begin{tabular}{lllll}
\hline & $\begin{array}{l}\text { Pillai 's trace } \\
\left(\mathbf{r}^{2}=\mathbf{S S}_{\text {Bet }} / \mathbf{S S}_{\text {Tot }}\right)\end{array}$ & $\begin{array}{l}\mathbf{L} \\
{\left[(\mathbf{N}-\mathbf{1}) \mathbf{r}^{\mathbf{2}}\right]}\end{array}$ & $\mathbf{p}$ & $\mathbf{E S}\left(\mathbf{n}^{2}\right)$ \\
\hline Fsub 200ms right ex (N) (time effects) & 0.001 & 0.006 & 1.00 & 0.001 \\
Fsub 200ms right ex (N) (interaction effects) & 0.28 & 2.00 & $0.01^{*}$ & 0.28 \\
Fsub 200ms left ex (N) (time effects) & 0.001 & 0.009 & 0.99 & 0.001 \\
Fsub 200ms left ex (N) (interaction effects) & 0.37 & 8.35 & $0.001^{*}$ & 0.37 \\
Fsub 200ms right flex (N) (time effects) & 0.001 & 0.003 & 0.997 & 0.001 \\
Fsub 200ms right flex (N) (interaction effects) & 0.15 & 2.49 & 0.15 & 0.15 \\
Fsub 200ms left flex (N) (time effects) & 0.001 & 0.002 & 1.00 & 0.001 \\
Fsub 200ms left flex (N) (interaction effects) & 0.11 & 1.74 & 0.19 & 0.11 \\
\hline
\end{tabular}

Legend: Fsub: Submaximal force; ${ }^{\circ}$ : significant difference $\mathrm{p}<0.05,{ }^{*}$ : siginificant difference after Bonferroni adjustment $\mathrm{p}$ $<0.0125$; ES: effect size $(\eta 2=.01 ;$ small effect, $\eta 2=.06 ;$ moderate effect, $\eta 2=.14$; large effect $)$

\section{Additional file 8}

Outcome values af Fsub 200ms (N) data and between group comparison at BASE, $4 \mathrm{~W}$ and $8 \mathrm{~W}$

\begin{tabular}{|c|c|c|c|c|c|c|}
\hline & BASE & $p / \eta^{2}$ & $4 \mathrm{~W}$ & $p / \eta^{2}$ & $8 W$ & $p / \eta^{2}$ \\
\hline Fsub 200ms right ex (N) (IG) & $136.5 \pm 17$ & $0.61 / 0.009$ & $139.0 \pm 14$ & $0.82 / 0.02$ & $180.8 \pm 17$ & $0.10 / 0.09$ \\
\hline Fsub 200ms right ex (N/) (SG) & $153.4 \pm 17$ & & $134.9 \pm 15$ & & $142.0 \pm 17$ & \\
\hline Fsub 200ms left ex (N) (IG) & $61.5 \pm 29$ & $0.88 / 0.001$ & $163.7 \pm 78$ & $0.14 / 0.08$ & $195.3 \pm 90$ & $0.003 * / 0.26$ \\
\hline Fsub 200ms left ex (N) (SG) & $64.6 \pm 31$ & & $120.0 \pm 52$ & & $121.9 \pm 66$ & \\
\hline Fsub 200ms right flex (N) (IG) & $61.5 \pm 29$ & $0.88 / 0.001$ & $75.0 \pm 31$ & $0.29 / 0.04$ & $87.7 \pm 38$ & $0.08 / 0.12$ \\
\hline Fsub 200ms right flex (N) (SG) & $64.6 \pm 0.1$ & & $67.5 \pm 32$ & & $66.0 \pm 25$ & \\
\hline Fsub 200ms left flex (N) (IG) & $73.9 \pm 41$ & $0.39 / 0.03$ & $79.4 \pm 43$ & $0.15 / 0.07$ & $87.5 \pm 36$ & $<0.019 * / 0.18$ \\
\hline Fsub 200ms left flex (N) (SG) & $59.7 \pm 21.7$ & & $62.1 \pm 22$ & & $61.5 \pm 19$ & \\
\hline
\end{tabular}

Legend: Fsub: Submaximal force, N: Newton; IG: intervention group, SG: sham group, p: between groups, ex: extension, flex: flexion, ms: milisecond, ${ }^{\circ}$ : significant difference $p<0.05$, *: siginificant difference after Bonferroni correction $p<0.025$, $\eta^{2}$ : effect size: $\eta^{2}=.01 ;$ small effect, $\eta^{2}=.06$; moderate effect, $\eta^{2}=.14$; large effect 


\section{Additional file 9}

ANOVA with repeated measurements (ranks) intergroup-by-time effects and group-by-time interaction for the secondary outcomes IRFDsub 0-30ms (N/ms)

\begin{tabular}{|c|c|c|c|c|}
\hline & $\begin{array}{l}\text { Pillai's trace } \\
\left(\mathrm{r}^{2}=\mathrm{SS}_{\mathrm{Bet}} / \mathrm{SS}_{\mathrm{Tot}}\right)\end{array}$ & $\begin{array}{l}\mathrm{L} \\
{\left[(\mathrm{N}-1) \mathrm{r}^{2}\right]}\end{array}$ & $\mathrm{p}$ & $E S\left(n^{2}\right)$ \\
\hline IRFDsub 0-30ms right ex (N/ms)(time effects) & 0.002 & 0.03 & 0.98 & 0.002 \\
\hline IRFDsub 0-30ms right ex (N/ms) (interaction effects) & 0.15 & 2.07 & 0.15 & 0.15 \\
\hline IRFDsub 0-30ms left ex (N/ms) (time effects) & 0.01 & 0.17 & 0.85 & 0.01 \\
\hline IRFDsub 0-30ms left ex (N/ms) (interaction effects) & 0.53 & 13.37 & $0.001^{*}$ & 0.53 \\
\hline IRFDsub 0-30ms right flex (N/ms) (time effects) & 0.006 & 0.07 & 0.94 & 0.006 \\
\hline IRFDsub 0-30ms right flex ( $\mathrm{N} / \mathrm{ms}$ ) (interaction effects) & 0.311 & 5.41 & $0.01^{*}$ & 0.31 \\
\hline IRFDsub 0-30ms left flex (N/ms) (time effects) & 0.01 & 0.12 & 0.89 & 0.01 \\
\hline IRFDsub 0-30ms left flex (N/ms) (interaction effects) & 0.45 & 9.78 & $0.001^{*}$ & 0.44 \\
\hline
\end{tabular}

Legend: IRFDsub: Submaximal Isometric Rate of Force Development values; ${ }^{\circ}$ : significant difference $p<0.05,{ }^{*}$ : significant difference after Bonferroni adjustment $p<0.0125$; ES: effect size $(\eta 2=.01$; small effect, $\eta 2=.06$; moderate effect, $\eta 2=$ .14 ; large effect)

\section{Additional file 10}

Outcome values of IRFDsub 0-30ms (N/ms) data and between group comparison at BASE, 4 $\mathrm{W}$ and $8 \mathrm{~W}$

\begin{tabular}{lllllll}
\hline & BASE & $\mathbf{p} / \mathbf{n}^{2}$ & $\mathbf{4 W}$ & $\mathbf{p} / \mathbf{n}^{2}$ & $\mathbf{8 W}$ & $\mathbf{p} / \mathbf{n}^{2}$ \\
\hline IRFDsub 0-30ms right ex (N/ms) (IG) & $0.32 \pm 0.2$ & $0.70 / 0.006$ & $0.44 \pm 0.2$ & $0.14 / 0.10$ & $0.63 \pm 0.3$ & $0.016^{*} / 0.21$ \\
IRFDsub 0-30ms right ex (N/ms) (SG) & $0.29 \pm 0.2$ & & $0.30 \pm 0.2$ & & $0.38 \pm 0.2$ & \\
IRFDsub 0-30ms left ex (N/ms) (IG) & $0.26 \pm 0.2$ & $0.18 / 0.07$ & $0.44 \pm 0.3$ & $0.10 / 0.11$ & $0.61 \pm 0.3$ & $0.001^{*} / 0.36$ \\
IRFDsub 0-30ms left ex (N/ms) (SG) & $0.34 \pm 0.3$ & & $0.29 \pm 0.2$ & & $0.33 \pm 0.2$ & $<.0 .001^{*} / 0.58$ \\
IRFDsub 0-30ms right flex (N/ms) (IG) & $0.10 \pm 0.05$ & $0.67 / 0.007$ & $0.24 \pm 0.1$ & $0.002^{*} / 0.32$ & $0.46 \pm 0.2$ & $<$ \\
IRFDsub 0-30ms right flex (N/ms) (SG) & $0.12 \pm 0.1$ & & $0.11 \pm 0.05$ & & $0.11 \pm 0.04$ & \\
IRFDsub 0-30ms left flex (N/ms) (IG) & $0.12 \pm 0.1$ & 0.309 & $0.24 \pm 0.1$ & $0.004^{*} / 0.28$ & $0.41 \pm 0.2$ & $<0.001^{*} / 0.56$ \\
IRFDsub 0-30ms left flex (N/ms) (SG) & $0.09 \pm 0.05$ & & $0.11 \pm 0.07$ & & $0.11 \pm 0.09$ &
\end{tabular}

Legend: IRFDsub: Submaximal Isometric Rate of Force Development values, N: Newton; IG: intervention group, SG: sham group, p: between groups, ex: extension, flex: flexion, ms: milisecond, ${ }^{\circ}$ : significant difference $p<0.05,{ }^{*}$ : significant difference after Bonferroni correction $p<0.025, \eta^{2}$ : effect size: $\eta^{2}=.01$; small effect, $\eta^{2}=.06$; moderate effect, $\eta^{2}=.14$; large effect 


\section{Additional file 11}

ANOVA with repeated measurements (ranks) intergroup-by-time effects and group-by-time interaction for the secondary outcomes IRFDsub 0-50ms (N/ms)

\begin{tabular}{lllll}
\hline & $\begin{array}{l}\text { Pillai s trace } \\
\left(\mathbf{r}^{2}=\mathbf{S S}_{\mathbf{B e t}} / \mathbf{S S}_{\text {Tot }}\right)\end{array}$ & $\begin{array}{l}\mathbf{L} \\
{\left[(\mathbf{N}-\mathbf{1}) \mathbf{r}^{2}\right]}\end{array}$ & $\mathbf{p ~}$ & ES $\left(\mathbf{n}^{2}\right)$ \\
\hline IRFDsub 0-50ms right ex (N/ms) (time effects) & 0.001 & 0.005 & 1.00 & 0.001 \\
IRFDsub 0-50ms right ex (N/ms) (interaction effects) & 0.26 & 4.92 & $0.02^{*}$ & 0.26 \\
IRFDsub 0-50ms left ex (N/ms) (time effects) & 0.001 & 0.14 & 0.99 & 0.001 \\
IRFDsub 0-50ms left ex (N/ms) (interaction effects) & 0.45 & 13.84 & $>0.001^{*}$ & 0.50 \\
IRFDsub 0-50ms right flex (N/ms) (time effects) & 0.001 & 0.01 & 0.99 & 0.001 \\
IRFDsub 0-50ms right flex (N/ms) (interaction effects) & 0.46 & 12.02 & $>0.001^{*}$ & 0.46 \\
IRFDsub 0-50ms left flex (N/ms) (time effects) & 0.001 & 0.003 & 1.00 & 0.001 \\
IRFDsub 0-50ms left flex (N/ms) (interaction effects) & 0.19 & 3.31 & $0.05^{\circ}$ & 0.19 \\
\hline
\end{tabular}

Legend: IRFDsub: Submaximal Isometric Rate of Force Development values; ${ }^{\circ}$ : significant difference $p<0.05$, *: significant difference after Bonferroni adjustment $\mathrm{p}<0.0125$; ES: effect size $(\eta 2=.01$; small effect, $\eta 2=.06$; moderate effect, $\eta 2=$ .14; large effect

\section{Additional file 12}

Outcome values af IRFDsub 0-50ms ( $\mathrm{N} / \mathrm{ms})$ data and between group comparison at BASE, 4 $\mathrm{W}$ and $8 \mathrm{~W}$

\begin{tabular}{lllllll}
\hline & BASE & $\mathbf{p} / \mathbf{\eta}^{2}$ & $\mathbf{4 W}$ & $\mathbf{p} / \mathbf{n}^{2}$ & $\mathbf{8 W}$ & $\mathbf{p} / \mathbf{\eta}^{2}$ \\
\hline RFDsub 0-50ms right ex (N/ms) (IG) & $0.34 \pm 0.2$ & $0.77 / 0.003$ & $0.63 \pm 0.3$ & $<0.001 / 0.003$ & $0.56 \pm 0.2$ & $0.003^{*} / 0.27$ \\
IRFDsub 0-50ms right ex (N/ms) (SG) & $0.29 \pm 0.2$ & & $0.36 \pm 0.3$ & & $0.25 \pm 0.1$ & \\
IRFDsub 0-50ms left ex (N/ms) (IG) & $0.31 \pm 0.3$ & $0.24 / 0.05$ & $0.53 \pm 0.3$ & $0.003^{*} / 0.27$ & $0.75 \pm 0.4$ & $0.001^{*} / 0.33$ \\
IRFDsub 0-50ms left ex (N/ms) (SG) & $0.32 \pm 0.1$ & & $0.25 \pm 0.2$ & & $0.30 \pm 0.2$ & $<0.001^{*} / 0.59$ \\
IRFDsub 0-50ms right flex (N/ms) (IG) & $0.10 \pm 0.05$ & $0.44 / 0.02$ & $0.6 \pm 0.1$ & $<0.001^{*} / 0.43$ & $0.39 \pm 2$ & \\
IRFDsub 0-50ms right flex (N/ms) (SG) & $0.12 \pm 0.07$ & & $0.10 \pm 0.04$ & & $0.11 \pm 0.05$ & \\
IRFDsub 0-50ms left flex (N/ms) (IG) & $0.13 \pm 0.1$ & $0.67 / 0.006$ & $0.29 \pm 0.2$ & $0.003^{*} / 0.27$ & $0.42 \pm 0.2$ & $<0.001^{*} / 0.41$ \\
IRFDsub 0-50ms left flex (N/ms) (SG) & $0.09 \pm 0.05$ & & $0.10 \pm 0.06$ & & $0.10 \pm 0.06$ & \\
\hline
\end{tabular}

Legend: IRFDsub: Submaximal Isometric Rate of Force Development values, N: Newton; IG: intervention group, SG: sham group, p: between groups, ex: extension, flex: flexion, ms: milisecond, ${ }^{\circ}$ : significant difference $p<0.05$, *: significant difference after Bonferroni correction $p<0.025, \eta^{2}$ : effect size: $\eta^{2}=.01$; small effect, $\eta^{2}=.06$; moderate effect, $\eta^{2}=.14$; large effect 


\section{Additional file 13}

ANOVA with repeated measurements (ranks) intergroup-by-time effects and group-by-time interaction for the secondary outcomes IRFDsub 0-100ms (N/ms)

\begin{tabular}{|c|c|c|c|c|}
\hline & $\begin{array}{l}\text { Pillai s trace } \\
\left(\mathrm{r}^{2}=\mathrm{SS}_{\mathrm{Bet}} / \mathrm{SS}_{\mathrm{Tot}}\right)\end{array}$ & $\begin{array}{l}\mathrm{L} \\
{\left[(\mathrm{N}-1) \mathrm{r}^{2}\right]}\end{array}$ & $p$ & ES $\left(\eta^{2}\right)$ \\
\hline IRFDsub 0-100ms right ex (N/ms) (time effects) & 0.001 & 0.002 & 1.00 & 0.001 \\
\hline IRFDsub 0-100ms right ex (N/ms) (interaction effects) & 0.11 & 1.65 & 0.211 & 0.11 \\
\hline IRFDsub 0-100ms left ex (N/ms) (time effects) & 0.001 & 1.12 & 0.99 & 0.001 \\
\hline IRFDsub 0-100ms left ex (N/ms) (interaction effects) & 0.30 & 6.09 & $0.008^{*}$ & 0.30 \\
\hline IRFDsub 0-100ms right flex (N/ms) (time effects) & 0.001 & 0.011 & 0.99 & 0.001 \\
\hline IRFDsub 0-100ms right flex (N/ms) (interaction effects) & 0.429 & 10.54 & $>0.001^{*}$ & 0.43 \\
\hline IRFDsub 0-100ms left flex (N/ms) (time effects) & 0.001 & 0.007 & 0.99 & 0.001 \\
\hline IRFDsub 0-100ms left flex (N/ms) (interaction effects) & 0.32 & 6.61 & $0.004^{*}$ & 0.32 \\
\hline
\end{tabular}

Legend: IRFDsub: Submaximal Isometric Rate of Force Development values; ${ }^{\circ}$ : significant difference $p<0.05,{ }^{*}$ : significant difference after Bonferroni adjustment $\mathrm{p}<0.0125$; ES: effect size $(\eta 2=.01$; small effect, $\eta 2=.06$; moderate effect, $\eta 2=$ 14; large effect

\section{Additional file 14}

Outcome values af RFDsub $0-100 \mathrm{~ms}(\mathrm{~N} / \mathrm{ms})$ data and between group comparison at BASE, 4 $\mathrm{W}$ and $8 \mathrm{~W}$

\begin{tabular}{|c|c|c|c|c|c|c|}
\hline & BASE & $\mathrm{p} / \mathbf{n}^{2}$ & $4 W$ & $\mathrm{p} / \mathbf{n}^{2}$ & $8 W$ & $\mathrm{p} / \mathbf{n}^{2}$ \\
\hline IRFDsub 0-100ms right ex (N/ms) (IG) & $0.34 \pm 0.2$ & $0.42 / 0.02$ & $0.42 \pm 0.3$ & $0.13 / 0.12$ & $0.55 \pm 0.3$ & $0.006^{*} / 0.23$ \\
\hline IRFDsub 0-100ms right ex (N/ms) (SG) & $0.27 \pm 0.2$ & & $0.27 \pm 0.1$ & & $0.29 \pm 0.2$ & \\
\hline IRFDsub 0-100ms left ex (N/ms) (IG) & $0.34 \pm 0.4$ & $0.64 / 0.01$ & $0.50 \pm 0.4$ & $0.12 / 0.06$ & $0.71 \pm 0.4$ & $0.007 * / 0.23$ \\
\hline IRFDsub 0-100ms left ex (N/ms) (SG) & $0.31 \pm 0.2$ & & $0.32 \pm 0.2$ & & $0.31 \pm 0.2$ & \\
\hline IRFDsub 0-100ms right flex (N/ms) (IG) & $0.10 \pm 0.05$ & $0.43 / 0.02$ & $0.32 \pm 0.2$ & $<0.001^{*} / 0.44$ & $0.47 \pm 0.2$ & $<0.001^{*} / 0.55$ \\
\hline IRFDsub 0-100ms right flex (N/ms) (SG) & $0.12 \pm 0.08$ & & $0.11 \pm 0.05$ & & $0.12 \pm 0.05$ & \\
\hline IRFDsub 0-100ms left flex (N/ms) (IG) & $0.12 \pm 0.1$ & $0.97 / 0.01$ & $0.30 \pm 0.2$ & $<0.001^{*} / 0.40$ & $0.51 \pm 0.2$ & $<0.001 * / 0.56$ \\
\hline IRFDsub 0-100ms left flex (N/ms) (SG) & $0.09 \pm 0.05$ & & $0.09 \pm 0.05$ & & $0.10 \pm 0.07$ & \\
\hline
\end{tabular}

Legend: IRFDsub: Submaximal Isometric Rate of Force Development values, N: Newton; IG: intervention group, SG: sham group, p: between groups, ex: extension, flex: flexion, ms: milisecond, ${ }^{\circ}$ : significant difference $p<0.05$, $^{*}$ : significant difference after Bonferroni correction $p<0.025, \eta^{2}$ : effect size: $\eta^{2}=.01$; small effect, $\eta^{2}=.06$; moderate effect, $\eta^{2}=.14$; large effect 


\section{Additional file 15}

ANOVA with repeated measurements (ranks) intergroup-by-time effects and group-by-time interaction for the secondary outcomes IRFDsub 100-200ms (N/ms)

\begin{tabular}{|c|c|c|c|c|}
\hline & $\begin{array}{l}\text { Pillai`s trace } \\
\left(r^{2}=S_{B_{\mathrm{Bet}}} / \mathrm{SS}_{\mathrm{Tot}}\right)\end{array}$ & $\begin{array}{l}\mathrm{L} \\
{\left[(\mathrm{N}-1) \mathrm{r}^{2}\right]}\end{array}$ & $\mathbf{p}$ & ES $\left(\eta^{2}\right)$ \\
\hline IRFDsub 100-200ms right ex (N/ms) (time effects) & 0.01 & 0.15 & 0.87 & 0.01 \\
\hline IRFDsub 100-200ms right ex (N/ms) (interaction effects) & 0.29 & 5.26 & $0.01 *$ & 0.29 \\
\hline IRFDsub 100-200ms left ex (N/ms) (time effects) & 0.02 & 0.22 & 0.80 & 0.02 \\
\hline IRFDsub 100-200ms left ex (N/ms) (interaction effects) & 0.37 & 7.60 & $0.003^{*}$ & 0.37 \\
\hline IRFDsub 100-200ms right flex (N/ms) (time effects) & 0.003 & 0.04 & 0.96 & 0.003 \\
\hline IRFDsub 100-200ms right flex (N/ms) (interaction effects) & 0.43 & 9.70 & $0.001^{*}$ & 0.43 \\
\hline IRFDsub 100-200ms left flex (N/ms) (time effects) & 0.02 & 0.26 & 0.77 & 0.02 \\
\hline IRFDsub 100-200ms left flex (N/ms) (interaction effects) & 0.32 & 6.10 & $0.007^{*}$ & 0.38 \\
\hline
\end{tabular}

Legend: IRFDsub: Submaximal Isometric Rate of Force Development values; ${ }^{\circ}$ : significant difference $p<0.05,{ }^{*}$ : significant difference after Bonferroni adjustment $p<0.0125$; ES: effect size $(2=.01$; small effect, $2=.06$; moderate effect, $2=$ .14; large effect

\section{Additional file 16}

Outcome values af RFDsub 100-200ms ( $\mathrm{N} / \mathrm{ms})$ data and between group comparison at BASE, $4 \mathrm{~W}$ and $8 \mathrm{~W}$

\begin{tabular}{lllllll}
\hline & BASE & $\mathbf{p ~ / \mathbf { n } ^ { 2 }}$ & $\mathbf{4 W}$ & $\mathbf{p ~ / \mathbf { n } ^ { 2 }}$ & $\mathbf{8 W}$ & $\mathbf{p} / \mathbf{\eta}^{2}$ \\
\hline IRFDsub 100-200ms right ex (N/ms) (IG) & $0.28 \pm 0.2$ & $0.92 / 0.01$ & $0.39 \pm 0.2$ & $0.005^{\star} / 0.26$ & $0.56 \pm 0.3$ & $<0.003^{*} / 0.30$ \\
IRFDsub 100-200ms right ex (N/ms) (SG) & $0.27 \pm 0.1$ & & $0.21 \pm 0.1$ & & $0.28 \pm 0.1$ & \\
IRFDsub 100-200ms left ex (N/ms) (IG) & $0.27 \pm 0.3$ & $0.94 / 0.01$ & $0.45 \pm 0.3$ & $0.003^{*} / 0.29$ & $0.65 \pm 0.2$ & $<0.001^{*} / 0.61$ \\
IRFDsub 100-200ms left ex (N/ms) (SG) & $0.21 \pm 0.1$ & & $0.21 \pm 0.1$ & & $0.27 \pm 0.1$ & \\
IRFDsub 100-200ms right flex (N/ms) (IG) & $0.08 \pm 0.04$ & $0.32 / 0.04$ & $0.32 \pm 0.2$ & $<0.001^{*} / 0.50$ & $0.46 \pm 0.2$ & $<0.001^{*} / 0.54$ \\
IRFDsub 100-200ms right flex (N/ms) (SG) & $0.10 \pm 0.08$ & & $0.11 \pm 0.1$ & & $0.11 \pm 0.1$ & \\
IRFDsub 100-200ms left flex (N/ms) (IG) & $0.17 \pm 0.2$ & $0.77 / 0.03$ & $0.39 \pm 0.2$ & $<0.001^{*} / 0.48$ & $0.60 \pm 0.2$ & $<0.001^{*} / 0.70$ \\
IRFDsub 100-200ms left flex (N/ms) (IG) & $0.10 \pm 0.08$ & & $0.08 \pm 0.6$ & & $0.09 \pm 0.06$ & \\
\hline
\end{tabular}

Legend: IRFDsub: Submaximal Isometric Rate of Force Development values, N: Newton; IG: intervention group, SG: sham group, p: between groups, ex: extension, flex: flexion, ms: milisecond, ${ }^{\circ}$ : significant difference $p<0.05$, $^{*}$ : significant difference after Bonferroni correction $p<0.025, \eta^{2}$ : effect size: $\eta^{2}=.01$; small effect, $\eta^{2}=.06$; moderate effect, $\eta^{2}=.14$; large effec 



\title{
Chapter 8
}

\author{
Sensor-based foot-mounted wearable system \\ and pressure sensitive gait analysis \\ Agreement in frail older adults in long-term care
}




\section{ABSTRACT}

Hintergrund: Das RehaWatch ${ }^{\circledR}$ System ist ein tragbares Beschleunigungsmessgerät zur Messung von Gangparametern, die eine gute Validität bei jungen Erwachsenen zeigt. Allerdings fehlen Daten zur Validität von älteren Menschen in Langzeitpflege. Ziel: Das Ziel dieser Studie war es, die Validität des RehaWatch ${ }^{\circledR}$ System mit dem GAITRite ${ }^{\circledR}$ System bei älteren Menschen in Langzeitpflege zu vergleichen. Material und Methoden: 23 ältere Probanden (Durchschnittsalter: $90.9 \pm$ 8,4 Jahre) führten bei normaler und hoher Laufgeschwindigkeit Einfach- und Doppelaufgaben aus. Dabei wurden gleichzeitig vom RehaWatch ${ }^{\circledR}$ system und dem GAITRite ${ }^{\circledR}$ system Daten gesammelt um mittels Bland-AltmanPlots die Übereinstimmungsgrenzen festzustellen. Ergebnisse Der Mean-Bias für Schrittdauer, Laufgeschwindigkeit und Trittfrequenz lag über die a-priori festgelegte $\pm 7 \%$ Grenze für normales Gehen mit Einfach- und Doppelaufgaben. Die Übereinstimmungsgrenzen lagen zwischen -21\% und $15 \%$. Nur die Kadenz zeigte eine schmale Übereinstimmungsgrenze für Einfachaufgaben $(-4.1 \%$ bis $3 \%)$ und Doppelaufgaben $(-8.4 \%$ bis $7.7 \%)$. Heterogene Bias wurden für Schrittdauer beim schnellen Gehen für Doppelaufgaben und für Geschwindigkeit beim schnellen Gehen für Einfach- und Doppelaufgaben bestimmt. Heteroskedastizität wurde für Schrittlänge während normalen Gehen mit Doppelaufgaben und schnellem Gehen für Einfachaufgaben ermittelt. Schlussfolgerung: Es kann zwischen den beiden Systemen zum normalen Gehen mit Einfachund Doppelaufgaben kein Gangparameter genutzt werden.

Schlüsselwörter: Datengenauigkeit, Gang, Gebrechlichkeit, 


\section{ABSTRACT}

\section{Background:}

the RehaWatch ${ }^{\circledast}$ system is a portable accelerometer for measurement of gait parameters that shows good validity in young adults. However, validity data are missing for long-term-care (LTC) elderly. Aim: The aim was to evaluate the concurrent validity of the RehaWatch ${ }^{\circledR}$ system using the GAITRite ${ }^{\circledR}$ system as a criterion reference for gait assessment in LTC elderly.

\section{Material and Methods:}

23 elderly participants (mean age: $90.9 \pm 8.4$ years) performed four walking trials at normal and fast walking speed during single-task and dual-task walking. Data were collected simultaneously for each trial from both systems. Concurrent validity was assessed through limits of agreement (LoA) methodology using Bland-Altman plots.

\section{Results:}

no systematic bias could be determined. Mean biases for step duration, velocity and cadence were above the pre-specified $\pm 7 \%$ value from zero lines for normal walking during single-task and dual-task walking. LoA lay in a wide range between -21\% and 25\%. Only cadence showed small LoA for normal walking speed during single- $(-8.4 \%$ to $7.7 \%)$ and dual-tasking $(-4.1 \%$ to $3 \%)$. Heterogeneous bias was determined for step duration during fast walking during dual-task and for velocity during fast walking during single-task and dual-task. Heteroscedasticity was shown for step length during normal walking under the dual-task condition and fast walking during single-task and dual-task.

\section{Summary}

No gait parameters are interchangeable usable between the two systems for normal walking during single-task and dual-task.

\section{Keywords:}

data accuracy, frail elderly, gait 


\section{Introduction}

Frailty is characterised by reduced reserve capacities of multiple organ systems and increased vulnerability to internal and external stressors [1]. Gait impairments in form of lowered velocity and changes in spatial-temporal variables are related to the frailty status of older adults [2,3]. For clinical and research settings, therefore, objective measurements of spatial-temporal gait parameters are relevant to identify possible gait impairments [4-7] or to evaluate the effects of therapeutic interventions in frail populations. However, a recent systematic review concluded that so far gait characteristics in frail older persons are rather insufficiently analysed [8].

During walking, the environment has to be observed and changes such as jerking to a stop at a red light will immediately occur with balance reactions [9]. For this dual-task condition, muscle strength, balance, and cognitive response are needed [10]. Elderly individuals are often unable to automatically adapt their gait to the environment due to limited resources, often caused by degenerative processes such as desensitization of muscle spindles, reduction of sensory, motor and inhibitory nerve cells, and muscle atrophy [10, 11]. Walking and cognition deficits have been shown to predict falls [12]. Therefore, evaluation of walking while stressing the cognitive capacity; e.g. through performing a dual-task (DT), is of scientific interest in frail older adults [8].

Different valid and reliable methods exist for the assessment of gait parameters, e.g. force plates, three-dimensional motion analyses systems, pressure-activated sensors, and accelerometers $[6,7,13-15]$. However, most methods are either time-consuming, costly, limited to a single gait cycle, labour-intensive, or lack applicability in frail populations. As an example, the GAITRite ${ }^{\circledR}$ system is a reliable and valid portable electronic walkway for measuring both averaged and individual step parameters in older populations $[13,16]$, however, a drawback of the system is that multiple runs containing repetitive stop and go movements that potentially bias the measurements are required to obtain estimates of variability measures [3]. Furthermore, it is questionable whether data collected in a laboratory environment from individual steps adequately mimic natural walking performance in daily life [17]. Therefore, user-friendly portable gait analysis systems which are able to capture data from many gait cycles in frail older adults and that would allow measurements in a more challenging context are potentially important for clinical and research settings.

Recently, accelerometer-based gait analysis systems fixed at different body locations have been proposed for ambulatory gait assessment and have been found valid and reliable for the analysis of gait parameters in non-frail populations [18-26]. However, most body fixed accelerometer based systems are still in need of further development in (frail) older adult populations [27] and trunk attached accelerometer approaches that depend on movements in the direction of the vertical axis show limitations because of the inability of many frail individuals to perform stepping movements [28]. The use of foot mounted sensors, that have been used most commonly 
to assess spatio-temporal parameters of gait [29], can be hypothesised to be a promising opportunity for ambulatory gait monitoring in frail individuals. It is not clear, however, whether the application of this approach in frail elderly is valid.

The aim of this study was 1) to determine the concurrent validity of a shoe mounted wearable gait assessment system (RehaWatch ${ }$ ) with the GAITRite ${ }^{\circledR}$ system for spatial-temporal gait parameters that were recorded for averaged step data in a frail older adult population living in long term care and 2) to compare the levels of agreement (LOA) for averaged step data from different walking conditions; e.g. single and dual task walking while walking at preferred and fast speed.

\section{Purpose and Methods}

\section{Subjects}

Twenty-three LTC care dependent elderly (mean age: $90.9 \pm 8.4$ years; height: $1.60 \pm 0.1$ m; weight: $65.0 \pm 17.8 \mathrm{~kg}$ ) were recruited from a nursing home in Canton Bern, Switzerland, based on convenience sampling. Participants were recruited by means of an individual interview performed between November and December 2012 and were prospectively enrolled when they fulfilled the following inclusion criteria: over 80 years old, Short Physical Performance Battery (SPPB) score $<6$ points, able to walk 15 meters with or without aids, living in Canton Bern, and having a score $>16$ in the Mini-Mental State Examination (MMSE). Exclusion criteria were: visual disturbances, limb prostheses, acute joint diseases, acute thrombosis, acute fractures, acute infections, acute tissue damage, or acute surgical scars. This study conforms to the ethical requirements for human experimentation in accordance with the latest version of the Declaration of Helsinki. The local Ethics committee Bern approved the study (KEK-147/12 Bern) and all subjects participating in the study provided informed consent.

\section{Data acquisition and study design}

The GAITRite ${ }^{\circledR}$ was used as a reference standard test [15] and the system used for this study was $13 \mathrm{~m}$ long and $0.89 \mathrm{~m}$ wide, with an active sensor area $7.32 \mathrm{~m}$ long and $0.61 \mathrm{~m}$ wide. The sampling rate of the system is $80 \mathrm{~Hz}$. Spatial-temporal gait parameters were processed and stored using the application software. The system has been shown to be accurate at measuring individual footstep parameters such that individual step values were within $1.5 \mathrm{~cm}$ and $0.02 \mathrm{~s}$ on the majority (80-94\%) of occasions [13].

The RehaWatch ${ }^{\circledR}$ uses inertial sensors reliably [30] and enables the measurement of acceleration and angular velocity [31]. Via a placeholder, the inertial sensors are fixed at the lateral malleolus of both the left and right leg. Each inertial sensor contains three accelerometers and three gyroscopes which capture foot movements in six degrees of freedom (three translational, three rotational). The accelerometer has a measuring range of $\pm 4 \mathrm{~g}$-force and the gyroscope of \pm $700 \%$ (sampling rate: $512 \mathrm{~Hz}$ ). Measured values are digitized at a sampling rate of $500 \mathrm{~Hz}$. 
A mobile computing device (data logger) keeps track of accumulated data and can either be attached to a participant's waist belt or hand-held by the therapist.

This study used a cross-sectional study design and follows the STARD publication guideline [32-34].

\section{Test procedure}

During the measurements, the subjects walked on the walkway while wearing their own comfortable clothing and low-heeled habitual shoes. Before starting the measurement procedure, leg length was measured from the greater trochanter to the lateral malleolus. To measure steady state walking, the central $7.32 \mathrm{~m}$ active sensor area of the GAITRite ${ }^{\circledR}$ system was used as the test distance and the trial, therefore, began and ended at a marked tape point on the floor approximately two meters from the walkway.

Subjects were positioned at the starting point and were assessed during walking under four different conditions AAgner, 2015 \#61\}\{Agner, 2015 \#61\}:

1) The participant walked at a self-selected preferred gait speed (single-task) receiving the information: "do not speak and try not to stop walking during the measurement". The participant was briefed to "walk like you would, when taking a letter to the mailbox".

2) The participant walked at a self-selected preferred gait speed with an additional cognitive task (dual-task). The participant had to walk and count aloud backwards in steps of seven, five or three, depending on ability. The participants were briefed to "try to walk and count at the same time. Do not favor one task over the other but try to perform these tasks concurrently".

3) The participant walked at their fastest walking speed. The participant received the information: "Try to walk as fast as possible without starting to run. Do not speak, and try not to stop walking during the measurement".

4) The participant walked at their fastest walking speed with an additional cognitive task. The participant had to walk and count aloud backwards in steps of seven, five, or three, depending on ability. The participant was briefed to "Try to walk as fast as possible without starting to run. Try to walk and count at the same time. Do not favor one task over the other but try to perform these concurrently".

The following gait parameters were used from both the GAITRite ${ }^{\circledR}$ and RehaWatch ${ }^{\circledR}$ systems: temporal parameters; stance duration left leg and right leg (seconds: s), walking velocity (meters/ second: $\mathrm{m} / \mathrm{s}$ ) and cadence (number of steps / walking time); spatial parameters; step length left and right (centimeters: $\mathrm{cm}$ ). 


\section{Statistical Analyses}

Descriptive statistics (mean SD) were used to describe demographic characteristics.

Gait parameters were tested for normality using the Kolmogorov-Smirnov test. For the evaluation of the agreement between the measurement systems the limits of agreement (LoA) were used, where the dispersion of the differences of the individual measured value-pairs are considered [35]. In this study, an acceptable agreement between the devices was deemed for a mean bias of $\pm 7 \%$ at maximum. Homogeneous bias and homoscedasticity were further a-priori established parameters indicating acceptable agreement between the RehaWatch ${ }^{\circledR}$ and GAITRite ${ }^{\circledR}$.

Bland-Altman plots were generated to provide a visual representation of heteroscedasticity by plotting the individual subject difference between the two systems against the individual mean of the two systems [36]. The LOAs between RehaWatch ${ }^{\circledR}$ and GAITRite ${ }^{\circledR}$ were expressed as intraclass correlation coefficients (ICC) of the type $(2, k)$, associated $95 \%$ confidence intervals $(\mathrm{Cl})$ and as repeatability coefficients (RC). RC was calculated according to [36] as 1.96 times the SD of the differences between both systems. The difference between the two measurement systems was expected to be less than $7 \%$. In the Bland-Altman plots, systematic error is present if the mean is greater than or less than zero. Systematic error is considered to be significant if both repeatability coefficients are on the same side of zero [37]. In addition, a logarithmic and antilogarithmic transformation was conducted to reach a uniform variability of the measured values over the whole measurement range. Paired t-tests were used to determine systematic differences between the two systems. Statistical significance was set at $p<0.05$.

SPSS version 21 (SPSS Inc., Chicago, IL, USA) and Microsoft ${ }^{\circledR}$ Excel ${ }^{\circledR}$ version 14.3.9 for Mac (Microsoft Corporation, Redmond, WA, USA) were used for the statistical analysis. 


\section{Results}

All 23 participants performed all trials but data from two individuals were rejected for analysis because of too excessive shuffling which meant no gait parameters could be derived for these two participants with neither system. Finally, data from 21 participants (mean age: 89.8 years, height: $1.61 \pm 0.2 \mathrm{~m}$, weight: $63.9 \pm 15.0 \mathrm{~kg}$ ) were evaluated, in which each participant performed 48 individual walking trials. Altogether, 1008 trials were available for data analysis.

The data were not normally distributed and skewed both for the RehaWatch ${ }^{\circledR}$ and the GAITRite ${ }^{\circledR}$, however, in opposing directions. For that reason, a log and antilog transformation was carried out. The log-transformed data presented a normal distribution. Simultaneously, using the exponential function, a back log-transformation of the measured values was carried out. Table 1 summarizes the mean and SD back log-transformed values for each measurement system of the gait parameters during the four task conditions.

\section{Agreement measures}

Table 1 presents comparative data for the two systems for the averaged values across four walking trials. Regardless of significance level, the mean values of Rehawatch were higher than those of GaitRite for all studied variables except step length of the left leg during fast walking and Cadence during fast walking while counting, as shown in Table 1. There were significant differences between the two systems in the majority of the parameters for the averaged step data, implying that based on the mean of the paired differences and supported by the t-test results bias between the methods was apparent. The LOAs between the two systems for averaged step data was moderate for step length (ICCs between 0.45-0.84, RCs between 1.16-2.00) and low for step duration, cadence and low for step duration and walking speed (ICCs between 0.03-0.84 and RCs between 0.18-1.94). 


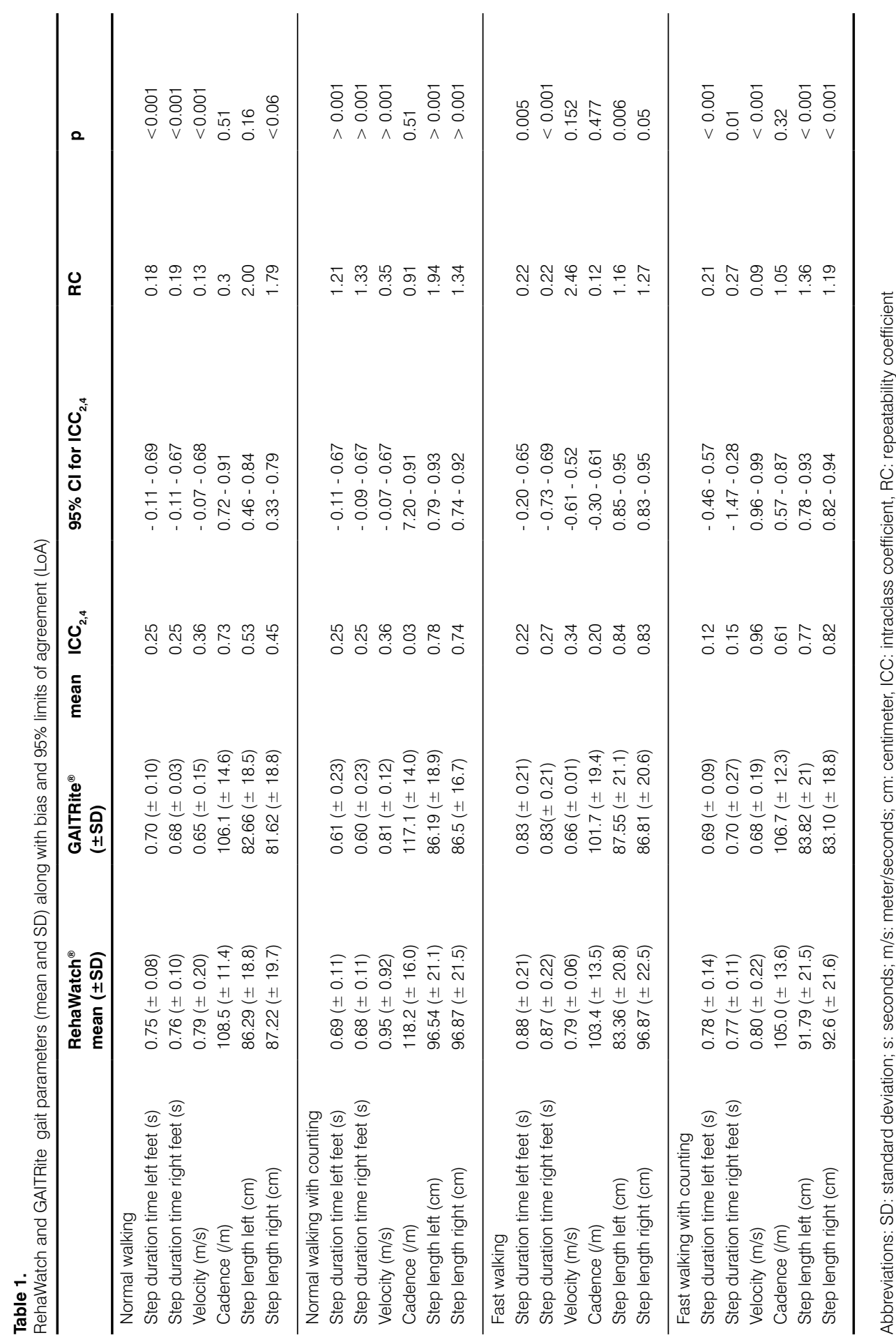




\section{Concurrent validity}

The Bland-Altman plot (Figure 2 - 5) shows log-transformed data.

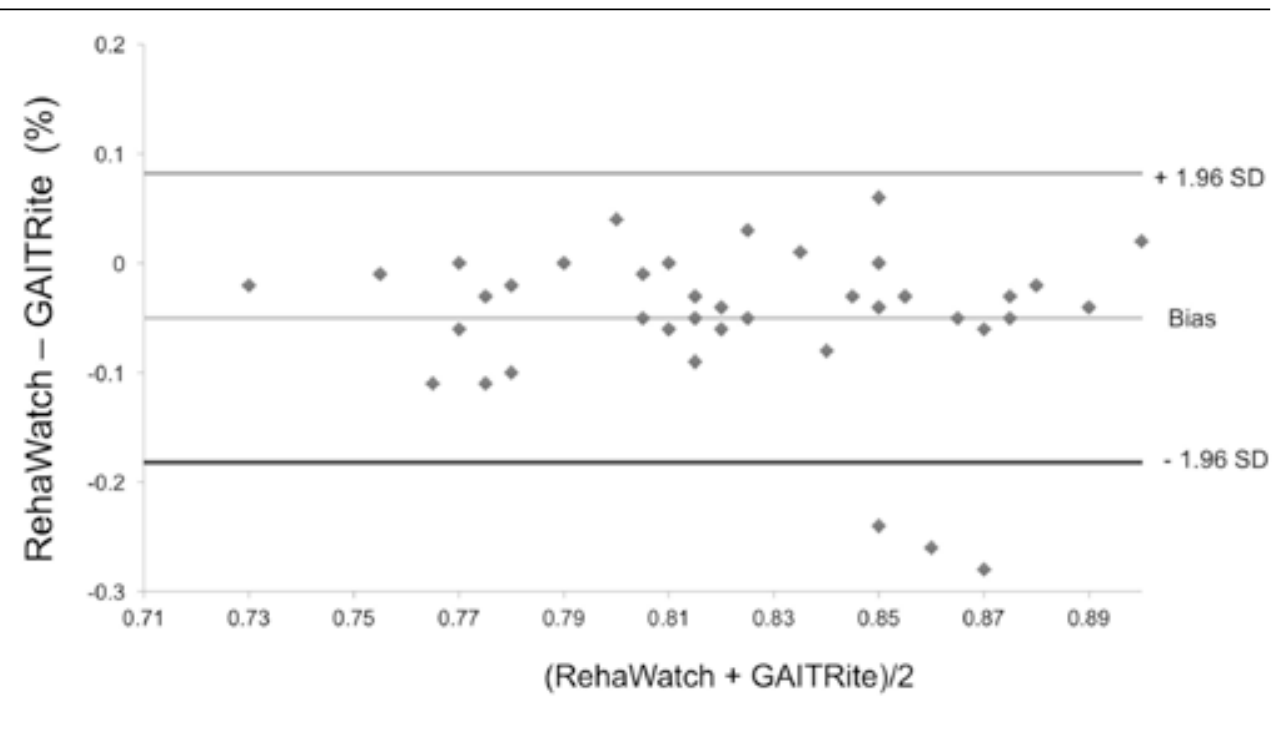

Fig. 2a: step duration time left leg [antilog) during normal walking during single-task

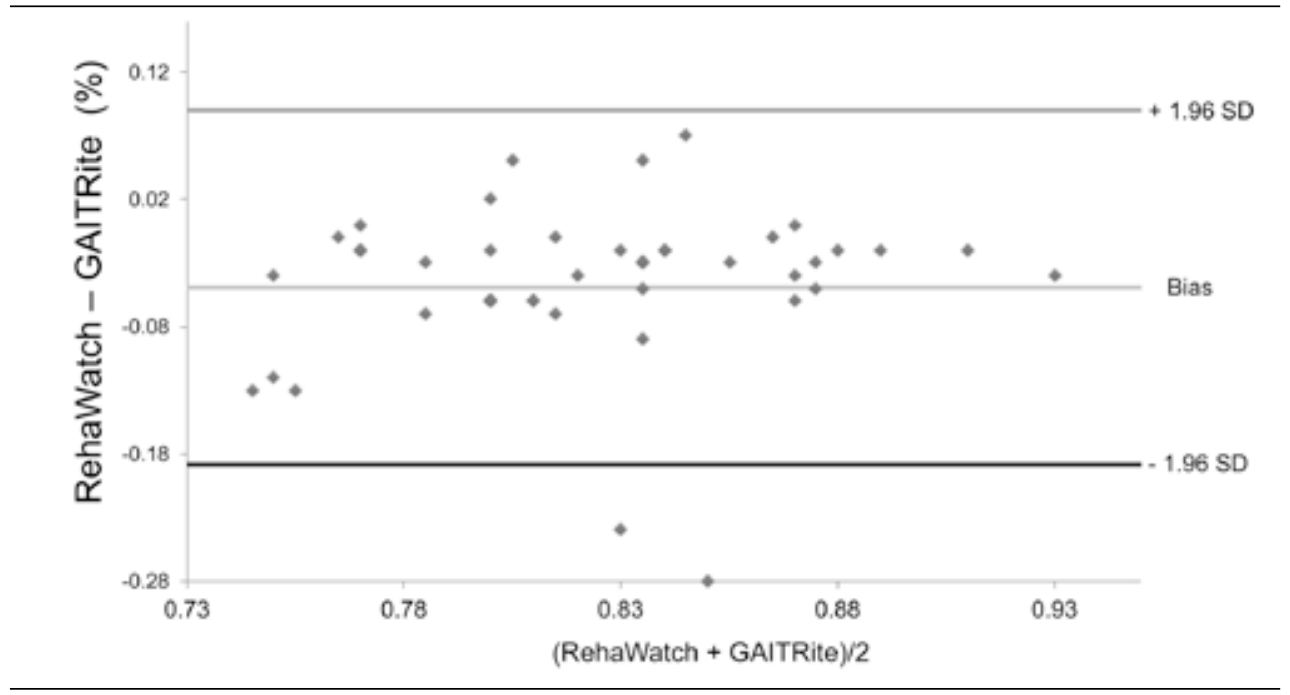

Fig 2b: step duration time rigth leg [log] during normal walking during single-task 


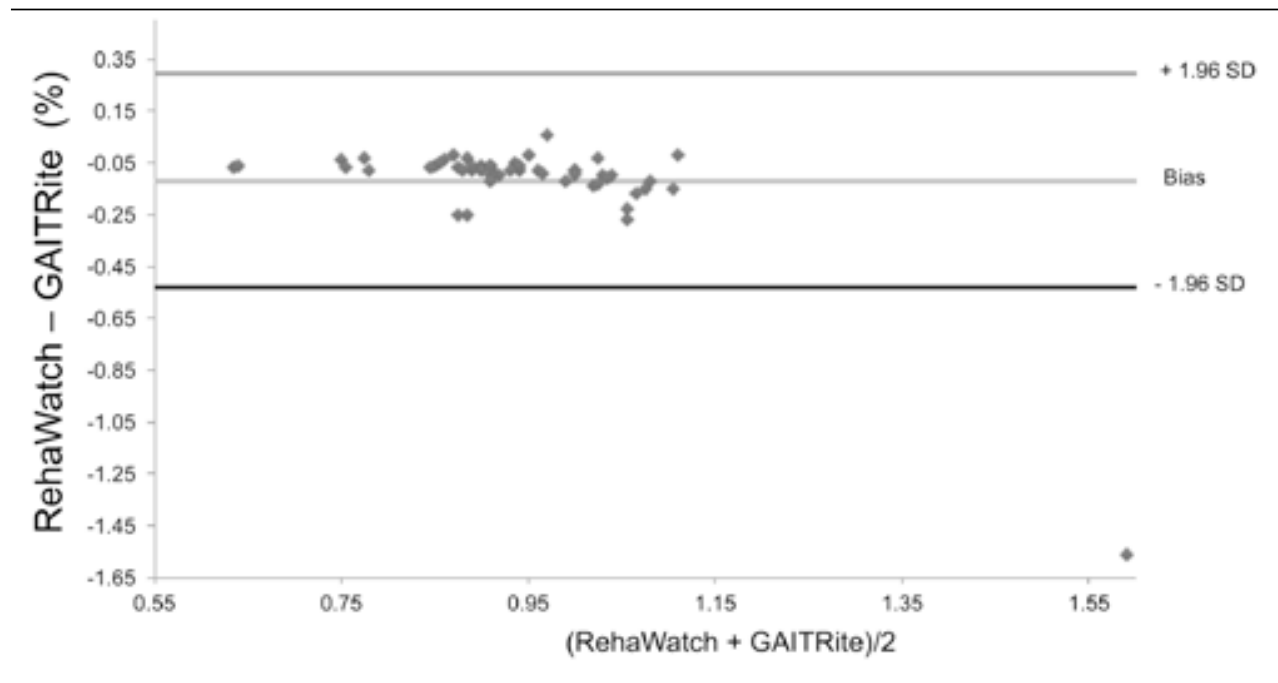

Fig 2c: velocity [antilog] during normal walking during single-task

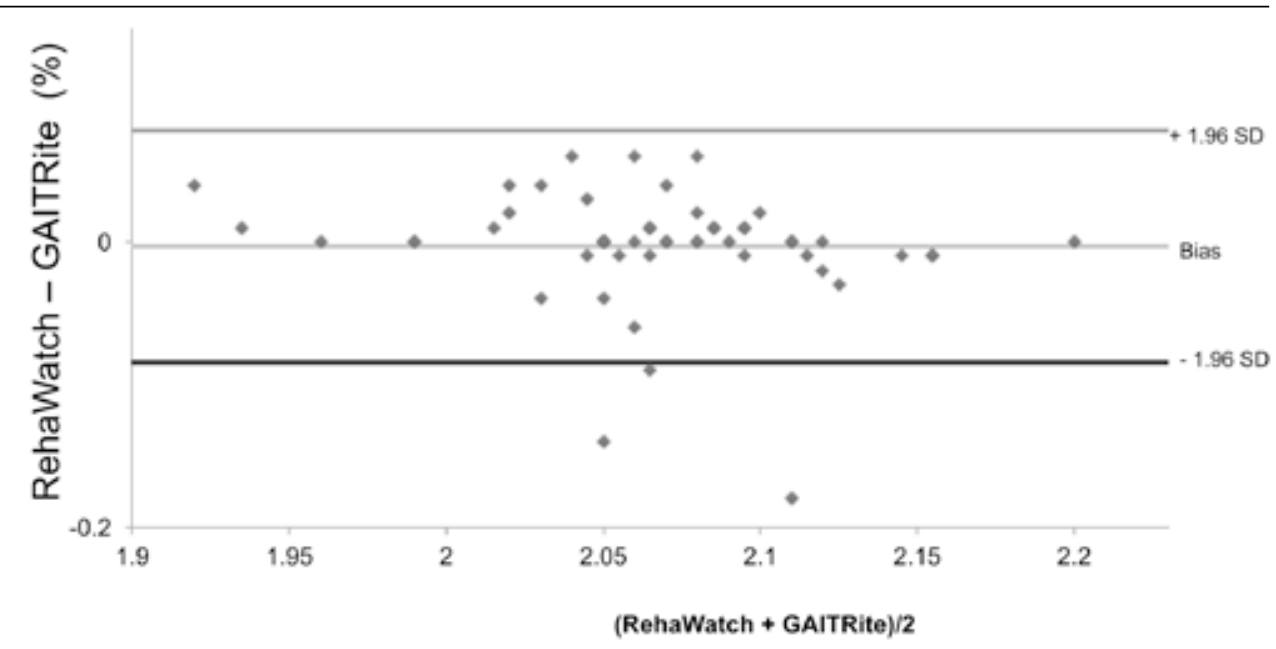

Fig. 2d: cadence [antilog] during normal walking during single-task 


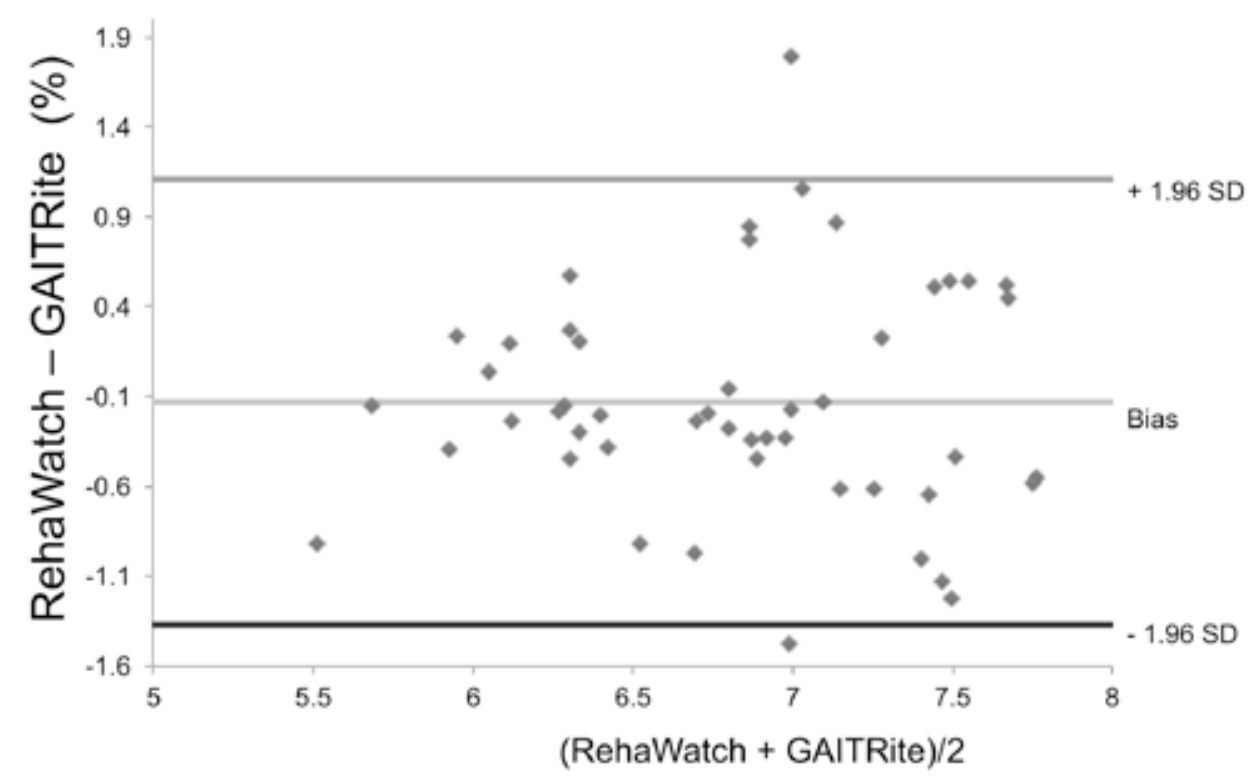

Fig. 2e: step length left leg [antilog] during normal walking during single-task

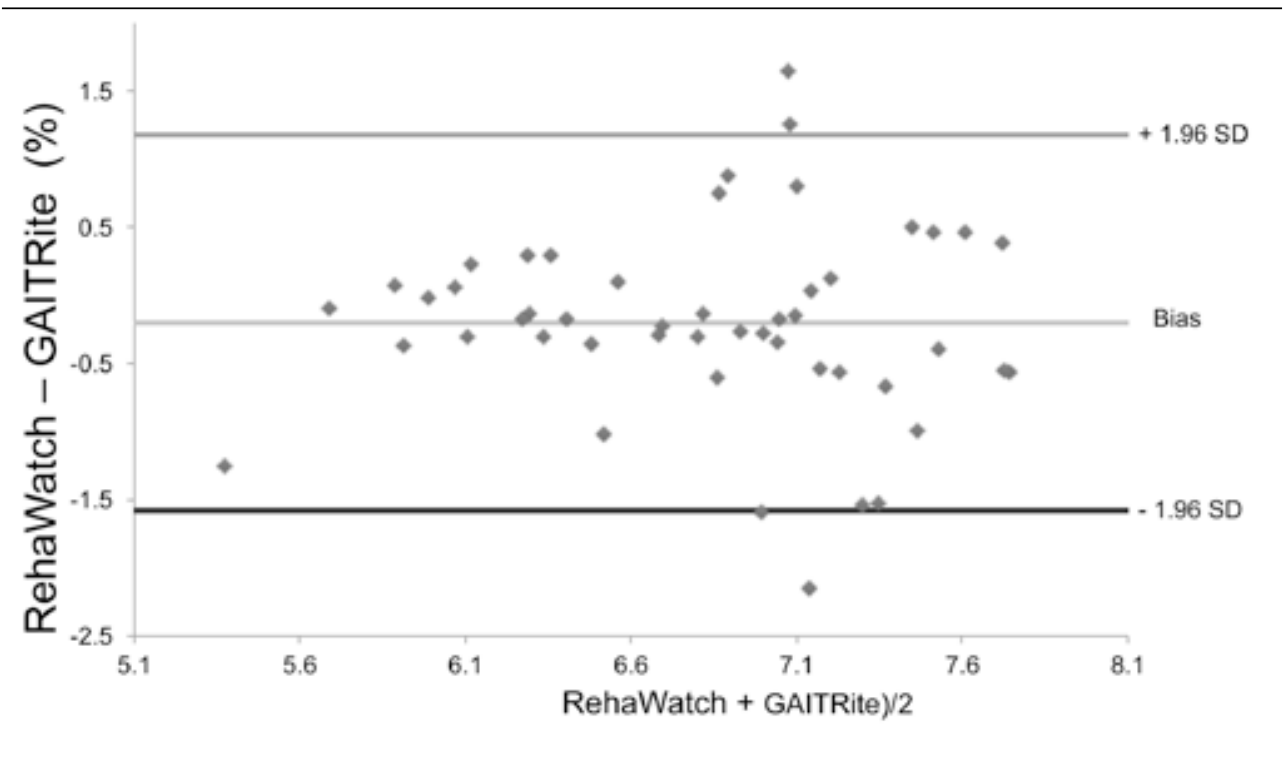

Fig. 2f: step lenght right leg [antlog] during normal walking during single-task 
Figure 3 and Figure 4 show negative and positive zero bias around the zero line for fast walking during single-task and dual-task. Systematic bias cannot be determined for temporal parameters and for spatial parameters.

However, heterogeneous bias for velocity is presented for normal walking during dual-task and fast walking during single-task and dual-task, and for step length left and right during fast walking during dual-task. Heteroscedasticity for step duration left and right could be determined in all attempts at walking.

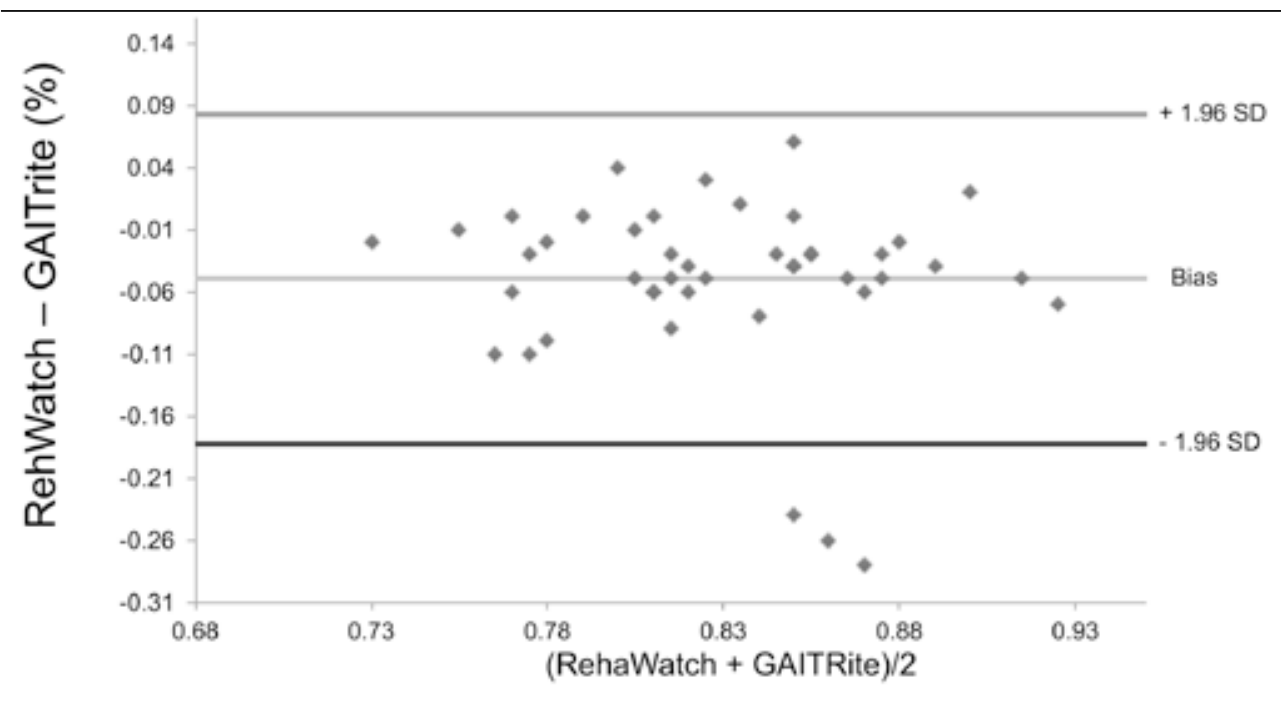

Fig. 3a: step duration time left leg [antlog] during normal walking during dual-task

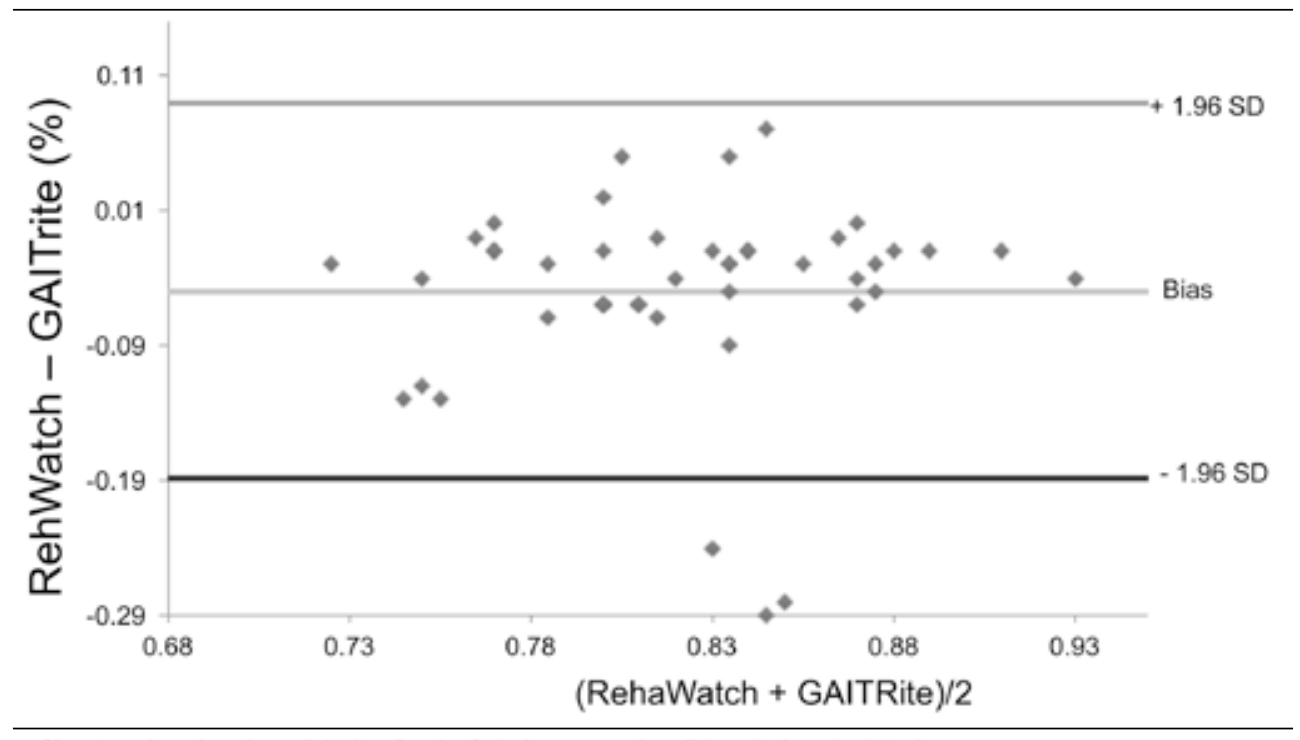

g. 3b: step duration time right leg [antlog] during normal walking during dual-task 


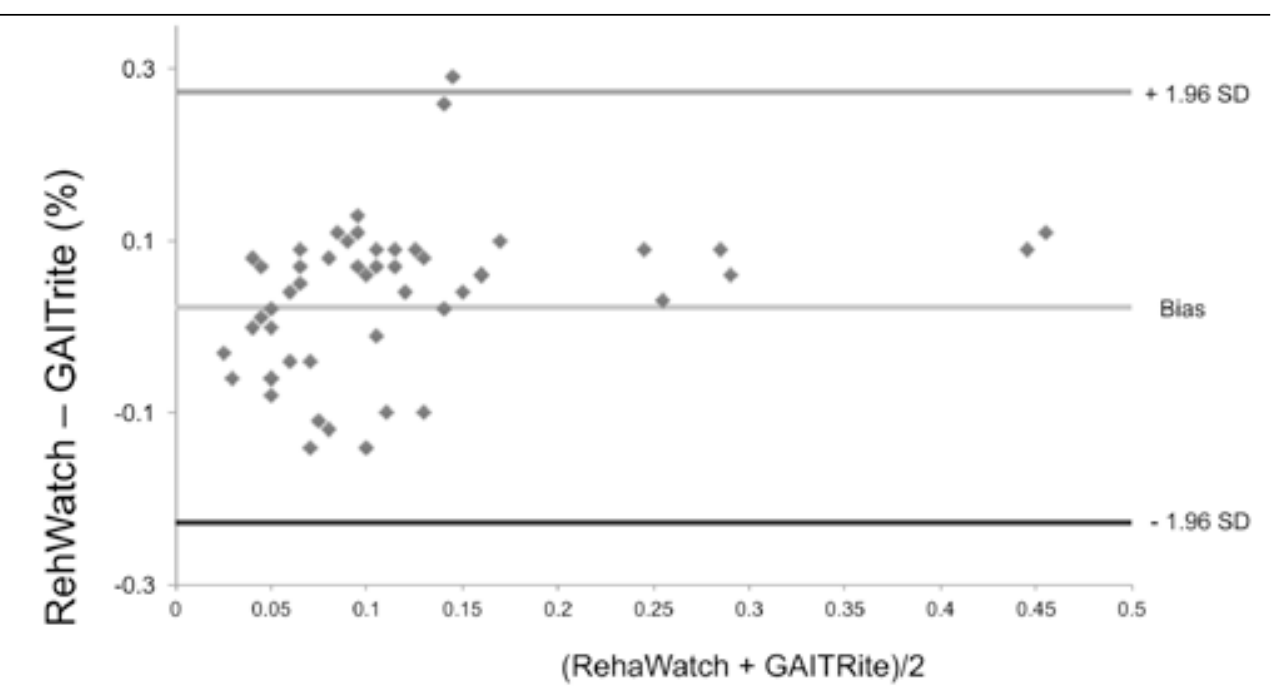

Fig. 3c: velocity [antlog] during during normal walking during and dual-task

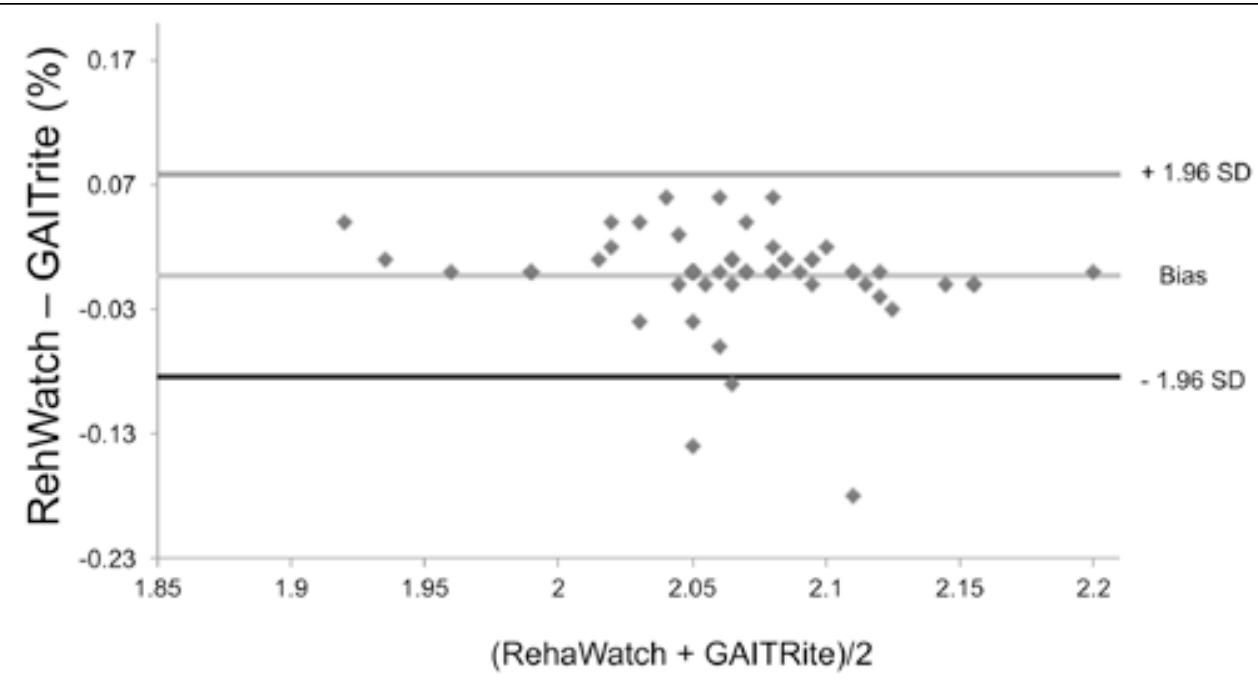

Fig. 3d: cadence [antilog] during normal walking during dual-task 


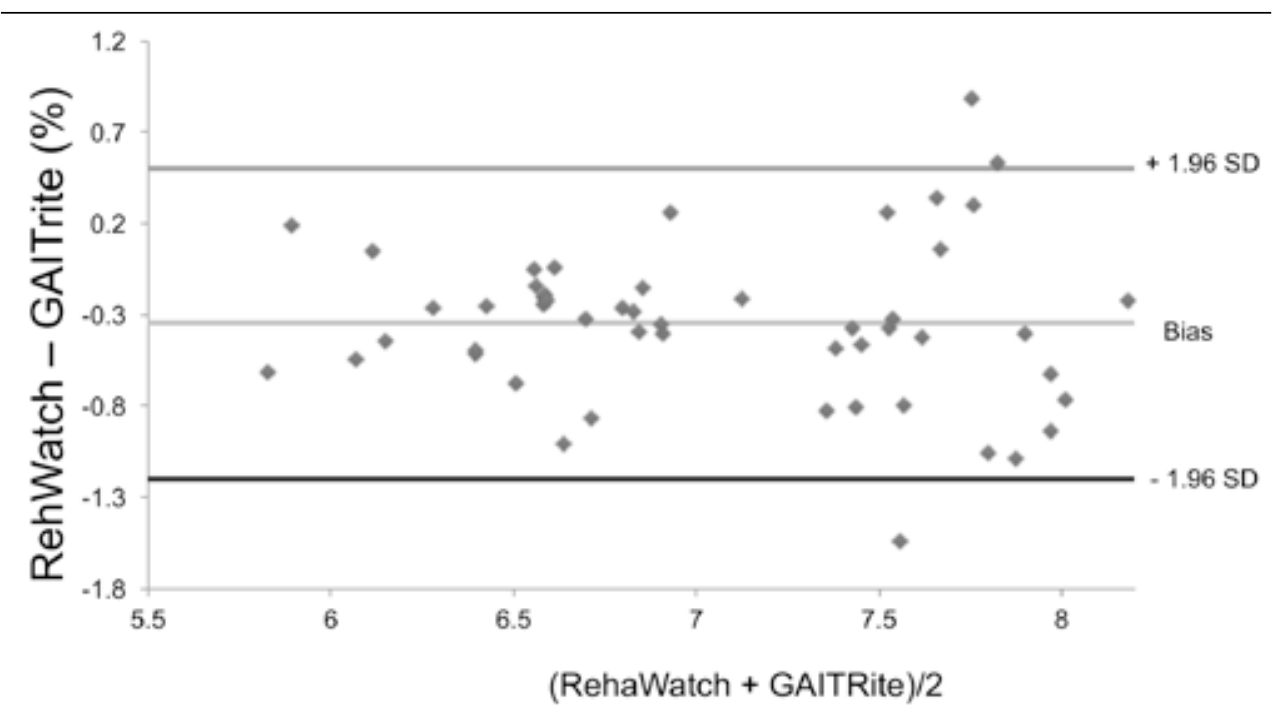

Fig. 3e: step length left leg [antilog] normal walking during dual-task

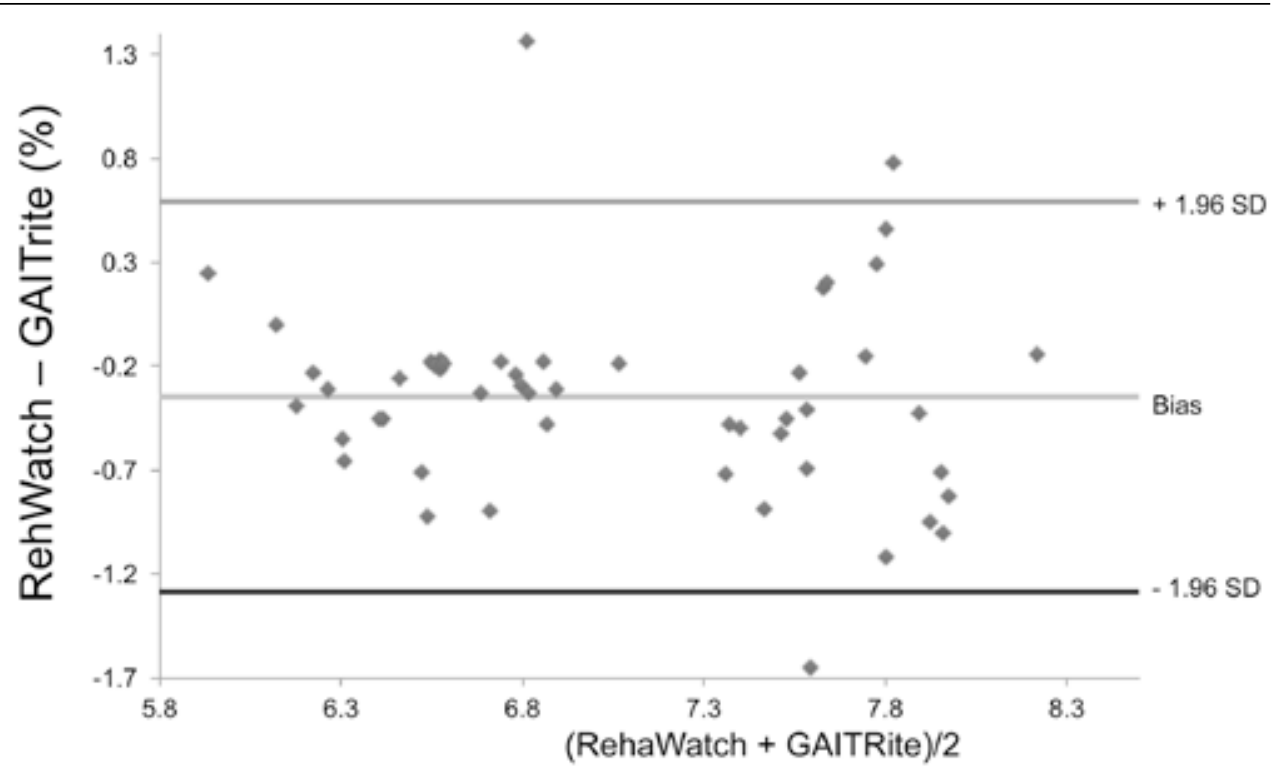

Fig. 3f: step length right leg [antilog] normal walking during dual-task 


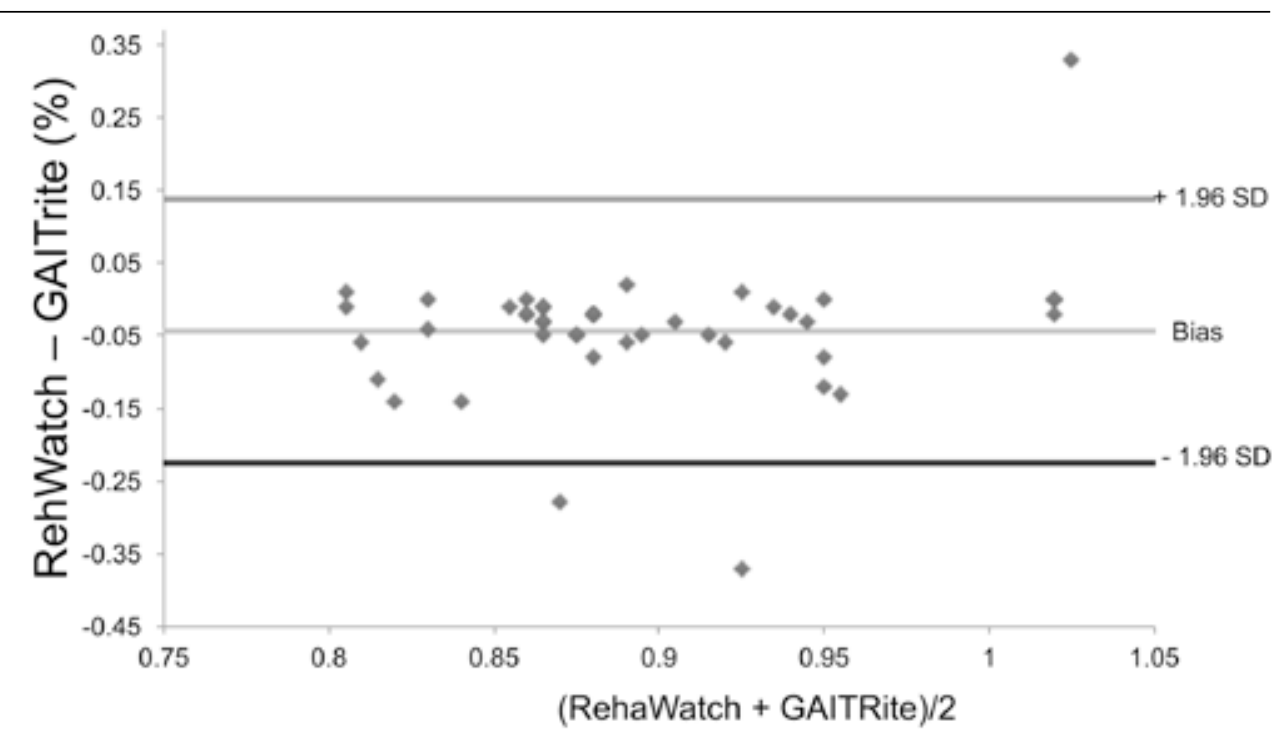

Fig. 4a: step duration time left leg [antilog] during fast walking during single-task

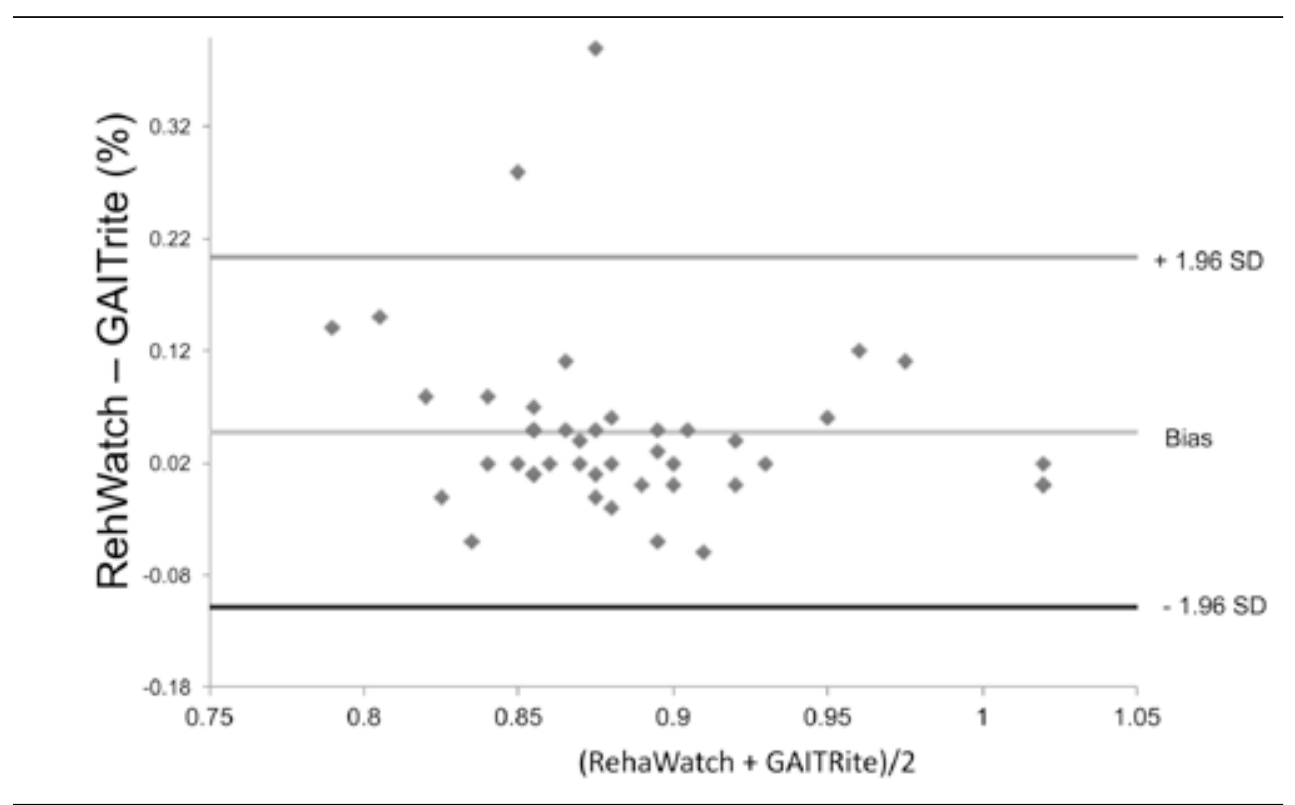

Fig. 4b: step duration time right leg [antilog] during fast walking during single-task 


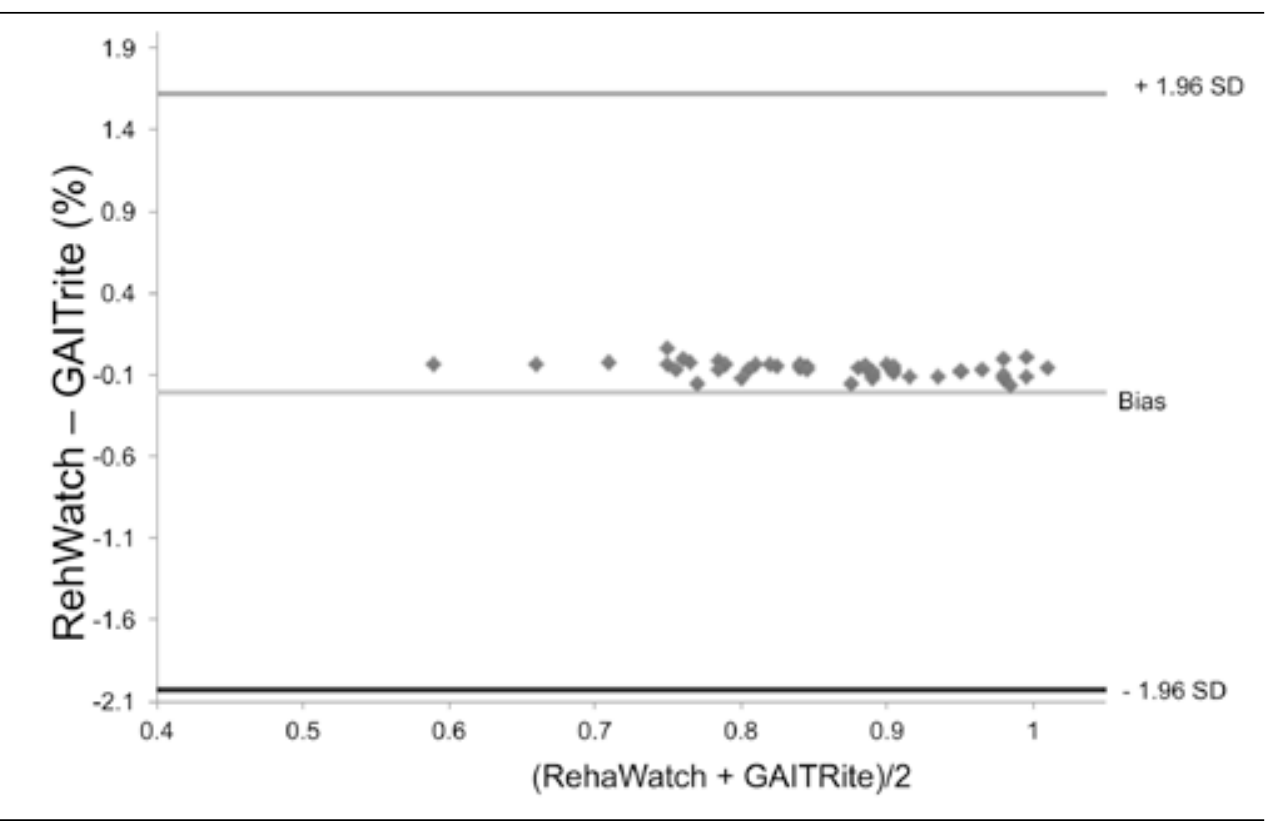

Fig. 4c: velocity [antilog] during fast walking during single-task

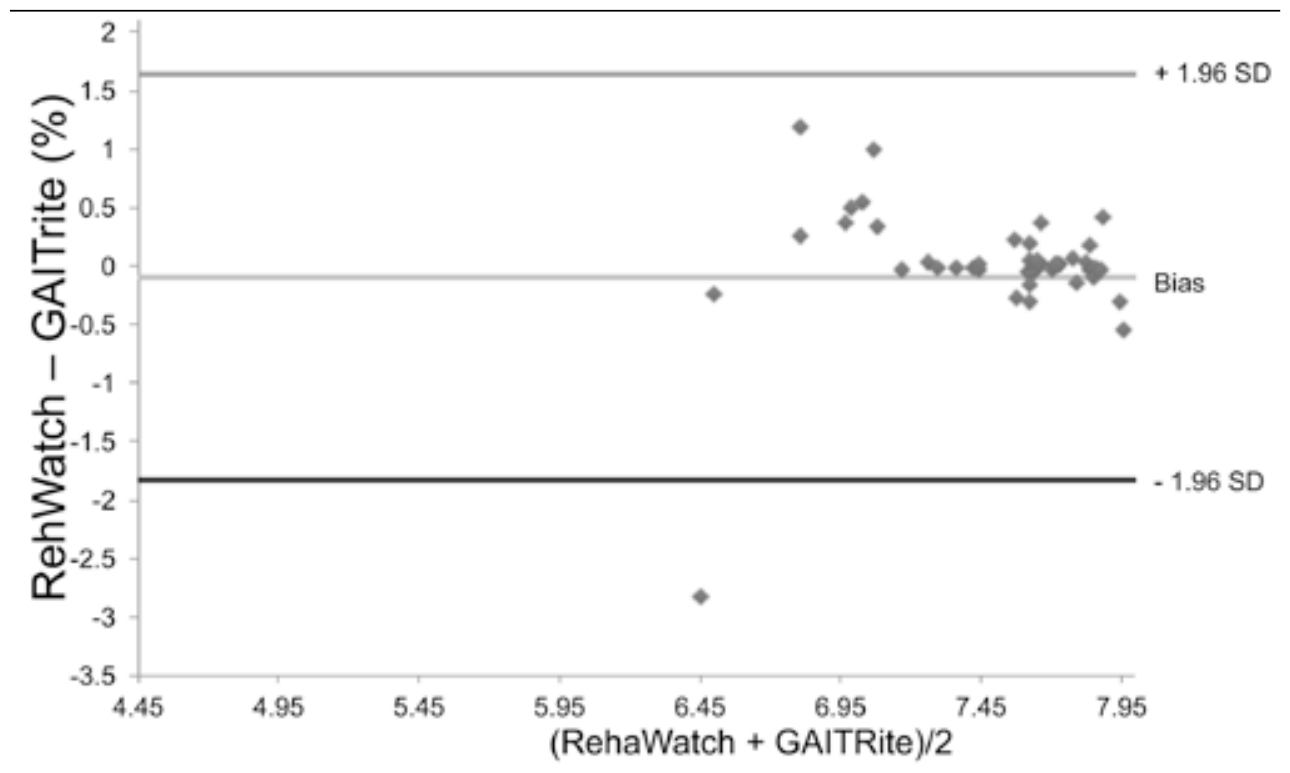

Fig. 4d: cadence [antilog] during fast walking during single-task 


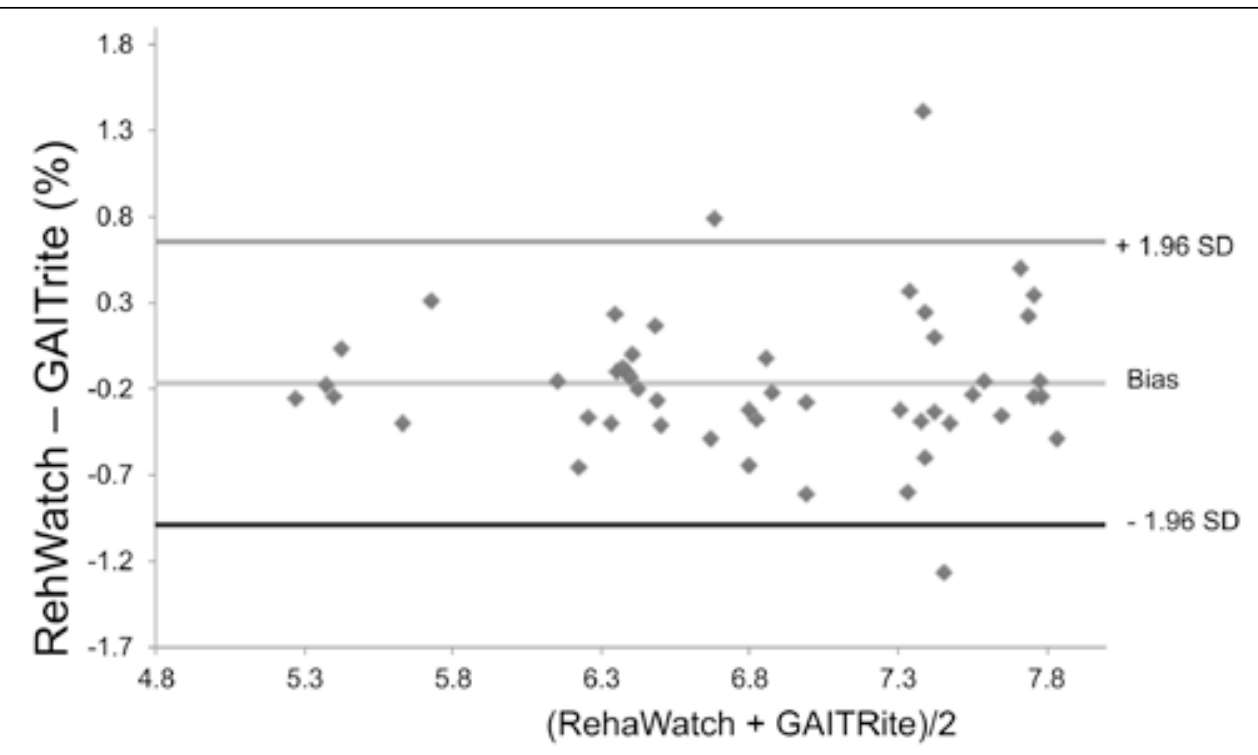

Fig. 4e: step length left leg [antilog] during fast walking during single-task

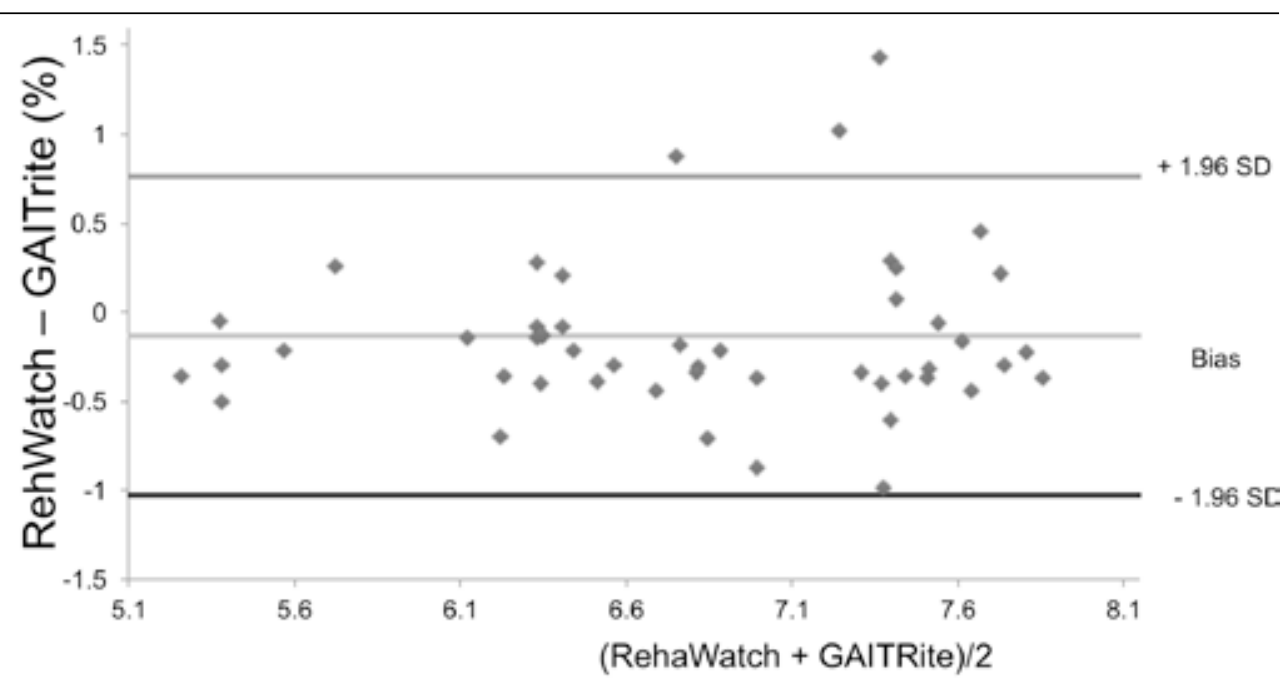

Fig. 4f: step length right leg [antilog] during fast walking during single-task 


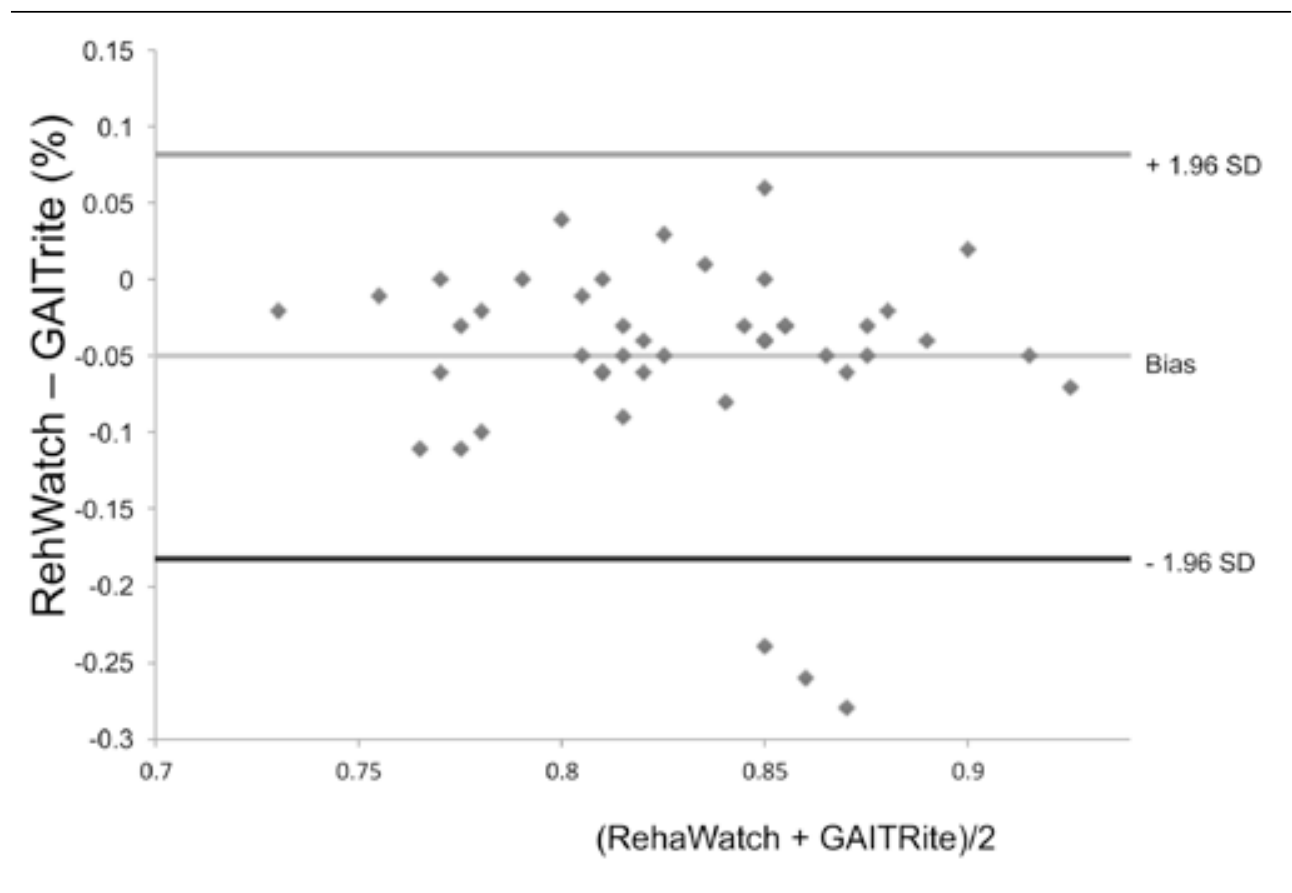

Fig. 5a: step duration time left leg [antilog] during fast walking during dual-task

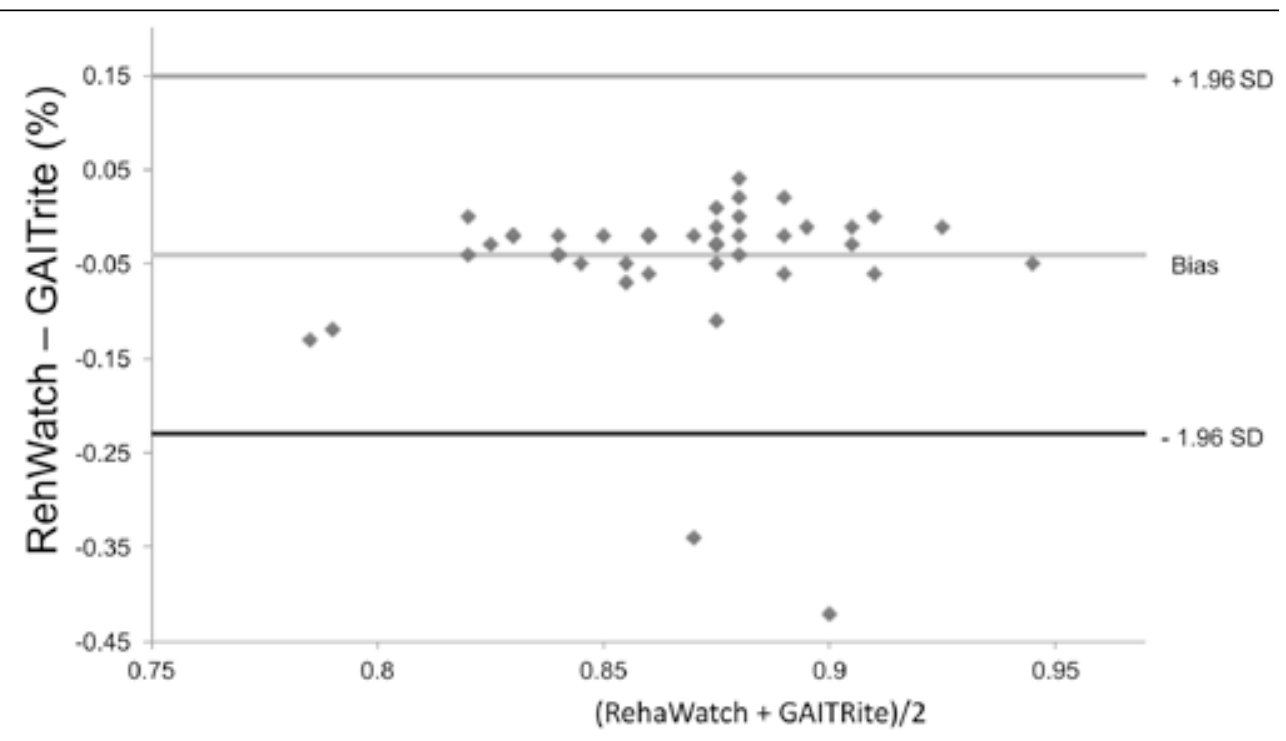

Fig. 5b: step duration time right leg [antilog] during fast walking during dual-task 


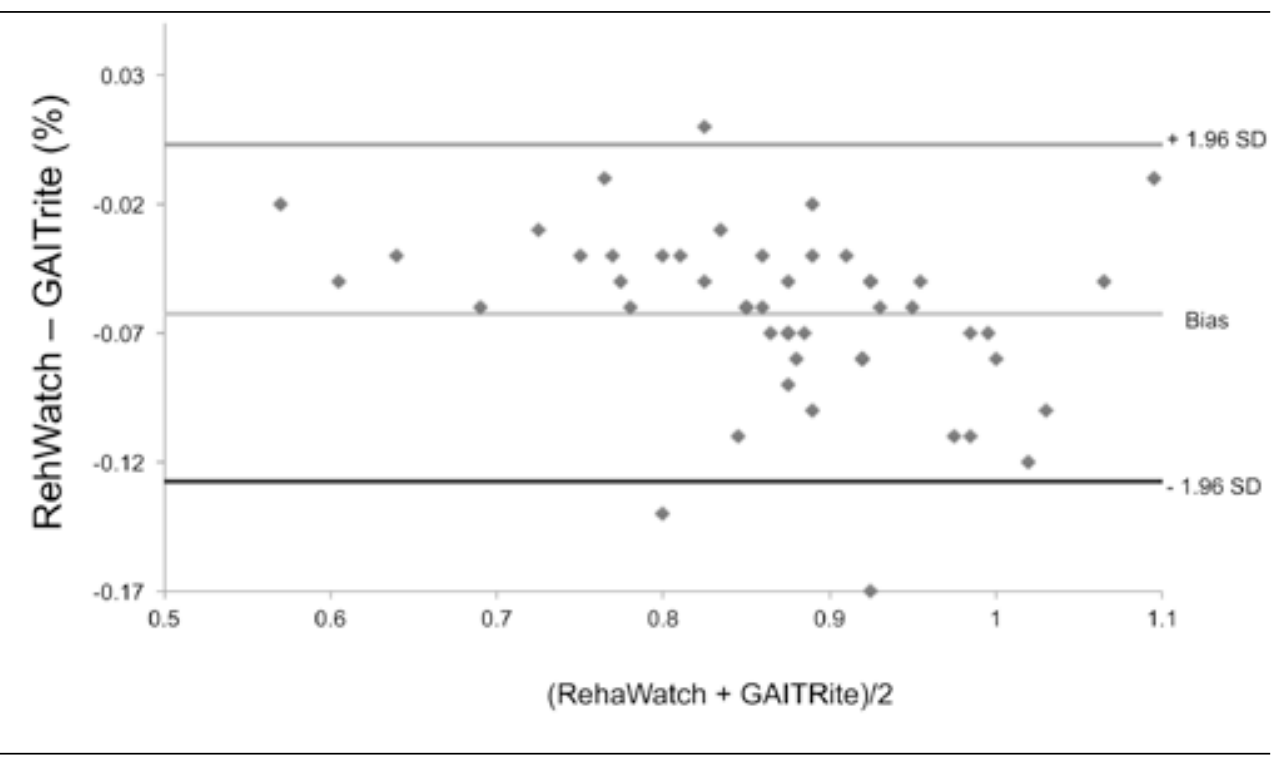

Fig. 5c: velocity [antilog] during fast walking during dual-task

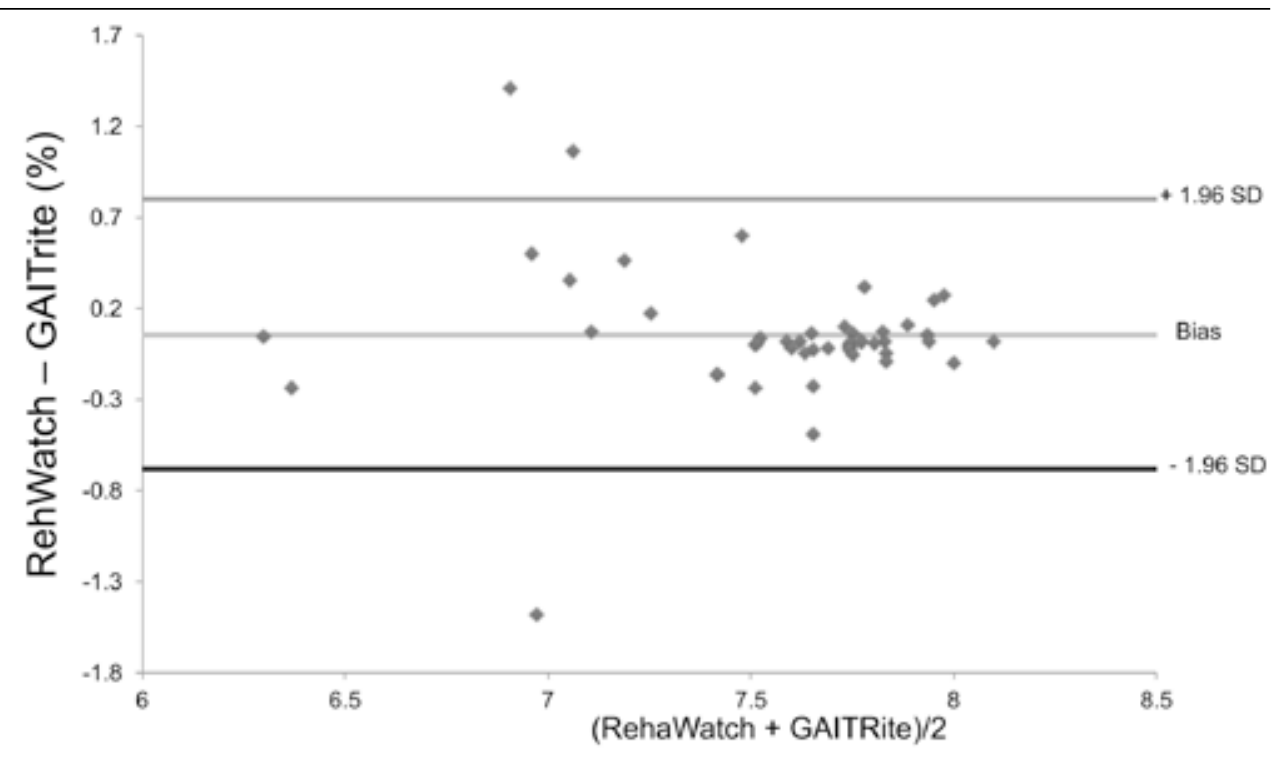

Fig. 5d: cadence [antilog] during fast walking during dual-task 


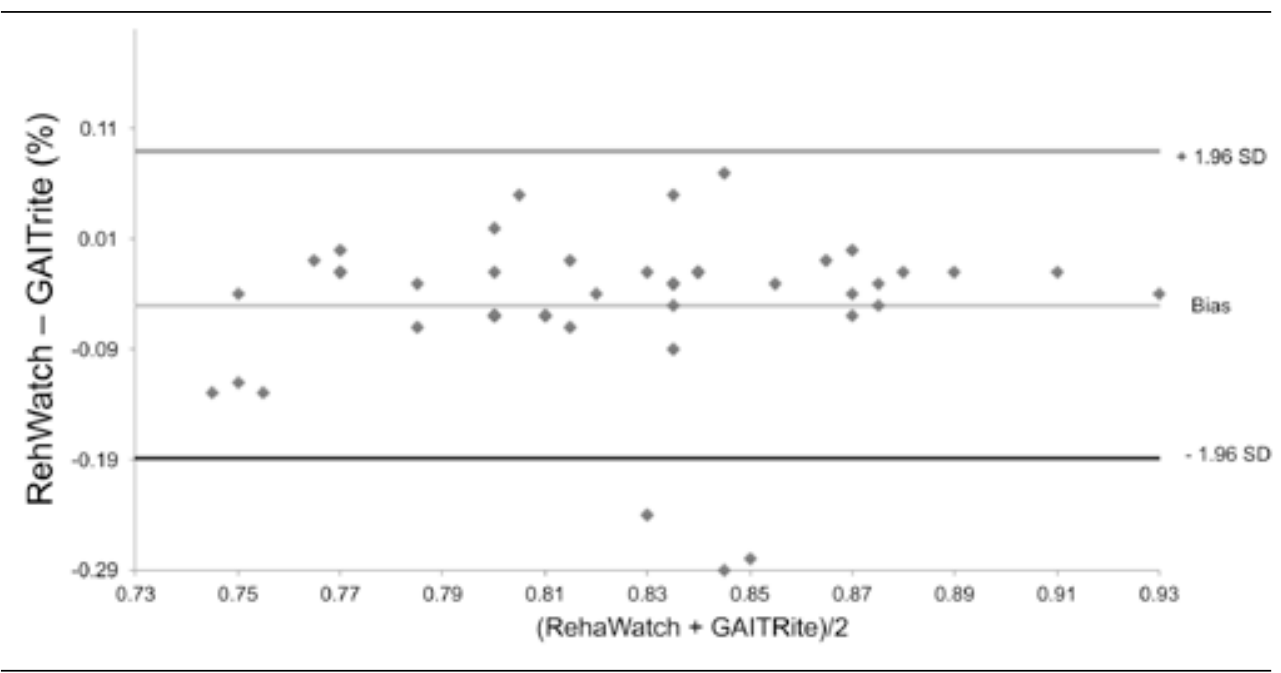

Fig. 5e: step length left leg [log] during fast walking during dual-task

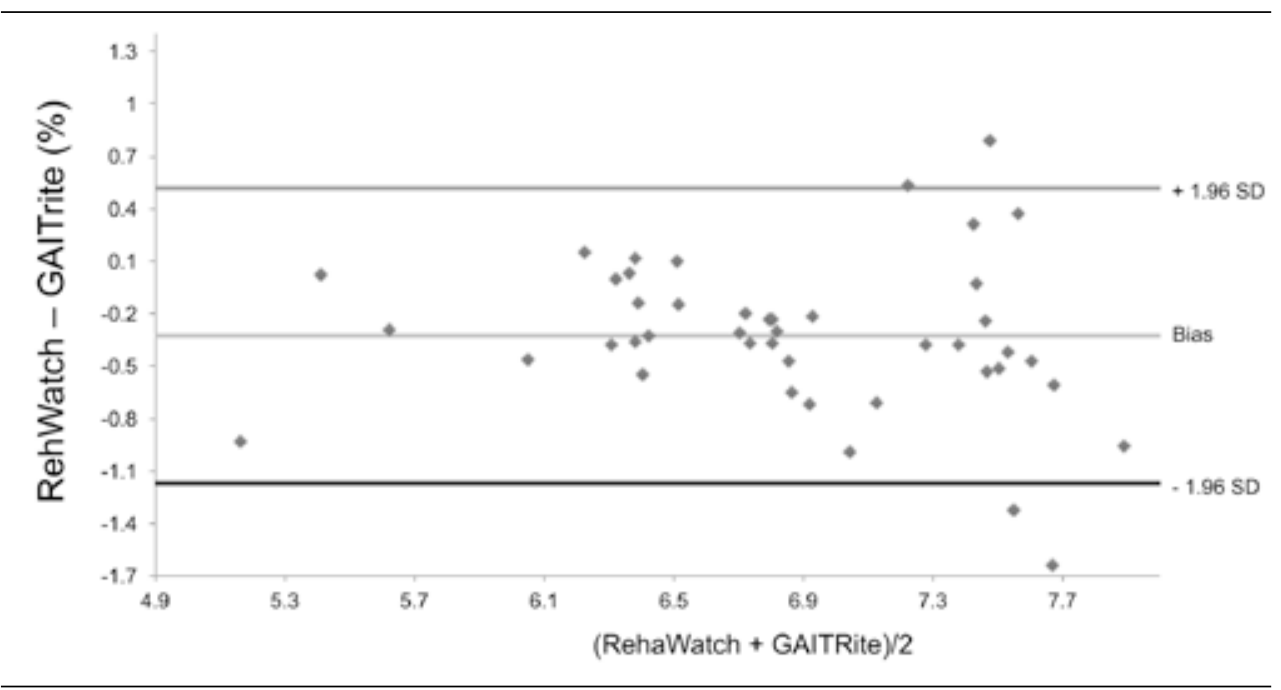

Fig. 5f: step length right leg [antilog] during fast walking during dual-task 


\section{Discussion}

The aim of this study was 1) to determine the concurrent validity of a shoe mounted wearable gait assessment system (RehaWatch ${ }^{\circledR}$ ) with the GAITRite ${ }^{\circledR}$ system for spatial-temporal gait parameters that were recorded for averaged step data in a frail older adult population living in long term care and 2) to compare the levels of agreement (LOA) for averaged step data from different walking conditions; e.g. single and dual task walking while walking at preferred and fast speed.

The concurrent validity for averaged step data was at most moderate for the cadence variable and differences exceeded the a-priori set $7 \%$ value. The systematic biases of the gait measures were significant $(p<0.05)$, with mostly weak ICC values, thus questioning the validity of the foot attached measurement approach in our sample. These biases seem to be related to the spatialtemporal variable estimations, which were different according each measurement system.

These results are at variance with a study comparing the RehaWatch ${ }^{\circledR}$ gait analysis system with treadmill walking at different speeds [38] in a sample of healthy participants with a mean age of 31 years. Although in this study the RehaWatch ${ }^{\circledR}$ system also overestimated most variables compared to the treadmill walking, it did so with high ICC values. Bland Altman Plots of the Cadence measure in this study [38] showed a systematic error since both repeatability coefficients were on the same side of zero [37] as opposed to our results.

It is known that different measurement methods and devices do not always exactly agree [39]. However, clinicians want to know whether new devices might be used interchangeably for the performance of gait analysis. Our study revealed that the RehaWatch ${ }^{\circledR}$ gait analysis system cannot be used interchangeably with the GAITRite ${ }^{\circledR}$ system. Gait analysis systems should provide valid information describing gait characteristics [40] in order for clinicians to be able and interpret results of an assessment.

Our results are partly in line with a study from Derlien et al. [41] who investigated the concurrent validity of the RehaWatch ${ }^{\circledR}$ compared to the medilogic ${ }^{\circledR}$ footswitch system. The gait parameters investigated were double stance length $(\mathrm{m})$, double stance time $(\mathrm{s})$ and velocity $(\mathrm{m} / \mathrm{s})$ during normal walking. The participants were healthy and between 20 and 30 years old. The results of our study agree with Derlien et al. [41] for the step length measure. However, the approach used by Derlien and co-workers focused on establishing measures of validity based on Pearson correlations which is not an appropriate methodological approach for a validity study [42].

Neegard et al. [43] performed two gait trials with stroke patients on a treadmill. They compared step length derived from the RehaWatch ${ }^{\circledR}$ with an ultrasonic measurement as a reference system. They showed that the step length was estimated quite accurately, lying between $3 \%$ and $5 \%$. This value is lower than the step length values (>10\%) of our present study. We assume that this is 
because the GAITRite ${ }^{\circledR}$ has pressure sensors and can, therefore, determine step length more accurately.

The cadence parameter showed concurrent validity for walking during single- and dual-tasking between RehaWatch ${ }^{\circledR}$ and GAITRite ${ }^{\circledR}$. Schwenk et al. [8] reported that temporal parameters of gait identified prefrail individuals and spatial parameters identified frail elderly individuals. Therefore, in clinical practice step length derived with the RehaWatch ${ }^{\circledR}$ system might possibly be used to discriminate between non-frail and pre-frail older adults. Further research is needed, however, to support or refute this assumption.

Poor concurrent validity is shown for the RehaWatch ${ }^{\circledR}$ for all outcome variables during singletask and dual-task fast walking. This is based on the large range of limits of agreement of all variables, increasing variability of the measurements during fast walking during single-task (step duration time left and right), heterogeneous distribution (step duration time left and right: dualtask condition and velocity during both conditions) and heteroscedasticity for step length during both conditions. The findings of the two systems are not interchangeable for fast walking during single-task and dual-task.

Factors that may have contributed to the observed differences may be due to the different quantities measured by the two gait analysis systems [38]. The RehaWatch ${ }^{\circledR}$ measures acceleration and angular velocity of the foot while the GAITRite ${ }^{\circledR}$ system is measuring pressure distribution under the feet. These different quantities each require that specific algorithms are employed to calculate gait events and that may be differently affected by factors such as foot placement [38]. Slight differences in these definitions may cause large unsystematic differences in gait characteristics between the two systems, especially in frail populations that are known to exhibit large variability in gait [44]. The double integration of the acceleration signal may affect the calculation of spatio-temporal gait characteristics and hence explain the resulting lack of validity in this population. Although potential drift is offset during zero acceleration phases of the sensor, because of slow shuffling walking patterns in many frail older adults there might still be a relative large effect, and even small step-to-step variability in foot placement would contribute to inaccuracies.

\section{Limitation}

A limitation of this concurrent validity study is the time schedule of the four walking conditions. The participants are LTC elderly and therefore not resilient. It would be better to distribute the measurements over two days. Another point is that elderly with mobility disability cannot perform fast walking. They seem unable to cope with the request to walk fast. A further limitation is the GAITRite ${ }^{\circledR}$ sensor length of $7.32 \mathrm{~m}$, which is relatively short. To obtain clear results, a longer 
distance should be used. Furthermore, the sample size is relatively small. Additional nursing homes should be included to obtain better results.

\section{Conclusion}

The accelerometric system was deemed not valid for the evaluation of spatial-temporal gait parameters step duration time, velocity, cadence and step duration in long term care dwelling older adults.

\section{Acknowledgement}

We would like to thank Ross Bennie for proofreading

\section{Conflict of interest}

None of the author has any financial or personal conflict of interest in relation to the submission article, or other any organizations or people 


\section{References}

1. Altman DG, Bland JM (1999) How to randomise. Bmj 319:703-704

2. Altman DG, Bland JM (1983) Measurement in medicine: the analysis of method comparison studies. The Statistician 32:307-317

3. Anstey KJ, Von Sanden C, Luszcz MA (2006) An 8-year prospective study of the relationship between cognitive performance and falling in very old adults. Journal of the American Geriatrics Society 54:1169-1176

4. Beauchet O, Herrmann FR, Grandjean R et al. (2008) Concurrent validity of $\operatorname{SMTEC}((\mathrm{R}))$ footswitches system for the measurement of temporal gait parameters. Gait Posture 27:156-159

5. Bilney B, Morris M, Webster K (2003) Concurrent related validity of the GAITRite walkway system for quantification of the spatial and temporal parameters of gait. Gait \& posture 17:68-74

6. Bland JM, Altman DG (1986) Statistical methods for assessing agreement between two methods of clinical measurement. Lancet 1:307-310

7. Bossuyt PM, Reitsma JB, Bruns DE et al. (2003) Towards complete and accurate reporting of studies of diagnostic accuracy: the STARD initiative. Bmj 326:41-44

8. Bossuyt PM, Reitsma JB, Bruns DE et al. (2003) The STARD statement for reporting studies of diagnostic accuracy: explanation and elaboration. Annals of internal medicine 138:W1-12

9. Bossuyt PM, Reitsma JB, Standards for Reporting of Diagnostic A (2003) The STARD initiative. Lancet 361:71

10. Brandes M, Zijlstra W, Heikens S et al.
(2006) Accelerometry based assessment of gait parameters in children. Gait Posture 24:482-486

11. Brauer SG, Woollacott M, Shumway-Cook A (2001) The interacting effects of cognitive demand and recovery of postural stability in balance-impaired elderly persons. The journals of gerontology. Series A, Biological sciences and medical sciences 56:M489496

12. Clegg A, Young J, lliffe S et al. (2013) Frailty in elderly people. Lancet 381:752-762

13. Davis lii RB, Õunpuu S, Tyburski D et al. (1991) A gait analysis data collection and reduction technique. Human Movement Science 10:575-587

14. De Bruin ED, Hartmann A, Uebelhart D et al. (2008) Wearable systems for monitoring mobility related activities in older people; a systematic review. Clinical Rehabilitation in press

15. Derlien $S$, Böhme $B$, Leistritz $L$ et al. (2010) Validitätsuntersuchung zum neuen, innovativen Ganganalysesystem RehaWatch von Hasomed. Manuelle Medizin 48:254-259

16. Donath L, Faude O, Lichtenstein E et al. (2016) Validity and reliability of a portable gait analysis system for measuring spatiotemporal gait characteristics: comparison to an instrumented treadmill. Journal of neuroengineering and rehabilitation 13:6

17. Fortune E, Lugade V, Morrow M et al. (2014) Validity of using tri-axial accelerometers to measure human movement - Part II: Step counts at a wide range of gait velocities. Medical engineering \& physics 36:659-669 
18. Grouven U, Bender R, Ziegler A et al. (2007) [Comparing methods of measurement] Dtsch Med Wochenschr 132 Suppl 1:e69-73

19. Hamacher D, Singh NB, Van Dieen JH et al. (2011) Kinematic measures for assessing gait stability in elderly individuals: a systematic review. Journal of the Royal Society, Interface / the Royal Society 8:1682-1698

20. Hartmann A, Luzi S, Murer K et al. (2009) Concurrent validity of a trunk tri-axial accelerometer system for gait analysis in older adults. Gait \& posture 29:444-448

21. Hartmann A, Murer K, De Bie RA et al. (2009) The effect of a foot gymnastic exercise programme on gait performance in older adults: a randomised controlled trial. Disability and rehabilitation 31:21012110

22. Hartmann A, Murer $\mathrm{K}$, De Bie RA et al. (2010) The effect of a training program combined with augmented afferent feedback from the feet using shoe insoles on gait performance and muscle power in older adults: a randomised controlled trial. Disability and rehabilitation 32:755-764

23. Hartmann A, Murer K, De Bie RA et al. (2009) Reproducibility of spatio-temporal gait parameters under different conditions in older adults using a trunk tri-axial accelerometer system. Gait \& posture 30:351-355

24. Henriksen $\mathrm{M}$, Lund $\mathrm{H}$, Moe-Nilssen $\mathrm{R}$ et al. (2004) Test-retest reliability of trunk accelerometric gait analysis. Gait Posture 19:288-297

25. Kavanagh JJ, Menz HB (2008) Accelerometry: A technique for quantifying movement patterns during walking. Gait Posture 28:1-15
26. Lord S, Rochester L, Baker K et al. (2008) Concurrent validity of accelerometry to measure gait in Parkinsons Disease. Gait Posture 27:357-359

27. Maffiuletti NA, Gorelick M, Kramers-De Quervain I et al. (2008) Concurrent validity and intrasession reliability of the IDEEA accelerometry system for the quantification of spatiotemporal gait parameters. Gait Posture 27:160-163

28. Mansfield A, Lyons GM (2003) The use of accelerometry to detect heel contact events for use as a sensor in FES assisted walking. Med Eng Phys 25:879-885

29. Martinez-Ramirez A, Martinikorenal, Gomez $M$ et al. (2015) Frailty assessment based on trunk kinematic parameters during walking. Journal of neuroengineering and rehabilitation 12:48

30. Menz HB, Latt MD, Tiedemann A et al. (2004) Reliability of the GAITRite walkway system for the quantification of temporospatial parameters of gait in young and older people. Gait \& posture 20:20-25

31. Moe-Nilssen R (1998) Test-retest reliability of trunk accelerometry during standing and walking. Arch Phys Med Rehabil 79:13771385

32. Montero-Odasso M, Muir SW, Hall M et al. (2011) Gait variability is associated with frailty in community-dwelling older adults. The journals of gerontology. Series A, Biological sciences and medical sciences 66:568-576

33. Negaard N-O-, Kauert RA, S., Schauer T et al. (2005) Gait phases detection and step length estimation of gait by means of inertial sensors. In: 3rd European Medical \& Biological Engineering Conference (EMBEC 2005). Prague, Czech Republic 
34. Ritt M, Schülein S, Lubrich $\mathrm{H}$ et al. (2016) High-technology based gait assessment in frail people: Associations between spatio-temporal and three-dimensional gait characteristics with frailty status across four different frailty measures. The journal of nutrition, health \& aging:8

35. Rueterbories J, Spaich EG, Larsen B et al. (2010) Methods for gait event detection and analysis in ambulatory systems. Medical engineering \& physics 32:545-552

36. Schwenk M, Howe C, Saleh A et al. (2014) Frailty and technology: a systematic review of gait analysis in those with frailty. Gerontology 60:79-89

37. Schwesig R, Kauert R, Wust S et al. (2010) [Reliability of the novel gait analysis system RehaWatch]. Biomedizinische Technik. Biomedical engineering 55:109-115

38. Schwesig R, Leuchte $\mathrm{S}$, Fischer $\mathrm{D}$ et al. (2011) Inertial sensor based reference gait data for healthy subjects. Gait \& posture 33:673-678

39. Verghese $J$, Buschke $H$, Viola $L$ et al (2002) Validity of divided attention tasks in predicting falls in older individuals: a preliminary study. Journal of the American Geriatrics Society 50:1572-1576

40. Webster KE, Wittwer JE, Feller JA (2005) Validity of the GAITRite walkway system for the measurement of averaged and individual step parameters of gait. Gait \& posture 22:317-321

41. Woollacott M, Shumway-Cook A (2002) Attention and the control of posture and gait: a review of an emerging area of research. Gait \& posture 16:1-14

42. Woollacott MH, Tang PF (1997) Balance control during walking in the older adult: research and its implications. Phys Ther

\section{7:646-660}

43. Zijlstra W (2004) Assessment of spatiotemporal parameters during unconstrained walking. Eur J Appl Physiol 92:39-44

44. Zijlstra W, Hof AL (2003) Assessment of spatio-temporal gait parameters from trunk accelerations during human walking. Gait Posture 18:1-10 



\section{Chapter 9}

General Discussion 


\section{Introduction}

Reaching old age in good health is an important individual and social goal because it potentially allows the elderly to prolong their independent lifestyle. Epidemiological research confirmed that a well-established support of physical activity for the elderly can prevent precocious decline of body structures and body function, resulting in more independency and preservation of health [1]. This could also reduce the pressure on healthcare services and budgets [2].

Zeyfang und Braun [3] proposed how physical functions in the elderly population should be classified into three categories: 1. "Go-Go" = independent elderly individuals, 2. "Slow-Go" = needy elderly individuals with a slight handicap, 3. "No-Go" = elderly individuals in need of care with severe functional limitations, such as frail elderly and elderly with mobility disabilities. Due to a varying initial level of physical performance between Go-Go, Slow-Go and No-Go elderly individuals, training programs should be designed differently and adapted to individual needs.

However, evidence of training programs for No-Go elderly in need-of-care is scarce. One reason is that elderly in need-of-care have often been excluded from research studies and therefore few empirical evidence exists about the effects of exercise training in these individuals [4].

The main findings of this Doctoral Thesis were that 1) whole-body vibration is a safe training method, and 2) the combination of whole-body vibration and exergame could use as skilling-up training regimes in No-Go elderly.

\section{Findings}

\subsection{Effects of whole-body vibration (WBV)}

Three types of WBV are used in clinical settings and research: vertical sinusoidal whole-body vibration (VS-WBV), side-alternating sinusoidal whole-body vibration (SS-WBV) and stochastic resonance whole-body vibration (SR-WBV) [5-7]. Both systematic reviews (Chapters 2 and 3) analyzed the effects of VS-WBV and SS-WBV on muscle strength and postural control in elderly individuals. Chapter 3 included SR-WBV into the analysis.

These two studies showed evidence that elderly (mainly Go-Go and Slow-Go) individuals may benefit more from SS-WBV to improve balance as compared to VS-WBV. But a high risk of bias and heterogeneity of the included studies hampered a clear-cut conclusion in terms of effects on static, dynamic and functional balance. Further studies reported that a sensimotor WBV training may be effective in enhancing basic balance ability and mobility among the elderly, particularly in frail persons [8]. It may improve muscle strength and balance compared to a control group but not to conventional exercise [9]. None of the studies separately assessed the effects of SS-WBV 
and VS-WBV on static, dynamic and functional balance.

The second review presented in Chapter 3 of this thesis covered the vibration types VS-WBV, SS-WBV and SR-WBV, as well as the functional classification Go-Go, Slow-Go and No-Go. Moderate evidence for isometric maximum voluntary contraction (IMVC) as well as for dynamic strength after VS-WBV in Go-Go were determined. SS-WBV presented moderate evidence for improvement of IMVC, for power, for rate of force development, and for functional strength. The analysis for SR-WBV for Slow-Go showed high evidence for increased functional strength while in No-Go persons only a moderate evidence for improvement was found.

These results show beneficial effects after WBV intervention, mainly in the No-Go group. These findings illustrate that WBV can be used as skilling-up exercise in those participants which are not able to perform traditional exercises. Previously published reviews from Lau et al. [10] and Osawa et al. [11] described beneficial effects on muscle strength after WBV in an elderly population. But they did not classify elderly into Go-Go, Slow-Go and No-Go, nor discriminate between the WBV types VS-WBV, SS-WBV and SR-WBV. Furthermore, they did not pool different strength outcome variables in the same meta-analysis such as isometric muscle strength, isokinetic muscle strength, leg extension or rate of force development. Our systematic review was able to present more accurate results regarding strength effects after WBV intervention in elderly individuals compared to the review and meta-analysis of Lau et al. [10] and Osawa et al. [11].

We are well aware that the meaningfulness of our systematic reviews and meta-analysis regarding external validity could be limited by aspects such as missing intervention protocols, missing reporting standards and different treatment frequencies or different follow-up periods. It is advisable to produce more high quality studies with follow-up periods. The aim should be registered and reporting studies which preferably adhere to guidelines; e.g. the CONSORT guideline [12], World Health Organization clinical trial register and using standardized recommendation to the reporting of WBV training regimes [13].

The systematic reviews and the meta-analysis provided us with an overview of the current evidence on effects after WBV intervention on postural control and strength when planning the feasibility studies (Chapters 4 to 6) and main study (Chapter 7 ) of this thesis.

\subsection{Feasibility of whole-body vibration}

This study revealed that the study protocol described in Chapters 4 and 5 was feasible and safe for Slow-Go elderly. In Chapter 4, an RCT design was chosen, in which one group received vibration and the other received rest. All Slow-Go elderly $(n=10)$ in the SR-WBV group tolerated a single bout SR-WBV over five sets of one-minute vibration at $5 \mathrm{~Hz}$, Noise 4 with one-minute rest in between.

The next step was to extend the training program. For this purpose, a randomized crossover design was selected (Chapter 5). Twenty Slow-Go elderly were included and assigned to group 
A or group B. Group A received an SR-WBV intervention with a frequency at $6 \mathrm{~Hz}$, Noise level 4 in period one (over four weeks, three times a week ( 5 sets, 60 seconds vibration, 60 seconds rest)) and then a sham intervention with a frequency at $1 \mathrm{~Hz}$, Noise level 1 (SR-WBV / Sham) in period 2 after a wash-out phase (16 days) or vice versa (Sham / SR-WBV). Seventeen participants completed the ten-week SR-WBV study. This long-term study seemed acceptable and safe for Slow-Go elderly and this study protocol was used for the next feasibility study (Chapter 6).

Chapter 6 showed that SR-WBV training in No-Go elderly is feasible and safe, but that the study methodology requires some major modifications. The findings in Chapter 6 were not in line with Chapters 4 and 5, probably because of the high attrition rate of $44.4 \%$. Sievänen et al. [14] reported an attrition rate of $13 \%$ while Zhang et al. [15] had $17 \%$ attrition in No-Go elderly. These attrition rates are in line with a reported drop-out rate of $6 \%$ to $34 \%$ that occur in the first three months of a study [16-18]. The reason for drop-out in our study (Chapter 6) was loss of interest because the participants aren't interesting enough for SR-WBV.

Fairhall et al. [19] postulated that frail elderly individuals should be encouraged and supported if they are to adhere to an intervention plan. Sievänen et al. [14] individually escorted the participants to training sessions. These elements need further attention. We address this topic in Chapter 7. This study was based on the motivation-volition process model whereas health professionals coached the recruitment process and the training process in order to prevent attrition.

\subsection{Innovation training program}

The study protocol of the main study (Chapter 7) was based on the findings of Chapter 6. The intervention was planned as an adaptation of the Falls Management Exercise program (FaME) [20]. An eight-week skilling-up period at the beginning of the intervention was used instead of the 12-week period proposed previously by Skelton and Dinan [20]. Kawanabe et al. [21] specified that effects of WBV training in the elderly may be expected after an intervention period of two months. Shorter duration of the intervention is pragmatic (less resources needed) and may increase adherence. Furthermore, to maintain participants' compliance in the study, a motivation-volition process model was applied. This model assists persons to set up and maintain or improve their physical activity due to strategies and self-control for the realization of their intentions regarding function and independence. In addition, the participants were transferred from their rooms and back again.

The training regime followed in this intervention study consisted of SR-WBV and VG. In addition to sensimotor training, cognitive elements should be a part of training regimes for elderly individuals since falls often occur during attention-demanding circumstances [22]. Some studies showed evidence that VG is beneficial for functional capacities such as gait speed and risk of falls in elderly individuals [23-27]. 
The application of the modified FaME for the main study (Chapter 7) yielded a good adherence rate $(100 \%)$ and attrition rate $(0 \%)$ and significant differences on the Short Physical Performance Battery test $(p=0.001)$ and on strength values $(p<0.05)$ between IG and SG in LTC elderly. Strength variables were: isometric maximum voluntary contraction (IMVC) of right and left knee extensors and knee flexors, isometric rate of force development (IRFD) of the knee extensors and hamstrings. In addition, sub-values (Fsub) of isometric maximum voluntary contraction at $30,50,100$ and $200 \mathrm{~ms}$ and sub-values of isometric rate of force development (IRFDsub) in the range between 0-30, 0-50, 0-100 and 100-200 ms were recorded. This study showed that SRWBV and VG may be used as a skilling-up exercise for LTC elderly in need-of care.

Manini and Clark [28, 29] described how aging is associated with loss of muscle strength (dynapenia) and motor function [30]. Increase and decrease in strength are caused by neurological and skeletal muscle factors [28]. Traditional strength exercise improves muscle strength as a result of an improved neural drive and muscle hypertrophy [31-33]. In addition, sensimotor training has a large influence on the neuromuscular system at the initiation of production of rate of force development and neuromuscular activation at the onset of voluntary actions [34-37]. The innovative training program developed in the present study shows all features of a sensimotor training program for skilling-up LTC elderly in need-of care. For activities in daily life, rate of force development and explosive strength are of uppermost importance [38]. These strength components are improved by SR-WBV [39]. Kessler et al. [39] found an improvement with high effect sizes $(p=0.064 ; r=0.51)$ after four week SR-WBV on rate of force development of the $\mathrm{m}$. quadriceps in LTC elderly $(n=11)$.

Until now, there have been few studies that combined VG and treatment/exercises as a training regime in an elderly population. Kramer et al. [40] investigated the effect of 61 patients (mean age: $47 \pm 9$ years) with multiple sclerosis. They divided the participants into conventional balance training group, single-task exercise on a unstable platform group and a combination of exergaming (Wii Sports/Sports Resort/Fit games) with balance exercise group. They observed a significantly higher improvement in Dual Task condition during gait test to conventional balance training group and single-task exercise on a unstable platform group. De Bruin et al. [41] postulated in their theoretical essay that VG could enhance motivation, thereby promoting adherence and reducing attrition.

Our findings demonstrated that SR-WBV and VG were feasible and effective training methods for LTC elderly in need of care, leading to significant improvements in performance and strength. This study opens new perspectives for clinicians and researchers in the field of LTC elderly in need of care (No-Go).

\subsection{Validation study}

Chapter 8 describes a validation study of a new mobile gait analysis system (RehaWatch ${ }^{\circledR}$ 
system, Hasomed Magdeburg, Germany) used in clinical daily routine. Its validity and reliability was shown in young persons ( $n=51$, mean age: 24 years) [42], but this information is lacking in the elderly. Schwenk et al. [43] concluded that changes in gait parameters during walking in prefrail and frail elderly individuals is sufficiently clarified. Therefore, we evaluated the validity of the RehaWatch ${ }^{\circledR}$ system in 23 elderly persons with a mobility disability (mean age: 90.90 \pm 8.4 years). The GAITRite ${ }^{\circledR}$ system (CIR Systems Inc., Havertown, USA) was chosen as a reference standard. The study results showed that the RehaWatch ${ }^{\circledR}$ system has a very low validity. Only cadence (expressed in number of steps / walking time) is interchangeable with other devices that assess gait parameters. Other variables, such as stance duration left leg and right leg (expressed in s), walking velocity and cadence (number of steps divided by walking time) for temporal parameters and for spatial parameters, step length left and right $(\mathrm{cm})$ are not interchangeable (not valid). This validation study differs from the one published by Schwesi et al. [44]. They evaluated the validity of the RehaWatch ${ }^{\circledR}$ system in healthy people during one walking trial (normal walking). In contrast, our validation study included four different walking trials: normal walking with and without counting and fast walking with and without counting in LTC elderly. Additionally, in Chapter 8 it was determined that LTC elderly could not walk faster as their normal walking speed.

\section{Limitations and methodological considerations}

Limitations of the different studies included in this Doctoral Thesis were discussed in each of the Chapters 2 through 8 separately. However, further reflection on aspects of validity, methodological and theoretical issues of this thesis is needed to specify whether the impact of SR-WBV alone and as a combined training program (SR-WBV plus VG) and the validity of the RehaWatch ${ }^{\circledR}$ system are accurately characterized and whether results can be generalized.

\subsection{Validity}

Systematic reviews generally depend on the methodological quality of the included studies. In the systematic review and the meta-analysis (Chapters 2 and 3) in this Thesis, only randomized controlled trials (RCT) have been included. Estimating quality and sensitivity to bias is essential when interpreting studies and conducting systematic reviews and meta-analyses. Various appraisal tools exist that include checklists and scales as well as component systems. But they are seldomly evaluated [45]. To assess the internal validity of the included studies we used the risk-of-bias-tool (RoB) of the Cochrane Collaboration. The Cochrane Collaboration RoB is recommended when evaluating quality appraisal of RCT [46]. Hartling et al. [47] showed in their research review that the reliability between two reviewers was fair for most items ( $\mathrm{K}=$ $0.24-0.37$ ). They assessed the risk of bias for 154 RCTs. They found that differences in the evaluation of RoB were caused by differences in the interpretation of the RoB instrument. We used the RoB instrument of the Cochrane Collaboration because of its transparency, recognition 
of the principal types of bias, good item handling and the globally understandable graphical presentation of results. In order to achieve a good agreement, a standardized approach was conducted, with clear and detailed operationalization of the various quality parameters.

At the moment that the first two feasibility intervention studies of this Doctoral Thesis were launched (Chapters 4 and 5), no studies evaluating the effect of SR-WBV on strength, balance or physical performance in an elderly population were available. For this reason, the intervention was determined from training programs used in other fields. The feasibility studies presented in Chapters 4 and 5 used the newly postulated concept. Schmidtbleicher et al. [48-55] focused predominantly on Parkinson's disease patients.

Chapter 7 reported on a single blinded randomized controlled trial. In our study the participants were blinded to the intervention method while the examiner was not. If the outcome assessor is not blinded, there is a higher risk of assessment bias [56]. In most physiotherapy studies blinding the examiner and trial staff is problematic. In the feasibility studies included in this Doctoral Thesis, the aim was to blind participants, outcome assessor and trial personnel. While the blinding of the participants was maintained throughout the intervention period of six months, the blinding of the outcome assessor and trial personnel was not. The reason was that only one trial person was responsible for carrying out the intervention. At the end of the study period, sometimes the outcome assessor had to carry out the intervention. In our case, two trial persons would have been optimal. These two persons may have alternated or helped out in the event of illness of the other trial person. The feasibility study in Chapter 6 recruited two Bachelor students as trial personnel. In contrast, the main study presented in Chapter 7 only recruited one Master student as trial person.

Validity of the assessment instrument asks if the instrument really measures what it is supposed to measure [57]. To assess the validity of an instrument, the instrument should be compared to a reference (gold) standard instrument. In context of the study presented in Chapter 8 it was intended to assess the validity of spatio-temporal gait parameters of the RehaWatch ${ }^{\circledR}$ system in LTC elderly in need of care during normal and fast walking while single and dual tasking. The RehaWatch ${ }^{\circledR}$ system (Index System) was compared with the GAITRite ${ }^{\circledR}$ system (Reference System). Three-dimensional kinematic motion analysis systems are the reference (gold) standard for investigating spatio-temporal gait parameters. For pragmatic reasons of manageability and time resources (no fixing body marker and fixing camera) the GAITRite system was used as the reference standard instrument in this study. We realized that it would be additionally meaningful, in order to receive optimal data and to add further knowledge, to use three-dimensional kinematic motion analysis systems in future studies.

\subsection{Heterogeneity}

One of the primary goals of the medical services is to maintain independency in daily living in 
elderly individuals. Difficulties to reach this goal arise from the fact that each ageing process is individually different, and the elderly therefore develop a striking heterogeneity in terms of loss of independence, decreased life satisfaction and social isolation. Different needs and care requirements arise from certain life situations across elderly people. As a result of this striking heterogeneity, there is an increasing demand for and duration of health care services. This Thesis used the simple classification Go-Go, Slow-Go and No-Go [3] to evaluate function in elderly individuals.

Chapters 4 and 5 investigated effects after SR-WBV on Slow-Go elderly, while chapter 6 reported effects after SR-WBV on No-Goes. The inclusion criteria in Chapter 4 and 5 have been designed to cover a broad area. LTC begins around 75 to 85 years of age [58]. We chose a particularly low starting age. The age of the study participants included in Chapters 6 and 7 ranged from 70 to 100 years. Elderly individuals under age 70 years are able to process training stimuli better than those above 70 years of age. This is because people under 70 years of age usually have a better intact sensimotor system compared to person over 70 years of age. This should be taken into account for future studies and the inclusion age raised to above 75 years old.

We used the Physical Performance Battery Test [59] to assess the current function of the participants. This assessment seems to be suitable for this group of participants. However, it should be noted that LCT elderly are very heterogeneous. A further sub-classification of the NoGo group should be applied: LTC elderly in need of care should be sub-divided into: a) mobile elderly that are moving with aids, b) elderly that cannot move themselves and are dependent on a wheelchair, or c) elderly who are bedridden. This thesis includes item a) mobile elderly and item b) wheelchair elderly individuals. Wheelchair elderly are able to stand with aids, but cannot walk. We include two elderly individuals that were unable to walk and were dependent on a wheelchair. Future studies should focus on this sub-classification.

However, we have carried out for three feasibility studies presented in Chapters 4 to 6 to ensure an acceptable design studies and reduces limitation for the main study.

\subsection{Motivation aspect}

Physical activity guidelines for elderly individuals aged 65 or older recommend at least 150 minutes of moderate-intensity aerobic activity per week and muscle strengthening exercises on at least two days per week [60]. But it is known that only $58 \%$ of men and $52 \%$ of women aged $65-74$ years and $44 \%$ of men and $20 \%$ women aged $75-84$ years were achieving the recommended amounts of physical activity [61]. A European survey confirmed that among the elderly $40 \%$ show no interest in physical activity. They prefer to do other things [62]. This knowledge about adherence is in accordance with the compliance rate in our study that was described in Chapter 6. The adherence rate we had was $81.6 \%$ (98 of 120 training sessions) and the attrition rate was $58 \%$ ( $n=7$ drop-outs) from a total of 12 participants. 
Reasons for a lack of motivation are that people are not sufficiently interested in exercise, or the value of the outcomes is not considered enough to make it a priority in their lives [63]. It seems that the elderly included in the studies of Chapters 4 and 5 were motivated enough to participate and to adhere to the study program compared to the LTC elderly in need of care in the study presented in Chapter 6.

Changes in health behavior are not always easily accomplished or sustained [64]. The challenge is how best to support No-Go elderly in adopting training behaviors. Researchers who study behavior change agree that change is most likely when it is self-motivated and rooted in positive thinking [65]. The literature described two different lines of research, the social cognition models (Social Cognitive Theory [66], Theory of Planned Behavior [67]) and the action control theories health (Implementation Intentions Model [68]). The social cognition model emphasises more the motivational aspects that lead to the formation of behavioral intentions. The action control is more focused on volitional competencies that transform intentions into concrete actions.

Göhner and Fuchs [69] postulated that most physical activity intervention programs still do not explicitly address volitional planning. Therefore, we focused on the motivation-volition (MoVo) process model (Chapter 7) according to Fuchs [70]. The MoVo process model is an attempt to integrate most content of the social cognition-model and action control theories. Changes of lifestyle cannot be accomplished by short, temporary, interventions [71]. We cannot declare which effect the MoVo process models have regarding adherence rate and attrition rate in LTC elderly. But the MoVo model seems to have a positive effect on the adherence rate in the LTC elderly in our main study.

\subsection{Follow-up}

The impact of a treatment on public health is determined by the following factors: 1) the degree of benefit of the exercise (i.e. fewer falls, improving strength and balance) and 2) the changes in physical performance regarding independency and function. To evaluate and implement these aspects into public health programs, long-term follow-up studies should be implemented. The study presented in Chapter 7 shows evidence for improvement of the characteristics under evaluation but a follow-up study was not carried out.

Up to date, there are no recommendations regarding adequate follow-up length to evaluate the benefits of exercise and changes in physical performance regarding independency and functional status in elderly persons. Previous long-term follow-up studies measured six months [72, 73], twelve months [74] and seven years [75] after randomization. Future studies should take a long-term follow-up study design to provide health promotion in the context of Public Health of at least six months. 


\subsection{Training regimes}

Rate of force development (RFD) is important for movements of short duration, for example to prevent an imminent fall [76], and it is linked to power as it determines the magnitude of acceleration in the initial phase of a movement, thereby influencing movement velocity. From a functional view, RFD is needed for daily motor activities (e.g. chair rising and stair climbing).

We have taken these aspects into account and investigated the strength components that are relevant to addressing them in daily life. Despite the implementation of the three feasibility studies (Chapters 4 to 6), no clear-cut normative suggestions for optimal vibration parameters or exercise prescription could be derived.

WBV affects neural drive while conventional strength training influences neural drive and muscle hypertrophy. It is known that a strength improvement is caused as a result of improved neural drive and muscle hypertrophy [32], but the dose-response relation is not explained conclusively [77]. Sherrington et al. [78] recommended strength training at least two times a week. Most studies included in two systematic reviews and the meta-analysis (Chapters 2 and 3) a training frequency of three sessions per week. For reasons of plausibility, the same training frequency was used in the studies included in this Doctoral Thesis (Chapters 4 to 7 ).

\section{Future research}

\section{Future research is required in the field of skilling-up training in long-term-care elderly.}

First, intervention studies should be performed on the basis of the functional classification GoGo, Slow-Go and No-Go in an elderly population [3]. However, a further sub-classification for the No-Go group should be used, for, when classifying No-Go elderly into mobile, wheelchair and bedridden we recommend using the basic mobility scale and walking scale to evaluate the functional performance level in elderly individuals according to Okochi et al. [79]. The items for basic mobility are 1. change lying position, 2. maintain sitting position without assistance, 3. transfer sit-to-stand and 4. maintaining standing position without help for 3 minutes. Each item is to be rated as "yes" or "no". The walking items are 1. moving around with a walking aid, 2. walking without assistance, 3. climbing up more than five stair-steps and 4. going out using public transportation. Go-Go and Slow-Go could do all of these. No-Go elderly who are mobile could perform basic mobility scale items and could not do the walking scale items. Wheelchair elderly could perhaps not stand for over three minutes but could move around. Bedridden elderly could be classified on the basic mobility scale. For mobile LTC and LTC elderly that are wheelchair bound, the Short Physical Performance Scale (SPPB) [59] could be used as an outcome parameter.

Second, the motivational aspect should be taken into account. To recruit sufficient volunteers, personal motivational interviews should be conducted with every eligible participant. Furthermore, 
to encourage adherence to this innovative skilling-up program, we suggest that participants are met and returned to their rooms by support personnel. This personal contact provides the possibility for active promotion of a positive attitude towards exercise during the intervention period.

Third, the sample size should be increased for future studies. In practice, this study showed that it is possible to recruit enough LTC elderly in need of care who live in a nursing home for treatment or sensimotor training. But in order to obtain significant evidence of an effect after SRWBV and VG intervention, studies with larger sample sizes are needed.

Fourth, despite growing evidence supporting WBV training in the last ten years, an optimal training regime is still unclear. There is a need for future research to assess the influence of SR-WBV and VG on functional performance in LTC elderly in need of care if they are mobile. Any future research should be based on the findings of this thesis, namely: 1. The minimum number of training sessions is not to be less than three times a week. 2. Training parameters such as intensity (e.g. vibration frequency, VG music rhythm: beats per minute), training duration (increasing exercise duration), study period (> eight weeks with follow up) and rest between sets (decreasing pause duration) should be designed progressively.

Fifth, a new research field is arising for LTC elderly in need of care if they are not mobile (wheelchair bound) or they are bedridden. SR-WBV and sinusoidal WBV have the potential to improve the neuromuscular system $[14,15]$ and bone density [80]. With this potential for WBV as skilling-up training, future research is required to evaluate the variables feasibility, safety and effectiveness in LTC not mobile elderly and bedridden elderly.

Sixth, research is required to conduct follow-up effects of SR-WBV and VG on functional performance and strength. Follow-up studies will allow a more detailed investigation into public health in order to show how this innovative program could best serve health promotion.

Seventh, it is known that VG has a beneficial impact on cognition. Future research should be conducted to examine the effects of SR-WBV and VG on cognition in LTC mobile elderly in need of care.

\section{Implications for clinical practice}

In clinical practice, for those who want to start with exercise programs for elderly in need of care in the field of long-term care, we recommend proceeding as follows: 1) Evaluation of the current physical performance level, 2) training program in elderly in need of care in the field of long-term care, and 3) applying a motivational model for consolidating adherence and reducing attrition.

1) Evaluation of the current physical performance level should consider using a screening tool to evaluate the current physical fitness level. The aim is to perform individually adapted exercise sessions. We recommended the classification Go-Go, Slow-Go and No-Go. 
2) A training program for mobile elderly in need of care in the field of long-term care should include a combination of stochastic resonance whole-body vibration and video gaming in order to improve the physical performance level. The results of this thesis suggest that this innovative training program improves the neuromuscular system in such a manner that, after eight weeks performance is raised from a poor state to a moderate state. Consequently, we recommended that this innovative training program should embedded as skilling-up exercise in No-Go elderly in need of care in the field of long-term care to enhance physical performance and independence.

3) Applying a motivational model to improve adherence and reduce attrition. To maintain the adherence rate and prevent attrition during the training program we recommended compliance strategies in conjunction with the treatment. The compliance strategies should include information about the exercise and feedback about the training progress, motivation and support-staff accompaniment (e.g. pick-up to training session) during the training process.

\section{Overall conclusion}

Maintaining function and independence and reducing care is one of the primary objectives of any intervention in No-Go elderly in the field of LTC. We developed an innovative training program for skilling-up in LTC in need of care elderly who live in a nursing home. Our findings point to a well-developed, tailor made, and coordinated training program for skilling-up in No-Go elderly in the field of LTC in need of care who live in nursing homes. The research results of this thesis have built up knowledge about how to exercise as skilling-up in the field of LTC in need of care elderly, recommended how to apply a training regime into clinical practice and suggested which topics should be investigated in future research. Our developed innovative training program shows the potential benefit for skilling-up in LTC in need of care elderly. More specifically, two systematic reviews and meta-analyses and five studies were undertaken. Both systematic reviews and metaanalyses (Chapters 2 and 3) were able to illustrate a positive impact on balance and strength after WBV intervention. Furthermore, the first feasibility studies we conducted in Chapters 4, 5 and 6 of this thesis proved to be feasible and safe for Slow-Go and No-Go elderly after SR-WBV intervention. Our innovative training program study (Chapter 7) shows that LTC elderly in need of care can benefit from a combination of SR-WBV and VG intervention to increase physical performance and strength. The validity study in chapter 8 indicates only cadence as a valid spatio-temporal gait parameter for the RehaWatch ${ }^{\circledR}$ system. Based on the findings of this thesis we recommend SR-WBV and VG as a skilling-up exercise for LTC elderly in need of care. There should be a focus on the integration of the functional classification Go-Go, Slow-Go and No-Go and the sub-classification mobile, wheelchair and bedridden elderly into future research projects and clinical practice. In addition, the cognition of the elderly should be assessed and follow-up studies with larger sample sizes should to be carried out to assess whether these adaptations will result in greater effectiveness and efficiency. 


\section{References}

1. Chandler JM, Duncan PW, Kochersberger G, Studenski S: Is lower extremity strength gain associated with improvement in physical performance and disability in frail, community-dwelling elders? Arch Phys Med Rehabil 1998, 79(1):24-30.

2. Taaffe DR, Marcus R: Musculoskeletal health and the older adult. J Rehabil Res Dev 2000, 37(2):245-254.

3. Zeyfang A, Braun A: [Guidelines "Diabetes mellitus in the elderly"]. MMW Fortschritte der Medizin 2009, 151(20):33-35, 37.

4. Ferrucci L, Guralnik JM, Studenski S, Fried LP, Cutler GB, Jr., Walston JD, Interventions on Frailty Working G: Designing randomized, controlled trials aimed at preventing or delaying functional decline and disability in frail, older persons: a consensus report. J Am Geriatr Soc 2004, 52(4):625-634

5. Rogan S, Hilfiker R: [Training methods - increase muscle strength due to wholebody vibration - force with $\mathrm{Hz}$ ]. Sportverletz Sportschaden 2012, 26(4):185-187.

6. Herren K, Rogan S, Hilfiker R, Radlinger L: Vibrationen mit therapeutischen Effekten. PhysioActive 2009:39-44

7. Herren K, Rogan S, Radlinger L: Ganzkörpervibration als Krafttrainingsmethode. . Physioactive 2012, 5:23-29

8. Lam FM, Lau RW, Chung RC, Pang MY: The effect of whole body vibration on balance, mobility and falls in older adults: a systematic review and meta-analysis. Maturitas 2012, 72(3):206-213.

9. Sitja-Rabert M, Rigau D, Fort Vanmeerghaeghe A, Romero-Rodriguez
D, Bonastre Subirana M, Bonfill X: Efficacy of whole body vibration exercise in older people: a systematic review. Disabil Rehabil 2012, 34(11):883-893

10. Lau RW, Liao LR, Yu F, Teo T, Chung RC, Pang MY: The effects of whole body vibration therapy on bone mineral density and leg muscle strength in older adults: a systematic review and meta-analysis. Clin Rehabil 2011, 25(11):975-988.

11. Osawa $Y$, Oguma $Y$, Ishii N: The effects of whole-body vibration on muscle strength and power: a meta-analysis. J Musculoskelet Neuronal Interact 2013, 13(3):380-390.

12. Moher D, Schulz KF, Altman DG: The CONSORT statement: revised recommendations for improving the quality of reports of parallel-group randomised trials. Lancet 2001, 357(9263):1191-1194.

13. Rauch F, Sievanen H, Boonen S, Cardinale M, Degens $H$, Felsenberg D, Roth J, Schoenau E, Verschueren S, Rittweger $\mathrm{J}$ et al: Reporting whole-body vibration intervention studies: recommendations of the International Society of Musculoskeletal and Neuronal Interactions. J Musculoskelet Neuronal Interact 2010, 10(3):193-198.

14. Sievanen H, Karinkanta S, Moisio-Vilenius P, Ripsaluoma J: Feasibility of whole-body vibration training in nursing home residents with low physical function: a pilot study. Aging Clin Exp Res 2014, 26(5):511-517.

15. Zhang L, Weng C, Liu M, Wang Q, Liu L, He Y: Effect of whole-body vibration exercise on mobility, balance ability and general health status in frail elderly patients: a pilot randomized controlled trial. Clin Rehabil 
2014, 28(1):59-68.

16. Schmidt JA, Gruman C, King MB, Wolfson LI: Attrition in an exercise intervention: a comparison of early and later dropouts. $J$ Am Geriatr Soc 2000, 48(8):952-960.

17. Jette $A M$, Lachman $M$, Giorgetti $M M$, Assmann SF, Harris BA, Levenson C, Wernick M, Krebs D: Exercise--it's never too late: the strong-for-life program. Am J Public Health 1999, 89(1):66-72.

18. Lord SR, Ward JA, Williams P, Strudwick M: The effect of a 12-month exercise trial on balance, strength, and falls in older women: a randomized controlled trial. J Am Geriatr Soc 1995, 43(11):1198-1206.

19. Fairhall N, Langron C, Sherrington C, Lord SR, Kurrle SE, Lockwood K, Monaghan N, Aggar C, Gill L, Cameron ID: Treating frailty-a practical guide. BMC Med 2011, 9:83.

20. Skelton DA, Dinan SM: Exercise for falls management: Rationale for an exercise programme aimed at reducing postural instability. Physiotherapy Theory and Practice 1999, 15(2):105-120.

21. Kawanabe K, Kawashima A, Sashimoto I, Takeda T, Sato Y, Iwamoto J: Effect of whole-body vibration exercise and muscle strengthening, balance, and walking exercises on walking ability in the elderly. Keio J Med 2007, 56(1):28-33.

22. Pichierri G, Wolf P, Murer K, de Bruin ED: Cognitive and cognitive-motor interventions affecting physical functioning: a systematic review. BMC Geriatr 2011, 11:29.

23. Pichierri G, Coppe A, Lorenzetti S, Murer K, de Bruin ED: The effect of a cognitive-motor intervention on voluntary step execution under single and dual task conditions in older adults: a randomized controlled pilot study. Clin Interv Aging 2012, 7:175-184.

24. Pichierri G, Murer $\mathrm{K}$, de Bruin ED: $A$ cognitive-motor intervention using a dance video game to enhance foot placement accuracy and gait under dual task conditions in older adults: a randomized controlled trial. BMC Geriatr 2012, 12:74.

25. Schoene D, Valenzuela T, Lord SR, de Bruin ED: The effect of interactive cognitive-motor training in reducing fall risk in older people: a systematic review. BMC Geriatr 2014, 14:107.

26. van het Reve E, de Bruin ED: Strengthbalance supplemented with computerized cognitive training to improve dual task gait and divided attention in older adults: a multicenter randomized-controlled trial. BMC Geriatr 2014, 14:134.

27. van Het Reve E, Silveira P, Daniel F, Casati F, de Bruin ED: Tablet-based strengthbalance training to motivate and improve adherence to exercise in independently living older people: part 2 of a phase II preclinical exploratory trial. J Med Internet Res 2014, 16(6):e159.

28. Clark BC, Manini TM: Sarcopenia $=/=$ dynapenia. J Gerontol A Biol Sci Med Sci 2008, 63(8):829-834.

29. Clark BC, Manini TM: Functional consequences of sarcopenia and dynapenia in the elderly. Curr Opin Clin Nutr Metab Care 2010, 13(3):271-276.

30. Clark BC, Issac LC, Lane JL, Damron LA, Hoffman RL: Neuromuscular plasticity during and following $3 \mathrm{wk}$ of human forearm cast immobilization. J App/ Physiol (1985) 2008, 105(3):868-878.

31. Aagaard P, Simonsen EB, Andersen JL, Magnusson P, Dyhre-Poulsen P: Neural adaptation to resistance training: changes 
in evoked V-wave and H-reflex responses. J Appl Physiol (1985) 2002, 92(6):2309-2318.

32. Sale DG: Neural adaptation to resistance training. Medicine and science in sports and exercise 1988, 20(5 Suppl):S135-145.

33. Schmidtbleicher D, Haralambie G: Changes in contractile properties of muscle after strength training in man. European journal of applied physiology and occupational physiology 1981, 46(3):221-228.

34. Gruber M, Gollhofer A: Impact of sensorimotor training on the rate of force development and neural activation. Eur J Appl Physiol 2004, 92(1-2):98-105.

35. Beurskens R, Gollhofer A, Muehlbauer T, Cardinale M, Granacher U: Effects of heavyresistance strength and balance training on unilateral and bilateral leg strength performance in old adults. PLoS One 2015, 10(2):e0118535.

36. Granacher U, Gollhofer A, Kriemler S: Effects of balance training on postural sway, leg extensor strength, and jumping height in adolescents. Res Q Exerc Sport 2010, 81(3):245-251.

37. Granacher U, Gollhofer A, Strass D: Training induced adaptations in characteristics of postural reflexes in elderly men. Gait Posture 2006, 24(4):459-466.

38. Gruber M, Gruber SB, Taube W, Schubert M, Beck SC, Gollhofer A: Differential effects of ballistic versus sensorimotor training on rate of force development and neural activation in humans. J Strength Cond Res 2007, 21(1):274-282.

39. Kessler J, Radlinger L, Baur H, Rogan S: Effect of stochastic resonance whole body vibration on functional performance in the frail elderly: A pilot study. Arch Gerontol Geriatr 2014, 59(2):305-311.
40. Kramer A, Dettmers C, Gruber M: Exergaming with additional postural demands improves balance and gait in patients with multiple sclerosis as much as conventional balance training and leads to high adherence to home-based balance training. Arch Phys Med Rehabil 2014, 95(10):1803-1809.

41. de Bruin ED, Schoene D, Pichierri G, Smith ST: Use of virtual reality technique for the training of motor control in the elderly. Some theoretical considerations. Z Gerontol Geriatr 2010, 43(4):229-234 .

42. Derlin S, Boehme B, Leistritz L, Smolesnki UC: Validitätsuntersuchung zum neuen, innovativen Ganganalysesystem RehaWatch von Hasomed. Manuelle Medizin 2010, 48:254-259.

43. Schwenk M, Howe C, Saleh A, Mohler J, Grewal G, Armstrong D, Najafi B: Frailty and technology: a systematic review of gait analysis in those with frailty. Gerontology 2014, 60(1):79-89.

44. Schwesig R, Leuchte S, Fischer D, Ullmann $R$, Kluttig A: Inertial sensor based reference gait data for healthy subjects. Gait Posture 2011, 33(4):673-678.

45. Dixon-Woods $M$, Sutton $A$, Shaw R, Miller T, Smith J, Young B, Bonas S, Booth A, Jones $\mathrm{D}$ : Appraising qualitative research for inclusion in systematic reviews: a quantitative and qualitative comparison of three methods. J Health Serv Res Policy 2007, 12(1):42-47.

46. Lundh A, Gotzsche PC: Recommendations by Cochrane Review Groups for assessment of the risk of bias in studies. BMC Med Res Methodol 2008, 8:22.

47. Hartling L, Hamm M, Milne A, Vandermeer B, Santaguida PL, Ansari M, Tsertsvadze 
A, Hempel S, Shekelle P, Dryden DM. In: Validity and Inter-Rater Reliability Testing of Quality Assessment Instruments. Rockville (MD); 2012.

48. Schmidtbleicher D, Turbanski S, Haas CT: Effects of whole-body vibration on postural control in Parkinson's disease. Mov Disord 2004, 19:S185-S185.

49. Haas CT, Schmidtbleicher D: Potential of Stochastic Resonance in Neurorehabilitation. Isokinetic and Exercise Science 2006, 14(2):144-146.

50. Haas CT, Schmidtbleicher D: Zu den Effekten mechanischer Schwingungsreize bei Morbus Parkinson. Rheuma Aktuell 2002, 3:8-10.

51. Haas CT, Schmidtbleicher D: About Effects of Exercise and Stochastic Resonance on Neuroplasticity and Neuroprotection. In: Current Results of Strength Training Research Edited by Fröhlich M, Giessing, J., vol. 2. Göttingen: Cuviliier Verlag; 2007: 105-118.

52. Haas CT, Turbanski S, Schmidtbleicher D: Präventive und rehabilitative Aspekte im alpinen Skirennlauf. In: Österreichische Sportärzte Woche; Zell am See. 2004.

53. Haas CT, Turbanski S, Schmidtbleicher D: Wie gezielte Unordnung im Training für gezielte Ordnung im Training sorgt. In: Forschung Frankfurt. vol. 4: Universität Frankfurt; 2006: 19-24.

54. Haas CT, Turbanski S, Kaiser I, Schmidtbleicher D: [Biomechanical and physiological effects of oscillating mechanical stimuli in humans]. Deutsche Zeitschrift für Sportmedizin 2004, 55(2):3443.

55. Haas CT, Turbanski S, Schmidtbleicher D: Vibrationstraining in der Rehabilitation von Gang- und Gleichgewichtsstörungen. Focus Neurogeriatrie 2007, 1(3):18-19.

56. Kaptchuk TJ: Effect of interpretive bias on research evidence. BMJ 2003 326(7404):1453-1455.

57. Kane MT: The assessment of professional competence. Eval Health Prof 1992, 15(2):163-182

58. Knickman JR, Snell EK: The 2030 problem: caring for aging baby boomers. Health Sen Res 2002, 37(4):849-884.

59. Guralnik JM, Simonsick EM, Ferrucci L, Glynn RJ, Berkman LF, Blazer DG, Scherr PA, Wallace RB: A short physical performance battery assessing lower extremity function: association with self-reported disability and prediction of mortality and nursing home admission. Journal of gerontology 1994, 49(2):M85-94.

60. Organization WH: Global recommendations on physical activity for health. In. Gemeva, Switzerland; 2010.

61. Craig R, Mindell J: Health survey for England. London: Social Care Information Centre; 2012.

62. 412 SE: Sport amd phyiscal activity. In. Wave EB80.2; 2014

63. Ryan $R$, Williams G, Patrick $H$, Deci E: Self-determination theory and physical activity: The dynamics of motivation in development and wellness. Hellenic Journal of Psychology 2009, 6:107-124.

64. Potempa KM: The Healthy Ageing Model: Health behaviour change for older adults Collegian 2010, 17:51-55.

65. Prochaska JO, DiClemente CC, Norcross JC: In search of how people change. Applications to addictive behaviors. Am Psychol 1992, 47(9):1102-1114.

66. Bandura A: Health promotion from the 
perspective of Social Cognitove Theory. In: Understanding and changing health behaviour: From health beliefs to selfregulation. Edited by Norman P. AC, Conner M. . Amsterdam, NL: Harwood Acadenic Publishers; 2000: 299-339.

67. Ajzen I: The Theory of Planned Behavior. Organ Behav Hum Dec 1991, 50(2):179211.

68. Gollwitzer PM: Implementation intentions - Strong effects of simple plans. American Psychologist 1999, 54(7):493-503.

69. Göhner W, Fuchs R: Änderung des Gesundheitsverhaltens. Göttingen, Germany: Hogrefe; 2007.

70. Fuchs R: [MoVo model as theoretical framework for health behaviour change]. In: [Setting up a physically active lifestyle]. Edited by Fuchs R GW, Seelig H. Göttingen, Germany: Hogrefe; 2007: 317-325.

71. Biddle SJH, Fuchs R: Exercise psychology: A review from Europe. Psychology of Sports and Exercise 2009, 10:410-419.

72. Sitja-Rabert M, Martinez-Zapata MJ, Fort Vanmeerhaeghe A, Rey Abella F, RomeroRodriguez D, Bonfill X: Effects of a whole body vibration (WBV) exercise intervention for institutionalized older people: a randomized, multicentre, parallel, clinical trial. J Am Med Dir Assoc 2015, 16(2):125131.

73. Sitja-Rabert M, Martinez-Zapata MJ, FortVanmeerhaeghe A, Rey-Abella F, RomeroRodriguez D, Bonfill X: Whole body vibration for older persons: an open randomized, multicentre, parallel, clinical trial. BMC Geriatr 2011, 11:89.

74. Kennis E, Verschueren SM, Bogaerts A, Coudyzer W, Boonen S, Delecluse C: Effects of fitness and vibration training on muscle quality: a 1-year postintervention follow-up in older men. Arch Phys Med Rehabil 2013, 94(5):910-918.

75. Kennis E, Verschueren SM, Bogaerts A, Van Roie E, Boonen S, Delecluse C: Longterm impact of strength training on muscle strength characteristics in older adults. Arch Phys Med Rehabil 2013, 94(11):2054-2060.

76. Caserotti $P$ : Strength training in older adults: changes in mechanical muscle function and functional performance. The open sports sciences journal 2010, 3:62-66.

77. Aagaard P, Suetta C, Caserotti P, Magnusson SP, Kjaer M: Role of the nervous system in sarcopenia and muscle atrophy with aging: strength training as a countermeasure. Scand J Med Sci Sports 2010, 20(1):49-64.

78. Sherrington C, Whitney JC, Lord SR, Herbert RD, Cumming RG, Close JC: Effective exercise for the prevention of falls: a systematic review and meta-analysis. J Am Geriatr Soc 2008, 56(12):2234-2243.

79. Okochi J, Takahashi T, Takamuku K, Escorpizo R: Staging of mobility, transfer and walking functions of elderly persons based on the codes of the International Classification of Functioning, Disability and Health. BMC Geriatr 2013, 13:16.

80. Calendo LR, Taeymans J, Rogan S: [Does muscle activation during whole-body vibration induce bone density improvement in postmenopausal women? [A systematic review]. Sportverletz Sportschaden 2014, 28(3):125-131. 



\title{
Chapter 10
}

\author{
Valorization
}




\section{Relevance}

This dissertation adds new knowledge to the field of training regimes in No-Go elderly individuals. The research for this thesis used a whole-body vibration device that produces stochastic resonance vibration and an exergame device in the form of a dance-game. This Doctoral Thesis also revealed that whole-body vibrations are a safe training method for Go-Goes elderly individuals (Chapter 4 and 5) and for No-Goes elderly individuals (Chapter 6). This work also showed that the use of whole-body vibration and exergaming as skilling-up training regime to improve balance, strength and gait in especially No-Go elderly individuals may be effective (Chapter 7). Based on the results of this thesis, the following conclusions can be made: firstly, SR-WBV demonstrate no side-effects and considered not be harmful under training conditions of use in this dissertation. Secondly, there is strong evidence that an implementation of this innovative training program into clinical practice may be of advantage for the elderly, especially for the No-Go elderly. From these cognitions, further research should be carried out to strengthen the findings of this thesis and to evaluate cost-effectiveness (and cost-utility) as well as, costbenefit of this new training intervention.

This chapter explains how to translate the findings of the doctoral thesis into a target group, it exemplifies why this training regime can be considered as an innovative program; it describes in which services this training regimes can be transferred and it demonstrates what the next research steps are to be made.

\section{Target group}

The findings of this Doctoral thesis are relevant for elderly individuals. It is known that aging is an individual process and that the human body has two ages. Chronological age is the current lifetime in years, while biological age is the current state of physical capacity and cognitive ability of an individual. Biological age should be taken into account in the context of this present work, because in elderly decreased physical capacity and cognitive ability may impair their health [1]. This Doctoral Thesis focused on No-Goes elderly individuals. Based on the findings, future studies could further develop the study methodology with a prolongation of the intervention period to evaluate the effects on physical performance. Furthermore, the cognition level and the cost-effectiveness (and cost-utility) as well as, cost-benefit of this new training intervention should be examined.

The findings are relevant for all physicians and health professionals who are working with NoGoes elderly individuals. Physicians and health professionals could encourage No-Go elderly individuals to carry out exercise programs to improve their physical performance. 


\section{Innovative product}

Daily activities such as washing, dressing, going to the restroom, moving, standing up and eating are important motor actions that an elderly individual has to carry out to remain independent. However, many elderlies are in need for care. In Switzerland, 4\% of elderly people over 65 living at home are not able to carry out one of these daily activities while in nursing homes, the figure rises to $67 \%$ [2]. Over $37 \%$ of the elderly are not able to carry out a minimum of five activities of daily living [2]. In this case every person should have access to safe and effective therapy. The public health system in western societies often faces major challenges to improve efficiency in medical care and organizational age-appropriate conditions. This thesis presents evidence for the implementation of whole-body vibration and exergame as a new and innovative training method for elderly in need of care (i.e. No-Go elderly) (Chapter 9). This innovative training method can be seen as a component of a modular system concept. This is based on that elderly individual's biological age appears in different forms [3]. The concept includes examination of the elderly person classification into Go-Go, Slow-Go and No-Go elderly and the whole-body vibration and exergame treatment method of an elderly individual. In this context, 'examination' means to evaluate the current physical and mental function of an elderly person. In addition, physical performance (e.g. balance, strength) should be assessed.

\section{Activities and product}

Based on that, the evidence based findings of this thesis is to integrate into a practical therapy or treatment concept to increase motor ability of elderly (No-Go) individuals. This innovative training program supports elderly individuals by means of a comprehensive package. This comprehensive package includes the examination of the physical and mental function level and a training regime that integrates a motivation-volition model. There is a bulk of evidence on the benefits of increased physical activity on health status (in the elderly). Despite the obvious advantages of physical activity, it is not easy to motivate (elderly) persons towards more exercise. It Is well known, if a person wants to achieve a goal (e.g. improvement of muscle strength) positive concepts to inspiring themselves and strive to attain their potential is required. Motivation is needed. This Doctoral-Thesis used a motivation-volitions model with success to maintain up the adherence-rate of the study participants. The training regimen in this Doctoral Thesis is based on the motivation-volition model [4]. However, the motivation-volition model was detailed reported in Chapter 9.

This thesis used the Short Physical Performance Battery Test (SPPB) to test the current physical performance level. The SPPB shows good validity and reliability in diverse populations [5, 6]. SPPB was used for all three groups (Go-Go, Slow-Go and No-Go). Additionally, specific measurements were used to evaluate strength or flexibility of individual muscles.

Another recommendation is the De Morton Mobility Index (DEMMI). The DEMMI was used for all elderly acute medical patients [7], for community-dwelling adults who require informed care 
[8] and for healthy community-dwelling elderly individuals [9]. The DEMMI is an interval-level unidimensional scale, which consists of 15 hierarchical items that estimate mobility across a spectrum from bed-bound to independent mobility [7]. The advantage of this assessment is that the No-Go group can then be subdivided into 1) mobile elderly who can move with aids, b) elderly individuals who cannot move by themselves and are dependent on a wheelchair, or c) elderly individuals who are bedridden. Further studies should use the DEMMI for the No-Gogroup.

To evaluate the mental function of an elderly individual the Mini-Mental State Examination (MMSE) can be proposed. The MMSE provides a brief screening method assessing the severity of cognitive impairment and documenting cognitive changes occurring over a time period [10]. This doctoral thesis used the MMSE as an instrument for inclusion and exclusion criterion.

\section{Next steps}

The results of this Thesis suggest that not all the elderly may equally benefit from this innovative method to improve motor ability. Especially the No-Go groups showed higher effect-sizes (Chapter 3: meta-analysis, Chapter 4: SR-WBV shows no effect in Slow-Goes, Chapter 7: SRWBV and exergaming shows beneficial effects in No-Goes). Therefore, it can be proposed as "best practice" that researchers and clinicians may classify their participants and patients into Go-Go, Slow-Go and No-Go when working with elderly persons to apply the best training for the specific group.

This thesis does not provide information about monetary costs and benefits of this innovative method in elderly individuals. Future health economic evaluations studies should focus on the cost-effectiveness, cost-utility and cost-benefit of this WBV and exergaming program. The results of such health-economic evaluations can give new insight into the precise cost of this training regime and allow comparison among alternative training programs. These results may be of uppermost relevance for elderly individuals, insurers, government and society in general. At present, this Thesis evaluated side-effects and could present that SR-WBV is a safe training method in Slow-Go and No-Go elderly individuals. Further findings are an improved physical performance level (e.g. strength) in No-Go elderly individuals. These results can be used by insurers, government and society as background information to develop new training regimes for No-Go elderly in Switzerland.

The classification of functionality in Go-Go, Slow-Go and No-Go elderly allows to better prescribe and develop individually adapted training programs. Go-Go and Slow-Go elderly may profit more from traditionally exercise while No-Go elderly may yield highest benefits by a training regimen consisting of whole-body vibration and exergaming. 
Moreover, the findings of this thesis are especially relevant for the members of the No-Go group. This innovative program shows that especially the No-Go elderly may profit from WBV and exergaming to improve balance, strength and gait (Chapter 7). It can be argued that this innovative training program could also be used as a preventive measure against dynapenia, because WBV and exergame is a sensorimotor training method that stimulates the nervemuscle-system [11]. Moreover, this valid intervention method increases muscle strength in elderly individuals [12]. 


\section{References}

1. Rogan S, Radlinger L: From No-Go to GoGo. Future trainung procedures for elderly. J Gerontol Geriatr Res 2016, 5:278.

2. Statistik Bf: Gesundheit von Betagten in Alters- und Pflegeheimen. In. Edited by EDI EDfl. Neuchatel: Bundesamt für Statistik; 2012.

3. Rogan S: Innovative training program for elderly in long-term care. Journal of Health Care: Current Reviews 2015, 2:40.

4. Fuchs R: [MoVo model as theoretical framework for health behaviour change]. In: [Setting up a physically active lifestyle]. Edited by Fuchs R GW, Seelig H. Göttingen, Germany: Hogrefe; 2007: 317-325.

5. Freire AN, Guerra RO, Alvarado B, Guralnik JM, Zunzunegui MV: Validity and reliability of the short physical performance battery in two diverse older adult populations in Quebec and Brazil. J Aging Health 2012, 24(5):863-878.

6. Volpato S, Cavalieri M, Sioulis F, Guerra G, Maraldi C, Zuliani G, Fellin R, Guralnik JM: Predictive value of the Short Physical Performance Battery following hospitalization in older patients. J Gerontol A Biol Sci Med Sci 2011, 66(1):89-96.

7. de Morton NA, Davidson M, Keating JL: The de Morton Mobility Index (DEMMI): an essential health index for an ageing world. Health Qual Life Outcomes 2008, 6:63.

8. de Morton NA, Meyer C, Moore KJ, Dow $\mathrm{B}$, Jones $\mathrm{C}$, Hill $\mathrm{K}$ : Validation of the de Morton Mobility Index (DEMMI) with older community care recipients. . Australasian journal on ageing, 2011, 30(4):220-225.

9. Davenport SJ, de Morton NA: Clinimetric properties of the de Morton Mobility Index in healthy, community-dwelling older adults. Arch Phys Med Rehabil 2011, 92(1):51-58.

10. Tombaugh TN, Mclntyre NJ: The minimental state examination: a comprehensive review. J Am Geriatr Soc 1992, 40(9):922935.

11. Rogan S, Hilfiker R, Schenk A, Vogler A, Taeymans J: Effects of whole-body vibration with stochastic resonance on balance in persons with balance disability and falls history - a systematic review. Res Sports Med 2014, 22(3):294-313.

12. Rogan S, Schmidtbleicher D, Radlinger L: Immediate effects after stochastic resonance whole-body vibration on physical performance on frail elderly for skilling-up training: a blind cross-over randomised pilot study. Aging Clin Exp Res 2014, 26(5):519-527. 



\section{Chapter 11}

Summary / Zusammenfassung 


\section{Summary}

This thesis examines the implementation of training options in the field of long-term care (LTC) for elderly in need of care. New and innovative training programs consisting of whole body vibration with stochastic resonance (SR-WBV) and exergaming / video gaming (VG) are in focus to verify how these training regimes may be used in the field of LTC for elderly in need of care. To verify this innovative training program, the following steps were conducted: systematic gathering of information regarding the effects of whole body vibration (WBV) from scientific publications to acquire empirical knowledge, from which evidence is generated. In order to verify the validity of these findings, at the beginning a feasibility study with Go-Go and Slow-Go participants was conducted. Subsequently a feasibility study with No-Go elderly was carried out. Then the main study was conducted, wherein the effects after SR-WBV and VG were studied in No-Go elderly. Finally a validity study was carried out, where the RehaWatch ${ }^{\circ}$ system was compared with the GAITRite ${ }^{\circledR}$ system.

Chapter one provides the basics of the topic of this thesis. It examines life expectancy at age 65, the aging process and the associated loss of function, the classification of functioning Go-Go, Slow-Go and No-Go, and the theoretical approach of SR-WBV and VG as a training regime, the objective of this thesis.

The continuous increase in average life expectancy at the age of 65 in Europe has a discrepancy observed in health life expectancy at the same age. Currently, the population in Europe lives independently for almost 8.6 years of the reported average life expectancy at age 65 of 18.9 years. The other half of the average number of years of life expectancy at the age of 65 depends on a second person providing care. Our endeavor should no longer be simply to extend lifespan, but rather to extend health span.

Aging processes are associated with physical changes (strength and balance loss) and cognition changes. These changes manifest themselves in loss of function. Aging is a measure of the function at a given time. For this reason it is necessary to determine the current functional status of an elderly person. Because aging processes vary from case to case, it is appropriate to divide the elderly according to their current functional status and to carry out a specific training program. The classification of functional status carried out in Go-Go (independent living elderly individuals), Slow-Go (needy elderly individuals) and No-Go (LTC elderly in need of care, helpless living). For Go-Go and Slow-Go, traditional training programs are used. However, for No-Go, training regimes need to be structured such that they have a low amount of load, which serves as skilling-up. In this case, innovative training programs such as SR-WBV and VG provide an opportunity.

This thesis follows the hypothesis that SR-WBV and VG affect the physical performance and function positively in No-Go elderly in the field of LTC of elderly in need of care. For this reason, new findings should be generated by scientific research. 
Chapter two includes a systematic review and meta-analysis of effects after WBV training on static, dynamic and functional balance in elderly individuals. In this study, 15 articles were included and were analyzed in terms of bias, homogeneity / heterogeneity and intervention effects. These studies showed at least a control group design and conducted a baseline measurement and a post-intervention measurement. The meta-analysis calculated the effect size of WBV versus control group on static, dynamic and functional balance from each included study. The results illustrate a high risk of bias. Most studies exhibit a high risk of bias on "allocation concealment", "blinding" and "incomplete outcome data". In addition, heterogeneity of the studies will be added, which prevent a clear statement in terms of effects on static, dynamic and functional balance. Despite the limitations, this meta-analysis provides useful information on the effects of WBV training for improving balance in older people. Furthermore, with a high level of evidence, this work supplies some information on the training protocols and the defined endpoints that should be considered in future studies in order to demonstrate the impact on balance..

In contrast, the described systematic review and meta-analysis in chapter three is more able to draw conclusions in terms of effects after WBV training on maximum voluntary contraction, dynamic strength, power, rate of force development and functional strength. 37 articles were included that had a control group design and measured outcome variable before and after the intervention period. For each study, the effect size was calculated for muscle strength (WBV group versus control group and WBV group versus strength training group) in Go-Go, SlowGo and No-Go. The results suggest that due to WBV training, muscle strength improved in the No-Go group towards Slow-Go and Go-Go. The results indicate that WBV can be used as a skilling-up exercise in elderly individuals, who are not able to perform traditional strength training. This review shows a moderate bias rate and low heterogeneity.

Chapter four is a feasibility study on a single blinded randomized controlled trial after a single SR-WBV intervention session in No-Go and Slow-Go elderly. The participants in the intervention group $(n=10)$ carried out SR-WBV training on the Zeptor ${ }^{\circledR}$ Med. For this purpose the participants stood with feet shoulder-width apart, with slightly flexed hip and knee joints and with shoes on two foot plates, five times over one minute with an intensity of $5 \mathrm{~Hz}$, Noise 4. The control group received no intervention and sat on a chair for ten minutes. The primary outcome was the "Criteria of Success", including the recruitment rate, the adherence rate and the attrition rate. Secondary outcome measures were: semi tandem stand test, Functional Reach Test, Expanded Timed Get Up and Go test, single task, dual task, hand reaction time and foot reaction time. The feasibility of this study was confirmed. SR-WBV is safe to use for Go-Go and Slow-Go and it was well tolerated, showing beneficial effects on balance.

Chapter five describes a feasibility study with SR-WBV with a randomized controlled single blinded cross-over design, in Go-Go and Slow-Go elderly. 20 participants were randomly assigned in either Group A (four weeks SR-WBV at $5 \mathrm{~Hz}$, Noise 4, followed by a 16-day wash- 
out period, then four weeks SR-WBV at $1 \mathrm{~Hz}$, Noise 1) or group B (four weeks SR -WBV at $1 \mathrm{~Hz}$, Noise 1, 16-day wash-out period, four weeks SR-WBV at $5 \mathrm{~Hz}$, Noise 4). The participants stood in shoes on two foot plates, with feet shoulder-width apart, and slightly flexed hip and knee joints. The primary endpoint was "Criteria of success" (recruitment rate, adherence rate and attrition rate) and safety. Secondary outcome measures were: semi-tandem-stand, Functional Reach Test, Expanded Timed Get-Up-and-Go test, single task, dual task, hand and foot reaction time. This feasibility study showed that the examination protocol could be used for future studies. SRWBV is certainly feasible for Go-Go and Slow Go volunteers. This feasibility study was able to include a sufficient number of elderly individuals (55\%) and presents an acceptable adherence rate of $85 \%$ and an attrition rate of $15 \%$. SR-WBV training at $5 \mathrm{~Hz}$, Noise 4 seems to be able to generate sufficient stimuli to improve the total time in the Expanded Timed Get Up and Go test.

Chapter six is a feasibility study in a double-blinded randomized controlled cross-over study with SR-WBV in No-Go elderly. Of 24 possible participants, 9 were included. They were randomly allocated in either Group A (four weeks SR-WBV at $6 \mathrm{~Hz}$, Noise 4, followed by a 16-day washout period, then four weeks SR-WBV at $1 \mathrm{~Hz}$, Noise 1) or group B (four weeks SR-WBV at $1 \mathrm{~Hz}$, Noise 1, 16-day wash-out period, four weeks SR-WBV at $6 \mathrm{~Hz}$, Noise 4). The participants stood in shoes on two foot plates, with feet shoulder-width apart, and slightly flexed hip and knee joints. One endpoint was the "Criteria of success (recruitment rate, adherence rate and attrition rate) and safety. Primary outcome: Expanded Timed Get-Up-and-Go test. Secondary outcome: ShortPhysical-Performance-Battery-Test, muscle strength and chair rise. This feasibility study showed that the study protocol might needs to be adjusted for future studies. At $56 \%$, the attrition rate was very high and the adherence rate of $81.6 \%$ was moderate. Effects were observed in all variables for SR-WBV intervention at $6 \mathrm{~Hz}$, Noise. 4.

In chapter seven, the results of a single blinded randomized controlled trial are described. This study examined the effects, after an eight-week training period, of SR-WBV and VG on physical performance and muscle strength in elderly in need of care in the field of LTC. An independent statistician carried out the grouping. 30 elderlies in need of care were divided into an intervention group (IG) or a sham group (SG). The IG completed the following intervention: eight weeks, 3x/ week, $5 \times 1$-minute vibration and 1-minute rest between sets. The SR-WBV started with a base frequency of $3 \mathrm{~Hz}$, Noise 5 and was progressively increased to $6 \mathrm{~Hz}$, Noise 5 . Initial position was a slight knee flexion, upright upper body, possible free-standing and without shoes on the foot plates. From week to week, the starting position was altered, from the parallel stand via step position to dynamic squats up to 90 degrees. From the fifth week, VG was added. The participants performed their VG on a metal dance plate with arrows. The VG display was projected onto a wall. Scrolling arrows moving upwards across the screen cued each move. The participants had the task to step in time to the music on the arrows on the plate when the arrows on the screen reached the fixed raster graphic at the top - sometimes right, sometimes down, sometimes to the left, etc. The rhythm got faster from song to song, increasing from 32 to 137 
beats per minute.

The following intervention was completed: over eight weeks the SG stood on the SR-WBV at 1 $\mathrm{Hz}$, Noise 1. From the fifth week a machine-controlled trampoline was used, which produced sinusoidal vibrations every second. To disable any effect on balance, the participants had to hold themselves steady on the railing.

The primary outcome was the Short Physical Performance Battery test. The secondary outcome was the isometric voluntary maximum contraction, isometric rate of force development of the thigh extensors and hamstrings. In addition, sub-values of isometric maximum voluntary contraction at 30,50, 100 and $200 \mathrm{~ms}$ and sub-values of isometric rate of force development in the range between $0-30,0-50,0-100$ and 100-200ms were determined. This study showed that a combination of SR-WBV and VG could significantly improve the outcome variables SPPB, subvalues of isometric voluntary contraction at $30,50,100$, and $200 \mathrm{~ms}$, the isometric rate of force development and sub-values of isometric rate of force development in the range between 0-30, 0-50, 0-100 and 100-200ms.

Chapter eight presents the results of a validity study. In this case the validity of an accelerometer, the RehaWatch ${ }^{\circledR}$ system, was compared with the GAITRite ${ }^{\circledR}$ system during single task (ST) and dual task (DT) conditions. The following gait parameter data were collected: standing duration time left and right leg, speed, cadence and stride left and right leg. This study included 23 participants, with an average age of 90.90 ( \pm 8.4) years. The participants performed four different measurements (normal walking during ST, normal walking during DT, fast walking during ST and fast walking during DT), over a walk distance of 13 meters. The conclusion of the study is that only the cadence values are valid and may be compared with other cadence values. Furthermore, this study demonstrated that No-Go participants are not able to walk at faster speed.

Chapter nine discusses the main results and the methodological considerations of this thesis. In addition, the limitations of this thesis are critically examined. From this, the conclusions were drawn to provide recommendations for future research and to provide practical instructions for an implementation of the innovative training program for No-Go elderly. 


\section{Zusammenfassung}

Diese Dissertation widmet sich der Implementierung von Trainingsmöglichkeiten im Bereich der Langzeitpflege für pflegebedürftige alte Menschen. Hierbei rücken neue innovative Trainingsprogramme bestehend aus Ganzkörpervibration mit stochastischer Resonanz (SRGKV) und Exergames in den Fokus. Es soll überprüft werden, inwiefern diese Methoden als Trainingsregime bei betagten pflegebedürftigen Menschen eingesetzt werden können. Dafür wurden folgende Arbeitsschritte durchgeführt: Systematisches sammeln von Informationen bezüglich Effekten von Ganzkörpervibration aus wissenschaftlichen Publikationen um empirisches Wissen zu erwerben und um daraus Erkenntnisse zu generieren. Um die Richtigkeit oder Falschheit dieser Erkenntnisse zu überprüfen, wurde zu Beginn eine Machbarkeitsstudie mit mobilen (Go-Go) und in der Mobilität leicht eingeschränkten (Slow-Go) Probanden durchgeführt. Folge der Machbarkeitsstudie war eine Pilotstudie mit pflegebedürftigen, in ihrer Mobilität stark eingeschränkten (No-Go) Probanden. Im Anschluss daran erfolgte die Hauptstudie, in welcher die Effekte nach SR-GKV und nach der Durchführung von Exergames bei No-Go Probanden untersucht wurde. Der Abschluss bildete eine Validitätsstudie, in welcher das RehaWatch ${ }^{\circledR}$ System mit dem GAITRite ${ }^{\circledR}$ System verglichen wurde.

Kapitel eins vermittelt die Grundlagen für das theoretische Themengebiet dieser Thesis. Es beleuchtet die Lebenserwartung ab dem 65. Lebensjahr, den Alterungsprozess und die damit verbundenen Funktionsverluste, die Klassifikation der Funktionsfähigkeit Go-Go, Slow-Go und No-Go, den theoretischen Aansatz von SR-GKV und Exergames als Trainingsregime und die Zielstellung dieser Thesis, Effekte nach SR-GKV und Exergames bei pflegebedürftig Betagten in der No-Go Gruppe hinsichtlich der körperlichen Leistungsfähigkeit zu untersuchen.

Der kontinuierliche Anstieg der durchschnittlichen Lebenserwartung ab dem 65. Lebensjahr in Europa steht in zunehmender Diskrepanz zur gesundheitlichen Lebenserwartung ab dem 65. Lebensjahr, die beobachtet werden. Momentan kann in Europa die Population nur knapp die Hälfte der durchschnittlich angegebenen Lebenswartung ab dem 65. Lebensjahr von 18.9 Jahren unabhängig und gesund leben. Die andere Hälfte der Lebenserwartung ab dem 65 . Lebensjahr ist sie abhängig von einer dritten Person. Unser Bestreben sollte nun darin liegen, nicht die Lebensspanne zu verlängern, sondern stattdessen die zu erwartenden gesunden Lebensjahre zu erhöhen.

Alterungsprozesse sind mit körperlichen und geistigen Veränderungen verbunden. Diese Veränderungen äussern sich in Funktionsverlust, zum Beispiel Kraft- und Gleichgewichtsverlust. Alterung stellt ein Mass für die Funktion zu einem gegebenen Zeitpunkt dar. Aus diesem Grund gilt es, den aktuellen Funktionszustand zu erheben. Da Alterungsprozesse individuell unterschiedlich ablaufen, ist es angebracht, ältere Personen nach ihrem aktuellen Funktionszustand einzuteilen, um ein gezieltes Training durchführen zu können. Die Einteilung des Funktionszustands erfolgt in Go-Go (unabhängig lebende), Slow-Go (hilfsbedürftige) und No-Go (pflegebedürftig, auf Hilfe 
angewiesene). Für Go-Go und Slow-Go kommen traditionelle Trainingsprogramme zum Einsatz. Für No-Go hingegen müssen Trainingsprogramme so aufgebaut sein, dass sie einen geringen Belastungsumfang aufweisen, die als Skilling-up dienen. Mit Skilling-up ist in diesem Kontext gemeint, die Zielpopulation auf traditionelle Trainingsprogramme hin zu trainieren. Hierzu bieten sich innovative Trainingsprogramme wie zum Beispiel SR-GKV und Exergames an. Aus diesem Grund wurde für die Studien jeweils Short-Physical-Performance-Battery-Test gewählt.

Diese Dissertation folgt der Hypothese, dass SR-GKV und Exergames die körperliche die funktionelle Leistungsfähigkeit für pflegebedürftige alte Menschen im Bereich der Langzeitpflege in der No-Go-Gruppe positiv beeinflusst. Aus diesem Grund sollten neu gewonnene Erkenntnisse durch wissenschaftliche Forschung generiert werden.

Kapitel zwei beinhaltet eine systematische Literaturübersichtsarbeit und Meta-Analyse von Trainingseffekten nach einem Ganzkörpervibrationstraining auf das statische, dynamische und funktionelle Gleichgewicht bei älteren Menschen. In diese Arbeit konnten 15 Studien eingeschlossen und hinsichtlich derer Verzerrung, Homogenität/Heterogenität und Effekte analysiert werden. Diese Studien wiesen mindestens ein Kontrollgruppendesign auf und führten eine Baseline- und Nachuntersuchungsmessung durch. Die Meta-Analyse (oder Metaanalyse) berechnete aus jeder Studie die Effektgrösse von GKV versus Kontrollgruppe auf das statische, das dynamische und auf das funktionelle Gleichgewicht. Die Ergebnisse veranschaulichen ein hohes Risiko für Verzerrung. Die meisten Studien weisen ein hohes Verzerrungsrisiko für „verborgene Zuweisung“, „Verblindung“ und „unvollständige Ergebnisdaten“ auf. Zudem kommt eine Heterogenität der Studien hinzu, die eine klare Aussage hinsichtlich Effekte auf das statische, dynamische und funktionelle Gleichgewicht verunmöglichen. Trotz der Limitierungen liefert die Metaanalyse nützliche Informationen über die Wirkung von GKV zur Gleichgewichtsverbesserung bei älteren Menschen. Des Weiteren bietet sie dieser Arbeit einige wertvolle Hinweise zu den Trainingsprotokollen und den definierten Endpunkten, die in zukünttigen Untersuchungen berücksichtigt werden sollten, um die Auswirkung auf das Gleichgewicht mit hohem Evidenzgrad zu belegen.

Im Gegensatz dazu vermag die im Kapitel drei beschriebene systematische Literaturübersichtsarbeit und Metaanalyse Aussagen hinsichtlich Effekte nach GKV hinsichtlich der Maximalkraft, der dynamischen Kraft, der Power, der Schnellkraft und der funktionellen Kraft eher eine Aussage treffen zu können. 37 Studien konnten eingeschlossen werden, die einerseits ein Kontrollgruppendesign, andererseits Vor- und Nachuntersuchungen aufwiesen. Für jede Studie wurde die Effektgösse für die Muskelkraft (GKV-Gruppe versus Kontrollgruppe und GKVGruppe versus Krafttrainingsgruppe) in den Gruppen Go-Go, Slow-Go und No-Go berechnet. Die Ergebnisse stellen dar, dass sich die Muskelkraft in der No-Go-Gruppe nach einem GKV-training gegenüber den beiden anderen Gruppen (Go-Go und Slow-Go) verbessert. Die Ergebnisse deuten an, dass GKV als Skilling-up-Training bei älteren Menschen, die noch nicht in Lage sind, 
traditionelles Krafttraining durchzuführen, effizient genutzt werden kann. Diese Übersichtsarbeit zeigt eine moderate Verzerrungsrate und eine geringe Heterogenität.

Kapitel vier beschreibt eine Machbarkeitsstudie in einem einfachblindierten randomisierten und kontrollierten Design mit einmaliger Intervention von Ganzkörpervibration mit stochastischer Resonanz (SR-GKV) bei No-Go und Slow-Go Probanden. Die Probanden in der Interventionsgruppe $(n=10)$ führten das SR-GKV auf dem Zeptor ${ }^{\circledR}$ Med durch. Hierfür standen die Probanden schulterbreit, mit leicht gebeugten Hüft-, Knie- und Fussgelenken und mit Schuhen auf zwei Fussplatten. Trainiert wurde fünf Mal eine Minute lang mit einer Intensität von $5 \mathrm{~Hz}$, Noise 4. Die Probanden der Kontrollgruppe $(n=10)$ sassen zur Placebo-Intervention zehn Minuten auf einem Stuhl. Erhoben wurden als primärer Zielparameter die „Kriterien für einen Erfolg“. Dies beinhalten die Rekrutierungsrate und die Adhärenz- und Ausfallrate. Als sekundärer Zielparameter wurden der Semitandemstand, Aufstehtest, Functional-Reach-Test, ExpandedTimed-Get-Up-and-Go-test, Einfachaufgabe, Doppelaufgabe, Hand- und Fussreaktionszeit ermittelt.

Die Machbarkeit dieser Studie konnte bestätigt werden. SR-GKV ist bei Go-Go und Slow-Go sicher anwendbar, es wurde gut vertragen und zeigte nutzbringende Effekte auf das Gleichgewicht.

Kapitel fünf beschreibt eine Machbarkeitsstudie mit stochastischer Ganzkörpervibration (SRGKV) über acht Wochen in einem einfachblindierten randomisierten und kontrollierten CrossOver-Design bei Go-Go und Slow-Go Probanden. 20 Probanden wurden zufällig der Gruppe A (vier Wochen SR-GKV mit $5 \mathrm{~Hz}$, Noise 4, anschl. 16 Tage Wash-Out-Periode, dann vier Wochen SR-GKV mit $1 \mathrm{~Hz}$, Noise 1) oder Gruppe B (vier Wochen SR-GKV mit $1 \mathrm{~Hz}$, Noise 1 , anschl. 16 Tage Wash-Out-Periode, dann vier Wochen SR-GKV mit $5 \mathrm{~Hz}$, Noise 4) zugeteilt. Die Probanden standen schulterbreit, mit leicht gebeugten Hüft-, Knie- und Fussgelenken und mit Schuhen auf zwei Fussplatten. Primärer Zielparameter waren die „Kriterien für den Erfolg“ (Rekrutierungsrate, die Adhärenz- und Ausfallrate) und die Sicherheit. Sekundäre Zielparameter waren der Semitandemstand, der Aufstehtest, der Functional-Reach-Test, der Expanded-TimedGet-Up-and-Go-test, single Task, dual Task und die Hand- und Fussreaktionszeit.

Diese Machbarkeitsstudie zeigte auf, dass das Untersuchungsprotokoll für zukünftige Studien verwendet werden kann. SR-GKV ist für Go-Go und Slow-Go Probanden sicher durchführbar. Diese Machbarkeitsstudie konnte genügend Probanden einschliessen (55\%) und zeigt mit 85\% eine akzeptable Adhärenzrate und eine Ausfallsrate von 15\%. Ein zwölfmaliges SR-GKV-Training mit $5 \mathrm{~Hz}$, Noise 4 scheint ausreichend starke Reize generieren zu können, um die Totalzeit im Expanded-Timed-Get-Up-and-Go zu verbessern.

Kapitel sechs schildert eine Machbarkeitsstudie bei No-Go Probanden mit stochastischer Ganzkörpervibration (SR-GKV) in einem doppelblindierten randomisierten und kontrollierten Cross-Over-Studiendesign. Von möglichen 24 Probanden konnten 9 Probanden eingeschlossen 
und zufällig derGruppe A (vier Wochen SR-GKV mit $6 \mathrm{~Hz}$, Noise 4, anschl. 16 Tage Wash-OutPeriode, dann vier Wochen SR-GKV mit $1 \mathrm{~Hz}$, Noise 1) oder Gruppe B (vier Wochen SR-GKV mit $1 \mathrm{~Hz}$, Noise 1, anschl. 16 Tage Wash-Out-Periode, dann vier Wochen SR-GKV mit $6 \mathrm{~Hz}$, Noise 4) zugeteilt werden. Die Probanden standen schulterbreit, mit leicht gebeugten Hüft-, Knie- und Fussgelenken mit Schuhen auf zwei Fussplatten. Zielparameter waren die „Kriterien für einen Erfolg" (Rekrutierungsrate, die Adhärenz- und Ausfallrate) und die Sicherheit. Primärer Zielparameter war der Expanded-Timed-Get-Up-and-Go-test und sekundärer Zielparameter der Short-Physical-Performance-Battery-Test, die Muskelkraft und der Aufstehtest (einmal durchgeführt).

Diese Machbarkeitsstudie illustrierte, dass das Untersuchungsprotokoll für zukünftige Studien angepasst werden muss. Die Ausfallsrate mit 56\% war sehr hoch und eine Adhärenzrate von 81.6\% mässig. Effekte zeigten sich in allen Variablen für SR-GKV Intervention mit $6 \mathrm{~Hz}$, Noise 4.

In Kapitel sieben, werden die Ergebnisse einer randomisierten einfachblindierten und kontrollierten Studie beschrieben. Diese Studie untersuchte die Effekte von stochastischer Ganzkörpervibration (SR-GKV) und einem Videospiel (VS) nach einem achtwöchigen Training auf die körperliche Leistungsfähigkeit und Muskelkraft bei pflegebedürftigen alten Menschen im Bereich der Langzeitpflege. Die Gruppeneinteilung wurde von einem unabhängigen Statistiker durchgeführt. 30 pflegebedürftige ältere Menschen wurden in eine Interventions- (IG, $n=16)$ oder Shamgruppe (SG, $n=14$ ) eingeteilt. Die IG absolvierte folgendes Programm: Acht Wochen lang, 3x/Woche, 5x 1 Minute Vibration mit 1 Minute Pause zwischen den Vibrationen. Das SRGKV begann mit einer Basisfrequenz von $3 \mathrm{~Hz}$, Noise 5 und wurde progressiv auf $6 \mathrm{~Hz}$, Noise 5 gesteigert. Ausgangsstellung (ASTE) war eine leichte Kniebeugung, aufrechter Oberkörper, möglich frei stehend und ohne Schuhe auf den zwei Fussplatten. Von Woche zu Woche wurde der Schwierigkeitsgrad der ASTE gesteigert. Vom Parallelstand über Schrittstellung zu dynamischen Kniebeugen bis maximal 90 Grad Knieflexion. Ab der fünften Woche kam das VS hinzu. Über einen Projektor wurden Pfeile auf eine Wand projiziert. Der Proband hatte die Aufgabe, im Takt der Musik auf die entsprechenden Pfeile der Tanzplatte zu treten - mal rechts, mal unten, mal links, usw.. Getanzt wurde zu fünf bis sechs Liedern von je anderthalb Minuten Dauer. Der Rhythmus wurde von Lied zu Lied schneller, von 32 auf 137 Schlägen pro Minute steigend. Die Probanden der Kotrollgruppe standen über acht Wochen auf dem SR-GKV, das mit $1 \mathrm{~Hz}$, Noise 1 vibrierte. Ab der 5. Woche wurde ein maschinell gesteuertes Trampolin eingesetzt, welches sinusförmige Schwingungen im Sekundentakt produziert. Um die Wirkung auf das Gleichgewicht auszuschalten, mussten sich die Probanden stetig am Geländer festhalten.

Als primärer Zielparameter diente der Short-Physical-Performance-Battery-Test. Sekundärer Zielparameter war die isometrische Maximalkraft, isometrische Schnellkraft der Oberschenkelstrecker und Oberschenkelbeuger. Zudem wurden Subwerte der isometrischen Maximalkraft bei 30, 50, 100 und 200ms als auch Subwerte der Schnellkraft im Bereich 0-30, 0-50, 0-100 und 100-200ms ermittelt. 
Mit dieser Studie konnte gezeigt werden, dass eine Kombination aus SR-GKV und VS die Outcomevariablen SPPB, Subwerte der Maximalkraft bei 30, 50, 100 und 200ms, die Schnellkraft und Subwerte der Schnellkraft zwischen 0-30, 0-50, 0-100 und 100-200ms signifikant verbesserte.

Kapitel acht präsentiert die Ergebnisse einer Validitätsstudie. Hierbei wurde die Validität des Beschleunigungsmessgeräts RehaWatch ${ }^{\circledR}$ System mit dem GAITRite ${ }^{\circledR}$ System während Einfach (EA)- und Doppelaufgaben (DA) verglichen. Erhoben wurden folgende Gangparameter: Standdauer rechts und links, Geschwindigkeit, Kadenz und Schrittlänge rechts und links. An dieser Studie nahmen 23 Probanden mit einem Durchschnittsalter von 90.90 ( \pm 8.4) Jahren teil. Die Probanden absolvierten vier verschiedene Messdurchgänge auf einer Gehstrecke von 13 Metern (normales Gehen - EA, normales Gehen - DA, schnelles Gehen - EA und schnelles Gehen - DA). Die Schlussfolgerung dieser Studie ist, dass nur die Werte der Kadenz valide sind und mit anderen Kadenzwerten verglichen werden dürfen. Des Weiteren hat diese Studie aufgezeigt, dass No-Go Probanden nicht mehr in der Lage sind, schneller Gehen zu können.

Kapitel neun diskutiert die wichtigsten Ergebnisse und die methodologischen Überlegungen dieser Thesis. Weiter werden Einschränkungen dieser Thesis kritisch beleuchtet. Daraus wurden Schlussfolgerungen gezogen, um Empfehlungen für zukünftige Forschungsarbeiten auszusprechen. Ausserdem werden Empfehlungen für die Praxis zur Implementierung des innovativen Trainingsprogramms für die No-Go Gruppe gegeben. 



\section{Acknowledgements}


I've had a few ups and downs on the way to completing my doctorate. During this time, l've had support and friendship from family, colleagues and friends that I would now like to acknowledge.

First of all, a special thanks to my Maastricht promoter Prof. Rob de Bie PhD, PT. He is a leader in the field of physiotherapeutic and rehabilitation settings. His ability to capture circumstances of the core issues of a research idea or research project, combined with his scientific experience makes him a great mentor for PhD students, guiding them through a PhD track. I thank Rob that I've had the possibility to experience that.

I would like to thank my local promoter Prof. Eling Douwe de Bruin PhD, PT, Movement Science. He has a profound scientific knowledge of training in seniors, has a large duty of care in ethics and scientific research and is an excellent strategist for research methods. Many thanks for the numerous conversations and your listening ear during my $\mathrm{PhD}$ track.

I must also mention Prof. Lorenz Radlinger, PhD, sport science, who was my supervisor at Bern University of Applied Sciences. He introduced me to the topic of whole-body vibration and enthused me about research. Throughout my PhD he encouraged me to investigate the field of gerontology research. Lorenz, thank you for spending time with me and your continuous interest in my research. You prepared the ground at the beginning of my PhD through numerous discussions and frequent feedback.

Prof. Dietmar Schmidtbleicher, PhD sport science, was my co-supervisor. He is a recognized expert in the field of strength training and whole-body vibration. Thank you for the support and countless advice and tips I have received during our meetings.

Ich bedanke mich bei der Senevita Residenz Multengut, Muri und ihren Bewohnern die an der Studie teilgenommen haben. Besonderer Dank gilt Frau Brigitte Stäldi, die mich bei der Durchführung der Studien in Muri sehr unterstützt hat. Weiter möchte ich mich bei den Mitarbeitern und Bewohnern im Schlössli Biel bedanken. Hier konnte ich meine dritte Studie durchführen. Frau Monika Eichelberger und Annegrette Birle waren von Anfang an für diese Studie offen. Danke Annegrette, dass du während dieser Phase immer als Ansprechperson der Teilnehmer da warst.

My employer, the Discipline of Physiotherapy in the Department of Health, who gave me the necessary support and breathing space in parallel to the ongoing working process at BUAS to work on my PhD research project. A special thank-you to Prof. Heiner Baur PhD, sport science, for his continual support during the clinical study in Biel, Switzerland. Thank you Prof. Dr. Jan Taeymans for the support during my PhD track. 
I would also like to thanks my colleagues Drs. Patric Eichelberger, Drs. Stefan Schmid, Yvonne Brülhart, MSc, PT, Prof. Ross Bennie, BSc and Kaspar Herren, MSc, PT for their support during my PhD track.

Special thanks to Roger Hilfiker cand. PhD, PT. Your human nature, paired with clinical knowledge and enormous scientific understanding let me see things from a different point of view.

There are a number of friends who have supported me on the way to my PhD. Many thanks to Helga; you've supported and encouraged me from the beginning. I am proud that you are one of my paranymphs - thank you very much, Helga. No effort appeared to be hard. I also want to express my thanks to Michael. You gave me important inputs throughout my PhD track and helped me find solutions, particularly during the writing of my thesis.

A special thank goes to my friend and paranymphs Klaus Acker. Auch möchte ich mich bei Mariette und Herbert bedanken, die während meinem PhD-Prozess als ruhender Pol meine Belastungen oder auch Sorgen vergessen liessen.

Meinen Eltern und Schwerster möchte ich danken, die es mir ermöglichten als Voraussetzung für den PhD, den Bachelor- und Masterstudiengang zu absolvieren.

I would like to thank the reading committee members of may PhD Thesis and corona for their interest in my work and their time on reading it: Prof. dr. H. Savelberg (chairman), Prof. dr. J.M.G.A. Schols, Dr. K. Meijer, PD dr. med S. Bachmann and PD dr. med T. Hinrichs. 

About the author 


\section{Curriculum Vitae}

Slavko Rogan was born in Wehr/Baden, Germany. In 1992 he completed training as a masseur and medical spa therapist at the Massageschule in Bad Säckingen. In 2000 he qualified as a physiotherapist at the Physiotherapie Schule (PHYTHERAS) in Bad Krozingen, Germany. After basic education in Germany he studied physiotherapy as an external student, graduating in 2003 with an Bc and 2007 with an MSc.

Slavko accumulated 17 years of clinical experience in sport rehabilitation and musculoskeletal conditions before his 2007 appointment as lecturer and researcher in the Department of Health at Bern University of Applied Sciences.

In 2010, he started his PhD track at CAPHRI (Promoter: Prof. dr. Rob A. de Bie) and the Institute of Human Movement Sciences and Sport, Department of Health Sciences and Technology, Swiss Federal Institute of Technology Zurich (Co-promoter: PD Dr. Eling D. de Bruin).

Concurrently to his $\mathrm{PhD}$, Slavko has been studying for an MA in Further Adult Education at the University of Kaiserslautern, which he graduated in March 2016. Additionally, in 2014 he completed a five-year apprenticeship in Osteopathy and extended his academic study to an MSc in Osteopathy.

Slavko lives in Kaiseraugst, near Basel in Switzerland. 


\section{Publication}

\section{Books / Book Chapter}

- Rogan S (2015) Begleitendes Selbststudium in der Hochschulausbildung Physiotherapie, eBook, GRIN Verlag

- Wirz M, Köhler B, Marks D, Kool J, Sattelmeier M, Oesch O, Hilfiker R, Rogan S \& Schädler S (2014) Lehrbuch Assessments in der Rehabilitation, Huber Verlag.

\section{Experten-Interview}

- Anja Stamm Erfolgreiches Doppel. meine physiopraxis. 2014; 03

\section{Protocol systematic Review}

- Buss B, Lujickx E, Brandt S \& Rogan S Portfolio: a review of literature and implications for physiotherapy education. PROSPERO (International prospective register of systematic review)

- Rogan S, Taeymans, J, Radlinger L, Näpflin S B, Ruppen S, Brülhart Y \& Hilfiker R Effects of whole-body vibration on postural control in elderly: update of a systematic review and metaanalysis. PROSPERO (International prospective register of systematic review)

- Jaspers T, Taeymans $J$ \& Rogan $S$ What are the effects of continuous passive motion on range of motion, swelling and pain after surgery in ACL-reconstruction patients? PROSPERO (International prospective register of systematic review)

- Rogan S, Luijckx E, Dähler J, Reuteler S \& Taeymans J Treatment of patellofemoral pain syndrome: systematic literature review PROSPERO (International prospective register of systematic review)

- Rogan S, Gebruers N, Luginbühl H, Luijckx E, Aebi M, Mahnig S, Schär V, Wermuth N \& Taeymans J Therapy modalities to reduce secondary lymphedema in female breast cancer patients: a systematic review and meta-analysis \& PROSPERO (International prospective register of systematic review)

- Rogan S, de Bruin ED, Brülhart Y \& Hilfiker R Strength effects after whole-body vibration in patients with osteoarthritis - a systematic review and meta-analysis PROSPERO (International prospective register of systematic review)

- Rogan S, de Bruin E, Radlinger L, Jöhr C, Nyffenegger C, Stuck N-J, de Bie R \& Hilfiker R Strength effects after whole-body vibration in elderly population - a systematic review and meta-analysis \& PROSPERO (International prospective register of systematic review)

- Rogan S, Hilfiker R, Schenk A, Vogler A \& Taeymans J Effects of whole-body vibration with stochastic resonance on postural control - a systematic review

- Rogan S, Herren K, Hilfiker R, Radlinger L, R \& de Bruin ED Effects of whole-body vibration on postural control: a systematic review and meta-analysis 


\section{Per-reviewed Journal Publications}

- Rogan S, de Bie RA \& de Bruin ED Agreement between a sensor-based feet mounted wearable and a pressure sensitive gait analysis system in long term care dwelling frail older adults. Z Gerontol Geriat. 2016. [Epub ahead of print]

- Rogan S, Taeymans J, Luginbuehl H, Aebi M, Mahnig S \& Gebruers N Therapy modalities to reduce lymphedema in female breasts cancer patients: a systematic review and metaanalysis. Breast Cancer Research and Treatment. 2016

- Rogan S, Brülhart Y, Ledermann T, Schmutz N \& Lujickx E Effectiveness of muscle stretching during interferential current in soccer players - a pilot study. Journal of Physical Education and Sport (JPES). 2016. 16(2):465-470

- Jaggi J, Kneubühler S \& Rogan S [Influence of ankle brace on the prevalence of ankle inversion injuries in the Swiss Volleyball National League A]. Sportverletzung-Sportschaden. 2016. 30: 101-105

- Rogan S, Radlinger L, Baur H, Schmidtbleicher D, de Bie RA \& de Bruin ED Sensory-motor training targeting motor dysfunction and muscle weakness in long-term elderly combined with motivational strategies: a single blind randomized controlled study. European Review of Aging and Physical Activity. 2016. 13: 4

- Rogan S., Taeymans J, Schürmann S, Wörn L-M, Clarys P, Clijsen R [Segmental skin blood reaction during and after thoracic spine stimulation techniques - a single case study for building a neurophysiological hypothesis]. physioscience. 2016. accepted

- Obrist S, Rogan S \& Hilfiker R Development and evaluation of an online fall risk questionnaire for non-frail community dwelling elderly persons. A pilot study. Current Gerontology and Geriatrics Research. 2016; 1520932

- Rogan S \& Radlinger L. From No-Go to Go-Go - Future training procedures for elderly. Journal of Gerontology \& Geriatrics Research. 2016; 5.1

- Rogan S, de Bruin ED, Radlinger L, Jöhr C, Wyss C, Stuck N-J, Brülhart Y, de Bie R A \& Hilfiker $R$ Effects of whole-body vibration on proxies of muscle strength in old adults: a systematic review and meta-analysis on the role of physical capacity level. European Review of Aging and Physical Activity. 2015; 12:12

- Rogan S, Radlinger L, Schmidtbleicher D, de Bie R \& de Bruin ED Preliminary inconclusive results of a randomised double blinded cross-over pilot trial in long-term-care dwelling elderly assessing the feasibility of stochastic resonance whole-body vibration. European Review of Aging and Physical Activity. 2015; 12:5

- Jäggi U, Joray C, Brülhart Y, Luijckx E \& Rogan S Verletzungen in den Kampfsportarten Judo, Taekwondo und Ringen - eine systematische Übersichtsarbeit. Sportverletzung-Sportschaden. 2015; 29:219-225

- Jaggi J, Kneubühler S \& Rogan S [Influence of ankle brace on the prevalence of ankle inversion injuries in the Swiss Volleyball National League A]. Sportverletzung-Sportschaden. 2015 Nov [Epub ahead of print]

- Ferraro M, Stalder S, Baur H, Radlinger L \& Rogan S Evaluation of a foot switch system for the maximal instep kick in soccer - results from a single-case study. Journal of Physical Education 
and Sports (JPES). 2015; 15(1): 57-63.

- Rogan S, Radlinger L, Imhasly C, Kneubühler A \& Hilfiker R Validity study of a jump mat compared to the reference standard force plate. Asian Journal Sports Medicine. 2015; 6(4):e25561

- Agner S, Bernet J, Brülhart Y, Radlinger L \& Rogan S Spatio-temporal gait parameters during dual task in need of care elderly and young adults - a cross sectional study. Z Gerontol Geriat. 2015; 48(8):740-746 (Impact Factor: 1.023)

- Rogan S, Radlinger L, Hilfiker R, Schmidtbleicher D, de Bie R \& de Bruin ED Feasibility and effects applying stochastic resonance whole-body vibration on untrained elderly: A randomized crossover pilot study. BMC Geriatr. 2015; 15: 25 (Impact Factor: 2.0)

- Rogan S Comparison of two kinds of endurance training programs on the effects of the ability to recover in amateur soccer players. Asian J Sports Medicine. 2015;6 (2):e22585. DOI: 10.5812/asjsm.6(2)2015.22585

- Rogan S, Riesen J \& Taeymans J [Core muscle chains activation during core exercises determined by EMG - systematic review]. Praxis. 2014. 103 (21): 1263-1270. DOI 10.1024/16618157/a001803. ( $\mathrm{H}$ index: 11)

- Rogan S, Wüst D, Ringgenberg M, Sager R \& Schmidtbleicher D Comparison of endurance performance and stretch-shortening cycle between two male amateur soccer teams - a crosssectional pilot study. Asian J Sports Medicine. 2014.

- Kessler J, Radlinger L, Baur H \& Rogan S Effect of stochastic resonance whole-body vibration on functional performance in the frail elderly: a pilot study. Archives of Gerontology and Geriatrics. 2014; 59 (2): 305-311 (Impact Factor: 1.704)

- Rogan S, Hilfiker R, Schenk A, Vogler A \& Taeymans J Effects of whole-body vibration with stochastic resonance on balance in persons with balance disability and falls history - a systematic review. Res Sports Med. 2014; 22(3):294-313 (Impact Factor: 1.143)

- Calendo, L-R, Taeymans J \& Rogan S [Does muscle activation during whole-body vibration induce bone density improvement in postmenopausal women? - a systematic review]. Sportverletzung-Sportschaden. 2014; [Epub ahead of print] (Impact Factor: 0.61)

- Rogan S, Schmidtbleicher D \& Radlinger L Immediate effects after stochastic resonance whole-body vibration on physical performance on frail elderly for skilling up training: a blind cross-over randomised pilot study. Aging Clin Exp Res. 2014; [Epub ahead of print] (Impact Factor: 1.006)

- Rogan S, Baur H, Sargent A, Schori M \& Taeymans J [Feasibility of a balance training on Airexmats in healthy, moderately sportive women in old age - a pilot study]. Z Gerontol Geriat. 2015; 48(2): 135-141 (Impact Factor: 1.023)

- Rogan S, Blasimann A, Nyffenegger D, Zimmerli N \& Radlinger L [The relevance of core muscle in ice hockey players: a feasibilty study]. Sportverletzung-Sportschaden. 2013; 27(4):212-218 (Impact Factor: 0.61) 
- Rogan S Klassifikation in der Physiotherapie erklärt anhand von Rückenschmerz. manuelletherapie. 2013;17(5):201-205

- Rogan S, Radlinger L, Portner-Burkhalter C, Sommer A \& Schmidtbleicher D Feasibility study evaluating four weeks stochastic resonance whole-body vibration training with healthy female student. International Journal of Kinesiology \& Sport Science. 2013;1(2):1-9

- Rogan S, Taeymans J, Hirschmüller A, Niemayer P \& Baur H Wirkung von passiven Motorbewegungsschienen nach Knorpelregenerativen Eingriffen - eine systematische Literaturübersicht. Zeitschrift für Orthopädie und Unfallchiriurgie. 2013; 151:468-474 (Impact Factor: 0.522)

- Rogan S, Wüst D, Schwitter T \& Schmidtbleicher D Static stretching of the hamstring muscle for injury prevention in football codes: a systematic review. Asian J Sports Medicine. 2013;4(1):1-9

- Rogan S \& Hilfiker R Muskelkraftsteigerung durch Ganzkörpervibration. SportverletzungSportschaden. 2012;24(4):185-187 (Impact Factor: 0.61)

- Rogan S, Blasimann A, Steiger M, Torre A \& Radlinger $L$ [Acute effects of fast dynamic stretching on rate of force development in ice hockey players: a pilot study]. SportverletzungSportschaden. 2012;26(4):207-211 (Impact Factor: 0.61)

- Rogan S, Hilfiker S, Schmid S \& Radlinger L Stochastic resonance whole-body vibration training for chair rising performance on untrained elderly: a pilot study. Archives of Gerontology and Geriatrics. 2012;55(2):468-473 (Impact Factor: 1.704)

- Rogan S, Radlinger L, Schmid S, Herren K, Hilfiker R \& de Bruin ED. Skilling up for training: a feasibility study investigating acute effects of stochastic resonance whole-body vibration on postural control of older adults. Ageing Research. 2012;3(1):e5

- Rogan S, Hilfiker R, Herren K, Radlinger \& de Bruin ED. Effects of whole-body vibration on postural control in elderly: a systematic review and meta-analysis. BMC Geriatr. 2011 Nov 3;11:72 (Impact Factor: 2.34)

- Rogan S, Hilfiker R, Clarys P, Clijsen R \& Taeymans J Position-specific and Team-ranking related morphological characteristics in German Amateur soccer players - a descriptive study. IJASS. 2011;23(1):168 - 182

\section{Non Per-Reviewed Journal Publications}

- Wüst D \& Rogan S Physiotherapie im Profifussball. med \& move 2016. accepted

- Wolf M \& Rogan S Sichere Mobilität für Senioren. Heime und Spitäler. 2015.

- Rogan S \& Radlinger L Sturzprävention: mehr Sicherheit. med \& move. 2015; 32-34

- Rogan S Erfolgreiches Bewegungstraining für gebrechliche Betagte. Heime und Spitäler. 2014; 3: 24-25

- Rogan S \& Hilfiker R Muskelkraftsteigerung durch Ganzkörpervibration. Physiopraxis. 2012; 10: $40-43$

- Rogan S Muskelkraftmessung Alternativen zur manuellen Muskelfunktionsmessung- Einsatz in der Praxis. pt_Zeitschrift für Physiotherapeuten. 2012; 64(11): 46-49

- Rogan S Muskelkraftmessung Alternativen zur manuellen Muskelfunktionsmessung - 
Grundlagen. pt_Zeitschrift für Physiotherapeuten. 2012; 64(10):48-51

- Herren K, Rogan S \& Radlinger L Ganzkörpervibration als Krafttrainingsmethode. Physioactive. 2012; 5:23-29

- Rogan S, Pichierri G \& de Bruin E Denk Sport-Dual Taskig Training mindert Sturzrisiko. Ergopraxis. 2012;3:18-21

- Rogan S, Pichierri G \& de Bruin E Denk Sport-Dual Taskig Training mindert Sturzrisiko. Physiopraxis. 2011;10:34-37

- Rogan S \& Leitner M Bern-Amsterdam retour: Am Weltkongress der Physiotherapie 2011. Frequenz. 2011;28

- Rogan S \& Stäldi B verhelfen Vibrationen zu einem sicheren Stand? terz. 2010;16-17

- Herren K, Rogan S, Hilfiker R \& Radlinger L Vibrationen mit therapeutischen interessanten Effekten. Physioactive. 2009;5:39-44

- Rogan S Nach dem Training ist vor dem Training. Physiopraxis. 2008;6:24-28 
